\title{
A colimit construction for groupoids
}

\author{
Dissertation
}

zur Erlangung des mathematisch-naturwissenschaftlichen Doktorgrades

„Doctor rerum naturalium“ (Dr.rer.nat.)

der Georg-August-Universität Göttingen

im Promotionsprogramm der PhD School of Mathematical Science (SMS) der Georg-August University School of Science (GAUSS)

vorgelegt von

Suliman Albandik

aus Latakia, Syrien

Göttingen 2015 


\title{
Betreungsausschuss:
}

\author{
Betreuer: Prof. Dr. Ralf Meyer \\ Mathematisches Institut, Georg-August-Universität Göttingen \\ Betreuer: Prof. Dr. Ingo Witt \\ Mathematisches Institut, Georg-August-Universität Göttingen \\ Betreuer: Prof. Dr. Dorothea Bahns \\ Mathematisches Institut, Georg-August-Universität Göttingen
}

Mitglieder der Prüfungskommission:

\author{
Referent: Prof. Dr. Ralf Meyer \\ Mathematisches Institut, Georg-August-Universität Göttingen \\ Koreferent: Prof. Dr. Alcides Buss \\ Department of Mathematics, Federal University of Santa Catarina, Brazil
}

Prof. Dr. Anja Fischer

Institut für Numerische und Angewandte Mathematik, Georg-August-Universität Göttingen

Prof. Dr. Ingo Witt

Mathematisches Institut, Georg-August-Universität Göttingen

Prof. Dr. Karl Henning Rehren

Institut für Theoretische Physik, Georg-August-Universität Göttingen

Prof. Dr. Jörg Brüdern

Mathematisches Institut, Georg-August-Universität Göttingen 
Tag der mündlichen Prüfung: 10.08.2015 
To my dearest: Hayder, Jousef, Danial, Lamma and my beloved family 


\begin{abstract}
We consider Ore monoid actions in a certain bicategory of étale groupoids $\mathcal{G}_{\text {prop }}$. Examples of such actions include self-similar groups, higher rank graphs and actions of Ore monoids on spaces by topological correspondences. We prove that every Ore monoid action in $\mathcal{G}_{\text {prop }}$ has a colimit. We construct a functor from $\mathcal{G}_{\text {prop }}$ to the bicategory of $\mathrm{C}^{*}$-correspondences $\mathfrak{C o r r}$. We prove that this functor preserves colimits of Ore monoid actions. We write the colimit of an Ore monoid action concretely, and in doing so provide a groupoid model for the Cuntz-Pimsner algebra of the product system associated with the action. In the second part of this thesis, we study colimit equivalence in the bicategories $\mathfrak{C o r r}$ and $\mathfrak{G r}$. We show that under certain assumptions on a diagram, cofinal subdiagrams have equivalent colimits. This generalises the notions of shift equivalences of graphs and $\mathrm{C}^{*}$-correspondences.
\end{abstract}




\section{Acknowledgments}

First and foremost, I am using this opportunity to express my sincere gratitude to my main supervisor, Prof. Dr. Ralf Meyer for his guidance, patience and encouragement.

I am grateful to Prof. Dr. Alcides Buss who has agreed to be a second referee for this thesis.

I am also thankful to the people at the Mathematisches Institut in Göttingen for providing the great environment for research.

Finally, I especially thank my parents, my brothers, my sister and my close friends for their invaluable support. 



\section{Contents}

1 Introduction 1

1.1 Motivation . . . . . . . . . . . . . . . . . . 1

1.2 The bicategory of groupoid correspondences . . . . . . . . . . . 3

1.3 Colimit equivalent dynamical systems . . . . . . . . . . . 5

1.4 Overview of the thesis . . . . . . . . . . . . . . 6

2 Groupoid bicategories and the bicategory of $\mathrm{C}^{*}$-correspondences 9

2.1 Bicategories . . . . . . . . . . . . . . . . . . . 9 9

2.2 Groupoid correspondences . . . . . . . . . . . . . . . . . 13

2.2.1 Groupoid correspondences . . . . . . . . . . . . . . 16

2.2 .2 Examples . . . . . . . . . . . . . . . . . 17

2.3 The composition of groupoid correspondences . . . . . . . . . . 20

2.4 Bicategories of groupoid correspondences . . . . . . . . . . . 22

2.5 The functor to $\mathfrak{C o r r} \ldots \ldots \ldots \ldots \ldots \ldots \ldots$

2.6 Diagrams and colimits . . . . . . . . . . . . . . . 30

2.6.1 Diagrams of groupoid correspondences . . . . . . . . . 30

2.6.2 Diagrams of $\mathrm{C}^{*}$-correspondences . . . . . . . . . . 33

2.6.3 Examples ...................... 35

3 Actions of Ore monoids $\quad 37$

3.1 Ore monoids . . . . . . . . . . . . . . . . . 37

3.2 Actions of Ore monoids by tight correspondences . . . . . . . . . 39

3.2.1 The construction of the colimit groupoid . . . . . . . . . 41

3.2.2 Properties of the colimit groupoid . . . . . . . . . . 48

3.2.3 Product systems associated with tight Ore monoid actions . 51

3.3 Actions of Ore monoids by proper correspondences . . . . . . . . . 56

3.3.1 Tightening a proper Ore monoid action . . . . . . . 56

3.3.2 The product systems . . . . . . . . . . . . . . . 64

3.4 Examples . . . . . . . . . . . . . . . . . . . . 69

3.4.1 Actions by local homeomorphisms . . . . . . . . . . 69

3.4 .2 Self-similar group actions . . . . . . . . . . . 71

4 Colimit equivalent diagrams $\quad \mathbf{7 7}$

4.1 Colimit equivalent diagrams in general bicategories . . . . . . . . 77 


\section{Contents}

4.2 Applications . . . . . . . . . . . . . . . . . 85

4.2.1 Shift equivalence of $\mathrm{C}^{*}$-correspondences $\ldots \ldots \ldots . . .85$ 


\section{Introduction}

\subsection{Motivation}

If a locally compact group $G$ acts on a locally compact Hausdorff space $X$ by homeomorphisms, one can form the full crossed product $G \ltimes \mathrm{C}_{0}(X)$ of $\mathrm{C}_{0}(X)$ by $G$. We think of $G \ltimes \mathrm{C}_{0}(X)$ as a skew tensor product of the full group $\mathrm{C}^{*}$-algebra $\mathrm{C}^{*}(G)$ with $\mathrm{C}_{0}(X)$. A celebrated result of Rieffel [42] shows that $\mathrm{C}_{0}(X / G)$ is Morita equivalent to $G \ltimes \mathrm{C}_{0}(X)$ when the group action is free and proper. This suggests to call the crossed product $G \ltimes \mathrm{C}_{0}(X)$ a noncommutative quotient, see [12,21]. So from the point of view of noncommutative geometry, the crossed product $G \ltimes \mathrm{C}_{0}(X)$ replaces the quotient space $X / G$. If the group $G$ acts freely and properly on $X$, then $X / G$ is Hausdorff. But what happens for non-Hausdorff quotients? In such cases the quotient space is not suitable to encode the group action, as we will illustrate in the following example. Let $\theta \in \mathbb{R}$ be a fixed real number. We consider the rotation action of $\mathbb{Z}$ on the unit circle $\mathbb{T}$ given by

$$
(n, z) \mapsto e^{2 \pi i n \theta} z
$$

If $\theta$ is a rational number then the quotient space $\mathbb{T} / \mathbb{Z}$ is a circle. Hence

$$
{ }^{\prime \prime} \mathrm{C}(\mathbb{T} / \mathbb{Z})^{\prime \prime}:=\{f \in \mathrm{C}(\mathbb{T}) \mid f(n z)=f(z) \text { for all } n \in \mathbb{Z}, z \in \mathbb{T}\} \cong \mathrm{C}(\mathbb{T}) .
$$

If $\theta$ is irrational then the action is free, but not proper; the quotient space $\mathbb{T} / \mathbb{Z}$ has only two open sets and therefore ${ }^{\prime \prime} \mathrm{C}(\mathbb{T} / \mathbb{Z})^{\prime \prime} \cong \mathbb{C}$. In both cases, the crossed product $\mathbb{Z} \ltimes \mathrm{C}(\mathbb{T})$ is isomorphic to the noncommutative torus $A_{\theta}$.

There is, however, a natural groupoid $G \ltimes X$ associated with an action of $G$ on $X$ which may replace $X / G$. The object space of $G \ltimes X$ is $X$ and the arrow space is $G \times X$. The source and range maps are

$$
r(g, x)=g(x), \quad s(g, x)=x
$$

and the multiplication map is

$$
(g, x)(h, y)=(g h, y)
$$

if $h(y)=x$. The inverses are defined by $(g, x)^{-1}=\left(g^{-1}, g(x)\right)$. While the groupoid $G \ltimes X$ remembers the isotropy groups, the quotient space $X / G$ totally 


\section{Introduction}

forgets this information. Moreover, $\mathrm{C}^{*}(G \ltimes X)$ is isomorphic to $G \ltimes \mathrm{C}_{0}(X)$. This makes $G \ltimes X$ the right object to study from the perspective of noncommutative geometry. Another reason to use a groupoid as a middle step when associating a $\mathrm{C}^{*}$-algebra to given topological data is the well developed theory of groupoid $\mathrm{C}^{*}$-algebras. The existence of a groupoid model for a $\mathrm{C}^{*}$-algebra allows to use the general theory of groupoid algebras to study its basic structural properties, such as simplicity, nuclearity or pure infiniteness.

In the previous example, we started with an action of a group on a space. This is an instance of a reversible dynamical system. A prototype for a non-reversible dynamical system can be obtained from a bouquet of $n$ loops $X$ over a vertex $\{\star\}$ for a fixed natural number $n \in \mathbb{N}$. This corresponds to an action of $\mathbb{N}$ on the set $\{\star\}$ by the graph $X:\{\star\} \rightarrow\{\star\}$, where a natural number $k \in \mathbb{N}$ acts on $\{\star\}$ by the product space $X^{k}$ realised as a bouquet of $n^{k}$ loops over $\{\star\}$. The graph $X:\{\star\} \rightarrow\{\star\}$ gives a $\mathrm{C}^{*}$-correspondence $\mathbb{C}^{n}: \mathbb{C} \rightarrow \mathbb{C}$, see Section 2.5. The Cuntz-algebra $\mathcal{O}_{n}$ is the right $\mathrm{C}^{*}$-algebra to associate with the correspondence $\mathbb{C}^{n}: \mathbb{C} \rightarrow \mathbb{C}$. It is generated by isometries $S_{1}, \ldots, S_{n}$ such that $\sum_{i=1}^{n} S_{i} S_{i}^{*}=1$. There is also a groupoid $\mathcal{G}_{X}$, the Cuntz groupoid, associated with the graph $X$ with $\mathrm{C}^{*}\left(\mathcal{G}_{X}\right) \cong \mathcal{O}_{n}$, see $[40$, p. 140].

The construction of the Cuntz algebra has been generalised to what is now called Cuntz-Pimsner algebras, which were introduced by Pimsner in [39]. Pimsner constructs a $\mathrm{C}^{*}$-algebra from a $\mathrm{C}^{*}$-correspondence over a $\mathrm{C}^{*}$-algebra. CuntzPimsner algebras have been receiving continuous interest as generalised crossed products associated to possibly non-invertible, singly generated $\mathrm{C}^{*}$-dynamics. An action by a $\mathrm{C}^{*}$-correspondence is equivalent to an action of the monoid $\mathbb{N}$. Actions of a general monoid may be defined by putting together several $\mathrm{C}^{*}$-correspondences in a suitable way. The correct definition is that of an (essential) product system over the monoid; product systems were introduced by Fowler [17], who was inspired by earlier work of Arveson [3]. We have shown in [2] that the Cuntz-Pimsner algebra of a product system is the right analogue of the crossed product for monoid actions by proper $\mathrm{C}^{*}$-correspondences.

An important source of product systems is monoid actions by topological correspondences. A topological correspondence is a quadruple $(E, V, r, s)$, where $E$ and $V$ are locally compact spaces, $r: E \rightarrow V$ is a continuous map and $s: E \rightarrow V$ is a local homeomorphism. An action of an Ore monoid $P$ on a space $X$ by topological correspondences gives rise to a product system in the bicategory of $\mathrm{C}^{*}$-correspondences. One of our main results in [1] is the construction of a groupoid model for the Cuntz-Pimsner algebra associated to such a product system. This generalises constructions for graph algebras [23] and higher-rank topological graph algebras [46]. As we will be working simultaneously on two tracks-on the groupoid level and on the $\mathrm{C}^{*}$-algebraic level-it is helpful to keep in mind the following diagram regarding $\mathrm{C}^{*}$-algebras associated with Ore monoid actions by topological 
correspondences.

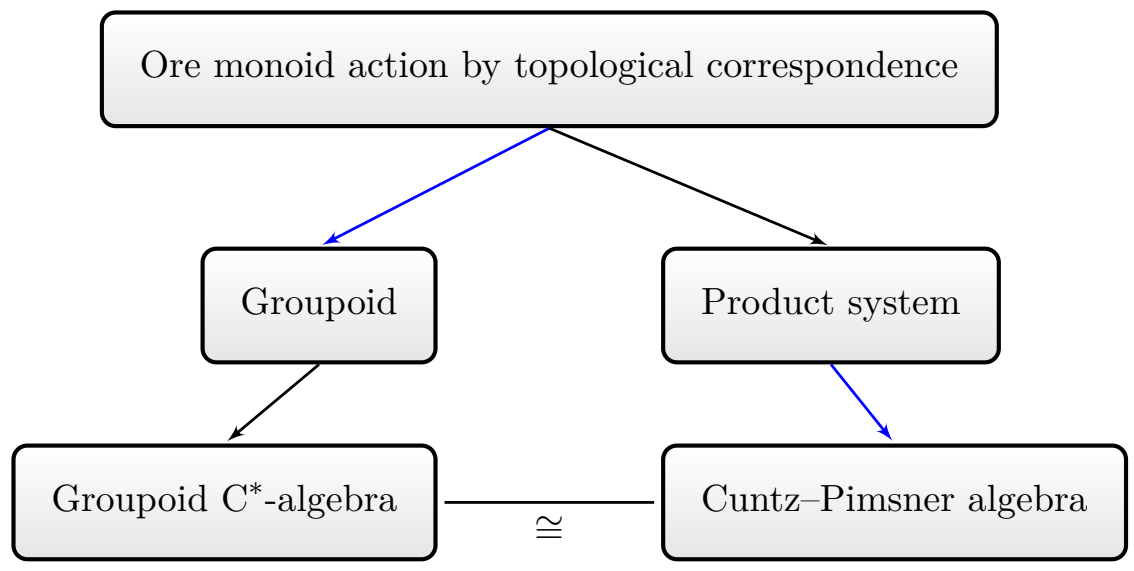

In this work we are interested in generalizing this result. But before explaining this let us analyze the previous examples further. Cuntz-Pimsner algebras of product systems satisfy certain universal properties, which make them colimits of the associated diagrams in a certain bicategory of $\mathrm{C}^{*}$-algebras, see [2]. Colimits are canonical constructions to build new objects from simpler ones. For a group action $(G, X)$, the crossed product $G \ltimes \mathrm{C}_{0}(X)$ is also a colimit of the associated diagram $\left(G, \mathrm{C}_{0}(X)\right)$. If we view the action $(G, X)$ as a diagram of topological spaces, then its colimit is the quotient space $X / G$. In an appropriate bicategory of groupoids its colimit is the transformation groupoid $G \ltimes X$.

There were several attempts to find an appropriate categorical setting to study dynamical systems. In [24], a category $\mathcal{M}$ of discrete higher-rank graphs has been introduced. The authors in [24] also introduce a functor from $\mathcal{M}$ to the category of $\mathrm{C}^{*}$-algebras and isomorphism classes of Hilbert bimodules as arrows. This makes $\mathcal{M}$ attractive as a possible setup for defining generalised dynamical systems. Moreover, if $\Lambda$ is a discrete higher-rank graph and $X: \Lambda \rightarrow \Lambda$ is an endomorphism in $\mathcal{M}$, then a new higher-rank graph $\Lambda \times_{X} \mathbb{N}$ (called the skew graph) may be constructed. The $\mathrm{C}^{*}$-algebra of the graph $\Lambda \times_{X} \mathbb{N}$ is naturally isomorphic to the Cuntz-Pimsner algebra $\mathcal{O}_{\mathrm{C}^{*}(X)}$, see [24, Theorem 6.8]. Nevertheless, despite their combinatorial structure and their nice $\mathrm{C}^{*}$-algebras, discrete higher-rank graphs are not well-suited as a common ground to study dynamical systems. Many examples are left untreated and an extra step is usually needed to link this category to a suitable category of groupoids.

\subsection{The bicategory of groupoid correspondences}

In 1967, Bénabou introduced bicategories in [6]. In a bicategory, we replace the sets of arrows between objects by categories of arrows. The composition of arrows 


\section{Introduction}

becomes a bifunctor. We demand that the associativity and unitality hold only up to isomorphisms of functors.

We study colimits mainly in bicategories. The reason for this is best explained by the following example. Let $A$ be a $\mathrm{C}^{*}$-algebra and let $G$ be a group acting on $A$ by isomorphisms $\left(\alpha_{g}\right)_{g \in G}$. The colimit of this action in the usual category of $\mathrm{C}^{*}$-algebras, which consists of $\mathrm{C}^{*}$-algebras as objects and *-homomorphisms as arrows, is not an interesting object. It is the largest quotient of $A$ such that all isomorphisms $\alpha_{g}$ are trivial on this quotient. On the contrary, the colimit of this action in the bicategory of $\mathrm{C}^{*}$-correspondences is the crossed product $G \ltimes A$.

What are diagrams in categories and their colimits? Let $\mathcal{C}$ and $\mathcal{D}$ be categories. A diagram in $\mathcal{D}$ of shape $\mathcal{C}$ is a functor $\mathcal{C} \rightarrow \mathcal{D}$. Such diagrams are again the objects of a category $\mathcal{D}^{\mathcal{C}}$, with natural transformations between functors as arrows. Any object $x$ of $\mathcal{D}$ gives rise to a "constant" diagram const ${ }_{x}: \mathcal{C} \rightarrow \mathcal{D}$ of shape $\mathcal{C}$. The colimit colim $F$ of a diagram $F: \mathcal{C} \rightarrow \mathcal{D}$ is an object of $\mathcal{D}$ with the following universal property: there is a natural bijection between arrows colim $F \rightarrow x$ in $\mathcal{D}$ and transformations $F \Rightarrow$ const $_{x}$ for all objects $x$ of $\mathcal{D}$. In brief,

$$
\mathcal{D}(\operatorname{colim} F, x) \cong \mathcal{D}^{\mathcal{C}}\left(F, \text { const }_{x}\right) .
$$

Let $\mathcal{C}$ and $\mathcal{D}$ be bicategories. A diagram in $\mathcal{D}$ of shape $\mathcal{C}$ is a functor $\mathcal{C} \rightarrow \mathcal{D}$. The functors $\mathcal{C} \rightarrow \mathcal{D}$ are the objects of a bicategory $\mathcal{D}^{\mathcal{C}}$; arrows and 2-arrows in this bicategory are called transformations between functors and modifications between transformations, see [25].

Thus $\mathcal{D}^{\mathcal{C}}\left(F_{1}, F_{2}\right)$ for two diagrams $F_{1}$ and $F_{2}$ is a category, not just a set. Similarly, for two objects $x_{1}$ and $x_{2}$ of $\mathcal{D}$, there is a category $\mathcal{D}\left(x_{1}, x_{2}\right)$ of arrows $x_{1} \rightarrow x_{2}$ and 2-arrows between them. Once again, there is a constant diagram const $x_{x}$ of shape $\mathcal{C}$ for any object $x$ of $\mathcal{D}$. The bicategorical colimit is defined by the same condition (1.1) after interpreting $\cong$ as a natural equivalence of categories. The colimit colim $F$ of some diagram $\mathcal{D}$ is unique up to equivalence if it exists.

Our main interest lies in the realm of groupoids and $\mathrm{C}^{*}$-algebras. In the following we introduce bicategories $\mathfrak{C o r r}$ with $\mathrm{C}^{*}$-algebras and $\mathfrak{G r}$ with groupoids as objects. First, let $A, B$ be $\mathrm{C}^{*}$-algebras. A correspondence from $A$ to $B$ is a right Hilbert $B$-module $\mathcal{E}$ with a non-degenerate ${ }^{*}$-homomorphism $\varphi$ from $A$ to the $\mathrm{C}^{*}$-algebra of adjointable operators on $\mathcal{E}$. The correspondence $\mathcal{E}$ is called proper if the image of $\varphi$ is contained in the $\mathrm{C}^{*}$-algebras of compact operators. An isomorphism between two such correspondences is a unitary operator which intertwines the left $A$-actions. Let $\mathfrak{C o r r}$ be the bicategory consisting of $\mathrm{C}^{*}$-algebras as objects, correspondences as arrows and isomorphisms of correspondences as 2 -arrows. Let $\mathcal{C}$ be a category. A diagram of shape $\mathcal{C}$ in $\mathfrak{C o r r}$ is a functor $\mathcal{C} \rightarrow$ Corr. Such diagrams are the objects of a bicategory $\mathfrak{C o r r}^{\mathcal{C}}$; the arrows and 2arrows in $\mathfrak{C o r r}^{\mathcal{C}}$ are transformations between functors and modifications between 
transformations (see [11]). Any $\mathrm{C}^{*}$-algebra $D$ gives rise to a constant diagram of shape $\mathcal{C}$. The colimit of a diagram $F: \mathcal{C} \rightarrow \mathfrak{C o r r}$ is a $\mathrm{C}^{*}$-algebra colim $F$ with the following universal property:

$$
\mathfrak{C o r r}(\operatorname{colim} F, D) \cong \operatorname{Corr}^{\mathcal{C}}\left(F, \operatorname{const}_{D}\right),
$$

where $\cong$ now means an equivalence of categories.

Let $P$ be a monoid, then a functor $P \rightarrow \mathfrak{C o r r}$ is the same as an essential product system over the monoid $P^{\text {op }}$ (see [17]). We prove in [2] that every diagram in the subcategory of proper correspondences has a colimit; the Cuntz-Pimsner algebra of a proper product system is the colimit of the associated diagram in $\mathfrak{C o r r}$.

Next, let $\mathcal{H}$ and $\mathcal{G}$ be étale groupoids. A correspondence $\mathcal{X}: \mathcal{H} \rightarrow \mathcal{G}$ is a space $\mathcal{X}$ with commuting left and right $\mathcal{G}$-actions on $\mathcal{X}$ such that the right action of $\mathcal{G}$ on $\mathcal{X}$ is free and proper and the source anchor map $s_{\mathcal{X}}: \mathcal{X} \rightarrow \mathcal{G}^{0}$ is a local homeomorphism. We say that $\mathcal{X}$ is proper if the range anchor map $r_{\mathcal{X}}: \mathcal{X} \rightarrow \mathcal{H}^{0}$ induces a proper map $r_{\mathcal{X}_{*}}: \mathcal{X} / \mathcal{G} \rightarrow \mathcal{H}^{0}$. Let $\mathcal{X}: \mathcal{H} \rightarrow \mathcal{G}$ and $\mathcal{Y}: \mathcal{G} \rightarrow \mathcal{D}$ be correspondences. Let $\mathcal{X} \circ_{\mathcal{G}} \mathcal{Y}$ be the quotient of the fibre product $\mathcal{X} \times_{s_{\mathcal{X}}, \mathcal{G}^{0}, r_{\mathcal{Y}}} \mathcal{Y}$ with respect to the diagonal $\mathcal{G}$-action. The space $\mathcal{X} \circ_{\mathcal{G}} \mathcal{Y}$ carries a free and proper right $\mathcal{D}$-action and a left $\mathcal{H}$-action, and the two actions commute. In addition, the anchor map $s: \mathcal{X} \circ_{\mathcal{G}} \mathcal{Y} \rightarrow \mathcal{D}^{0}$ is a local homeomorphism. Thus $\mathcal{X} \circ_{\mathcal{G}} \mathcal{Y}$ is a correspondence from $\mathcal{H}$ to $\mathcal{D}$. We call this correspondence the composite correspondence of $\mathcal{X}$ and $\mathcal{Y}$. We denote by $\mathfrak{G r}$ the bicategory consisting of étale groupoids as objects, groupoid correspondences as arrows and equivariant homeomorphisms of correspondences as 2-arrows. There is a covariant functor $\mathfrak{G r} \rightarrow \mathfrak{C o r r}$ sending a correspondence $\mathcal{X}: \mathcal{H} \rightarrow \mathcal{G}$ to a correspondence $\mathcal{E}: \mathrm{C}^{*}(\mathcal{H}) \rightarrow \mathrm{C}^{*}(\mathcal{G})$.

Colimits in $\mathfrak{G r}$ are defined by an equivalence of groupoids as in Equation (1.2). We construct such colimits for proper Ore monoid actions. And we prove that

$$
\mathrm{C}^{*}\left(\lim _{\longrightarrow}(F)\right)=\underset{\lim }{\longrightarrow} \mathrm{C}^{*}(F)
$$

for such diagrams. This explains groupoid models for row-finite higher-rank graphs without sinks and the $\mathrm{C}^{*}$-algebras of self-similar groups defined by Nekrashevych.

\subsection{Colimit equivalent dynamical systems}

Ever since the introduction of Cuntz-Krieger algebras in [13], many authors have considered the question of deciding which subshifts of finite type have MoritaRieffel equivalent Cuntz-Krieger algebras (see [4, 15,29]). Another related question in symbolic dynamics comes from the conjugacy problem. This classification problem amounts to deciding when two given subshifts are conjugate. A partial answer to this question treats subshifts of finite type as edge shifts of directed 


\section{Introduction}

graphs, see [26, Proposition 2.3.9]. The question can be simplified in terms of defining certain operations on graphs producing conjugacies of edge shifts. Such graphical constructions can be found in the work of Pask and Bates in [5].

Beyond the singly generated dynamics of shifts of finite type, it is more difficult to determine whether two topological dynamical systems give the same $\mathrm{C}^{*}$-algebra. The language of bicategories offers the right setup to understand these questions. An (elementary) shift equivalence in a bicategory can be obtained by a functor from the category $\mathcal{C}_{s}$ generated freely by

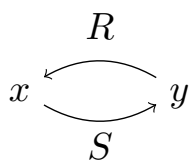

to the bicategory under consideration. In particular, a functor $F: \mathcal{C}_{s} \rightarrow \mathfrak{G r}_{\text {prop }}$ is equivalent to the functor given by two groupoids $\mathcal{G}$ and $\mathcal{H}$ and proper correspondences $\mathcal{X}: \mathcal{G} \rightarrow \mathcal{H}$ and $\mathcal{Y}: \mathcal{H} \rightarrow \mathcal{G}$. The $\mathbb{N}$-actions given by $\mathcal{X} \circ \mathcal{H} \mathcal{Y}: \mathcal{G} \rightarrow \mathcal{G}$ and $\mathcal{Y} \circ \mathcal{G} \mathcal{X}: \mathcal{H} \rightarrow \mathcal{H}$ have the same colimit groupoid in $\mathfrak{G r}$. For the special case where the groupoids $\mathcal{G}$ and $\mathcal{H}$ are just spaces, we obtain the usual notion of elementary strong shift equivalence of topological graphs (compare [44, Definition $2.2])$.

The same happens in the bicategory of $\mathrm{C}^{*}$-correspondences. An elementary strong shift equivalence of $\mathrm{C}^{*}$-correspondences, see [44, Definition 3.10], is equivalent to a functor $F: \mathcal{C}_{s} \rightarrow \mathfrak{C o r r}$.

Both results are special cases of a general phenomenon of colimits of diagrams containing a cofinal subdiagram. This perspective allows to simultaneously treat dynamical systems with the same colimit in $\mathfrak{G r}$ and $\mathfrak{C o r r}$. We call $\left(\mathrm{C}^{*}\right.$-algebraic or topological) dynamical systems colimit equivalent if they have the same colimit.

\subsection{Overview of the thesis}

We describe now the contents of individual chapters of this thesis.

We start in Chapter 2 by recalling basic definitions regarding bicategories. In Section 2.2, we introduce groupoid correspondences and in Section 2.4 we show that groupoid correspondences form a bicategory $\mathfrak{G r}$. In Section 2.5, we construct a functor from $\mathfrak{G r}$ to the bicategory of $\mathrm{C}^{*}$-correspondences $\mathfrak{C o r r}$, and we characterise when the $\mathrm{C}^{*}$-correspondence associated with a groupoid correspondence is proper. In Chapter 2, we also describe functors to $\mathfrak{G r}$ and to $\mathfrak{C o r r}$, transformations between such functors, and modifications between such transformations, and we define colimits of diagrams in $\mathfrak{G r}$. 
In Chapter 3, we introduce and study Ore monoids and Ore monoid actions by proper correspondences and by tight correspondences. Here we say that a correspondence $\mathcal{X}: \mathcal{H} \rightarrow \mathcal{G}$ is tight if the map induced by the range map $r_{\mathcal{X}_{*}}: \mathcal{X} / \mathcal{G} \rightarrow \mathcal{G}^{0}$ is a homeomorphism.

In the first section of Chapter 3, we associate an étale groupoid to a given action of an Ore monoid by tight correspondences. We then show in Theorem 3.15 that this groupoid is the colimit of the action in $\mathfrak{G r}$. We show in Theorem 3.21 that the colimit groupoid of an action in $\mathfrak{G} \mathfrak{r}$ is a groupoid model of the Cuntz-Pimsner algebra for the product system associated with the action.

In Section 3.3 we show how to transform an Ore monoid action by proper correspondences to an action by tight correspondences. This construction preserves the colimits by Theorem 3.30.

Finally, Chapter 4 is devoted to studying diagrams with the same colimit in bicategories. If $\mathcal{C}$ is a diagram with a cofinal object $x$, then the colimit of the diagram $\mathcal{C}$ is isomorphic to the colimit of the diagram $\mathcal{C}(x, x)$. This theorem allows to recover notions like shift equivalence of $\mathrm{C}^{*}$-correspondences, as introduced by [32], as a special case. The same applies to diagrams in the groupoid bicategory. We also generalise the notion of shift equivalence of graphs (see [44]). 
1 Introduction 


\section{Groupoid bicategories and the bicategory of $\mathrm{C}^{*}$-correspondences}

This chapter introduces the bicategory of groupoid correspondences $\mathfrak{G r}$. The definition of a groupoid correspondence is a common generalisation of topological graphs by Katsura [20] and permutational group bimodules [36], which are related to (possibly non-faithful) self-similar group actions. It is based on the notion of groupoid actors by Meyer and Zhu which goes back to Buneci [8]. Our category is a subcategory of the category of topological correspondences introduced in [18]. We construct a morphism from $\mathfrak{G} \mathfrak{r}$ to the bicategory of $\mathrm{C}^{*}$-correspondence $\mathfrak{C o r r}$ as we will see in Section 2.5.

In Section 2.6 we discuss colimits of diagrams in the bicategories $\mathfrak{G r}$ and $\mathfrak{C o r r}$. We finish the chapter by some examples of colimits in $\mathfrak{G r}$ and $\mathfrak{C o r r}$ including colimits of group actions.

\subsection{Bicategories}

We recall in this section some definitions regarding bicategories. Our main reference here is the small preprint [25]. Readers already familiar with bicategories can skip this section.

When taking the leap from category theory to higher category theory, we weaken equalities from one level to natural transformations at the following level. The new structure is then required to satisfy certain coherence conditions. Our interests lie in the realm of bicategories. A bicategory has three levels: objects, arrows and 2-arrows. The composition in the second level is weakly associative and weakly unital. More concretely,

Definition 2.1. A bicategory $\mathcal{C}$ consists of the following data:

(1) A set of objects $\mathcal{C}^{0}$;

(2) categories $\mathcal{C}(A, B)$ for all objects $A$ and $B \in \mathcal{C}^{0}$;

(3) for all objects $A, B$ and $C \in \mathcal{C}^{0}$, a composition bifunctor

$$
*: \mathcal{C}(B, C) \times \mathcal{C}(A, B) \rightarrow \mathcal{C}(A, C), \quad\left(u_{1}, u_{2}\right) \mapsto u_{1} * u_{2},
$$


2 Groupoid bicategories and the bicategory of $\mathrm{C}^{*}$-correspondences

called the horizontal composition, which commutes with the composition in the categories $\mathcal{C}(A, B)$, that is, it commutes with the vertical composition;

(4) for all objects $A, B \in \mathcal{C}^{0}$ and arrows $f \in \mathcal{C}(A, B)^{0}$, natural isomorphisms called the unitor

$$
\begin{aligned}
& l_{f}: 1_{B} * f \Rightarrow f, \\
& r_{f}: f * 1_{A} \Rightarrow f ;
\end{aligned}
$$

(5) for all objects $A, B, C, D \in \mathcal{C}^{0}$, natural isomorphisms called the associator

$$
\Phi: f_{1} *\left(f_{2} * f_{3}\right) \Rightarrow\left(f_{1} * f_{2}\right) * f_{3}
$$

for all composable arrows $f_{1} \in \mathcal{C}(C, D), f_{2} \in \mathcal{C}(B, C)$ and $f_{3} \in \mathcal{C}(A, B)$.

And we require the following axioms:

(6) for all $A, B, C \in \mathcal{C}^{0}$ and all $f_{2} \in \mathcal{C}(A, B)$ and $f_{1} \in \mathcal{C}(B, C)$ the following diagram commutes:

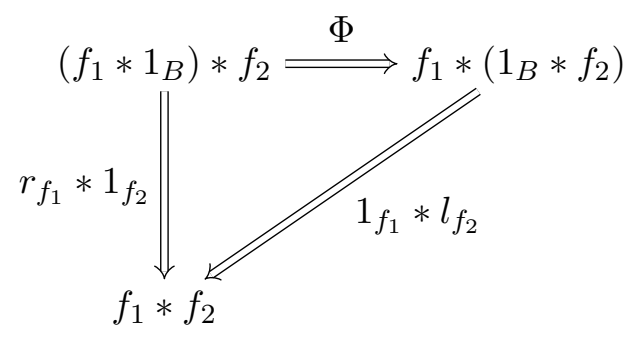

(7) for all composable arrows $f_{1}, f_{2}, f_{3}, f_{4}$ in $\mathcal{C}$ the following diagram commutes:

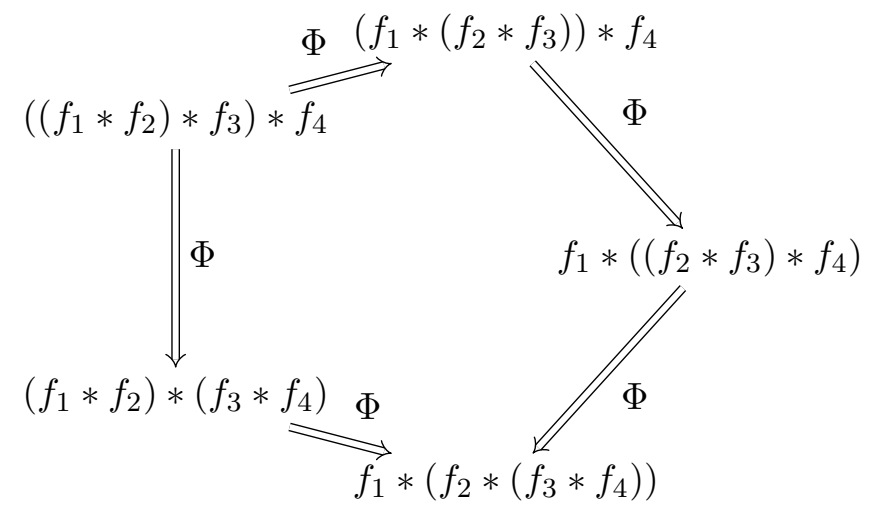


A functor or a morphism of bicategories is a weakening of that of categories. For instance, a functor of bicategories is only unital up to natural transformations.

Definition 2.2. A functor $(\mathcal{F}, \mu): \mathcal{C} \rightarrow \mathcal{D}$ between bicategories consists of the following data

(1) a function $\mathcal{F}_{0}: \mathcal{C}^{0} \rightarrow \mathcal{D}^{0}$,

(2) functors $\mathcal{F}_{1}: \mathcal{C}(A, B) \rightarrow \mathcal{D}\left(\mathcal{F}_{0}(A), \mathcal{F}_{0}(B)\right)$ for all $A$ and $B \in \mathcal{C}^{0}$,

(3) 2-arrows $\mu_{f, g}: F(f) \circ F(g) \Rightarrow F(f \circ g)$ for all composable arrows $f, g \in \mathcal{C}^{1}$,

(4) 2-arrows $\mu_{A}: 1_{F(A)} \Rightarrow F\left(1_{A}\right)$ for all $A \in \mathcal{C}^{0}$,

such that the following diagrams commute:

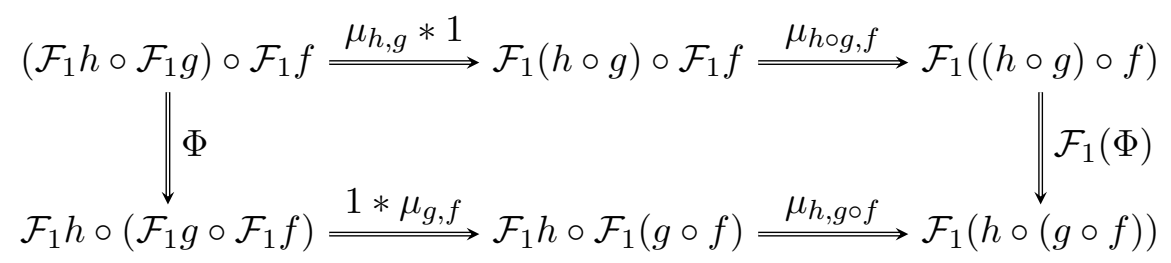

for composable arrows $h, g$ and $f$, and

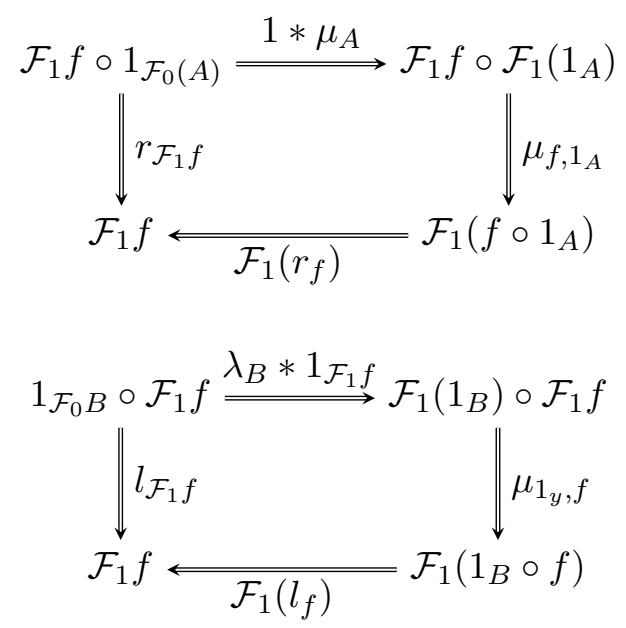

for an arrow $f: A \rightarrow B$ in $\mathcal{C}$.

Definition 2.3. Let $\mathcal{C}$ and $\mathcal{I}$ be bicategories and let $A$ be an object in $\mathcal{C}$. The constant functor const $_{A}: \mathcal{I} \rightarrow \mathcal{C}$ maps all objects $x$ of $\mathcal{I}$ to $A$, all arrows $f$ in $\mathcal{I}$ to the identity arrow on $A$, and the 2 -arrows $\mu$ in Definition 2.1 are identities. 
2 Groupoid bicategories and the bicategory of $\mathrm{C}^{*}$-correspondences

Definition 2.4. Let $\mathcal{C}$ and $\mathcal{D}$ be bicategories and let $(\mathcal{F}, \mu),(\mathcal{J}, \omega): \mathcal{C} \rightrightarrows \mathcal{D}$ be functors. A transformation $\mathcal{F} \Rightarrow \mathcal{J}$ consists of

- arrows $\sigma_{A}: \mathcal{F}_{0}(A) \rightarrow \mathcal{J}_{0}(A)$ for all $A \in \mathcal{C}^{0}$,

- 2-arrows $\sigma_{f}: \mathcal{J}_{1}(f) \circ \sigma_{A} \Rightarrow \sigma_{B} \circ \mathcal{F}_{1}(f)$ for all arrows $f: A \rightarrow B$ in $\mathcal{C}$;

such that the diagram

$$
\begin{gathered}
\left(\mathcal{F}_{1} g \circ \mathcal{F}_{1} f\right) \circ \sigma_{A} \stackrel{\Phi}{\Rightarrow} \mathcal{F}_{1} g \circ\left(\mathcal{F}_{1} f \circ \sigma_{A}\right) \stackrel{1 * \sigma_{f}}{\Rightarrow} \mathcal{F}_{1} g \circ\left(\sigma_{B} \circ \mathcal{J}_{1} f\right) \stackrel{\Phi^{-1}}{\Rightarrow}\left(\mathcal{F}_{1} g \circ \sigma_{B}\right) \circ \mathcal{J}_{1} f \\
\Downarrow \sigma_{g} * 1 \\
\Downarrow \mu_{g, f} * 1 \\
\mathcal{F}_{1}(g \circ f) \circ \sigma_{A} \stackrel{\sigma_{g \circ f}}{\longrightarrow} \sigma_{E} \circ \mathcal{J}_{1}(g \circ f) \underset{1 * \omega_{g, f}}{\longleftarrow} \sigma_{E} \circ\left(\mathcal{J}_{1} g \circ \mathcal{J}_{1} f\right) \underset{\Phi}{\overleftarrow{\Phi}}\left(\sigma_{E} \circ \mathcal{J}_{1} g\right) \circ \mathcal{J}_{1} f
\end{gathered}
$$

commutes for composable arrows $f: A \rightarrow B, g: B \rightarrow E$ in $\mathcal{C}$, and the diagram

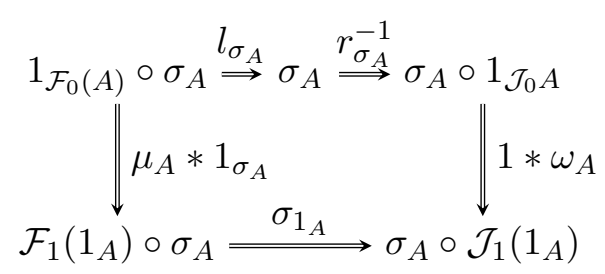

commutes for all objects $A \in \mathcal{C}^{0}$.

We often need to work with transformations to the constant functor over some object in the target bicategory. We rewrite the previous definition in this case.

Definition 2.5. Let $\mathcal{C}$ and $\mathcal{D}$ be bicategories and let $(\mathcal{F}, \mu): \mathcal{C} \rightrightarrows \mathcal{D}$ be a functor and $A \in \mathcal{D}^{0}$. A transformation $\mathcal{F} \Rightarrow$ const $_{A}$ consists of

- arrows $\sigma_{x}: \mathcal{F}_{0}(x) \rightarrow A$ for all $x \in \mathcal{C}^{0}$,

- 2-arrows $\sigma_{f}: \sigma_{x} \Rightarrow \sigma_{y} \circ \mathcal{F}_{1}(f)$ for all arrows $f: x \rightarrow y$ in $\mathcal{C}$;

such that the following diagram commutes for composable arrows $g: y \rightarrow z$, $h: x \rightarrow y:$

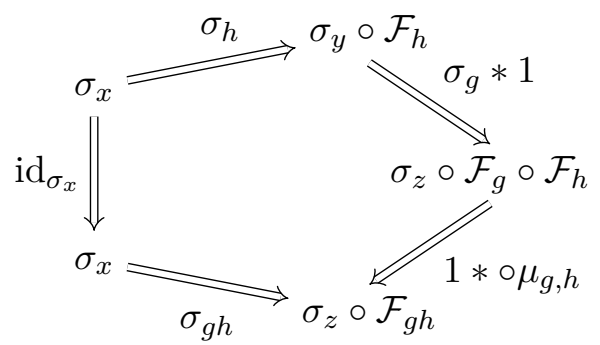


Definition 2.6. Let $\mathcal{C}$ and $\mathcal{D}$ be bicategories and let $(\mathcal{F}, \mu),(\mathcal{J}, \omega): \mathcal{C} \rightrightarrows \mathcal{D}$ be functors. A modification between two transformations $\alpha, \beta: \mathcal{F} \Rightarrow \mathcal{J}$ consists of 2-arrows $\Gamma_{A}: \alpha_{A} \Rightarrow \beta_{A}$ for all objects $A \in \mathcal{C}^{0}$, such that the following diagram commutes for all arrows $f: x \rightarrow y$ in $\mathcal{C}$ :

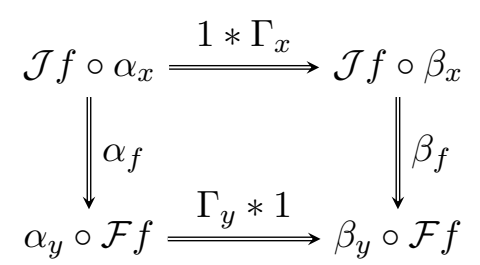

Finally, if $\mathcal{J}$ in Definition 2.6 is the constant diagram over an object $A \in \mathcal{D}^{0}$, then the coherence condition (2.9) simplifies to

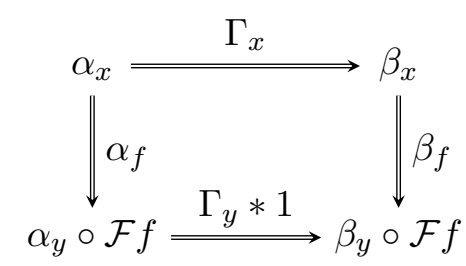

for all arrows $f: x \rightarrow y$ in $\mathcal{C}$.

\subsection{Groupoid correspondences}

A groupoid is a common generalisation of groups and spaces where the unique unit of a group is replaced by a space of units and the multiplication is only partially defined. We start this section with some basic definitions and some results concerning groupoids and their actions. Then we define groupoid correspondences. We finish this section with some examples of our correspondences.

Our main reference for general properties of groupoids, groupoid actions and groupoid principal bundles is the survey article [31]. In the this article, groupoids are dealt with in an abstract setting, allowing us to apply results for non-Hausdorff groupoids as well. Another reference is [45].

Definition 2.7. A (discrete) groupoid is a set $\mathcal{G}$ with partially defined product on a set $\mathcal{G}^{2} \subset \mathcal{G} \times \mathcal{G}$ and an inverse map $g \rightarrow g^{-1}$ such that:

(1) if $(a, b),(b, c) \in \mathcal{G}^{2}$ then $(a b, c),(a, b c) \in \mathcal{G}^{2}$ and

$$
(a b) c=a(b c)
$$


2 Groupoid bicategories and the bicategory of $\mathrm{C}^{*}$-correspondences

(2) $\left(g, g^{-1}\right) \in \mathcal{G}^{2}$ for all $g \in \mathcal{G}$, and if $(a, b) \in \mathcal{G}^{2}$ then

$$
a^{-1}(a b)=b, \quad(a b) b^{-1}=a .
$$

The set of units $\mathcal{G}^{0}$ of $\mathcal{G}$ is the subset of elements $g g^{-1}$ for all $g \in \mathcal{G}$. The source map and the range map are defined by

$$
r(g):=g g^{-1}, \quad s(g):=g^{-1} g
$$

for all $g \in \mathcal{G}$, respectively. For $g, h \in \mathcal{G}$ the pair $(g, h)$ is in $\mathcal{G}^{2}$ if and only if $r(h)=s(g)$. For $x \in \mathcal{G}^{0}$ we denote by $\mathcal{G}_{x}$ and $\mathcal{G}^{x}$ the sets $\mathcal{G}_{x}:=s^{-1}(x)$ and $\mathcal{G}^{x}:=r^{-1}(x)$. We say that $\mathcal{G}$ is a topological groupoid if it has a topology such that the product and inversion are continuous.

The two extreme classes of groupoids are spaces and groups. A space is a groupoid with only units and a group is a groupoid with a single unit. To assign a $\mathrm{C}^{*}$-algebra to a topological group $G$, one needs a left-invariant measure on $G$ in order to define a convolution. Similarly, a family of measures on a groupoid is needed to define its $\mathrm{C}^{*}$-algebra. However, unlike for groups, where a unique left-invariant measure always exists, a family of measures must be specified $a$ priori. We are interested mainly in étale groupoids. These generalise discrete groups, and carry a canonical family of counting measures.

Definition 2.8. An étale locally compact groupoid is a groupoid $\mathcal{G}$ with a topology such that

(1) the space of units $\mathcal{G}^{0}$ is Hausdorff and locally compact;

(2) the range and source maps $r, s: \mathcal{G} \rightrightarrows \mathcal{G}^{0}$ are local homeomorphisms;

(3) the multiplication and inverse maps are continuous.

We allow $\mathcal{G}$ to be non-Hausdorff. Any point in $\mathcal{G}$ has a Hausdorff, compact neighbourhood because $r$ is a local homeomorphism and $\mathcal{G}^{0}$ is Hausdorff and locally compact.

Standing assumption 2.9. We shall assume for the rest of this thesis that all groupoids are étale and locally compact.

Let $\mathcal{G}$ be a groupoid. A right $\mathcal{G}$-space is a topological space $\mathcal{X}$, possibly nonHausdorff, with a continuous map $s: \mathcal{X} \rightarrow \mathcal{G}^{0}$, the anchor map, and a continuous map

mult: $\mathcal{X} \times_{s, \mathcal{G}^{0}, r} \mathcal{G} \rightarrow \mathcal{X}, \quad \mathcal{X} \times{ }_{s, \mathcal{G}^{0}, r} \mathcal{G}:=\{(x, g) \in \mathcal{X} \times \mathcal{G} \mid s(x)=r(g)\}$,

which we denote multiplicatively as $\cdot$, such that 
(1) $s(x \cdot g)=s(g)$ for all $x \in \mathcal{X}, g \in \mathcal{G}$ with $s(x)=r(g)$;

(2) $\left(x \cdot g_{1}\right) \cdot g_{2}=x \cdot\left(g_{1} g_{2}\right)$ for all $x \in \mathcal{X}, g_{1}, g_{2} \in \mathcal{G}$ with $s(x)=r\left(g_{1}\right)$ and $s\left(g_{1}\right)=$ $r\left(g_{2}\right)$

(3) $x \cdot s(x)=x$ for all $x \in \mathcal{X}$.

The orbit space $\mathcal{X} / \mathcal{G}$ is the quotient $\mathcal{X} / \sim_{\mathcal{G}}$ with the quotient topology, where $x \sim_{\mathcal{G}} y$ if there is an element $g \in \mathcal{G}$ with $s(x)=r(g)$ and $x \cdot g=y$.

Proposition 2.10 ([31, Proposition 9.34]). The orbit space projection $\mathrm{p}: \mathcal{X} \rightarrow$ $\mathcal{X} / \mathcal{G}$ is open.

Left $\mathcal{G}$-spaces are defined similarly. We always write $s: \mathcal{X} \rightarrow \mathcal{G}^{0}$ for the anchor map in a right action and $r: \mathcal{X} \rightarrow \mathcal{G}^{0}$ for the anchor map in a left action.

Definition 2.11. Let $\mathcal{X}$ and $\mathcal{Y}$ be right $\mathcal{G}$-spaces. A continuous map $f: \mathcal{X} \rightarrow \mathcal{Y}$ is $\mathcal{G}$-equivariant if $s(f(x))=s(x)$ for all $x \in \mathcal{X}$ and $f(x \cdot g)=f(x) \cdot g$ for all $x \in \mathcal{X}, g \in \mathcal{G}$ with $s(x)=r(g)$.

Let $\mathcal{X}$ be a right $\mathcal{G}$-space and $\mathcal{Z}$ a space. A continuous map $f: \mathcal{X} \rightarrow \mathcal{Z}$ is $\mathcal{G}$-invariant if $f(x \cdot g)=f(x)$ for all $x \in \mathcal{X}, g \in \mathcal{G}$ with $s(x)=r(g)$.

Definition 2.12 ([7, I.10.1], "application propre"). Let $X$ and $Y$ be topological spaces. A map $f: X \rightarrow Y$ is proper if $f \times \operatorname{id}_{Z}: X \times Z \rightarrow Y \times Z$ is closed for every topological space $Z$.

Definition 2.13 ([45, Definition 2.9]). A groupoid $\mathcal{G}$ is proper if the following map is proper:

$$
(r, s): \mathcal{G} \rightarrow \mathcal{G}^{0} \times \mathcal{G}^{0}, \quad g \mapsto(r(g), s(g)) .
$$

Definition 2.14. A right $\mathcal{G}$-space $\mathcal{X}$ is proper if the transformation groupoid $\mathcal{X} \rtimes \mathcal{G}$ is proper, that is, the following map is proper:

$$
\mathcal{X} \times_{s, \mathcal{G}^{0}, r} \mathcal{G} \rightarrow \mathcal{X} \times \mathcal{X}, \quad(x, g) \mapsto(x \cdot g, x) .
$$

Definition 2.15. A right $\mathcal{G}$-space $\mathcal{X}$ is basic if the map in (2.11) is a homeomorphism onto its image.

The image of the map (2.11) is the subspace

$$
\mathcal{X} \times{ }_{\mathcal{X} / \mathcal{G}} \mathcal{X}:=\left\{(x, y) \in \mathcal{X}^{2} \mid x \sim_{\mathcal{G}} y\right\} .
$$

Basic actions are defined in [31] in a more abstract setting, and the definition above is an equivalent characterisation of the basic actions in the setting of topological spaces, possibly non-Hausdorff, with étale surjections as covers, compare [31, Proposition 9.40]. We recall some more details related to this. 
2 Groupoid bicategories and the bicategory of $\mathrm{C}^{*}$-correspondences

Definition 2.16. A right $\mathcal{G}$-space $\mathcal{X}$ is free if $x \cdot g=x$ for $x \in \mathcal{X}$ and $g \in \mathcal{G}$ implies $g=1_{s(x)}$.

An action is free if and only if the map (2.11) is injective. An action is basic if and only if it is free and the map from $\mathcal{X} \times \mathcal{X} / \mathcal{G} X$ to $\mathcal{G}$ that maps $(x, y) \in \mathcal{X}^{2}$ with $x \sim_{\mathcal{G}} y$ to the unique $g \in \mathcal{G}$ with $s(x)=r(g)$ and $x \cdot g=y$ is continuous: this

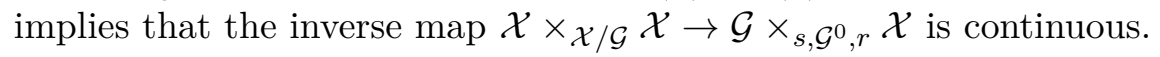

Proposition 2.17 ([31, Corollary 9.35 and Proposition 9.40]). Let $\mathcal{G}$ be a groupoid and $\mathcal{X}$ a right $\mathcal{G}$-space. The following are equivalent:

(1) the action of $\mathcal{G}$ on $\mathcal{X}$ is basic and the orbit space $\mathcal{X} / \mathcal{G}$ is Hausdorff;

(2) the action of $\mathcal{G}$ on $\mathcal{X}$ is free and proper.

In this case, the orbit space projection is a local homeomorphism.

The statement in Proposition 2.17 about the orbit space projection needs our standing assumption that $\mathcal{G}$ is étale.

All topological spaces that we shall need are locally Hausdorff and locally compact.

\subsubsection{Groupoid correspondences}

We introduce the main building blocks of our bicategory: groupoid correspondences.

Definition 2.18. Let $\mathcal{H}$ and $\mathcal{G}$ be groupoids. A groupoid correspondence from $\mathcal{H}$ to $\mathcal{G}$ is a (topological) space $\mathcal{X}$ with commuting actions of $\mathcal{H}$ on the left and $\mathcal{G}$ on the right, such that the right anchor map $s: \mathcal{X} \rightarrow \mathcal{G}^{0}$ is a local homeomorphism and the right $\mathcal{G}$-action is free and proper.

Having commuting actions of $\mathcal{G}$ and $\mathcal{H}$ means that $s(h \cdot x)=s(x), r(x \cdot g)=r(x)$, and $(h \cdot x) \cdot g=h \cdot(x \cdot g)$ for all $g \in \mathcal{G}, x \in \mathcal{X}, h \in \mathcal{H}$ with $s(h)=r(x)$ and $s(x)=r(g)$, where $s: \mathcal{X} \rightarrow \mathcal{G}^{0}$ and $r: \mathcal{X} \rightarrow \mathcal{H}^{0}$ are the anchor maps. If $s: \mathcal{X} \rightarrow \mathcal{G}^{0}$ is a local homeomorphism and $\mathcal{G}^{0}$ is Hausdorff, locally compact, then $\mathcal{X}$ must be locally Hausdorff and locally compact; but it need not be Hausdorff. The orbit space $\mathcal{X} / \mathcal{G}$ is Hausdorff and the projection map $\mathcal{X} \rightarrow \mathcal{X} / \mathcal{G}$ is a local homeomorphism by Proposition 2.17. Thus $\mathcal{X} / \mathcal{G}$ is locally compact as well.

Definition 2.19. A correspondence $\mathcal{X}: \mathcal{H} \rightarrow \mathcal{G}$ is proper if its left anchor map induces a proper map $r_{*}: \mathcal{X} / \mathcal{G} \rightarrow \mathcal{H}^{0}$. It is tight if $r_{*}$ is a homeomorphism.

Lemma 2.20. For a tight correspondence $\mathcal{X}: \mathcal{H} \rightarrow \mathcal{G}$ the map $r: \mathcal{X} \rightarrow \mathcal{H}^{0}$ is a surjective local homeomorphism. 
Proof. The quotient map $\mathcal{X} \rightarrow \mathcal{X} / \mathcal{G}$ is a surjective local homeomorphism by Proposition 2.17, and $r_{*}: \mathcal{X} / \mathcal{G} \rightarrow \mathcal{H}^{0}$ is a homeomorphism. The map $r$ is the product of these two maps and hence also a surjective local homeomorphism.

In [31], a "bibundle actor" between two groupoids in a category with a pretopology is defined as an object in the underlying category with two commuting actions such that the right action is basic and the right anchor map is a cover in the pretopology. In particular, a bibundle actor in the category of locally Hausdorff, locally compact spaces with surjective local homeomorphisms as covers is a space $\mathcal{X}$ with two commuting actions of $\mathcal{H}$ and $\mathcal{G}$ such that the right anchor map $s$ is a surjective local homeomorphism and the right action is basic. This is very close to a groupoid correspondence. There are only two differences. First, we do not require the source map on $\mathcal{X}$ to be surjective because we never need this and there are relevant examples where this does not happen. Secondly, we ask the orbit space $\mathcal{X} / \mathcal{G}$ to be Hausdorff. (By Proposition 2.17, an action is free and proper if and only if it is basic and the orbit space is Hausdorff.) Furthermore, for a bibundle actor as above, the groupoids involved may have a locally Hausdorff, locally compact object space. We need $\mathcal{X} / \mathcal{G}$ to be Hausdorff to ensure that certain groupoids we are going to construct from a correspondence have a Hausdorff object space and also to pass to $\mathrm{C}^{*}$-correspondences.

The tight bibundle actors are close to the covering bibundle functors in the notation of [31]. Here the only difference is that we do not require the right anchor map to be surjective. The orbit space $\mathcal{X} / \mathcal{G}$ is required, anyway, to be homeomorphic to $\mathcal{H}^{0}$, so its Hausdorffness is not an assumption on $\mathcal{X}$ but only on $\mathcal{H}$.

Definition 2.21. A correspondence $\mathcal{X}: \mathcal{G} \rightarrow \mathcal{H}$ is called a bicorrespondence if the range map $r: \mathcal{X} \rightarrow \mathcal{G}$ is a local homeomorphism and the action of $\mathcal{G}$ on $\mathcal{X}$ is free and proper. A bicorrespondence $\mathcal{X}: \mathcal{G} \rightarrow \mathcal{H}$ is called a partial equivalence if $r_{*}: \mathcal{X} / \mathcal{H} \rightarrow \mathcal{G}^{0}$ and $s_{*}: \mathcal{G} \backslash \mathcal{X} \rightarrow \mathcal{H}^{0}$ are homeomorphisms onto their images.

\subsubsection{Examples}

Example 2.22. Let $G$ and $H$ be locally compact spaces viewed as groupoids with no arrows and let $X: H \rightarrow G$ be a correspondence. Since the spaces $G$ and $H$ act trivially on $X$, the correspondence $X$ is equivalent to the triple $(X, r, s)$. That is, the correspondence $X$ is given by a locally compact space $X$ with anchor maps $s: X \rightarrow G$ and $r: X \rightarrow H$ such that $s$ is a local homeomorphism. The triple $(X, r, s)$ is called a topological correspondence in [1]. See also [20]. It is called a continuous graph if $G$ and $H$ are the same space and $r: X \rightarrow H$ is also a local homeomorphism (see [14]). Hence a continuous graph is a bicorrespondence over a locally compact space. 
2 Groupoid bicategories and the bicategory of $\mathrm{C}^{*}$-correspondences

Example 2.23. Let $X$ be a finite set and let $G$ be a discrete group acting on $X$. Let $\varphi: G \times X \rightarrow G$ be a (one)-cocycle for this action. That is, for all $g, h \in G$ and all $x \in X$ we have

$$
\varphi(g h, x)=\varphi(g, h(x)) \varphi(h, x) .
$$

Let $X^{*}$ be the set of finite words over $X$. The action of $G$ on $X$ extends to an action on $X^{*}$ using the recursive formula

$$
g(x w)=g(x) \varphi(g, x)(w)
$$

for all $g, h \in G, x \in X$ and all $w \in X^{*}$. We call the triple $(G, X, \varphi)$ a self-similar action of the group $G$.

The pair $(G, X)$ is called a faithful self-similar action if the action of $G$ on $X^{*}$ is faithful (compare [37, Definition 2.1]). In this case, $\varphi(g, x)$ is called the restriction of $g$ on $x$ and denoted $\left.g\right|_{x}$.

Lemma 2.24. Let $G$ be a discrete group. Self-similar $G$-actions are equivalent to proper correspondences over $G$.

Proof. Let $(G, X, \varphi)$ be a self-similar group. The space $\mathcal{X}:=X \times G$ is in a natural way a correspondence over the group $G$. The left and right actions of $G$ on $X \times G$ are

$$
\begin{aligned}
h \cdot(x, g) & :=(h(x), \varphi(h, x) g), \\
(x, g) \cdot h & :=(x, g h)
\end{aligned}
$$

for all $x \in X$ and all $g, h \in G$. Clearly, the anchor map $s: X \times G \rightarrow\left\{G^{0}\right\}=\{1\}$ is a local homeomorphism since $X \times G$ is discrete. Furthermore, the right action is free and proper. In fact, the map $((x, g), h) \mapsto(x, g h)$ is a homeomorphism. The orbit space of the right action $\mathcal{X} / G$ is homeomorphic to $X$ via the map $[x, g] \mapsto x$. The map induced by the range map $r_{*}: \mathcal{X} / G \rightarrow\{1\}$ is proper by our assumption that $X$ is finite.

Conversely, let $\mathcal{X}: G \rightarrow G$ be a proper correspondence. The source anchor map $s: \mathcal{X} \rightarrow\{1\}$ is a local homeomorphism. Thus $\mathcal{X}$ is discrete. The map $r_{*}: \mathcal{X} / G \rightarrow\{1\}$ is proper. Hence $\mathcal{X} / G$ is compact and so it is finite. Therefore, we may choose a finite fundamental domain (a basis) $X \subset \mathcal{X}$ for the right action. That is, $X$ intersects every orbit of the right action once. By assumption, the right action is free. Hence the restriction of the multiplication map $m: \mathcal{X} \times G \rightarrow \mathcal{X}$ to $X \times G$ is an invertible map. Let $\theta: \mathcal{X} \rightarrow X \times G$ be its inverse. That is, if $\theta(y)=(x, g)$ for an element $y \in \mathcal{X}$, then the elements $x \in X$ and $g \in G$ are the unique elements with $y=x \cdot g$. The space $X \times G$ is a right $G$-space. Furthermore, 
the map $\theta$ is $G$-equivariant. That is, $\theta(y g)=\theta(y) g$ for all $y \in \mathcal{X}$ and all $g \in G$. We define a left action of $G$ on the space $X \times G$ by the rule

$$
g \theta(y)=\theta(g y)
$$

for all $y \in \mathcal{X}$. With this action, $X \times G$ becomes a correspondence over the group $G$ such that $\theta: \mathcal{X} \rightarrow X \times G$ is an isomorphism. The map

$$
X \rightarrow X, \quad x \mapsto \operatorname{pr}_{1}(\theta(g \cdot x)),
$$

for all $g \in G$, defines a permutation action of the group $G$ on the finite set $X$. We define

$$
\varphi(g, x):=\operatorname{pr}_{2}(\theta(g \cdot x))
$$

for all $g \in G$ and $x \in X$. Then

$$
\varphi(g h, x)=\varphi(g, h(x)) \varphi(h, x)
$$

for all $g, h \in G$ and $x \in X$. That is, the map

$$
G \times X \rightarrow G, \quad(x, g) \mapsto \varphi(g, x)
$$

is a cocycle. The triple $(X, G, \varphi)$ is a self-similar group. We have shown that the map $\theta: \mathcal{X} \rightarrow X \times G$ is an isomorphism of correspondences. So the two constructions are inverse to each other up to isomorphism.

A proper correspondence over a discrete group is the same as a $d$-covering permutational bimodule over the group as in [36, §2.1].

Lemma 2.24 gives an equivalence between self-similar group actions and proper group correspondences. A closer investigation shows that a tight group correspondence is equivalent to a self-similar action on an alphabet consisting of one letter. That is, a tight correspondence over a group $G$ is equivalent to a group endomorphism over $G$.

Example 2.25. Let $G$ and $H$ be discrete groups. Let $\varphi: H \rightarrow G$ be a group homomorphism. Let $G_{\varphi}:=G$ as a set. Then $G_{\varphi}$ is a free and proper right $G$-space with the group multiplication and the trivial anchor map. The group $H$ acts on $G_{\varphi}$ using the maps $\varphi$. That is, for all $h \in H$ and $g \in G_{\varphi}$ we define

$$
h \cdot g:=\varphi(h) g .
$$

This action commutes with the action of $G$ on itself by right multiplication and hence $G_{\varphi}$ is a correspondence. Moreover, $G_{\varphi} / G=\left[1_{G}\right]$, which implies that $G_{\varphi}: H \rightarrow G$ is tight. Conversely, any tight correspondence $\mathcal{X}: H \rightarrow G$ is isomorphic to a correspondence of the form $G_{\psi}$ for some homomorphism $\psi: H \rightarrow G$. 
2 Groupoid bicategories and the bicategory of $\mathrm{C}^{*}$-correspondences

To see this, first observe that $|\mathcal{X} / G|=1$. We fix an element $x \in \mathcal{X}$. Then for all $y \in \mathcal{X}$ there is a unique element $g=\langle x, y\rangle$ such that $y=x g$. This implies that

$$
L_{x}: G \rightarrow \mathcal{X}, \quad g \mapsto x g,
$$

is a bijection. Now let $h \in H$. There is a unique element $\varphi(h, x) \in G$ such that $h(x)=x \varphi(h, x)$. We define

$$
\psi(h):=\varphi(h, x)
$$

for all $h \in H$. Then $\psi$ is a group homomorphism.

\subsection{The composition of groupoid correspondences}

Our next step toward a bicategory of groupoid correspondences is the composition bifunctor. We will compose two correspondences $\mathcal{X}: \mathcal{G} \rightarrow \mathcal{H}$ and $\mathcal{Y}: \mathcal{H} \rightarrow \mathcal{K}$ to obtain a correspondence $\mathcal{G} \rightarrow \mathcal{K}$. The classes of tight and proper correspondences are closed under this composition.

Let $\mathcal{X}: \mathcal{H} \rightarrow \mathcal{G}$ be a groupoid correspondence. The image of the map (2.11) consists of those $\left(\gamma_{1}, \gamma_{2}\right) \in \mathcal{X} \times \mathcal{X}$ with $\left[\gamma_{1}\right]=\left[\gamma_{2}\right]$ in $\mathcal{X} / \mathcal{G}$. Since $(2.11)$ is a homeomorphism onto its image for any groupoid correspondence, we may define a continuous map

$$
\mathcal{X} \times \mathcal{X} / \mathcal{G} \mathcal{X} \stackrel{\sim}{\rightarrow} \mathcal{X} \times_{s, \mathcal{G}^{0}, r} \mathcal{G} \stackrel{\mathrm{pr}_{2}}{\longrightarrow} \mathcal{G}, \quad\left(\gamma_{1}, \gamma_{2}\right) \mapsto\left\langle\gamma_{1}, \gamma_{2}\right\rangle .
$$

That is, $\left\langle\gamma_{1}, \gamma_{2}\right\rangle$ for $\gamma_{1}, \gamma_{2} \in \mathcal{X}$ with $\left[\gamma_{1}\right]=\left[\gamma_{2}\right]$ in $\mathcal{X} / \mathcal{G}$ is the unique $\eta \in \mathcal{G}$ with $\gamma_{1} \eta=\gamma_{2}$.

Proposition 2.26. Let $\mathcal{X}: \mathcal{H} \rightarrow \mathcal{G}$ be a groupoid correspondence. The inner product map in (2.14) is a local homeomorphism. It has the following properties:

(1) $s\left(\left\langle\gamma_{1}, \gamma_{2}\right\rangle=s\left(\gamma_{2}\right), r\left(\left\langle\gamma_{1}, \gamma_{2}\right\rangle=s\left(\gamma_{1}\right)\right.\right.$, and $\gamma_{1} \cdot\left\langle\gamma_{1}, \gamma_{2}\right\rangle=\gamma_{2}$ for all $\gamma_{1}, \gamma_{2} \in \mathcal{X}$ with $\left[\gamma_{1}\right]=\left[\gamma_{2}\right]$;

(2) $\langle\gamma, \gamma\rangle=1$ for all $\gamma \in \mathcal{X}$;

(3) $\left\langle\gamma_{2}, \gamma_{1}\right\rangle=\left\langle\gamma_{1}, \gamma_{2}\right\rangle^{-1}$ for all $\gamma_{1}, \gamma_{2} \in \mathcal{X}$ with $\left[\gamma_{1}\right]=\left[\gamma_{2}\right]$;

(4) $\left\langle\zeta \gamma_{1} \eta_{1}, \zeta \gamma_{2} \eta_{2}\right\rangle=\eta_{1}^{-1}\left\langle\gamma_{1}, \gamma_{2}\right\rangle \eta_{2}$ for all $\zeta \in \mathcal{H}, \gamma_{1}, \gamma_{2} \in \mathcal{X}, \eta_{1}, \eta_{2} \in \mathcal{G}$ with $s(\zeta)=r\left(\gamma_{1}\right)=r\left(\gamma_{2}\right), s\left(\gamma_{1}\right)=r\left(\eta_{1}\right), s\left(\gamma_{2}\right)=r\left(\eta_{2}\right),\left[\gamma_{1}\right]=\left[\gamma_{2}\right]$.

Proof. The coordinate projection $\operatorname{pr}_{2}: \mathcal{X} \times_{s, \mathcal{G}^{0}, r} \mathcal{G} \rightarrow \mathcal{G}$ is a local homeomorphism because $s: \mathcal{X} \rightarrow \mathcal{G}^{0}$ is a local homeomorphism. Hence so is the map in (2.14). The algebraic properties of the inner product map are verified by direct computation. 
Let $\mathcal{H}, \mathcal{G}$ and $\mathcal{K}$ be groupoids and let $\mathcal{X}: \mathcal{H} \rightarrow \mathcal{G}$ and $\mathcal{Y}: \mathcal{G} \rightarrow \mathcal{K}$ be groupoid correspondences. We are going to compose them. Let

$$
\mathcal{X} \times \mathcal{G}^{0} \mathcal{Y}:=\{(x, y) \in \mathcal{X} \times \mathcal{Y} \mid s(x)=r(y)\} .
$$

We define an action of $\mathcal{G}$ on the space $\mathcal{X} \times \mathcal{G}^{0} \mathcal{Y}$ by

$$
g \cdot(x, y):=\left(x \cdot g^{-1}, g \cdot y\right)
$$

for $x \in \mathcal{X}, y \in \mathcal{Y}$ and $g \in \mathcal{G}$ with $s(g)=r_{\mathcal{Y}}(y)=s_{\mathcal{X}}(x)$. We call this the diagonal action. Let $\mathcal{X} \circ_{\mathcal{G}} \mathcal{Y}$ be the orbit space of the diagonal action. The maps $r(x, y):=r_{\mathcal{X}}(x)$ and $s(x, y):=s_{\mathcal{Y}}(y)$ on $\mathcal{X} \times_{\mathcal{G}^{0}} \mathcal{Y}$ induce maps $r: \mathcal{X} \circ_{\mathcal{G}} \mathcal{Y} \rightarrow$ $\mathcal{H}^{0}$ and $s: \mathcal{X} \circ_{\mathcal{G}} \mathcal{Y} \rightarrow \mathcal{K}^{0}$. These are the anchor maps for commuting actions of $\mathcal{H}$ on the left and $\mathcal{K}$ on the right:

$$
h \cdot[x, y]:=[h \cdot x, y], \quad[x, y] \cdot k:=[x, y \cdot k],
$$

for all $h \in \mathcal{H}, x \in \mathcal{X}, y \in \mathcal{Y}, k \in \mathcal{K}$ with $s(h)=r(x), s(x)=r(y)$, and $s(y)=r(k)$. Here $[x, y]$ denotes the image of $(x, y) \in \mathcal{X} \times_{\mathcal{G}^{0}} \mathcal{Y}$ in $\mathcal{X} \circ_{\mathcal{G}} \mathcal{Y}$.

Proposition 2.27. The actions of $\mathcal{H}$ and $\mathcal{K}$ on $\mathcal{X} \circ_{\mathcal{G}} \mathcal{Y}$ defined above are well defined and turn this into a groupoid correspondence $\mathcal{H} \rightarrow \mathcal{K}$.

If both correspondences $\mathcal{X}$ and $\mathcal{Y}$ are proper or tight or bicorrespondences or partial equivalences, then so is $\mathcal{X} \circ_{\mathcal{G}} \mathcal{Y}$.

Proof. The action of $\mathcal{G}$ on $\mathcal{X}$ is basic and the coordinate projection $\mathcal{X} \times \mathcal{G}^{0} \mathcal{Y} \rightarrow$ $\mathcal{X}$ is $\mathcal{G}$-equivariant. Therefore, the diagonal action of $\mathcal{G}$ on $\mathcal{X} \times \mathcal{G}^{0} \mathcal{Y}$ is also basic. Arguments as for the composition of bibundle actors in [31] show that the induced actions of $\mathcal{H}$ and $\mathcal{K}$ are well-defined, continuous groupoid actions on the space $\mathcal{X} \circ_{\mathcal{G}} \mathcal{Y}$, that the source map $s: \mathcal{X} \circ_{\mathcal{G}} \mathcal{Y} \rightarrow \mathcal{K}^{0}$ is a local homeomorphism, and that the right $\mathcal{K}$-action is basic. One way to show this is by shrinking $\mathcal{G}$ and $\mathcal{K}$ to the open subgroupoids with object spaces $s(\mathcal{X})$ and $s_{\mathcal{Y}}\left(r_{\mathcal{Y}}^{-1}\left(s_{\mathcal{X}}(\mathcal{X})\right)\right.$, respectively, to make the two right anchor maps surjective. Then our groupoid correspondences become bibundle actors in a suitable category with pretopology, which satisfies the axioms in [31] that show that the composite is again a bibundle actor.

We claim that the orbit space of the $\mathcal{K}$-action on $\mathcal{X} \circ_{\mathcal{G}} \mathcal{Y}$ is Hausdorff. The orbit space $\mathcal{Y} / \mathcal{K}$ is Hausdorff by Proposition 2.17, and the right $\mathcal{G}$-action on $\mathcal{X}$ is proper. Hence the diagonal action of $\mathcal{G}$ on $\mathcal{X} \times \mathcal{G}^{0}(\mathcal{Y} / \mathcal{K})$ is proper by [45, Proposition 2.20]. Thus the orbit space of this proper action is Hausdorff by Proposition 2.17. This orbit space is $\mathcal{X} \circ_{\mathcal{G}}(\mathcal{Y} / \mathcal{K}) \cong\left(\mathcal{X} \circ_{\mathcal{G}} \mathcal{Y}\right) / \mathcal{K}$ by [31, Remark 7.9]. Since the right $\mathcal{K}$-action on $\mathcal{X} \circ_{\mathcal{G}} \mathcal{Y}$ is basic and its orbit space is Hausdorff, this action is free and proper by Proposition 2.17. Thus $\mathcal{X} \circ_{\mathcal{G}} \mathcal{Y}$ is a groupoid correspondence. 
2 Groupoid bicategories and the bicategory of $\mathrm{C}^{*}$-correspondences

Now assume that the groupoid correspondences $\mathcal{X}$ and $\mathcal{Y}$ are proper. We want to prove that their product remains proper. We use the following pull-back diagram:

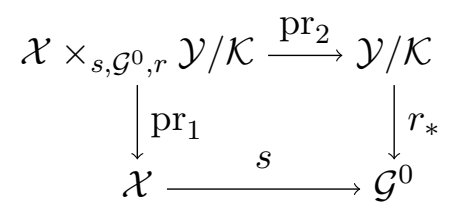

The map $r_{*}: \mathcal{Y} / \mathcal{K} \rightarrow \mathcal{G}^{0}$ is proper. Hence the parallel map $\mathrm{pr}_{1}$ in the pull-back diagram is also proper. Then the induced map $\left(\mathrm{pr}_{1}\right)_{*}: \mathcal{X} \circ \mathcal{G} \mathcal{Y} / \mathcal{K} \rightarrow \mathcal{X} / \mathcal{G}$ on the orbit spaces is proper by [45, Lemma 2.32]. The map $r_{*}: \mathcal{X} \circ_{\mathcal{G}} \mathcal{Y} / \mathcal{K} \rightarrow \mathcal{H}^{0}$ is proper because it is the product of the proper map $r_{*}: \mathcal{X} / \mathcal{G} \rightarrow \mathcal{H}^{0}$ with $\left(\operatorname{pr}_{1}\right)_{*}$.

Assume that the groupoid correspondences $\mathcal{X}$ and $\mathcal{Y}$ are tight. That is, the maps $r_{*}: \mathcal{X} / \mathcal{G} \rightarrow \mathcal{H}^{0}$ and $r_{*}: \mathcal{Y} / \mathcal{K} \rightarrow \mathcal{G}^{0}$ are homeomorphisms. The pullback diagram (2.15) shows that $\left(\mathrm{pr}_{1}\right)_{*}: \mathcal{X} \times_{\mathcal{G}^{0}} \mathcal{Y} / \mathcal{K} \rightarrow \mathcal{X} / \mathcal{G}$ is a homeomorphism. Then the induced map $\left(\mathrm{pr}_{1}\right)_{*}: \mathcal{X} \circ_{\mathcal{G}} \mathcal{Y} / \mathcal{K} \rightarrow \mathcal{X} / \mathcal{G}$ is a homeomorphism by $[31$, Proposition 5.9]. The map $r_{*}: \mathcal{X} \circ_{\mathcal{G}} \mathcal{Y} / \mathcal{K} \rightarrow \mathcal{H}^{0}$ is a homeomorphism because it is the product of the homeomorphism $r_{*}: \mathcal{X} / \mathcal{G} \rightarrow \mathcal{H}^{0}$ with $\left(\mathrm{pr}_{1}\right)_{*}$. Finally, using the same arguments as before, $\mathcal{X} \circ \mathcal{G} \mathcal{Y}$ is a bicorrespondences, or a partial equivalence if both $\mathcal{X}$ and $\mathcal{Y}$ are.

The next two lemmas are proved like their analogues for bibundle actors in [31].

Lemma 2.28. Let $\mathcal{X}: \mathcal{H} \rightarrow \mathcal{G}$ be a groupoid correspondence. The maps

$$
\begin{aligned}
\mathcal{H} \circ_{\mathcal{H}} \mathcal{X} & \rightarrow \mathcal{X}, & {[h, x] } & \mapsto h \cdot x, \\
\mathcal{X} \circ_{\mathcal{G}} \mathcal{G} & \rightarrow \mathcal{X}, & {[x, g] } & \mapsto x \cdot g,
\end{aligned}
$$

are $\mathcal{G}, \mathcal{H}$-equivariant homeomorphisms.

Lemma 2.29. Let $\mathcal{G}_{i}$ for $1 \leq i \leq 4$ be groupoids. Let $\mathcal{X}_{i}: \mathcal{G}_{i} \rightarrow \mathcal{G}_{i+1}$ for $1 \leq i \leq 3$ be correspondences. The map

$$
\mathcal{X}_{1} \circ_{\mathcal{G}_{1}}\left(\mathcal{X}_{2} \circ_{\mathcal{G}_{2}} \mathcal{X}_{3}\right) \rightarrow\left(\mathcal{X}_{1} \circ_{\mathcal{G}_{1}} \mathcal{X}_{2}\right) \circ_{\mathcal{G}_{2}} \mathcal{X}_{3}, \quad\left[x_{1},\left[x_{2}, x_{3}\right]\right] \mapsto\left[\left[x_{1}, x_{2}\right], x_{3}\right],
$$

is a $\mathcal{G}_{1}, \mathcal{G}_{4}$-equivariant homeomorphism.

\subsection{Bicategories of groupoid correspondences}

In this section, we show that correspondences form a bicategory $\mathfrak{G r}$; the subclasses of proper or tight correspondences and the subclasses of bicorrespondences and partial equivalences are all subbicategories in $\mathfrak{G} \mathfrak{r}$. 
We define the bicategory of groupoid correspondences $\mathfrak{G r}$. Its objects are (étale, locally compact, possibly non-Hausdorff) groupoids. Its arrows $\mathcal{H} \rightarrow \mathcal{G}$ are groupoid correspondences $\mathcal{H} \rightarrow \mathcal{G}$. The 2 -arrows $\mathcal{X} \Rightarrow \mathcal{Y}$ for arrows $\mathcal{X}, \mathcal{Y}: \mathcal{G} \rightrightarrows$ $\mathcal{H}$ are $\mathcal{G}, \mathcal{H}$-equivariant homeomorphisms $\mathcal{X} \cong \mathcal{Y}$. The composition of arrows $\mathcal{H} \rightarrow \mathcal{G} \rightarrow \mathcal{K}$ is the construction $\circ_{\mathcal{G}}$ above, which we often abbreviate from now on as $\circ$. This is a bifunctor with respect to our 2-arrows, that is, equivariant homeomorphisms on both factors. The unit transformations and associator are given by Lemma 2.28 and Lemma 2.29. These are clearly natural for equivariant homeomorphisms of groupoid correspondences, and they also satisfy the coherence conditions needed for a bicategory, see Definition 2.1.

Theorem 2.30. The equivalences in $\mathfrak{G r}$ are exactly the Morita equivalences of groupoids. That is, a groupoid correspondence $\mathcal{X}: \mathcal{H} \rightarrow \mathcal{G}$ is an equivalence if and only if both actions are basic and both anchor maps induce homeomorphisms $\mathcal{X} / \mathcal{G} \cong$ $\mathcal{H}^{0}$ and $\mathcal{H} \backslash \mathcal{X} \cong \mathcal{G}^{0}$.

Proof. First, let $\mathcal{X}$ be a Morita equivalence $\mathcal{H} \rightarrow \mathcal{G}$. Then $\mathcal{X}$ is a groupoid correspondence as well, and so is the inverse Morita equivalence $\mathcal{X}^{*}$, which we get by reversing left and right in $\mathcal{X}$. Being a Morita equivalence implies $\mathcal{X} \circ \mathcal{X}^{*} \cong \mathcal{H}$ and $\mathcal{X}^{*} \circ \mathcal{X} \cong \mathcal{G}$. Thus $\mathcal{X}$ is an equivalence in $\mathfrak{G r}$.

Conversely, let $\mathcal{X}: \mathcal{H} \rightarrow \mathcal{G}$ be an equivalence in $\mathfrak{G r}$ with inverse $\mathcal{Y}$. Since the right anchor map $s: \mathcal{Y} \circ \mathcal{X} \rightarrow \mathcal{G}^{0}$ is surjective, the right anchor map $s: \mathcal{X} \rightarrow \mathcal{G}^{0}$ is surjective. Similarly, the right anchor map $s: \mathcal{Y} \rightarrow \mathcal{H}^{0}$ is surjective. Thus both $\mathcal{X}$ and $\mathcal{Y}$ are bibundle actors in a suitable category with pretopology, and so they are equivalences in the bicategory of bibundle actors. These equivalences are identified with bibundle equivalences in [31, Theorem 7.31]. These are exactly the Morita equivalences.

The classes of proper and tight correspondences and the classes of bicorrespondences and partial equivalences are all closed under composition by Proposition 2.27. In addition, the identity correspondence over a groupoid $\mathcal{G}$ belongs to all three classes. Therefore, these classes are subbicategories in $\mathfrak{G} \mathfrak{r}$. We denote these by $\mathfrak{G r}_{\text {prop }}, \mathfrak{G} \mathfrak{r}_{\text {tight }}$ and $\mathfrak{G r}_{\text {bicor }}$, respectively.

Next we show some properties of the bicategory of bicorrespondences. This bicategory consists of arrows with no predefined direction. We make this precise in the following section.

\section{The symmetric bicategory $\mathfrak{G r}_{\text {bicor }}$}

The bicategory $\mathfrak{G r}_{\text {bicor }}$ carries a natural involution making it a symmetric bicategory. It is essential here to distinguish between the notions of commutativity and symmetry. The notion of commutativity works well when the category under 
2 Groupoid bicategories and the bicategory of $\mathrm{C}^{*}$-correspondences

consideration has a single object; it becomes less useful when applied to other categories. As a consequence, the notion of a symmetric bicategory, when restricted to single object categories, is more general than symmetric monoidal categories

Recall that we are mainly interested in bicategories where all 2-arrows are invertible. This makes the definition of a symmetric bicategory a little simpler.

Definition 2.31 (compare [30, Definition 16.2.1]). An involution on a category $\mathcal{C}$ is a functor $(*, \omega): \mathcal{C} \rightarrow \mathcal{C}^{\text {op }}$ such that

(1) the map $*: \mathcal{C}^{0} \rightarrow \mathcal{C}^{0}$ is bijective with $\left(A^{*}\right)^{*}=A$ for all $A \in \mathcal{C}^{0}$,

(2) the functor $*: \mathcal{C}(A, B) \rightarrow \mathcal{C}\left(B^{*}, A^{*}\right)=\mathcal{C}^{\text {op }}\left(A^{*}, B^{*}\right)$ gives an equivalence of categories for all objects $A$ and $B$ in $\mathcal{C}$; the natural isomorphisms of the equivalence are given by 2 -arrows $\xi: f \rightarrow f^{* *}$,

(3) the following diagram commutes

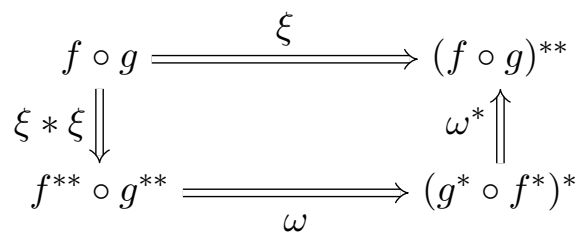

for all composable arrows $f$ and $g \in \mathcal{C}^{1}$.

A symmetric bicategory is a bicategory with an involution. An object $A \in \mathcal{C}$ is called commutative if $A^{*}=A$.

Proposition 2.32. The bicategory $\mathfrak{G r}_{\mathrm{bicor}}$ is symmetric.

Proof. We define an involution $(*, \omega)$ on $\mathfrak{G r}_{\text {bicor }}$ as follows. Let $\mathcal{G}^{*}:=\mathcal{G}^{\text {op }}$ for all groupoids $\mathcal{G}$. For a bicorrespondence $\mathcal{X}: \mathcal{H} \rightarrow \mathcal{G}$ we define $\mathcal{X}^{*}:=\mathcal{X}$ as a topological space. The left action of $\mathcal{G}^{*}$ on $\mathcal{X}^{*}$ is given by the anchor map $r_{\mathcal{X}^{*}}:=s \mathcal{X}$ and the multiplication map

$$
\mathcal{G}^{*} \times{ }_{s, \mathcal{G}^{0}, s_{\mathcal{X}}} \mathcal{X}^{*} \rightarrow \mathcal{X}^{*}, \quad(g, x) \mapsto x \cdot g .
$$

Similarly, $\mathcal{X}^{*}$ is a right $\mathcal{H}^{*}$-space. The left and right actions commute and they are both free and proper, since $\mathcal{X}$ is a bicorrespondence. This makes $\mathcal{X}^{*}: \mathcal{G}^{*} \rightarrow \mathcal{H}^{*}$ a bicorrespondence. For an isomorphism $\gamma: \mathcal{X}_{1} \rightarrow \mathcal{X}_{2}$, we define $\gamma^{*}:=\gamma$. This defines functors $*: \mathfrak{G r}(\mathcal{G}, \mathcal{H}) \rightarrow \mathfrak{G r}\left(\mathcal{H}^{*}, \mathcal{G}^{*}\right)$ for all groupoids $\mathcal{G}$ and $\mathcal{H}$. Let $\mathcal{X}: \mathcal{G} \rightarrow \mathcal{H}$ and $\mathcal{Y}: \mathcal{H} \rightarrow \mathcal{K}$ be correspondences. we define

$$
w_{\mathcal{X}, \mathcal{Y}}: \mathcal{Y}^{*} \circ_{\mathcal{H}} \mathcal{X}^{*} \rightarrow\left(\mathcal{X} \circ_{\mathcal{H}} \mathcal{Y}\right)^{*}, \quad[y, x] \mapsto[x, y] .
$$


This is an isomorphism of correspondences. In addition, for all $\mathcal{X} \in \mathfrak{G r}^{1}$ we define

$$
\xi=\mathrm{id}:\left(\mathcal{X}^{*}\right)^{*} \rightarrow \mathcal{X}
$$

We leave it to the reader to check that $(*, w)$ is a functor $\mathfrak{G} \mathfrak{r} \rightarrow \mathfrak{G r}^{\text {op }}$ and the functors $*: \mathfrak{G} \mathfrak{r}(\mathcal{G}, \mathcal{H}) \rightarrow \mathfrak{G} \mathfrak{r}(\mathcal{H}, \mathcal{G})$ are isomorphisms of categories. Moreover, Condition (2.16) is satisfied since $w^{*} \circ w=\mathrm{id}$.

\subsection{The functor to $\mathfrak{C o r r}$}

We are going to construct a covariant functor from the bicategory $\mathfrak{G r}$ to the correspondence bicategory of $\mathrm{C}^{*}$-algebras, which is studied in [11]. A similar functor of bicategories is constructed in [18]. The construction is very close to the construction of Morita-Rieffel equivalences between groupoid $\mathrm{C}^{*}$-algebras from equivalences of groupoids in [34], which is extended to the non-Hausdorff case in [41].

Let $\mathcal{G}$ be an étale groupoid. We call a function on $\mathcal{G}$ quasi-continuous if it is a finite linear combination of compactly supported functions $U \rightarrow \mathbb{C}$ for Hausdorff, open subsets $U$ of $\mathcal{G}$, where we extend a function on $U$ by 0 outside $U$ to a function on $\mathcal{G}$. If $\mathcal{G}$ is non-Hausdorff, these functions are not continuous. Let $\mathfrak{S}(\mathcal{G})$ be the $\mathbb{C}$-vector space of quasi-continuous functions on $\mathcal{G}$.

We define an involution and a convolution on $\mathfrak{S}(\mathcal{G})$ as in $[22]$ :

$$
\begin{aligned}
\xi \star \eta(g) & =\sum_{h: r(h)=r(g)} \xi(h) \eta\left(h^{-1} g\right), \\
\xi^{*}(g) & =\overline{\xi\left(g^{-1}\right)}
\end{aligned}
$$

for $\xi, \eta \in \mathfrak{S}(\mathcal{G})$.

To make $\mathfrak{S}(\mathcal{G})$ a normed ${ }^{*}$-algebra we define a norm as follows:

$$
\begin{aligned}
\|f\|_{I, r} & :=\sup _{u \in \mathcal{G}^{0}} \sum_{z: r(z)=u}|f(z)|, \\
\|f\|_{I, s} & :=\sup _{u \in \mathcal{G}^{0}} \sum_{z: r(z)=u}\left|f\left(z^{-1}\right)\right| .
\end{aligned}
$$

Then

$$
\|f\|_{I}:=\max \left\{\|f\|_{I, r},\|f\|_{I, s}\right\} .
$$

We need the following fact about $\mathrm{C}^{*}$-algebras of étale groupoids. 
2 Groupoid bicategories and the bicategory of $\mathrm{C}^{*}$-correspondences

Lemma 2.33 ([38, p. 47]). Let $\mathcal{G}$ be an étale groupoid. Then $\mathfrak{S}(\mathcal{G})$ has a bounded approximate identity with respect to the I-norm.

Proof. Since $\mathcal{G}^{0}$ is open in $\mathcal{G}$ and Hausdorff, Urysohn's lemma gives a sequence $\left\{\xi_{n}\right\}$ in $\mathrm{C}_{\mathrm{c}}\left(\mathcal{G}^{0}\right) \subset \mathfrak{S}(\mathcal{G})$ such that $0 \leq \xi_{n} \leq 1$ for every $n \in \mathbb{N}$ and such that $\mathcal{G}^{0}=$ $\bigcup_{n \in \mathbb{N}} U_{n}$, where $U_{n}$ is the interior of the set $\left\{u \in \mathcal{G}^{0} \mid \xi_{n}(u)=1\right\}$ and $U_{n} \subset U_{n+1}$.

$$
\left\|\xi_{n}\right\|_{I}=\left\|\xi_{n}\right\|_{I, r}=\left\|\xi_{n}\right\|_{I, s}=\sup _{u \in \mathcal{G}^{0}}\left|\xi_{n}(u)\right|=1 .
$$

It follows that the sequence $\left\{\xi_{n}\right\}$ is bounded with respect to the I-norm. If $\xi \in \mathfrak{S}(\mathcal{G})$ then $n \in \mathbb{N}$ we have

$$
\xi \star \xi_{n}(g)=\sum_{r(k)=s(g)} \xi(g k) \xi_{n}\left(k^{-1}\right)
$$

for all $g \in \mathcal{G}$ and $n \in \mathbb{N}$. Since $\xi_{n} \in \mathfrak{S}\left(\mathcal{G}^{0}\right)$, the last formula can be rewritten as

$$
\xi \star \xi_{n}(g)=\xi(g s(g)) \xi_{n}(s(g))=\xi(g) \xi_{n}(s(g)) .
$$

We may assume without loss of generality that the support of $f$ is contained in a compact set $C \subset \mathcal{G}$. Then we can pick $n \in \mathbb{N}$ large enough such that $s(C) \subset U_{n}$, and $f \star f_{n}=f$ for such $n$. Similarly, $f_{n} \star f=f$, so $\left\{f_{n}\right\}$ is an approximate identity for $\mathfrak{S}(\mathcal{G})$.

Definition 2.34. A correspondence from a $\mathrm{C}^{*}$-algebra $A$ to a $\mathrm{C}^{*}$-algebra $B$ is a Hilbert $B$-module $\mathcal{F}$ with a nondegenerate left action of $A$ by adjointable operators. A correspondence is proper if $A$ acts by compact operators. An isomorphism between two correspondences from $A$ to $B$ is a unitary operator on the underlying Hilbert $B$-modules that intertwines the left actions of $A$.

There is a bicategory $\mathfrak{C o r r}$ that has $\mathrm{C}^{*}$-algebras as objects, correspondences as arrows, isomorphisms of correspondences as 2-arrows, and the interior tensor product as composition of arrows, see [11].

Let $\mathcal{G}$ and $\mathcal{H}$ be groupoids and let $\mathcal{X}: \mathcal{H} \rightarrow \mathcal{G}$ be a correspondence from $\mathcal{G}$ to $\mathcal{H}$. We are going to construct a $\mathrm{C}^{*}$-correspondence $\mathrm{C}^{*}(\mathcal{X}): \mathrm{C}^{*}(\mathcal{H}) \rightarrow \mathrm{C}^{*}(\mathcal{G})$ as follows. We equip $\mathfrak{S}(\mathcal{X})$ with the right $\mathfrak{S}(\mathcal{G})$-action

$$
\xi \cdot b(y):=\sum_{\gamma \in \mathcal{G}: r(\gamma)=s(y)} \xi(y \cdot \gamma) b\left(\gamma^{-1}\right)
$$

for $\xi \in \mathfrak{S}(\mathcal{X})$ and $y \in \mathcal{X}$, with the $\mathfrak{S}(\mathcal{G})$-valued inner product

$$
\langle\xi, \eta\rangle(g):=\sum_{y \in \mathcal{X}: r(g)=s(y)} \overline{\xi(y)} \eta(y \cdot g)
$$


for $\xi, \eta \in \mathfrak{S}(\mathcal{X})$ and $g \in \mathcal{G}$, and with the left $\mathfrak{S}(\mathcal{H})$-module structure

$$
\varphi(a)(\xi)(y):=\sum_{h \in \mathcal{H}: r(h)=r(y)} a(h) \xi\left(h^{-1} \cdot y\right)
$$

for $a \in \mathfrak{S}(\mathcal{H}), \xi \in \mathfrak{S}(\mathcal{X})$ and $y \in \mathcal{X}$.

If $\mathcal{X}$ were an equivalence of groupoids, we could also define a left inner product and complete $\mathcal{X}$ to a full Hilbert $\mathrm{C}^{*}(\mathcal{H}), \mathrm{C}^{*}(\mathcal{G})$-bimodule, see [41, Corollaire 5.4]; the statement in the Hausdorff case without coefficients in [34, Theorem 2.8] is slightly more transparent, and the proof in [35] is far more detailed. The main issue is to prove the positivity of the inner product, so as to get a Hilbert module completion $\mathrm{C}^{*}(\mathcal{X})$, and to prove that the left action on $\mathfrak{S}(\mathcal{X})$ extends to an action on $\mathrm{C}^{*}(\mathcal{X})$. If we only assume that $\mathcal{X}$ is a groupoid correspondence, then part of the proof still works in the same way and shows that $\mathrm{C}^{*}(\mathcal{X})$ is a correspondence from $\mathrm{C}^{*}(\mathcal{H})$ to $\mathrm{C}^{*}(\mathcal{G})$. If $\mathcal{G}, \mathcal{H}$ and $\mathcal{X}$ are Hausdorff, this is proved in [18], even for groupoids with Haar systems. Given this long list of nearby results, we allow ourselves to omit the proof of the following proposition:

Proposition 2.35. There is a unique completion of $\mathfrak{S}(\mathcal{X})$ to a Hilbert $\mathrm{C}^{*}(\mathcal{G})$ module $\mathrm{C}^{*}(\mathcal{X})$, and the left $\mathrm{C}^{*}(\mathcal{H})$-action on $\mathfrak{S}(\mathcal{X})$ extends to a nondegenerate ${ }^{*}$-homomorphism $\mathrm{C}^{*}(\mathcal{H}) \rightarrow \mathbb{B}(\mathfrak{S}(\mathcal{X}))$.

Thus $\mathrm{C}^{*}(\mathcal{X})$ is a $\mathrm{C}^{*}$-correspondence $\mathrm{C}^{*}(\mathcal{H}) \rightarrow \mathrm{C}^{*}(\mathcal{G})$.

Let $\mathcal{X} \cong \mathcal{X}^{\prime}: \mathcal{H} \rightarrow \mathcal{G}$ be an isomorphism of groupoid correspondences. Clearly, this induces an isomorphism of $\mathrm{C}^{*}$-correspondences

$$
\mathrm{C}^{*}(\mathcal{X}) \cong \mathrm{C}^{*}\left(\mathcal{X}^{\prime}\right)
$$

The identity groupoid correspondence $\mathcal{G}$ on a groupoid $\mathcal{G}$ is mapped to the identity $\mathrm{C}^{*}$-correspondence $\mathrm{C}^{*}(\mathcal{G})$ on the groupoid $\mathrm{C}^{*}$-algebra. If two correspondences $\mathcal{X}: \mathcal{H} \rightarrow \mathcal{G}$ and $\mathcal{Y}: \mathcal{G} \rightarrow \mathcal{K}$ are composable, then there is a canonical isomorphism

$$
\mathrm{C}^{*}(\mathcal{X} \circ \mathcal{Y}) \cong \mathrm{C}^{*}(\mathcal{X}) \otimes_{\mathrm{C}^{*}(\mathcal{G})} \mathrm{C}^{*}(\mathcal{Y})
$$

which is induced by the map

$$
\mu_{\mathcal{X}, \mathcal{Y}}: \mathfrak{S}(\mathcal{X}) \otimes_{\mathfrak{S}(\mathcal{G})} \mathfrak{S}(\mathcal{Y}) \rightarrow \mathfrak{S}(\mathcal{X} \circ \mathcal{Y}), \quad \mu(f \otimes g)([x, y]):=\sum f(x) g(y),
$$

where the sum runs over all representatives $(x, y) \in \mathcal{X} \times{ }_{s, \mathcal{G}^{0}, r} \mathcal{Y}$ of $[x, y] \in \mathcal{X} \circ \mathcal{Y}$. This map is a $\mathfrak{S}(H)-\mathfrak{S}(K)$-bimodule map, preserves the $\mathfrak{S}(K)$-valued inner product and has a dense range. Hence it extends to an isomorphism of correspondences as in Equation (2.21).

Proposition 2.36. The data above defines a covariant functor from the bicategory of groupoids $\mathfrak{G} \mathfrak{r}$ to the $\mathrm{C}^{*}$-correspondence bicategory $\mathfrak{C o r r}$. 
2 Groupoid bicategories and the bicategory of $\mathrm{C}^{*}$-correspondences

Proof. The conditions for a functor between bicategories are formulated, for instance, in [25]. They are routine to check here.

Remark 2.37. Let $\mathcal{X}: \mathcal{H} \rightarrow \mathcal{G}$ be a bicorrespondence. Similar arguments as above show that $\mathrm{C}^{*}(\mathcal{X})$ is a Hilbert $\mathrm{C}^{*}(\mathcal{H}), \mathrm{C}^{*}(\mathcal{G})$-bimodule in the sense of Watatani, see [19]. The left inner product is

$$
\langle\xi, \eta\rangle(h):=\sum_{y \in \mathcal{X}: r(h)=r(y)} \xi\left(g^{-1} \cdot y\right) \overline{\eta(y)}
$$

for $\xi, \eta \in \mathfrak{S}(\mathcal{X})$ and $h \in \mathcal{H}$.

The left and right inner products for a Hilbert bimodule associated with a bicorrespondence do not satisfy any compatibilty condition, therefore it does not give a Hilbert bimodule as in [11], for instance.

Now assume, in addition, that $r, s: \mathcal{X} \rightarrow \mathcal{G}^{0}$ are surjective. This implies that the Hilbert bimodule $\mathrm{C}^{*}(\mathcal{X})$ is full (as a left and right Hilbert module). Therefore, it is a bi-Hilbertian bimodule in the sense of Kajiwara-Pinzari-Watatani (see [19, Definition 2.3]). It follows that the functor $\mathcal{F}: \mathfrak{G r} \rightarrow \mathfrak{C o r r}$ restricts to a functor $\mathcal{F}: \mathfrak{G r}_{\text {bicor }} \rightarrow \mathfrak{C o r r}_{\text {Hilb }}$, where $\mathfrak{C o r r}_{\text {Hilb }}$ is the subbicategory of $\mathfrak{C o r r}$ of Hilbert bimodules in Watatani's sense. Moreover, the image under $\mathcal{F}$ of a bicorrespondence with both anchor maps being surjective is a bi-Hilbertian bimodule.

Finally, if the bicorrespondence $\mathcal{X}$ is a partial equivalence, then $\mathrm{C}^{*}(\mathcal{X})$ is a Hilbert bimodule in the usual sense, see [10].

Proposition 2.38. Let $\mathcal{G}, \mathcal{H}$ be groupoids and let $\mathcal{X}: \mathcal{G} \rightarrow \mathcal{H}$ be a correspondence. The $\mathrm{C}^{*}$-correspondence $\mathrm{C}^{*}(\mathcal{X})$ is proper if the groupoid correspondence $\mathcal{X}$ is proper.

Proof. Let $\mathcal{X}{ }_{{ }_{\mathcal{G}}} \mathcal{X}^{*}$ denote the orbit space of the action of $\mathcal{G}$ on the space $\mathcal{X} \times{ }_{s, \mathcal{G}^{0}, s} \mathcal{X}$ given by $(x, y) \cdot g:=(x g, y g)$ for all $(x, y) \in \mathcal{X} \times_{s, \mathcal{G}^{0}, s} \mathcal{X}$ and all $g \in \mathcal{G}$ with $r(g)=$ $s(x)$. Let $f \in \mathfrak{S}\left(\mathcal{X} \circ_{\mathcal{G}} \mathcal{X}^{*}\right)$ be a compactly supported function. We set

$$
\psi(f)(\zeta)(x):=\sum_{y \in \mathcal{X}: s(x)=s(y)} f(x, y) \zeta(y)
$$

for all $x \in \mathcal{X}$ and all $\zeta \in \mathfrak{S}(\mathcal{X})$. Let $\xi, \eta \in \mathfrak{S}(\mathcal{X})$. Then the function $\theta_{\eta, \xi} \in$ $\mathfrak{S}\left(\mathcal{X} \circ_{\mathcal{G}} \mathcal{X}_{q}\right)$ defined by

$$
\theta_{\eta, \xi}(x, y):=\sum_{g \in \mathcal{G}: r(g)=s(y)} \eta(x \cdot g) \cdot \overline{\xi(y \cdot g)}
$$


satisfies

$$
\begin{aligned}
\psi\left(\theta_{\eta, \xi}\right)(\zeta)(x) & =\sum_{y \in \mathcal{X}: s(x)=s(y)} \zeta(y) \sum_{g \in \mathcal{G}: r(g)=s(x)} \eta(x \cdot g) \cdot \overline{\xi(y \cdot g)} \\
& =\sum_{g \in \mathcal{G}: r(g)=s(x)} \eta(x \cdot g) \sum_{y \in \mathcal{X}: s(g)=s(y)} \overline{\xi(y)} \zeta\left(y \cdot g^{-1}\right) \\
& =\sum_{g \in \mathcal{G}: r(g)=s(x)} \eta(x \cdot g)\langle\xi, \zeta\rangle\left(g^{-1}\right) \\
& =\eta *\langle\xi, \zeta\rangle(x) .
\end{aligned}
$$

Therefore, $\psi\left(\theta_{\eta, \xi}\right)=|\eta\rangle\langle\xi| \in \mathbb{K}\left(\mathrm{C}^{*}(\mathcal{X})\right)$. Let $a \in \mathfrak{S}\left(\mathcal{H}^{1}\right)$. Then $a$ acts on $\mathfrak{S}(\mathcal{X})$ by

$$
\varphi(a) \cdot \xi(x)=\sum_{h \in \mathcal{G}: r(h)=r(x)} a(h) \xi\left(h^{-1} x\right) .
$$

The correspondence $\mathrm{C}^{*}(\mathcal{X})$ is proper if $\varphi(a)$ is a compact operator for all $a \in \mathfrak{S}(\mathcal{H})$. Since $\mathcal{H}$ is an étale groupoid, by Lemma 2.33, there is an approximate unit $\left\{a_{i}\right\}$ for $\mathrm{C}^{*}(\mathcal{H})$ such that $a_{i} \in \mathrm{C}_{0}\left(\mathcal{H}^{0}\right)$. It follows that $\mathrm{C}^{*}(\mathcal{X})$ is proper if and only if $\varphi\left(\mathrm{C}_{\mathrm{c}}\left(\mathcal{H}^{0}\right)\right) \subset \mathbb{K}\left(\mathrm{C}^{*}(\mathcal{X})\right)$. Let $a \in \mathrm{C}_{\mathrm{c}}\left(\mathcal{H}^{0}\right)$. We define

$$
f_{a}(x, y):= \begin{cases}a(r(y)) & \text { if } x=y \\ 0 & \text { otherwise. }\end{cases}
$$

Then

$$
\psi\left(f_{a}\right)(\xi)(x)=a(r(x)) \xi(x)=\varphi(a)(\xi)(x) .
$$

Hence $\varphi(a)$ is a compact operator if its integral kernel $f_{a}$ is compactly supported on the diagonal $D:=\left\{[x, x] \in \mathcal{X} \circ \mathcal{X}^{*}\right\}$. The diagonal $D$ is homeomorphic to the space $\mathcal{X} / \mathcal{G}$ via the map $[x] \mapsto[x, x]$. Furthermore, the map $r_{\mathcal{X}_{*}}: \mathcal{X} / \mathcal{G} \rightarrow \mathcal{H}^{0}$ is proper if and only if it induces a map $\mathrm{C}_{0}\left(\mathcal{H}^{0}\right) \rightarrow \mathrm{C}_{0}(\mathcal{X} / \mathcal{G})$. This concludes our proof.

Example 2.39. Let $\mathcal{G}$ and $\mathcal{H}$ be the same locally compact space, viewed as a groupoid. Then a groupoid correspondence $\mathcal{H} \rightarrow \mathcal{G}$ is the same as a topological graph [20] with vertex space $\mathcal{G}=\mathcal{H}$, that is, a space $\mathcal{X}$ with a continuous map $\mathcal{H} \rightarrow \mathcal{X}$ and a local homeomorphism $\mathcal{X} \rightarrow \mathcal{G}$ (see Example 2.22). The groupoid $\mathrm{C}^{*}$-algebra of $\mathcal{G}$ is the commutative $\mathrm{C}^{*}$-algebra of $\mathrm{C}_{0}$-functions on $\mathcal{G}$, and $\mathrm{C}^{*}(\mathcal{X})$ is the $\mathrm{C}^{*}$-correspondence associated to a topological graph as in [20]. Proposition 2.38 says that such a correspondence is proper if and only if the map $\mathcal{H} \rightarrow \mathcal{X}$ is proper, which is well-known (compare [33, Corollary 3.12]). The composition of groupoid correspondences in this case is the usual fibre product, and the multiplicativity of the map $\mathcal{X} \mapsto \mathrm{C}^{*}(\mathcal{X})$ is [1, Lemma 4.3], compare also [33, Lemmas 6.1-4]. 
2 Groupoid bicategories and the bicategory of $\mathrm{C}^{*}$-correspondences

\subsection{Diagrams and colimits}

\subsubsection{Diagrams of groupoid correspondences}

In this section, we define diagrams in the bicategory of groupoid correspondences, natural transformations between such diagrams and colimits. We finish this section by describing colimits of group actions in $\mathfrak{G r}$.

Let $\mathcal{C}$ be a category. We denote by $\mathfrak{G r}^{\mathcal{C}}$ the bicategory whose objects are functors $\mathcal{C} \rightarrow \mathfrak{G} \mathfrak{r}$, arrows are transformations between such functors, and 2-arrows are modifications (see [25]).

Functors were defined in Definition 2.2 and transformations between functors in Definition 2.4. The following propositions can be considered as the definitions of transformations, modifications and functors in $\mathfrak{G r}$. Their proofs are similar to the proofs in the $\mathrm{C}^{*}$-algebraic setting in [11].

Proposition 2.40. A functor $\mathcal{C} \rightarrow \mathfrak{G r}$ consists of

- locally compact groupoids $\mathcal{G}_{x}$ for all objects $x$ of $\mathcal{C}$;

- correspondences $\mathcal{X}_{g}: \mathcal{G}_{x} \rightarrow \mathcal{G}_{y}$ for all arrows $g: x \rightarrow y$ in $\mathcal{C}$;

- isomorphisms $\mu_{g, h}: \mathcal{X}_{h} \circ_{\mathcal{G}_{y}} \mathcal{X}_{g} \rightarrow \mathcal{X}_{g h}$ for all pairs of composable arrows $g: y \rightarrow z, h: x \rightarrow y$ in $\mathcal{C}$

such that

(1) $\mathcal{X}_{1_{x}}$ is the identity correspondence on $\mathcal{G}_{x}$ for all objects $x$ of $\mathcal{C}$;

(2) $\mu_{1_{y}, g}: \mathcal{X}_{g} \circ_{\mathcal{G}_{y}} \mathcal{G}_{y} \rightarrow \mathcal{X}_{g}$ and $\mu_{g, 1_{x}}: \mathcal{G}_{x} \circ_{\mathcal{G}_{x}} \mathcal{X}_{g} \rightarrow \mathcal{X}_{g}$ are the canonical isomorphisms for all arrows $g: x \rightarrow y$ in $\mathcal{C}$;

(3) for all composable arrows $g_{01}: x_{0} \rightarrow x_{1}, g_{12}: x_{1} \rightarrow x_{2}, g_{23}: x_{2} \rightarrow x_{3}$, the following diagram commutes:

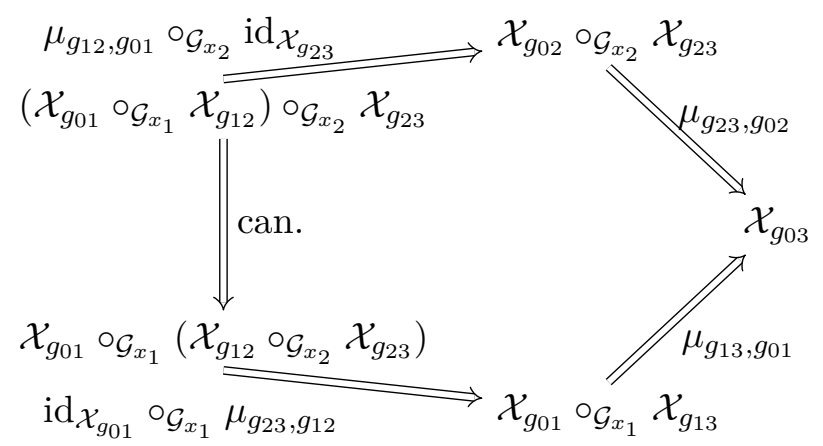

Here $g_{02}:=g_{12} \circ g_{01}, g_{13}:=g_{23} \circ g_{12}$, and $g_{03}:=g_{23} \circ g_{12} \circ g_{01}$. 
The diagram (2.31) commutes automatically if one of the arrows $g_{01}, g_{12}$ or $g_{23}$ is an identity arrow.

Proposition 2.41. Let $\left(\mathcal{G}_{x}^{0}, \mathcal{X}_{g}^{0}, \mu_{g, h}^{0}\right)$ and $\left(\mathcal{G}_{x}^{1}, \mathcal{X}_{g}^{1}, \mu_{g, h}^{1}\right)$ be two functors from $\mathcal{C}$ to $\mathfrak{G r}$. A transformation between them consists of

- proper correspondences $\gamma_{x}$ from $\mathcal{G}_{x}^{0}$ to $\mathcal{G}_{x}^{1}$ for all objects $x$ of $\mathcal{C}$;

- isomorphisms $V_{g}: \gamma_{x}{ }^{\circ} \mathcal{G}_{x}^{1} \mathcal{X}_{g}^{1} \rightarrow \mathcal{X}_{g}^{0}{ }^{\circ} \mathcal{G}_{y}^{0} \gamma_{y}$ for all arrows $g: x \rightarrow y$ in $\mathcal{C}$;

such that

(1) $V_{1_{x}}: \gamma_{x}{ }^{\circ}{ }_{\mathcal{G}_{x}^{1}} \mathcal{G}_{x}^{1} \rightarrow \mathcal{G}_{x}^{0}{ }^{\circ}{ }_{\mathcal{G}_{x}^{0}} \gamma_{x}$ is the canonical isomorphism for each object $x$ in $\mathcal{C}$;

(2) for each pair of composable arrows $g: y \rightarrow z, h: x \rightarrow y$ in $\mathcal{C}$, the following diagram commutes:

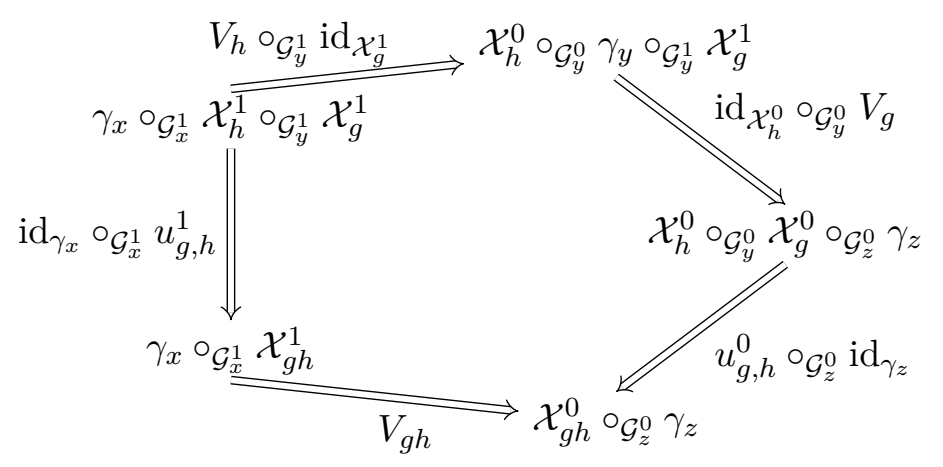

The diagram (2.27) commutes automatically if $g$ or $h$ is an identity arrow.

Proposition 2.42. Let $\left(\mathcal{G}_{x}^{0}, \mathcal{X}_{g}^{0}, \mu_{g, h}^{0}\right)$ and $\left(\mathcal{G}_{x}^{1}, \mathcal{X}_{g}^{1}, \mu_{g, h}^{1}\right)$ be functors from $\mathcal{C}$ to $\mathfrak{G r}$ and let $\left(\gamma_{x}^{1}, V_{g}^{1}\right)$ and $\left(\gamma_{x}^{2}, V_{g}^{2}\right)$ be transformations between them. A modification from $\left(\gamma_{x}^{1}, V_{g}^{1}\right)$ to $\left(\gamma_{x}^{2}, V_{g}^{2}\right)$ consists of isomorphisms of proper correspondences $W_{x}: \gamma_{x}^{1} \rightarrow \gamma_{x}^{2}$ for each object $x$ in $\mathcal{C}$ such that the diagrams

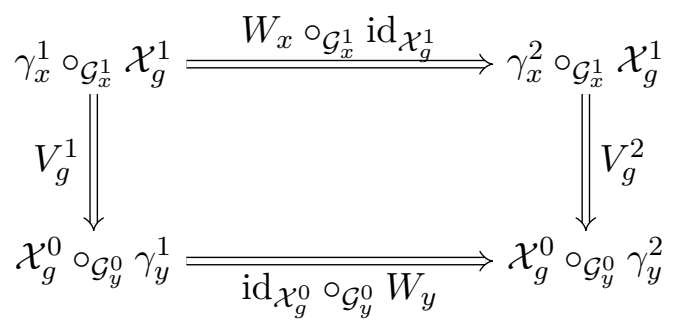

commute for all arrows $g: x \rightarrow y$ in $\mathcal{C}$. This diagram commutes automatically if $g$ is an identity arrow. 
2 Groupoid bicategories and the bicategory of $\mathrm{C}^{*}$-correspondences

The composition of transformations is defined as follows. Describe functors $\mathcal{C} \rightarrow$ $\mathfrak{G r}$ by $\left(\mathcal{G}_{x}^{0}, \mathcal{X}_{g}^{0}, \mu_{g, h}^{0}\right),\left(\mathcal{G}_{x}^{1}, \mathcal{X}_{g}^{1}, \mu_{g, h}^{1}\right)$ and $\left(\mathcal{G}_{x}^{2}, \mathcal{X}_{g}^{2}, \mu_{g, h}^{2}\right)$, and transformations between them by $\left(\gamma_{x}^{01}, V_{g}^{01}\right)$ and $\left(\gamma_{x}^{12}, V_{g}^{12}\right)$ as above. The composite transformation is defined by $\gamma_{x}^{02}:=\gamma_{x}^{01} \circ_{\mathcal{G}_{x}^{1}} \gamma_{x}^{12}$ for objects $x$ of $\mathcal{C}$ and

$$
\begin{aligned}
& V_{g}^{02}: \gamma_{x}^{02} \circ_{\mathcal{G}_{x}^{2}} \mathcal{X}_{g}^{2}=\gamma_{x}^{01} \circ_{\mathcal{G}_{x}^{1}} \gamma_{x}^{12} \circ_{\mathcal{G}_{x}^{2}} \mathcal{X}_{g}^{2} \stackrel{\mathrm{id}_{\gamma_{x}^{01}} \circ_{\mathcal{G}_{x}} V_{g}^{12}}{\longrightarrow} \gamma_{x}^{01}{ }^{\circ}{ }_{\mathcal{G}_{x}^{1}} \mathcal{X}_{g}^{1} \circ_{\mathcal{G}_{y}^{2}} \gamma_{y}^{12} \\
& \stackrel{V_{g}^{01}{ }^{\mathcal{G}_{y}}{ }^{2 \mathrm{id}} \gamma_{y}^{12}}{\longrightarrow} \mathcal{X}_{g}^{0} \circ_{\mathcal{G}_{y}^{0}} \gamma_{y}^{01} \circ_{\mathcal{G}_{y}^{1}} \gamma_{y}^{12}=\mathcal{X}_{g}^{0} \circ_{\mathcal{G}_{y}^{0}} \gamma_{y}^{02}
\end{aligned}
$$

for all arrows $g: x \rightarrow y$ in $\mathcal{C}$. These $\left(\gamma_{x}^{02}, V_{g}^{02}\right)$ indeed form a transformation.

Let $\mathcal{C}$ be a category and let $\left(\mathcal{G}_{x}, \mathcal{X}_{g}, \mu_{g, h}\right)$ describe a functor $\mathcal{C} \rightarrow \mathfrak{G r}$. To describe the colimit of this diagram, we only need transformations from $\left(\mathcal{G}_{x}, \mathcal{X}_{g}, \mu_{g, h}\right)$ to constant functors const ${ }_{D}: \mathcal{C} \rightarrow \mathfrak{G r}$, and modifications among such transformations. The following definition is a special case of Definition 2.3.

Definition 2.43. Let $\mathcal{D}$ be a locally compact groupoid. The constant functor const $_{\mathcal{D}}: \mathcal{C} \rightarrow \mathfrak{G r}$ maps all objects $x$ of $\mathcal{C}$ to $\mathcal{D}$, all arrows $g$ in $\mathcal{C}$ to the identity correspondence on $\mathcal{D}$, and all pairs $g, h$ to the canonical isomorphism $\mathcal{D} \circ_{\mathcal{D}} \mathcal{D} \rightarrow \mathcal{D}$.

By Definition 2.5, a transformation from the functor given by $\left(\mathcal{G}_{x}, \mathcal{X}_{g}, \mu_{g, h}\right)$ to const $_{\mathcal{D}}$ is given by correspondences $\gamma_{x}$ from $\mathcal{G}_{x}$ to $\mathcal{D}$ for all objects $x$ of $\mathcal{C}$ and isomorphisms of correspondences

$$
V_{g}: \gamma_{x} \rightarrow \mathcal{X}_{g} \circ_{\mathcal{G}_{y}} \gamma_{y} \quad \text { for all arrows } g: x \rightarrow y \text { in } \mathcal{C},
$$

such that $V_{1_{x}}$ is the canonical isomorphism for all objects $x$ and the diagrams

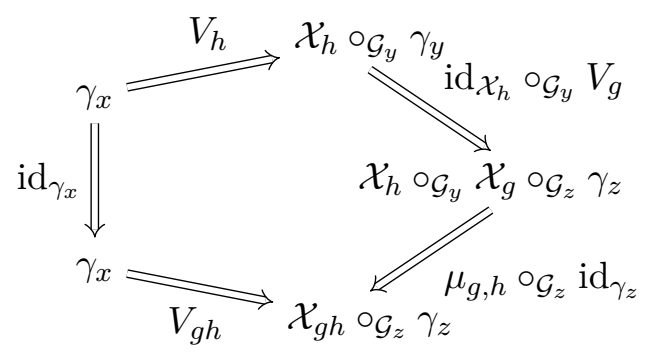

for composable arrows $g: y \rightarrow z, h: x \rightarrow y$ in $\mathcal{C}$ commute. This diagram commutes automatically if $g$ or $h$ is an identity arrow.

If $\left(\gamma_{x}^{1}, V_{g}^{1}\right)$ and $\left(\gamma_{x}^{2}, V_{g}^{2}\right)$ are two such transformations, then a modification between them is given by isomorphisms of correspondences

$$
W_{x}: \gamma_{x}^{1} \rightarrow \gamma_{x}^{2} \quad \text { for all objects } x \text { of } \mathcal{C},
$$


such that the diagrams

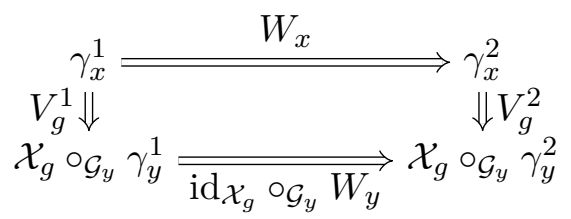

commute for all arrows $g: x \rightarrow y$ in $\mathcal{C}$, see Definition 2.6. This diagram commutes automatically if $g$ is an identity arrow.

The colimit for a functor $F: \mathcal{C} \rightarrow \mathfrak{G r}$ is, by definition, a groupoid $\mathcal{H}$ such that the groupoid of arrows $\mathcal{H} \rightarrow \mathcal{D}$ and 2-arrows between them is naturally equivalent to the groupoid of transformations $F \rightarrow$ const $_{\mathcal{D}}$ and the modifications between them for each locally compact groupoid $\mathcal{D}$.

\subsubsection{Diagrams of $\mathrm{C}^{*}$-correspondences}

In this section, we describe diagrams in Corr, transformation between such diagrams and modification between transformations. Our reference for this is [11, §4], where the same is worked out for the bicategory of $\mathrm{C}^{*}$-algebras with nondegenerate ${ }^{*}$-homomorphisms as arrows and unitary intertwiners as 2 -arrows.

Proposition 2.44. A functor $\mathcal{C} \rightarrow \mathfrak{C o r r}$ consists of

- $\mathrm{C}^{*}$-algebras $A_{x}$ for all objects $x$ of $\mathcal{C}$;

- correspondences $\mathcal{E}_{g}: A_{x} \rightarrow A_{y}$ for all arrows $g: x \rightarrow y$ in $\mathcal{C}$;

- isomorphisms of correspondences $\mu_{g, h}: \mathcal{E}_{h} \otimes_{A_{y}} \mathcal{E}_{g} \rightarrow \mathcal{E}_{g h}$ for all pairs of composable arrows $g: y \rightarrow z, h: x \rightarrow y$ in $\mathcal{C}$;

such that

(1) $\mathcal{E}_{1_{x}}$ is the identity correspondence on $A_{x}$ for all objects $x$ of $\mathcal{C}$;

(2) $\mu_{1_{y}, g}: \mathcal{E}_{g} \otimes_{A_{y}} A_{y} \rightarrow \mathcal{E}_{g}$ and $\mu_{g, 1_{x}}: A_{x} \otimes_{A_{x}} \mathcal{E}_{g} \rightarrow \mathcal{E}_{g}$ are the canonical isomorphisms for all arrows $g: x \rightarrow y$ in $\mathcal{C}$;

(3) for all composable arrows $g_{01}: x_{0} \rightarrow x_{1}, g_{12}: x_{1} \rightarrow x_{2}, g_{23}: x_{2} \rightarrow x_{3}$, the following diagram commutes:

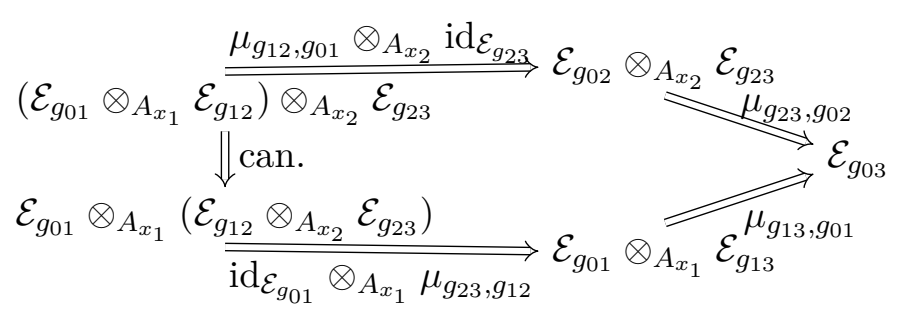


2 Groupoid bicategories and the bicategory of $\mathrm{C}^{*}$-correspondences

Here $g_{02}:=g_{12} \circ g_{01}, g_{13}:=g_{23} \circ g_{12}$, and $g_{03}:=g_{23} \circ g_{12} \circ g_{01}$.

The diagram (2.31) commutes automatically if one of the arrows $g_{01}, g_{12}$ or $g_{23}$ is an identity arrow.

Proposition 2.45. Let $\left(A_{x}^{0}, \mathcal{E}_{g}^{0}, \mu_{g, h}^{0}\right)$ and $\left(A_{x}^{1}, \mathcal{E}_{g}^{1}, \mu_{g, h}^{1}\right)$ be two functors from $\mathcal{C}$ to $\mathfrak{C o r r}$. A transformation between them consists of

- correspondences $\gamma_{x}$ from $A_{x}^{0}$ to $A_{x}^{1}$ for all objects $x$ of $\mathcal{C}$;

- isomorphisms of correspondences $V_{g}: \gamma_{x} \otimes_{A_{x}^{1}} \mathcal{E}_{g}^{1} \rightarrow \mathcal{E}_{g}^{0} \otimes_{A_{y}^{0}} \gamma_{y}$ for all arrows $g: x \rightarrow y$ in $\mathcal{C}$;

such that

(1) $V_{1_{x}}: \gamma_{x} \otimes_{A_{x}^{1}} A_{x}^{1} \rightarrow A_{x}^{0} \otimes_{A_{x}^{0}} \gamma_{x}$ is the canonical isomorphism for each object $x$ in $\mathcal{C}$;

(2) for each pair of composable arrows $g: y \rightarrow z, h: x \rightarrow y$ in $\mathcal{C}$, the following diagram commutes:

$$
\begin{aligned}
& V_{h} \otimes_{A_{y}^{1}} \stackrel{\operatorname{id}_{\mathcal{E}_{g}^{1}}}{\longrightarrow} \mathcal{E}_{h}^{0} \otimes_{A_{y}^{0}} \gamma_{y} \otimes_{A_{y}^{1}} \mathcal{E}_{g}^{1} \\
& \gamma_{x} \otimes_{A_{x}^{1}} \overline{\mathcal{E}_{h}^{1 \otimes_{A_{y}^{1}}} \mathcal{E}_{g}^{1}} \quad \operatorname{ld}_{\mathcal{E}_{h}^{0}} \otimes_{A_{y}^{0}} V_{g} \\
& \operatorname{id}_{\gamma_{x}} \otimes_{A_{x}^{1}} u_{g, h}^{1} \Downarrow \quad \mathcal{E}_{h}^{0} \otimes_{A_{y}^{0}} \mathcal{E}_{g}^{0} \otimes_{A_{z}^{0}} \gamma_{z} \\
& \gamma_{x} \otimes_{A_{x}^{1} \mathcal{E}_{g h}^{1}}^{\stackrel{V_{g h}}{\longrightarrow}} \mathcal{E}_{g h}^{0} \otimes_{A_{z}^{0}} \gamma_{z}^{0}{ }_{g, h}^{0} \otimes_{A_{z}^{0}} \operatorname{id}_{\gamma_{z}}
\end{aligned}
$$

The diagram (2.32) commutes automatically if $g$ or $h$ is an identity arrow.

Proposition 2.46. Let $\left(A_{x}^{0}, \mathcal{E}_{g}^{0}, \mu_{g, h}^{0}\right)$ and $\left(A_{x}^{1}, \mathcal{E}_{g}^{1}, \mu_{g, h}^{1}\right)$ be functors from $\mathcal{C}$ to $\mathfrak{C o r r}$ and let $\left(\gamma_{x}^{1}, V_{g}^{1}\right)$ and $\left(\gamma_{x}^{2}, V_{g}^{2}\right)$ be transformations between them. A modification from $\left(\gamma_{x}^{1}, V_{g}^{1}\right)$ to $\left(\gamma_{x}^{2}, V_{g}^{2}\right)$ consists of isomorphisms of correspondences $W_{x}: \gamma_{x}^{1} \rightarrow \gamma_{x}^{2}$ for all objects $x$ in $\mathcal{C}$ such that the diagrams

$$
\begin{gathered}
\gamma_{x}^{1} \otimes_{A_{x}^{1}} \mathcal{E}_{g}^{1} \stackrel{W_{x} \otimes_{A_{x}^{1}} \mathrm{id}_{\mathcal{E}_{g}^{1}}}{=} \gamma_{x}^{2} \otimes_{A_{x}^{1}} \mathcal{E}_{g}^{1} \\
V_{g}^{1 \Downarrow} \\
\mathcal{E}_{g}^{0} \otimes_{A_{y}^{0}} \gamma_{y}^{1} \underset{\overline{\mathrm{id}}_{\mathcal{E}_{g}^{0} \otimes_{A_{y}^{0}} W_{y}}}{\mathcal{E}_{g}^{0} \otimes_{A_{y}^{0}} \gamma_{y}^{2}}
\end{gathered}
$$

commute for all arrows $g: x \rightarrow y$ in $\mathcal{C}$. This diagram commutes automatically if $g$ is an identity arrow. 


\subsubsection{Examples}

\section{Group actions by homeomorphisms}

Lemma 2.47. Let $X$ be a locally compact space and let $G$ be a discrete group. There is an equivalence between $G$-actions on $X$ by homeomorphisms and morphisms $G \rightarrow \mathfrak{G r}$ which map the unique object in $G$ to $X$.

Proof. First, let $\alpha: G \rightarrow \operatorname{Homeo}(X)$ be a group action. That is, $\alpha_{g}:=\alpha(g)$ are homeomorphisms on $X$ such that $\alpha_{g} \alpha_{h}=\alpha_{g h}$ for all $g, h \in G$. Let $X_{g}$ be the correspondence given by $X_{g}:=X$ as a topological space, with anchor maps $r_{g}, s_{g}: X_{g} \rightarrow X$ given by

$$
r_{g}(x):=x, \quad s_{g}(x):=\alpha_{g}(x) .
$$

for all $x \in X$. To define a functor $G \rightarrow \mathfrak{G r}$ we still need to define the multiplication isomorphisms; these are given by

$$
\mu_{g, h}^{\alpha}: X_{g} \times_{s_{g}, X, r_{h}} X_{h} \stackrel{\sim}{\rightarrow} X_{h g}, \quad\left(x, \alpha_{g}(x)\right) \mapsto x .
$$

It is routine to check that $\left(X_{g}, \mu_{g, h}^{\alpha}\right)$ is indeed a functor $G \rightarrow \mathfrak{G r}$.

Conversely, let $\left(\mathcal{X}_{g}, \mu_{g, h}^{\alpha}\right)$ be a functor $G \rightarrow \mathfrak{G r}$ with $\mathcal{X}_{1}=X$. That is $\mathcal{X}_{g}: X \rightarrow X$ are correspondences and $\mu_{g, h}: \mathcal{X}_{g} \circ_{X} \mathcal{X}_{h} \stackrel{\sim}{\rightarrow} \mathcal{X}_{h g}$ are isomorphisms of correspondences. The correspondence $\mathcal{X}_{g}$ is an equivalence for each $g \in G$. By Theorem 2.30, it is a Morita equivalence over the space $X$. This implies that both $r: \mathcal{X}_{g} \rightarrow X$ and $s: \mathcal{X}_{g} \rightarrow X$ are homeomorphisms. Let

$$
\alpha_{g}:=s_{g} r_{g}^{-1}
$$

for all $g \in G$. Then

$$
G \rightarrow \operatorname{Homeo}(X), \quad g \mapsto \alpha_{g},
$$

defines an action of $G$ on the space $X$. Moreover, the maps $V_{g}:=s_{g}: \mathcal{X}_{g} \rightarrow X_{g}$, where $X_{g}: X \rightarrow X$ are the correspondences as in the first part of the proof, form an invertible transformation from the functor $\left(\mathcal{X}_{g}, \mu_{g, h}\right)$ to the functor associated to an action of $G$ on $X$ by homeomorphisms.

Lemma 2.48. Let $\alpha: G \rightarrow \operatorname{Homeo}(X)$ be a group action on a space $X$ by homeomorphism. The colimit of the action in $\mathfrak{G r}$ is the transformation groupoid $G \ltimes X$.

Proof. Let $\left(X_{g}, \mu_{g, h}^{\alpha}\right)$ be the functor associated to $\alpha: G \rightarrow \operatorname{Homeo}(X)$ as in Lemma 2.47. Let $\mathcal{D}$ be a groupoid and let $\left(\mathcal{Y}, V_{g}\right)$ be a transformation from $\left(X_{g}, \mu_{g, h}^{\alpha}\right)$ to const $_{\mathcal{D}}$. The group $G$ acts on $\mathcal{Y}$ by

$$
g \cdot y:=V_{g}\left(r_{\mathcal{Y}}(y), y\right) .
$$


Together with the $\mathcal{D}$-equivariant map $r_{\mathcal{Y}_{*}}: \mathcal{Y} / \mathcal{G} \rightarrow X$, the group action of $G$ on $\mathcal{Y}$ gives an action of the transformation groupoid $G \ltimes X$ on $\mathcal{Y}$ that commutes with the $\mathcal{D}$-action (see [31, Proposition 4.10]). This gives a correspondence $\mathcal{Y}: G \ltimes X \rightarrow$ $\mathcal{D}$. Conversely, let $\mathcal{Y}: G \ltimes X \rightarrow \mathcal{D}$ be a correspondence. By [31, Proposition 4.10] this is equivalent to an action of $G$ on $\mathcal{Y}$ with a $G$-map $r_{\mathcal{Y}}: \mathcal{Y} \rightarrow X$. It follows that $\mathcal{Y}: X \rightarrow \mathcal{D}$ is a correspondence using the anchor map $r_{\mathcal{Y}}$. We define

$$
V_{g}\left(r_{\mathcal{Y}}(y), y\right):=g \cdot y
$$

for all $g \in G$ and all $y \in \mathcal{Y}$. Then $V_{g}: X \times_{\mathrm{id}, X, r_{\mathcal{Y}}} \mathcal{Y} \rightarrow \mathcal{Y}$ is an isomorphism of correspondences and $\left(\mathcal{Y}, V_{g}\right)$ satisfies the coherence condition in (2.29) and therefore gives a transformation from $\left(X_{g}, \mu_{g, h}^{\alpha}\right)$ to const $_{\mathcal{D}}$. The above two constructions are inverse to each other and natural in the formal sense. So they show that $G \rtimes X$ is the colimit. 


\section{Actions of Ore monoids}

In this chapter, we study Ore monoid actions in the bicategory of groupoid correspondences. The most basic example of an Ore monoid is the monoid of natural numbers $\mathbb{N}$. An action of $\mathbb{N}$ in $\mathfrak{G} r$ by proper correspondences is equivalent to a single correspondence $\mathcal{X}: \mathcal{G} \rightarrow \mathcal{G}$ for a groupoid $\mathcal{G}$. To associate a $\mathrm{C}^{*}$-algebra to the action given by $\mathcal{X}$, we may first use the functor $\mathfrak{G r} \rightarrow \mathfrak{C o r r}$ to obtain a $\mathrm{C}^{*}$-correspondence $\mathcal{E}:=\mathrm{C}^{*}(\mathcal{X}): \mathrm{C}^{*}(\mathcal{G}) \rightarrow \mathrm{C}^{*}(\mathcal{G})$ and then take the CuntzPimsner algebra $\mathcal{O}_{\mathcal{E}}$.

Now we know from [2] that $\mathcal{O}_{\mathcal{E}}$ is the colimit of the action given by $\mathcal{E}$ in the correspondence bicategory $\mathfrak{C o r r}$. We will show that the action given by $\mathcal{X}$ also has a colimit groupoid $\mathcal{H}$ in $\mathfrak{G r}$. Theorem 3.36 shows that $\mathcal{H}$ is a groupoid model of the Cuntz-Pimsner algebra $\mathcal{O}_{\mathcal{E}}$, that is, $\mathcal{O}_{\mathcal{E}} \cong \mathrm{C}^{*}(\mathcal{H})$.

Our construction of the groupoid colimit of an action of an Ore monoid $P$ by correspondence has two steps. We first show that an Ore monoid action by tight correspondences always has a colimit. This is done in Section 3.2. In Section 3.3 we show how to approximate an Ore monoid action by correspondences through an action by tight correspondences in a way that does not change the colimit, see Theorem 3.30.

\subsection{Ore monoids}

In this section, we introduce Ore monoids and their actions in the bicategory of groupoid correspondences $\mathfrak{G} \mathfrak{r}$.

Definition 3.1 ([27, Section IX.1]). A category $\mathcal{C}$ is filtered if it is nonempty and

(1) for any two objects $x, y \in \mathcal{C}_{0}$, there are $z \in \mathcal{C}_{0}, g \in \mathcal{C}(x, z)$ and $h \in \mathcal{C}(y, z)$;

(2) for any two parallel arrows $g, h \in \mathcal{C}(x, y)$, there are $z \in \mathcal{C}_{0}$ and $k \in \mathcal{C}(y, z)$ with $k g=k h$.

Let $P$ be a monoid and let $\mathcal{C}_{P}$ be the category with object set $P$ and arrow set $P \times P$, where $(p, q)$ is an arrow from $p$ to $p q$, and where $(p q, r) \cdot(p, q):=(p, q r)$ for all $p, q, r \in P$. The category $\mathcal{C}_{P}$ is filtered if and only if $P$ satisfies the following Ore conditions:

(Ore1) for all $x_{1}, x_{2} \in P$, there are $y_{1}, y_{2} \in P$ with $x_{1} y_{1}=x_{2} y_{2}$; 
(Ore2) if $x y_{1}=x y_{2}$ for $y_{1}, y_{2}, x \in P$, then there is $z \in P$ with $y_{1} z=y_{2} z$.

Definition 3.2. We call $P$ a right Ore monoid if the category $\mathcal{C}_{P}$ is filtered. We call $P$ a left Ore monoid if $P^{\text {op }}$ is a right Ore monoid.

Condition (Ore2) follows if $P$ has cancellation. Both hold if $P \subseteq G$ for a group $G$ with $P P^{-1}=G$.

Let $P$ be an Ore monoid. We may construct a group completion $G=G[P]$ of $P$ by taking equivalence classes of formal fractions $p q^{-1}:=(p, q) \in P \times P$, where $\left(p_{1}, q_{1}\right) \sim\left(p_{2}, q_{2}\right)$ if there are elements $a_{1}, a_{2} \in P$ with $\left(p_{1} a_{1}, q_{1} a_{1}\right)=$ $\left(p_{2} a_{2}, q_{2} a_{2}\right)$. The product of the elements $\left[p_{1}, q_{1}\right]$ and $\left[p_{2}, q_{2}\right]$ is given by

$$
\left[p_{1}, q_{1}\right]\left[p_{2}, q_{2}\right]:=\left[p_{1} t_{1}, q_{2} t_{2}\right]
$$

where $t_{1}, t_{2} \in P$ are such that $q_{1} t_{1}=p_{2} t_{2}$. The group $G$ is determined by the monoid $P$, and every monoid morphism $\varphi: P \rightarrow H$ for a group $H$ extends to a group homomorphism $\tilde{\varphi}: G \rightarrow H$. The group homomorphism is simply given by

$$
\tilde{\varphi}[p, q]=\varphi(p) \varphi(q)^{-1}
$$

for all $[p, q] \in G$.

Example 3.3. Commutative monoids are Ore monoids. Normal monoids are also Ore; here we say that a monoid $P$ is normal if $s P=P s$ for all $s \in P$. Non-abelian free monoids are examples of monoids which do not satisfy the Ore conditions.

An action of an Ore monoid $P$ in the bicategory $\mathfrak{G r}$ is a functor $F: P^{\mathrm{op}} \rightarrow \mathcal{G}$ (see Proposition 2.40). Here we think of an Ore monoid as a category with one object (the identity) and elements of the monoid as arrows; the product of two arrows $p$ and $q$ is $p q$.

We include the following definition for convenience.

Definition 3.4. Let $\mathcal{G}$ be a groupoid and let $P$ be an Ore monoid. An action of $P^{\mathrm{op}}$ on $\mathcal{G}$ by correspondences consists of the following data:

- correspondences $\mathcal{X}_{p}: \mathcal{G} \rightarrow \mathcal{G}$ for $p \in P \backslash\{1\}$;

- isomorphisms $\sigma_{p, q}: \mathcal{X}_{p q} \rightarrow \mathcal{X}_{p} \circ_{\mathcal{G}} \mathcal{X}_{q}$ for $p, q \in P \backslash\{1\}$.

We assume that $\mathcal{X}_{1}=\mathcal{G}$ is the identity correspondence and that $\sigma_{p, 1}$ and $\sigma_{1, q}$ are the canonical homeomorphisms $\mathcal{X}_{p} \cong \mathcal{X}_{p} \circ_{\mathcal{G}} \mathcal{G}$ and $\mathcal{X}_{q} \cong \mathcal{G} \circ_{\mathcal{G}} \mathcal{X}_{q}$ for $p, q \in P$. For an action of $P$, we also require the diagram

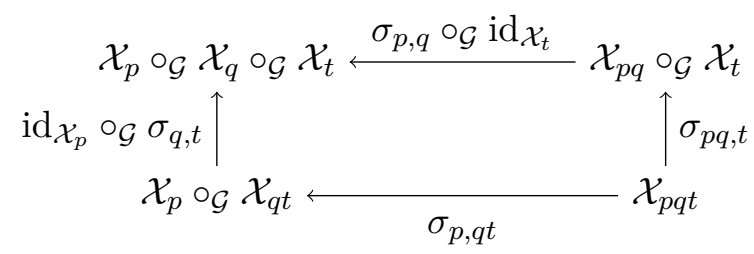


to commute for all $p, q, t \in P \backslash\{1\}$. This diagram commutes automatically if $p=1, q=1$ or $t=1$, so our assumption implies that it commutes for all $p, q, t \in P$.

\subsection{Actions of Ore monoids by tight correspondences}

In this section, we describe colimits of Ore monoid actions by tight correspondences.

Let $\mathcal{X}: \mathcal{G} \rightarrow \mathcal{G}$ be a tight correspondence. By Proposition 2.26, there is a local homeomorphism

$$
\mathcal{X} \times \mathcal{X} / \mathcal{G} X \rightarrow \mathcal{G}, \quad(x, y) \mapsto\langle x, y\rangle .
$$

The element $\langle x, y\rangle$ is the unique element in $\mathcal{G}$ with $x\langle x, y\rangle=y$. By our assumption, $\mathcal{X}$ is a tight correspondence. That is, $r_{*}: \mathcal{X} / \mathcal{G} \rightarrow \mathcal{G}^{0}$ is a homeomorphism. Therefore, we may replace $\mathcal{X} / \mathcal{G}$ in Equation (3.2) with $\mathcal{G}^{0}$. Hence we obtain a map

$$
\mathcal{X} \times_{r, \mathcal{G}^{0}, r} \mathcal{X} \rightarrow \mathcal{G}, \quad(x, y) \mapsto\langle x, y\rangle .
$$

The existence of the map in Equation (3.3) for a tight correspondence is the main feature which allows to construct a colimit groupoid for a given Ore monoid action by tight correspondences.

Definition 3.5. Let $\mathcal{G}, \mathcal{H}$ be locally compact groupoids. Let $\mathcal{X}: \mathcal{G} \rightarrow \mathcal{H}$ be a correspondence. We say that an open subset $U \subset \mathcal{X}$ is a bisection if the restrictions of the source map $s_{\mathcal{X}}$ and the quotient map $\mathrm{p}: \mathcal{X} \rightarrow \mathcal{X} / \mathcal{G}$ to $U$ are both homeomorphisms.

Our definition implies that a bisection should meet every orbit of the right action exactly once. We denote the set of all bisections of a correspondence $\mathcal{X}$ by $\operatorname{Bis}(\mathcal{X})$. For a groupoid $\mathcal{G}$, a bisection in the identity correspondence $\mathcal{G}: \mathcal{G} \rightarrow \mathcal{G}$ is the same as a bisection in the groupoid $\mathcal{G}$. That is, an open set in $\mathcal{G}$ such that the restriction of the source and range maps to this set are homeomorphisms.

In the following, we fix a groupoid $\mathcal{G}$, an Ore monoid $P$ and an action $\left(\mathcal{X}_{p}, \sigma_{p, q}\right)$ of $P$ on $\mathcal{G}$ by tight correspondences. That is, $\mathcal{X}_{p}$ is a tight correspondence for all $p \in P$ and $\sigma_{p, q}: \mathcal{X}_{p q} \rightarrow \mathcal{X}_{p} \circ_{\mathcal{G}} \mathcal{X}_{q}$ is an isomorphism for all $p$ and $q \in P$. To reduce notation, we write

$$
x_{p} \cdot x_{q}:=\sigma_{p, q}^{-1}\left(\left[x_{p}, x_{q}\right]\right)
$$

for all $\left(x_{p}, x_{q}\right) \in \mathcal{X}_{p} \times_{s_{p}, \mathcal{G}^{0}, r_{q}} \mathcal{X}_{q}$. We call $x_{p} \cdot x_{q} \in \mathcal{X}_{p q}$ the concatenation of $x_{p}$ and $x_{q}$. Let $\mathcal{X}_{p} \circ \mathcal{G} \mathcal{X}_{q}^{*}$ be the quotient of the space $\mathcal{X}_{p} \times{ }_{s_{p}, \mathcal{G}^{0}, s_{q}} \mathcal{X}_{q}$ by the equivalence relation $\left(x_{p}, y_{q}\right) \sim\left(x_{p} g, y_{q} g\right)$ for all $g \in \mathcal{G}$ with $r(g)=s_{p}\left(x_{p}\right)$.

Let $k \in P$. Let $\alpha_{p, q}^{k}$ be the map

$$
\alpha_{p, q}^{k}: \mathcal{X}_{p} \times_{s_{p}, \mathcal{G}^{0}, s_{q}} \mathcal{X}_{q} \rightarrow \mathcal{X}_{p k} \circ_{\mathcal{G}} \mathcal{X}_{q k}^{*}, \quad\left(x_{p}, x_{q}\right) \mapsto\left[x_{p} \cdot x_{k}, x_{q} \cdot x_{k}\right],
$$




\section{Actions of Ore monoids}

where $x_{k} \in \mathcal{X}_{k}$ is such that $r_{k}\left(x_{k}\right)=s_{p}\left(x_{p}\right)$. To see that (3.5) does not depend on the choice of the point $x_{k}$ with $r_{k}\left(x_{k}\right)=s_{p}\left(x_{p}\right)$, let $y_{k} \in \mathcal{X}_{k}$ be another point with $r_{k}\left(y_{k}\right)=s_{p}\left(x_{p}\right)=r_{k}\left(x_{k}\right)$. Since $y_{k}\left\langle y_{k}, x_{k}\right\rangle=x_{k}$, we have

$$
\begin{aligned}
{\left[x_{p} \cdot x_{k}, x_{q} \cdot x_{k}\right] } & =\left[x_{p} \cdot\left(y_{k}\left\langle y_{k}, x_{k}\right\rangle\right), x_{q} \cdot\left(y_{k}\left\langle y_{k}, x_{k}\right\rangle\right)\right] \\
& =\left[\left(x_{p} \cdot y_{k}\right)\left\langle y_{k}, x_{k}\right\rangle,\left(x_{q} \cdot y_{k}\right)\left\langle y_{k}, x_{k}\right\rangle\right]=\left[x_{p} \cdot y_{k}, x_{q} \cdot y_{k}\right] .
\end{aligned}
$$

In particular, for $k=1$, the last formula implies that

$$
\alpha_{p, q}^{k}\left(x_{p} g, x_{q} g\right)=\alpha_{p, q}^{k}\left(x_{p}, x_{q}\right) .
$$

for all $x_{p}, x_{q}$ and all $g \in \mathcal{G}$ with $r(g)=s_{p}\left(x_{p}\right)$. Therefore, $\alpha_{p, q}^{k}$ induces a welldefined $\operatorname{map} \alpha_{p, q}^{k}: \mathcal{X}_{p} \circ \mathcal{X}_{q}^{*} \rightarrow \mathcal{X}_{p k} \circ \mathcal{X}_{q k}^{*}$.

Lemma 3.6. The map $\alpha_{p, q}^{k}: \mathcal{X}_{p} \circ \mathcal{X}_{q}^{*} \rightarrow \mathcal{X}_{p k} \circ \mathcal{X}_{q k}^{*}$ is a local homeomorphism. Moreover,

$$
\alpha_{p k, q k}^{l} \circ \alpha_{p, q}^{k}=\alpha_{p, q}^{k l}
$$

for all $p, q, k, l \in P$.

Proof. Let $x=\left[x_{p}, x_{q}\right] \in \mathcal{X}_{p} \circ_{\mathcal{G}} \mathcal{X}_{q}^{*}$. We want an open subset $U \subset \mathcal{X}_{p} \circ_{\mathcal{G}} \mathcal{X}_{q}^{*}$ such that $x \in U$ and the restriction map $\left.\alpha_{p, q}^{k}\right|_{U}$ is a homeomorphism. Let $U_{1} \subset \mathcal{X}_{p}$ and $U_{2} \subset \mathcal{X}_{q}$ be bisections with $x_{p} \in U_{1}$ and $x_{q} \in U_{2}$. We claim that $U:=$ $\mathrm{p}\left(U_{1} \times_{s_{p}, \mathcal{G}^{0}, r_{q}} U_{2}\right) \subset \mathcal{X}_{p} \circ_{\mathcal{G}} \mathcal{X}_{q}^{*}$ is the desired open set. Let $V:=r_{k}^{-1}\left(s_{p}\left(U_{1}\right) \cap\right.$ $\left.s_{q}\left(U_{2}\right)\right)$. Then $V$ is open in $\mathcal{X}_{k}$ since $s_{p}$ is a local homeomorphism and $r_{k}$ is continuous. We may write $V$ as a union of bisections $V=\bigcup_{i \in I} V_{i}$ for an index set $I$. Let $Q: \mathcal{X}_{p k} \times_{s_{p k}, \mathcal{G}^{0}, s_{q k}} \mathcal{X}_{q k} \rightarrow \mathcal{X}_{p k} \circ_{\mathcal{G}} \mathcal{X}_{q k}^{*}$ denote the quotient map. We define

$$
T_{i}:=Q\left(\sigma_{p, k}^{-1} \cdot \mathrm{p}\left(U_{1} \times_{s_{p}, \mathcal{G}^{0}, r_{k}} V_{i}\right) \times_{s_{p k}, \mathcal{G}^{0}, s_{q k}} \sigma_{q, k}^{-1} \cdot \mathrm{p}\left(U_{2} \times_{s_{p}, \mathcal{G}^{0}, r_{k}} V_{i}\right)\right)
$$

for all $i \in I$. Since $\sigma_{p, k}$ and $\sigma_{q, k}$ are homeomorphisms and the quotient maps $\mathrm{p}$ and $Q$ are open by Proposition 2.10, $T_{i}$ is open for all $i \in I$. Furthermore,

$$
\alpha_{p, q}^{k}(U)=\bigcup_{i \in I} T_{i}
$$

To see this, let $i \in I$ and let $\left[x_{p} \cdot x_{k}, y_{p} \cdot y_{k}\right] \in T_{i}$. Then $s_{k}\left(x_{k}\right)=s_{k}\left(y_{k}\right)$ and hence $x_{k}=y_{k}$ since $V_{i}$ is a bisection. This implies that $\left[x_{p} \cdot x_{k}, y_{p} \cdot y_{k}\right] \in \alpha_{p, q}^{k}(U)$. The other implication is clear. It follows that $\alpha_{p, q}^{k}(U)$ is an open set. Since every open set in $\mathcal{X}_{p} \circ_{\mathcal{G}} \mathcal{X}_{q}^{*}$ is a union of bisections of the form $\mathrm{p}\left(U_{1} \times_{s_{p}, \mathcal{G}^{0}, r_{q}} U_{2}\right)$ for $U_{1} \in \mathcal{X}_{p}$ and $U_{2} \in \mathcal{X}_{q}$, the map $\alpha_{p, q}^{k}$ is open. It remains to show that $\left.\alpha_{p, q}^{k}\right|_{U}$ is injective. Let $\left[x_{p}, x_{q}\right],\left[y_{p}, y_{q}\right] \in \mathrm{p}\left(U_{1} \times_{s_{p}, \mathcal{G}^{0}, s_{q}} U_{2}\right)$ with $\alpha_{p, q}^{k}\left(\left[x_{p}, x_{q}\right]\right)=\alpha_{p, q}^{k}\left(\left[y_{p}, y_{q}\right]\right)$. 
Then $r_{p}\left(x_{p}\right)=r_{p}\left(y_{p}\right)$ and $r_{q}\left(x_{q}\right)=r_{q}\left(y_{q}\right)$. This implies that $x_{p}=y_{p}$ and $x_{q}=y_{q}$ by our assumption that $\left.r_{p}\right|_{U_{1}}$ and $\left.r_{q}\right|_{U_{2}}$ are homeomorphisms. It follows that $\left[x_{p}, x_{q}\right]=\left[y_{p}, y_{q}\right]$, and the map $\alpha_{p, q}^{k}$ is injective on $\mathrm{p}\left(U_{1} \times_{s_{p}, \mathcal{G}^{0}, s_{q}} U_{2}\right)$. The restriction $\left.\alpha_{p, q}^{k}\right|_{U}$ of $\alpha_{p, q}^{k}$ on the open set $U$ is open, injective and continuous. That is, the map $\left.\alpha_{p, q}^{k}\right|_{U}$ is a homeomorphism. Finally, the maps $\left(\sigma_{p, q}\right)_{p, q \in P}$ are part of an action of $P$ on the groupoid $\mathcal{G}$. Hence they satisfy condition (3.1). This implies Equation (3.6).

\subsubsection{The construction of the colimit groupoid}

Next we associate a groupoid $\mathcal{H}$ to the action $\left(\mathcal{X}_{p}, \sigma_{p, q}\right)$. We construct the groupoid $\mathcal{H}$ by first constructing a "fibre" $\mathcal{H}_{g}$ for all $g \in G=G[P]$. Recall that the elements of the group completion $G=G[P]$ are equivalence classes of formal fractions $p_{1} p_{2}^{-1}$ for $p_{1}, p_{2} \in P$ with $p_{1} p_{2}^{-1} \sim\left(p_{1} r\right)\left(p_{2} r\right)^{-1}$. For $g \in G$ we set

$$
R_{g}:=\left\{\left(p_{1}, p_{2}\right) \in P \times P \mid p_{1} p_{2}^{-1}=g \text { in } G\right\} .
$$

Let $p=\left(p_{1}, p_{2}\right)$ and $q=\left(q_{1}, q_{2}\right) \in R_{g}$. Let $\mathcal{C}_{P}^{g}(p, q)$ be the set of all $h \in P$ with $p_{1} h=q_{1}$ and $p_{2} h=q_{2}$. These are the arrows of a category $\mathcal{C}_{P}^{g}$ with multiplication given by that in $P$. The category $\mathcal{C}_{P}^{g}$ is filtered by [1, Lemma 3.14]. This allows us to take the inductive limit of the spaces $\mathcal{X}_{p} \circ_{\mathcal{G}} \mathcal{X}_{q}^{*}$ with respect to the connection maps $\alpha_{p, q}^{k}$ given in Equation (3.5). We set

$$
\left(\mathcal{H}_{g}, f_{p, q}\right):=\underset{\lim _{P}^{g}}{\operatorname{li}}\left(\mathcal{X}_{p} \circ_{\mathcal{G}} \mathcal{X}_{q}^{*}, \alpha_{p, q}^{k}\right),
$$

where $f_{p, q}: \mathcal{X}_{p} \circ_{\mathcal{G}} \mathcal{X}_{q}^{*} \rightarrow \mathcal{H}_{g}$ is the universal map associated to the inductive limit space $\mathcal{H}_{g}$, for all $(p, q) \in \mathcal{C}_{P}^{g}$. Let

$$
\mathcal{H}:=\bigsqcup_{g \in G} \mathcal{H}_{g}
$$

Elements of $\mathcal{H}$ are equivalence classes of pairs $\left[x_{p}, x_{q}\right] \in \mathcal{X}_{p} \circ_{\mathcal{G}} \mathcal{X}_{q}^{*}$, where $\left[x_{p}, x_{q}\right] \sim$ $\alpha_{p, q}^{k}\left(\left[x_{p}, x_{q}\right]\right)$ for all $k \in P$. We will use this identification and, with a slight abuse of notation, we will abbreviate $f_{p, q}\left(\left[x_{p}, x_{q}\right]\right)$ by $\left[x_{p}, x_{q}\right]$ if no confusion can arise.

Our next goal is to show that the space $\mathcal{H}$ is in a canonical way a locally compact groupoid with Hausdorff unit space $\mathcal{H}^{0}$.

Let $g \in G$ and let $x=\left[x_{p}, x_{q}\right] \in \mathcal{H}_{g}$. We define the source and the range of $x$ by

$$
\begin{aligned}
r_{\mathcal{H}}(x) & :=\left[x_{p}, x_{p}\right]=\left[r_{p}\left(x_{p}\right), r_{p}\left(x_{p}\right)\right], \\
s_{\mathcal{H}}(x) & :=\left[x_{q}, x_{q}\right]=\left[r_{q}\left(x_{q}\right), r_{q}\left(x_{q}\right)\right] .
\end{aligned}
$$

That is, $\left[x_{p}, x_{p}\right]$ is the image of the point $\left[x_{p}, x_{p}\right] \in \mathcal{X}_{p} \circ_{\mathcal{G}} \mathcal{X}_{p}^{*}$ under the map $f_{p, p}$.

Before defining a multiplication map on the groupoid $\mathcal{H}$, we need the following lemma regarding the inner product map. 
Lemma 3.7. Let $p, t, k \in P$. Then

$$
x_{t}\left\langle x_{p} \cdot x_{t}, y_{p} \cdot x_{k}\right\rangle=\left\langle x_{p}, y_{p}\right\rangle x_{k}
$$

for all $\left(x_{p}, y_{p}\right) \in \mathcal{X}_{p} \times_{r_{p}, \mathcal{G}^{0}, r_{p}} \mathcal{X}_{p}$ and all $x_{t} \in \mathcal{X}_{t}, x_{k} \in \mathcal{X}_{k}$ with $s_{p}\left(x_{p}\right)=r\left(x_{t}\right)$ and $s_{p}\left(y_{p}\right)=r\left(x_{k}\right)$.

Proof. We have $x_{p}\left\langle x_{p}, y_{p}\right\rangle=y_{p}$ and $x_{p} \cdot x_{t} \cdot\left\langle x_{p} \cdot x_{t}, y_{p} \cdot x_{k}\right\rangle=y_{p} \cdot x_{k}$. This implies

$$
y_{p} \cdot\left\langle x_{p}, y_{p}\right\rangle^{-1} x_{t}\left\langle x_{p} \cdot x_{t}, y_{p} \cdot x_{k}\right\rangle=y_{p} \cdot x_{k}
$$

It follows that $\left\langle x_{p}, y_{p}\right\rangle^{-1} x_{t}\left\langle x_{p} \cdot x_{t}, y_{p} \cdot x_{k}\right\rangle=x_{k}$ since the right action of $\mathcal{G}$ on $\mathcal{X}_{p}$ is free. Hence $x_{t}\left\langle x_{p} \cdot x_{t}, y_{p} \cdot x_{k}\right\rangle=\left\langle x_{p}, y_{p}\right\rangle x_{k}$.

Now let $x=\left[x_{p_{1}}, x_{q_{1}}\right]$ and $y=\left[y_{p_{2}}, y_{q_{2}}\right] \in \mathcal{H}$ with $r_{p_{2}}\left(y_{p_{2}}\right)=r_{q_{1}}\left(x_{q_{1}}\right)$. Roughly speaking, we want to define the product $x \cdot y$ as for pair groupoids. Think of $x$ as the image in $\mathcal{H}$ of an arrow starting with $x_{q_{1}}$ and ending with $x_{p_{1}}$. Similarly, $y$ is the image of the arrow starting with $y_{q_{2}}$ and ending with $x_{p_{2}}$. If $p_{2}=q_{1}$ and $y_{p_{2}}=x_{q_{1}}$, then we may define the product $x \cdot y$ as $\left[x_{p_{1}}, y_{q_{2}}\right]$. In general, if the range of $y$ is the source of $x$, we may choose different representatives of $x$ and $y$ with this property. To be more precise, since $P$ is an Ore monoid, there are $a, b \in P$ such that $p_{2} b=q_{1} a$. Let $x_{a} \in \mathcal{X}_{a}$ and $y_{b} \in \mathcal{X}_{b}$ with $r_{a}\left(x_{a}\right)=s_{p_{1}}\left(x_{p_{1}}\right)$ and $r_{b}\left(y_{b}\right)=s_{p_{2}}\left(y_{p_{2}}\right)$. Then $\left[x_{p_{1}}, x_{q_{1}}\right]=\left[x_{p_{1}} \cdot x_{a}, x_{q_{1}} \cdot x_{a}\right]$ and $\left[y_{p_{2}}, y_{q_{2}}\right]=\left[y_{p_{2}} \cdot y_{b}, y_{q_{2}} \cdot y_{b}\right]$. We define

$$
\left[x_{p_{1}}, x_{q_{1}}\right] \cdot\left[y_{p_{2}}, y_{q_{2}}\right]:=\left[x_{p_{1}} \cdot x_{a} \cdot\left\langle x_{q_{1}} \cdot x_{a}, y_{p_{2}} \cdot y_{b}\right\rangle, y_{q_{2}} \cdot y_{b}\right]
$$

The product formula (3.11) does not depend on the choice of the representatives $\left[x_{p_{1}}, x_{q_{1}}\right] \in \mathcal{X}_{p_{1}} \circ_{\mathcal{G}} \mathcal{X}_{q_{1}}$ and $\left[y_{p_{2}}, y_{q_{2}}\right] \in \mathcal{X}_{p_{2}} \circ_{\mathcal{G}} \mathcal{X}_{q_{2}}$ in $\mathcal{H}$. To show this, let $k_{1}, k_{2} \in P$. Let also $x_{k_{1}} \in \mathcal{X}_{k_{1}}$ and $x_{k_{2}} \in \mathcal{X}_{k_{2}}$ such that $r_{k_{1}}\left(x_{k_{1}}\right)=s_{p_{1}}\left(x_{p_{1}}\right)$ and $r_{k_{2}}\left(x_{k_{2}}\right)=s_{p_{2}}\left(x_{p_{2}}\right)$. We have

$$
\left(\left[x_{p_{1}} \cdot x_{k_{1}}, x_{q_{1}} \cdot x_{k_{1}}\right],\left[y_{p_{2}} \cdot x_{k_{2}}, y_{q_{2}} \cdot x_{k_{2}}\right]\right)=\left(\left[x_{p_{1}}, x_{q_{1}}\right],\left[y_{p_{2}}, y_{q_{2}}\right]\right) \in \mathcal{H}^{2} .
$$

Next let $c_{1}, c_{2}, d_{1}, d_{2}$ be such that $k_{1} c_{1}=a d_{1}$ and $k_{2} c_{2}=b d_{2}$. We can also find $t_{1}, t_{2}$ such that $d_{1} t_{1}=d_{2} t_{2}$. Then $q_{1} k_{1} c_{1} t_{1}=q_{1} a d_{1} t_{1}=p_{2} b d_{2} t_{2}=p_{2} k_{2} c_{2} t_{2}$. We may replace $c_{1} t_{1}$ by $c_{1}, c_{2} t_{2}$ by $c_{2}$ and $d_{2} t_{2}=d_{1} t_{1}$ by $t$. We get $k_{1} c_{1}=a t, k_{2} c_{2}=b t$ and $q_{1} k_{1} c_{1}=q_{1} a t=p_{2} b t=q_{2} k_{2} c_{2}$. Let also $x_{t} \in \mathcal{X}_{t}$ with $r\left(x_{p}\right)=s\left(x_{a}\right)=s\left(x_{b}\right)$. Then $x_{a} \cdot x_{t} \in \mathcal{X}_{a t}=\mathcal{X}_{k_{1} c_{1}}$ and $x_{b} \cdot x_{t} \in \mathcal{X}_{b t}=\mathcal{X}_{k_{2} c_{2}}$. It follows that $x_{a} \cdot x_{t}=x_{k_{1}} \cdot x_{c_{1}}$ and $x_{b} \cdot x_{t}=x_{k_{2}} \cdot x_{c_{2}}$, where $\left[x_{k_{1}}, x_{c_{1}}\right]=\sigma_{k_{1}, c_{1}}\left(x_{a} \cdot x_{t}\right)$ and $\left[x_{k_{2}}, x_{c_{2}}\right]=\sigma_{k_{2}, c_{2}}\left(x_{b} \cdot x_{t}\right)$. 
Lemma 3.7 implies

$$
\begin{aligned}
& {\left[x_{p_{1}} \cdot x_{k_{1}} \cdot x_{c_{1}}, x_{q_{1}} \cdot x_{k_{1}} \cdot x_{c_{1}}\right] \cdot\left[y_{p_{2}} \cdot x_{k_{2}} \cdot x_{c_{2}}, y_{q_{2}} \cdot x_{k_{2}} \cdot x_{c_{2}}\right]} \\
& =\left[x_{p_{1}} \cdot x_{k_{1}} \cdot x_{c_{1}} \cdot\left\langle x_{q_{1}} \cdot x_{k_{1}} \cdot x_{c_{1}}, y_{p_{2}} \cdot x_{k_{2}} \cdot x_{c_{2}}\right\rangle, y_{q_{2}} \cdot x_{k_{2}} \cdot x_{c_{2}}\right] \\
& =\left[x_{p_{1}} \cdot x_{a} \cdot x_{t} \cdot\left\langle x_{q_{1}} \cdot x_{a} \cdot x_{t}, y_{p_{2}} \cdot y_{b} \cdot x_{t}\right\rangle, y_{q_{2}} \cdot y_{b} \cdot x_{t}\right] \\
& =\left[x_{p_{1}} \cdot x_{a} \cdot\left\langle x_{q_{1}} \cdot x_{a}, y_{p_{2}} \cdot y_{b}\right\rangle x_{t}, y_{q_{2}} \cdot y_{b} \cdot x_{t}\right] \\
& =\left[x_{p_{1}} \cdot x_{a} \cdot\left\langle x_{q_{1}} \cdot x_{a}, y_{p_{2}} \cdot y_{b}\right\rangle, y_{q_{2}} \cdot y_{b}\right] \\
& =\left[x_{p_{1}}, x_{q_{1}}\right] \cdot\left[y_{p_{2}}, y_{q_{2}}\right] .
\end{aligned}
$$

Therefore, the product formula gives a well-defined map $\mathcal{H}^{2} \rightarrow \mathcal{H}$.

Finally, we define the inversion in $\mathcal{H}$ by

$$
\left[x_{p}, x_{q}\right]^{-1}:=\left[x_{q}, x_{p}\right]
$$

for all $\left[x_{p}, x_{q}\right] \in \mathcal{H}$.

Lemma 3.8. The map $\beta: \mathcal{H}^{0} \rightarrow \mathcal{G}^{0},\left[x_{p}, x_{p}\right] \mapsto r_{p}\left(x_{p}\right)$, is a homeomorphism.

Proof. We have $\left[x_{p}, x_{p}\right]=\left[r_{p}\left(x_{p}\right) \cdot x_{p}, r_{p}\left(x_{p}\right) \cdot x_{p}\right]=\left[r_{p}\left(x_{p}\right), r_{p}\left(x_{p}\right)\right]$ for all $\left[x_{p}, x_{p}\right] \in$ $\mathcal{H}$. This implies that the the map $\beta$ is a bijection. It is open since the range map $r_{p}$ is open in the groupoid $\mathcal{G}$ and $\mathcal{H}^{0} \subset \mathcal{H}_{1}$ has the inductive limit topology. To show that it is continuous, it suffices to see that the set

$$
f_{p, p}^{-1}\left(\beta^{-1}(U)\right)=\left\{\mathbf{p}_{p, p}\left(x_{p}, x_{p}\right) \mid\left(x_{p}, x_{p}\right) \in r_{p}^{-1}(U) \times_{s_{p}, \mathcal{G}^{0}, s_{p}} r_{p}^{-1}(U)\right\}
$$

is open if $U \subset \mathcal{G}^{0}$ is open. This follows since $r_{p}$ is continuous for all $p \in P$.

Lemma 3.9. For all $g \in G$ and all $(p, q) \in \mathcal{C}_{P}^{g}$, the map $f_{p, q}: \mathcal{X}_{p} \circ_{\mathcal{G}} \mathcal{X}_{q}^{*} \rightarrow \mathcal{H}_{g}$ is a local homeomorphism.

Proof. The map $f_{p, q}$ is open and continuous since the maps $\alpha_{p, q}^{k}$ are local homeomorphisms for all $p, q, k \in P$ by Lemma 3.6 and the space $\mathcal{H}_{g}$ carries the inductive limit topology. The proof that every point $\left[x_{p}, x_{q}\right]$ in $\mathcal{X}_{p} \circ_{\mathcal{G}} \mathcal{X}_{q}^{*}$ has a neighborhood $U$ such that the restriction of $f_{p, q}$ to $U$ is injective is as in the proof of Lemma 3.6.

For simplicity, we write $\iota_{p}:=f_{p, 1}: \mathcal{X}_{p} \rightarrow \mathcal{H}$. That is,

$$
\iota_{p}\left(x_{p}\right)=f_{p, 1}\left(\left[x_{p}, s_{p}\left(x_{p}\right)\right]\right) .
$$

Proposition 3.10. With the groupoid structure defined above, $\mathcal{H}$ is an étale locally compact groupoid with Hausdorff unit space. 


\section{Actions of Ore monoids}

Proof. Let $U$ be an open set in $\mathcal{H}$. Without loss of generality, we may assume that $U=f_{p, q}\left(U_{1} \circ_{\mathcal{G}} U_{2}^{*}\right)$ for open sets $U_{1} \subset \mathcal{X}_{p}$ and $U_{2} \subset \mathcal{X}_{q}$. The inverse image of the set $U$ with respect to the multiplication map defined in Equation (3.11) is

$$
\bigcup_{k \in P} \mathrm{p}\left(U_{1} \times_{s_{p}, \mathcal{G}^{0}, s_{k}} \mathcal{X}_{k}\right) \times_{r_{k}, \mathcal{G}^{0}, r_{k}} \mathrm{p}\left(\mathcal{X}_{k} \times_{s_{p}, \mathcal{G}^{0}, s_{q}} U_{2}\right) ;
$$

this is open since the quotient maps $\mathrm{p}$ are open by Proposition 2.10. This implies that Equation (3.11) defines a continuous map. The inverse image of $U$ with respect to the inversion defined in Equation (3.12) is

$$
f_{q, p}\left(U_{2} \circ_{\mathcal{G}} U_{1}^{*}\right),
$$

which is open in $\mathcal{H}$. Hence the inversion $\mathcal{H} \rightarrow \mathcal{H}$ is also continuous.

Now we show that $\mathcal{H}$ is étale. For all $p \in P$, the map $r_{p}$ is a local homeomorphism by Lemma 2.20. Let $\left[x_{p}, x_{q}\right] \in \mathcal{H}$ for $p$ and $q \in P$. Then there are bisections $U \subset \mathcal{X}_{p}$ and $V \in \mathcal{X}_{q}$ with $x_{p} \in U$ and $x_{q} \in V$. We set

$$
M:=f_{p, q}\left(U \circ V^{*}\right)=\left\{\left[y_{p}, y_{q}\right] \in \mathcal{H} \mid y_{p} \in U, y_{q} \in V\right\} .
$$

Then $M$ is an open set; this follows since $f_{p, q}$ is open by Lemma 3.9. Moreover, for all $\left[y_{p}, y_{q}\right] \in M$ we have

$$
r_{\mathcal{H}}\left(\left[y_{p}, y_{q}\right]\right)=\beta^{-1}\left(r_{p}\left(y_{p}\right)\right), \quad s_{\mathcal{H}}\left(\left[y_{p}, y_{q}\right]\right)=\beta^{-1}\left(r_{q}\left(y_{q}\right)\right) .
$$

This implies that the maps $r_{\mathcal{H}}$ and $s_{\mathcal{H}}$ are local homeomorphisms by Lemma 2.20 and Lemma 3.8. In addition, Lemma 3.8 implies that $\mathcal{H}^{0}$ is Hausdorff since it is homeomorphic to $\mathcal{G}^{0}$, which is Hausdorff by assumption.

Before proving that the groupoid $\mathcal{H}$ is the colimit of the diagram $\left(\mathcal{X}_{p}, \sigma_{p, q}\right)$ we prove the following results regarding the maps $f_{p, q}$.

Lemma 3.11. Let $g \in G$ and let $(p, q) \in \mathcal{C}_{P}^{g}$. The map $f_{p, q}: \mathcal{X}_{p} \circ_{\mathcal{G}} \mathcal{X}_{q}^{*} \rightarrow \mathcal{H}_{g}$ is injective if the left action of $\mathcal{G}$ on $\mathcal{X}_{k}$ is free for all $k \in P$.

Proof. Let $\left[x_{p}, x_{q}\right],\left[y_{p}, y_{q}\right] \in \mathcal{X}_{p} \circ \mathcal{G} \mathcal{X}_{q}^{*}$ be such that $f_{p, q}\left(\left[x_{p}, x_{q}\right]\right)=f_{p, q}\left(\left[y_{p}, y_{q}\right]\right)$. Then there is an element $k \in P$ and there are elements $x_{k}$ and $y_{k} \in \mathcal{X}_{k}$ with $r_{k}\left(x_{k}\right)=s_{p}\left(x_{p}\right)$ and $r_{k}\left(y_{k}\right)=s_{p}\left(y_{p}\right)$ and such that

$$
\left[x_{p} \cdot x_{k}, x_{q} \cdot x_{k}\right]=\left[y_{p} \cdot y_{k}, y_{q} \cdot y_{k}\right] .
$$

Hence there is an element $g \in \mathcal{G}$ such that

$$
x_{p} \cdot x_{k}=y_{p} \cdot y_{k} g, \quad x_{q} \cdot x_{k}=y_{q} \cdot y_{k} g .
$$

This implies that there are $h_{1}, h_{2} \in \mathcal{G}$ such that $x_{p}=y_{p} h_{1}, x_{k}=h_{1}^{-1} y_{k} g, x_{q}=$ $y_{q} h_{2}$ and $x_{k}=h_{2}^{-1} y_{k} g$. It follows that $h_{1}^{-1} y_{k} g=h_{2}^{-1} y_{k} g$ and therefore $h_{1}=$ $h_{2}$ by our assumption that the left action of $\mathcal{G}$ on $\mathcal{X}_{k}$ is free for all $k \in P$. Consequently, $\left[x_{p}, x_{q}\right]=\left[y_{p} h_{1}, y_{q} h_{1}\right]=\left[y_{p}, y_{q}\right]$ and the map $f_{p, q}$ is injective. 
Lemma 3.12. The map $\iota_{1}: \mathcal{G} \rightarrow \mathcal{H}$ is a groupoid homomorphism. It is injective if and only if the left action of $\mathcal{G}$ on $\mathcal{X}_{k}$ is free for all $k \in P$.

Proof. Let $g$ and $h \in \mathcal{G}$. We need to show that $\iota_{1}(g)^{-1}=\iota_{1}\left(g^{-1}\right)$ and $\iota_{1}(g h)=$ $\iota_{1}(g) \iota_{1}(h)$. The first claim follows since

$$
\iota_{1}(g)^{-1}=[g, s(g)]^{-1}=[s(g), g]=\left[s(g) g^{-1}, g g^{-1}\right]=\left[g^{-1}, r(g)\right]=\iota_{1}\left(g^{-1}\right)
$$

for all $g \in \mathcal{G}$. And the second claim follows since

$$
\iota_{1}(g) \iota_{1}(h)=[g, s(g)] \cdot[h, s(h)]=[g\langle s(g), h\rangle, s(h)]=[g h, s(h)]=\iota_{1}(g h)
$$

for all $g$ and $h \in \mathcal{G}$ with $r(h)=s(g)$. If the left action of $\mathcal{G}$ on $\mathcal{X}_{k}$ is free for all $k \in P$ then the map $\iota_{1}=f_{p, 1}$ is injective by Lemma 3.11. We assume now that the map $\iota_{1}$ is injective. Let $k \in P$ and let $\left(g, x_{k}\right) \in \mathcal{G} \times_{s, \mathcal{G}^{0}, r} \mathcal{X}_{k}$ with $g \cdot x_{k}=x_{k}$. We have

$$
\iota_{1}(g)=[g, s(g)]=\left[g x_{k}, x_{k}\right]=\left[x_{k}, x_{k}\right]=[s(g), s(g)]=\iota_{1}(s(g)) .
$$

Hence $g=s(g)$ by the injectivity of $\iota_{1}$. This implies that the left action of $\mathcal{G}$ on $\mathcal{X}_{k}$ is free for all $k \in P$.

We omit the proof of the following lemma, which uses only properties of the inner product map (see Proposition 2.26).

Lemma 3.13. The maps $\iota_{p}=f_{p, 1}$ for $p \in P$ satisfy the following properties:

(1) $\iota_{p}\left(x_{p}\right) \iota_{1}(g)=\iota_{p}\left(x_{p} g\right)$ for all $g \in \mathcal{G}$ and $x_{p} \in \mathcal{X}_{p}$ with $r(g)=s_{p}\left(x_{p}\right)$.

(2) $\iota_{1}(g) \iota_{p}\left(x_{p}\right)=\iota_{p}\left(g x_{p}\right)$ for all $g \in \mathcal{G}$ and $x_{p} \in \mathcal{X}_{p}$ with $r_{p}\left(x_{p}\right)=s(g)$.

(3) $\iota_{p}\left(x_{p}\right) \iota_{q}\left(x_{q}\right)^{*}=f_{p, q}\left(\left[x_{p}, x_{q}\right]\right)$ for all $\left[x_{p}, x_{q}\right] \in \mathcal{X}_{p} \circ_{\mathcal{G}} \mathcal{X}_{q}^{*}$.

(4) $\iota_{p}\left(x_{p}\right)^{*} \iota_{p}\left(y_{p}\right)=\iota_{1}\left(\left\langle x_{p}, y_{p}\right\rangle\right)$ for all $x_{p}$ and $y_{p} \in \mathcal{X}_{p}$ with $r_{p}\left(x_{p}\right)=r_{p}\left(y_{p}\right)$.

(5) $\iota_{p}\left(x_{p}\right) \iota_{q}\left(x_{q}\right)=\iota_{p q}\left(x_{p} \cdot x_{q}\right)$ for all $x_{p} \in \mathcal{X}_{p}$ and $x_{q} \in \mathcal{X}_{q}$ with $s_{p}\left(x_{p}\right)=r_{q}\left(x_{q}\right)$.

Remark 3.14. We have $\mathcal{H}_{1}=\left\{\left[x_{p}, y_{p}\right] \in \mathcal{H} \mid x_{p}, y_{p} \in \mathcal{X}_{p}\right\}$. This is a clopen subgroupoid of $\mathcal{H}$. To see this, let $p$ and $q \in P$ and let $\left[x_{p}, y_{p}\right] \in \mathcal{H}_{1}$ and $\left[x_{q}, y_{q}\right] \in$ $\mathcal{H}_{1}$. Let $k_{1}$ and $k_{2} \in P$ be such that $p k_{1}=q k_{2}$. Let also $x_{k_{1}}$ and $x_{k_{2}}$ be such that $r_{k_{2}}\left(x_{k_{2}}\right)=s_{q}\left(x_{q}\right)$ and $r_{k_{1}}\left(x_{k_{1}}\right)=s_{p}\left(x_{p}\right)$. Then we have

$$
\left[x_{p}, y_{p}\right] \cdot\left[x_{q}, y_{q}\right]=\left[x_{p} \cdot x_{k_{1}} \cdot\left\langle y_{p} \cdot x_{k_{1}}, x_{q} \cdot x_{k_{2}}\right\rangle, y_{q} \cdot x_{k_{2}}\right],
$$

It follows that $\left[x_{p}, y_{p}\right] \cdot\left[x_{q}, y_{q}\right] \in \mathcal{H}_{1}$. Furthermore, for all $\left[x_{p}, y_{p}\right] \in \mathcal{H}_{1}$ we have $\left[x_{p}, y_{p}\right]^{-1}=\left[y_{p}, x_{p}\right] \in \mathcal{H}_{1}$. So $\mathcal{H}_{1}$ is closed under taking inverses and multiplication. Thus $\mathcal{H}_{1}$ is a subgroupoid in $\mathcal{H}$. It is also clopen in $\mathcal{H}$. 
Theorem 3.15. Let $\mathcal{G}$ be a groupoid and let $\left(\mathcal{X}_{p}, \sigma_{p, q}\right)$ be an action of an Ore monoid $P$ by tight correspondences. Let $\mathcal{H}$ be the groupoid constructed above. Then $\mathcal{H}$ is the colimit of the diagram $\left(\mathcal{X}_{p}, \sigma_{p, q}\right)$ in $\mathfrak{G r}, \mathfrak{G r}_{\text {prop }}$ and $\mathfrak{G r}_{\text {tight }}$.

Proof. Let $\mathcal{D}$ be a groupoid and let $\left(\mathcal{Y}, \varphi_{p}\right)$ be a transformation from $\left(\mathcal{X}_{p}, \sigma_{p, q}\right)$ to const $_{D}$. That is, $\mathcal{Y}: \mathcal{G} \rightarrow \mathcal{D}$ is a proper correspondence and $\varphi_{p}: \mathcal{X}_{p} \circ_{\mathcal{G}} \mathcal{Y} \rightarrow \mathcal{Y}$ is an isomorphism of groupoid correspondences for all $p \in P$. We want to extend $\mathcal{Y}$ to a correspondence $\mathcal{Y}: \mathcal{H} \rightarrow \mathcal{D}$. The anchor map $r: \mathcal{Y} \rightarrow \mathcal{H}^{0}$ is obtained using the identification $\mathcal{H}^{0} \cong \mathcal{G}^{0}$, see Lemma 3.8. We extend the action of $\mathcal{G}$ on $\mathcal{Y}$ to an action of the groupoid $\mathcal{H}$ as follows. For all $x_{p} \in \mathcal{X}_{p}$, let $S_{x_{p}}: \mathcal{Y} \rightarrow \mathcal{Y}$ be the partial map

$$
S_{x_{p}}(y):=\varphi_{p}\left(\left[x_{p}, y\right]\right)
$$

for all $y \in \mathcal{Y}$ with $r(y)=s_{p}\left(x_{p}\right)$. Let $y_{1}, y_{2} \in \mathcal{Y}$ with $r\left(y_{1}\right)=r\left(y_{2}\right)=s_{p}\left(x_{p}\right)$ and $S_{x_{p}}\left(y_{1}\right)=S_{x_{p}}\left(y_{1}\right)$. Then $\varphi_{p}\left(x_{p}, y_{1}\right)=\varphi_{p}\left(x_{p}, y_{1}\right)$ and hence $\left[x_{p}, y_{1}\right]=\left[x_{p}, y_{2}\right] \in$ $\mathcal{X} \circ_{\mathcal{G}} \mathcal{Y}$. It follows that there is $g \in \mathcal{G}$ such that $x_{p} g^{-1}=x_{p}$ and $g y_{1}=y_{2}$. But then $g=s\left(x_{p}\right)$ since the right action of $\mathcal{G}$ on $\mathcal{X}$ is free. Hence $y_{1}=y_{2}$, and $S_{x_{p}}$ is injective. Let $S_{x_{p}}^{-1}$ be the inverse of $S_{x_{p}}$. The domain of $S_{x_{p}}^{-1}$ is the range of $S_{x_{p}}$ and it consists of all elements $y \in \mathcal{Y}$ with $r(y)=r_{p}\left(x_{p}\right)$. To see this, let $y \in \mathcal{Y}$ with $r(y)=r_{p}\left(x_{p}\right)$ be such an element and let $\left(y_{p}, y_{1}\right) \in \mathcal{X}_{p} \times_{s_{p}, \mathcal{G}^{0}, r_{\mathcal{Y}}} \mathcal{Y}$ be such that $\varphi_{p}\left(\left[y_{p}, y_{1}\right]\right)=y$. This exists because $\varphi_{p}$ is an isomorphism. We have $r_{p}\left(y_{p}\right)=r(y)$. But $r(y)=r_{p}\left(x_{p}\right)$ by assumption. Hence $r_{p}\left(x_{p}\right)=r_{p}\left(y_{p}\right)$ which implies that there is an element $g \in \mathcal{G}$ with $y_{p} g=x_{p}$ because $\mathcal{X}_{p}$ is a tight correspondence. It follows that

$$
\varphi_{q}\left(\left[y_{p}, y_{1}\right]\right)=\varphi_{q}\left(\left[y_{p} g, g^{-1} y_{1}\right]\right)=\varphi_{q}\left(\left[x_{p}, g^{-1} y_{1}\right]\right)=S_{x_{p}}\left(g^{-1} y_{1}\right)=y .
$$

The last equation also says that $S_{x_{p} g}(y)=S_{x_{p}}(g y)$ for all $g \in \mathcal{G}$ with $r(g)=s_{p}\left(x_{p}\right)$. Equivalently, $S_{x_{p} g}^{-1}(y)=g^{-1} S_{x_{p}}^{-1}(y)$ for all $g \in \mathcal{G}$ such that $r(g)=s_{p}\left(x_{p}\right)$. More generally, (2.29) implies

$$
S_{x_{p} \cdot x_{k}}(y)=\varphi_{p k}\left(x_{p} \cdot x_{k}, y\right)=\varphi_{p}\left(x_{p}, \varphi_{k}\left(x_{k}, y\right)\right)=S_{x_{p}} S_{x_{k}}(y)
$$

for all $k \in P, x_{k} \in \mathcal{X}_{k}$ and all $y \in \mathcal{Y}$ with $r_{k}\left(x_{k}\right)=s_{p}\left(x_{p}\right)$ and $r(y)=s_{p}\left(x_{p}\right)$. This implies that

$$
S_{x_{p} \cdot x_{k}}=S_{x_{p}} S_{x_{k}}
$$

for all $x_{p} \in \mathcal{X}_{p}$ and all $x_{k} \in \mathcal{X}_{k}$ with $r_{k}\left(x_{k}\right)=s_{p}\left(x_{p}\right)$.

We define a left action of $\mathcal{H}$ on $\mathcal{Y}$ by

$$
\left[x_{p}, x_{q}\right] \cdot y:=S_{x_{p}} S_{x_{q}}^{-1}(y)
$$

for all $p, q \in P$ and all $\left(\left[x_{p}, x_{q}\right], y\right) \in\left(\mathcal{X}_{p} \circ_{\mathcal{G}} \mathcal{X}_{q}^{*}\right) \times_{s_{\mathcal{H}}, \mathcal{H}^{0}, r_{\mathcal{Y}}} \mathcal{Y}$. Equation (3.15) insures that (3.16) is compatible with the inductive limit structure on $\mathcal{H}_{g}$ for all $g \in G$ and 
hence gives a well-defined action of $\mathcal{H}$ on $\mathcal{Y}$. Clearly, this action commutes with the right action of $\mathcal{D}$ on $\mathcal{X}$. Therefore, $\mathcal{Y}: \mathcal{H} \rightarrow \mathcal{D}$ is a correspondence in $\mathfrak{G r}$. If the correspondence $\mathcal{Y}: \mathcal{G} \rightarrow \mathcal{D}$ is proper (tight) then the map $r_{\mathcal{Y}_{*}}: \mathcal{Y} / \mathcal{D} \rightarrow \mathcal{H}^{0}=\mathcal{G}^{0}$ is proper (a homeomorphism); that is, the correspondence $\mathcal{Y}: \mathcal{H} \rightarrow \mathcal{D}$ is proper (tight).

Conversely, let $\mathcal{Y}: \mathcal{H} \rightarrow \mathcal{D}$ be a correspondence. We may view $\mathcal{Y}: \mathcal{H} \rightarrow \mathcal{D}$ as a correspondence $\mathcal{Y}: \mathcal{G} \rightarrow \mathcal{D}$ using the map $\iota_{1}: \mathcal{G} \rightarrow \mathcal{H}$. Recall that $\iota_{p}: \mathcal{X}_{p} \rightarrow \mathcal{H}$ is the local homeomorphism given by

$$
\iota_{p}\left(x_{p}\right)=f_{p, 1}\left(\left[x_{p}, s_{p}\left(x_{p}\right)\right]\right),
$$

see Equation (3.13). The anchor map $r^{\mathcal{G}}: \mathcal{Y} \rightarrow \mathcal{G}^{0}$ is given by

$$
r^{\mathcal{G}}(y):=\beta(r(y))
$$

where $\beta: \mathcal{H}^{0} \rightarrow \mathcal{G}^{0}$ is the homeomorphism defined in Lemma 3.8. The left action of $\mathcal{G}$ on $\mathcal{Y}$ is given by

$$
g \cdot y:=\iota_{1}(g) \cdot y=[g, s(g)] \cdot y
$$

for all $g \in \mathcal{G}, y \in \mathcal{Y}$ with $r_{\mathcal{Y}}=s(g)$. The resulting action commutes with the right action of $\mathcal{D}$ on $\mathcal{Y}$. For all $p \in P$, we define

$$
\varphi_{p}\left(x_{p}, y\right):=\iota_{p}\left(x_{p}\right) \cdot y=\left[x_{p}, \operatorname{id}_{s_{p}\left(x_{p}\right)}\right] \cdot y
$$

for all $\left(x_{p}, y\right) \in \mathcal{X}_{p} \times_{s_{p}, \mathcal{G}^{0}, r_{\mathcal{Y}}} \mathcal{Y}$. We have

$$
\begin{aligned}
\varphi_{p}\left(x_{p} g, g^{-1} \cdot y\right) & =\iota_{p}\left(x_{p} g\right) \cdot\left(\iota_{1}\left(g^{-1}\right) \cdot y\right) \\
& =\iota_{p}\left(x_{p}\right) \iota_{1}(g) \iota_{1}\left(g^{-1}\right) \cdot y=\iota_{p}\left(x_{p}\right) \cdot y=\varphi_{p}\left(x_{p}, y\right)
\end{aligned}
$$

for all $\left(x_{p}, y\right) \in \mathcal{X}_{p} \times_{s_{p}, \mathcal{G}^{0}, r_{\mathcal{Y}}} \mathcal{Y}$ and all $g \in \mathcal{G}$ with $r(g)=s_{p}\left(x_{p}\right)$ by Lemma 3.13. Thus $\varphi_{p}$ induces a well defined map $\varphi_{p}: \mathcal{X}_{p} \circ_{\mathcal{G}} \mathcal{Y} \rightarrow \mathcal{Y}$. Next we show that $\varphi_{p}$ is an isomorphism. Let $\left(x_{p}, y_{1}\right),\left(y_{p}, y_{2}\right) \in \mathcal{X}_{p} \times_{s_{p}, \mathcal{G}^{0}, r_{\mathcal{Y}}} \mathcal{Y}$ with $\varphi_{p}\left(x_{p}, y_{1}\right)=\varphi_{p}\left(y_{p}, y_{2}\right)$. Then $r_{p}\left(x_{p}\right)=r_{p}\left(y_{p}\right)$. Let $g:=\left\langle x_{p}, y_{p}\right\rangle$. We have $x_{p} g=y_{p}$. Moreover, $\iota_{p}\left(x_{p}\right) \cdot y_{1}=$ $\iota_{p}\left(y_{p}\right) \cdot y_{2}$ implies

$$
\begin{aligned}
y_{2} & =\iota_{p}\left(y_{p}\right)^{-1} \iota_{p}\left(x_{p}\right) \cdot y_{1}=\iota_{p}\left(x_{p} g\right)^{-1} \iota_{p}\left(x_{p}\right) \cdot y_{1} \\
& =\iota_{1}\left(g^{-1}\right) \iota_{p}\left(x_{p}\right)^{-1} \iota_{p}\left(x_{p}\right) \cdot y_{1}=g^{-1} \cdot y_{1} .
\end{aligned}
$$

Thus $\left[y_{p}, y_{2}\right]=\left[x_{p} g, g^{-1} \cdot y_{1}\right]=\left[x_{p}, y_{1}\right]$. Therefore, $\varphi_{p}: \mathcal{X}_{p} \circ_{\mathcal{G}} \mathcal{Y} \rightarrow \mathcal{Y}$ is injective. Now let $y \in \mathcal{Y}$. Since $r_{p}$ is surjective, there is a point $x_{p} \in \mathcal{X}_{p}$ such that $r_{\mathcal{Y}}(y)=r_{p}\left(x_{p}\right)$. We set $y^{\prime}:=\iota_{p}\left(x_{p}\right)^{-1} \cdot y=\left[\operatorname{id}_{s_{p}\left(x_{p}\right)}, x_{p}\right] \cdot y$. Then

$$
\varphi_{p}\left(x_{p}, y^{\prime}\right)=\iota_{p}\left(x_{p}\right) \cdot y^{\prime}=\iota_{p}\left(x_{p}\right) \iota_{p}\left(x_{p}\right)^{-1} \cdot y=y
$$




\section{Actions of Ore monoids}

So $\varphi_{p}$ is a bijection. Furthermore, we have

$$
\varphi_{p}\left(g x_{p}, y\right)=\iota_{p}\left(g x_{p}\right) \cdot y=\iota_{1}(g) \iota_{p}\left(x_{p}\right) \cdot y=g \cdot \varphi_{p}\left(x_{p}, y\right)
$$

for all $\left(x_{p}, y\right) \in \mathcal{X}_{p} \times_{s_{p}, \mathcal{G}^{0}, r_{\mathcal{Y}}} \mathcal{Y}$ and all $g \in \mathcal{G}$ with $s(g)=r_{p}\left(x_{p}\right)$. This implies that $\varphi_{p}$ is $\mathcal{G}$-equivariant. It is also $\mathcal{D}$-equivariant since $\mathcal{Y}: \mathcal{H} \rightarrow \mathcal{D}$ is a correspondence and the right and left actions on $\mathcal{Y}$ commute. The map $\varphi_{p}$ is the map induced by multo $\left(\iota_{p} \times\right.$ id $): \mathcal{X}_{p} \times_{s_{p}, \mathcal{G}^{0}, r_{\mathcal{Y}}} \mathcal{Y} \rightarrow \mathcal{H} \times_{s, \mathcal{G}^{0}, r_{\mathcal{Y}}} \mathcal{Y} \rightarrow \mathcal{Y}$ on the quotient space $\mathcal{X}_{p} \circ_{\mathcal{G}} \mathcal{Y}$. The map $\iota_{p} \times$ id: $\mathcal{X}_{p} \times_{s_{p}, \mathcal{G}^{0}, r_{\mathcal{Y}}} \mathcal{Y} \rightarrow \mathcal{H} \times_{s, \mathcal{H}^{0}, r_{\mathcal{Y}}} \mathcal{Y}$ is a local homeomorphism (see Lemma 3.9). The multiplication map mult : $\mathcal{H} \times_{s, \mathcal{H}^{0}, r \mathcal{Y}} \mathcal{Y} \rightarrow \mathcal{Y}$ is a local homeomorphism as well. Hence the product map mult $\circ\left(\iota_{p} \times\right.$ id) is a local homeomorphism, and so is its induced map on the space $\mathcal{X}_{p} \circ_{\mathcal{G}} \mathcal{Y}$. It follows that $\varphi_{p}$ is an isomorphism of correspondences for all $p \in P$.

Next let $p, q \in P$ and let $x_{p} \in \mathcal{X}_{p}, x_{q} \in \mathcal{X}_{q}$ and $y \in \mathcal{Y}$ with $r_{\mathcal{Y}}(y)=s_{q}\left(x_{q}\right)$ and $r_{q}\left(x_{q}\right)=s_{p}\left(x_{p}\right)$. Then

$$
\varphi_{p q}\left(x_{p} \cdot x_{q}, y\right)=\iota_{p q}\left(x_{p} \cdot x_{q}\right) \cdot y=\iota_{p}\left(x_{p}\right) \iota_{q}\left(x_{q}\right) \cdot y=\varphi_{p}\left(x_{p}, \varphi_{q}\left(x_{q}, y\right)\right) .
$$

Hence the coherence condition (2.29) holds. This implies that $\left(\mathcal{Y}, \varphi_{p}\right)$ is a transformation from $\left(\mathcal{X}_{p}, \sigma_{p, q}\right)$ to the constant diagram over $\mathcal{D}$.

This construction is the inverse to the one above. Moreover, if the correspondence $\mathcal{Y}: \mathcal{H} \rightarrow \mathcal{D}$ is proper (tight) then $\mathcal{Y}: \mathcal{G} \rightarrow \mathcal{D}$ is also proper (tight).

Finally, isomorphisms of correspondences $\mathcal{H} \rightarrow \mathcal{D}$ are homeomorphisms $\mathcal{Y} \rightarrow \mathcal{Y}^{\prime}$ that are equivariant with respect to the left $\mathcal{H}$ - and right $\mathcal{D}$-actions. Thus an isomorphism $\mathcal{Y}_{1} \rightarrow \mathcal{Y}_{2}$ is also equivariant with respect to the left $\mathcal{G}$-actions, and it intertwines the isomorphisms $\varphi_{p}$ and $\varphi_{p}^{\prime}$ by Equation (3.17). Equivariance with respect to the left $\mathcal{G}$-actions means that it is an isomorphism between correspondences $\mathcal{G} \rightarrow \mathcal{D}$, and intertwining the maps $\varphi_{p}$ and $\varphi_{p}^{\prime}$ means that it is a modification $\left(\mathcal{Y}, \varphi_{p}\right) \rightarrow\left(\mathcal{Y}^{\prime}, \varphi_{p}^{\prime}\right)$.

We have shown that

$$
\begin{aligned}
\mathfrak{G r}(\mathcal{H}, \mathcal{D}) & \cong \mathfrak{G r}^{P}\left(\left(\mathcal{X}_{p}, \sigma_{p, q}\right), \text { const }_{\mathcal{D}}\right), \\
\mathfrak{G r}_{\text {prop }}(\mathcal{H}, \mathcal{D}) & \cong \mathfrak{G r}_{\text {prop }}^{P}\left(\left(\mathcal{X}_{p}, \sigma_{p, q}\right), \text { const }_{\mathcal{D}}\right), \\
\mathfrak{G r}_{\text {tight }}(\mathcal{H}, \mathcal{D}) & \cong \mathfrak{G r}_{\text {tight }}^{P}\left(\left(\mathcal{X}_{p}, \sigma_{p, q}\right), \text { const }_{\mathcal{D}}\right)
\end{aligned}
$$

Thus $\mathcal{H}$ is the colimit of the diagram $\left(\mathcal{X}_{p}, \sigma_{p, q}\right)$ in $\mathfrak{G r}, \mathfrak{G r} \mathfrak{r}_{\text {prop }}$ and $\mathfrak{G r}_{\text {tight }}$.

\subsubsection{Properties of the colimit groupoid}

We study some properties of the colimit groupoid of an Ore monoid action by tight correspondences. As before, let $P$ be an Ore monoid and let $\left(\mathcal{X}_{p}, \sigma_{p, q}\right)$ be an action of $P$ on a groupoid $\mathcal{G}$ by tight correspondences. Let $\mathcal{H}$ be the colimit of 
this action in $\mathfrak{G r}$. We will provide sufficient criteria for $\mathcal{H}$ to be Hausdorff and to posses a grading over the group completion $G=G[P]$ of $P$. We recall that $\mathcal{H}$ has a decomposition $\mathcal{H}=\bigsqcup_{g \in G} \mathcal{H}_{g}$, see Equation (3.8).

Proposition 3.16. The decomposition $\mathcal{H}=\bigsqcup_{g \in G} \mathcal{H}_{g}$ satisfies $\mathcal{H}_{g} \cdot \mathcal{H}_{h} \subseteq \mathcal{H}_{g h}$ and $\mathcal{H}_{g}^{-1}=\mathcal{H}_{g^{-1}}$. Assume that $s_{p}$ is surjective for all $p \in P$. Then $\mathcal{H}_{g} \cdot \mathcal{H}_{h}=\mathcal{H}_{g h}$ for all $g, h \in G$.

Proof. Let $g, h \in G$ and let $\left(p_{1}, q_{1}\right) \in \mathcal{C}_{P}^{g},\left(p_{2}, q_{2}\right) \in \mathcal{C}_{p}^{h}$. Since $P$ is an Ore monoid, there are $a, b \in P$ such that $q_{1} a=p_{2} b$. Furthermore, $\left(p_{1}, q_{1}\right)\left(p_{2}, q_{2}\right)=$ $\left(p_{1} a, q_{1} a\right)\left(p_{2} b, q_{2} b\right)=\left(p_{1} a, q_{2} b\right) \in \mathcal{C}_{P}^{g h}$. Now let $\left[x_{p_{1}}, x_{q_{1}}\right] \in f_{p_{1}, q_{1}}\left(\mathcal{X}_{p_{1}} \circ_{\mathcal{G}} \mathcal{X}_{q_{1}}^{*}\right) \subset \mathcal{H}_{g}$ and $\left[x_{p_{2}}, x_{q_{2}}\right] \in f_{p_{2}, q_{2}}\left(\mathcal{X}_{p_{2}} \circ_{\mathcal{G}} \mathcal{X}_{q_{2}}^{*}\right) \subset \mathcal{H}_{h}$. Then

$$
\left[x_{p_{1}}, x_{q_{1}}\right] \cdot\left[y_{p_{2}}, y_{q_{2}}\right]=\left[x_{p_{1}} \cdot x_{a} \cdot\left\langle x_{q_{1}} \cdot x_{a}, y_{p_{2}} \cdot y_{b}\right\rangle, y_{q_{2}} \cdot y_{b}\right]
$$

by (3.11). Thus $\left[x_{p_{1}}, x_{q_{1}}\right] \cdot\left[y_{p_{2}}, y_{q_{2}}\right] \in \mathcal{H}_{g h}$. Moreover, if $\left[x_{p}, x_{q}\right] \in \mathcal{H}_{g}$ then $\left[x_{p}, x_{q}\right]^{-1}=\left[x_{q}, x_{p}\right] \in \mathcal{H}_{g^{-1}}$. So $\mathcal{H}_{g}^{-1}=\mathcal{H}_{g^{-1}}$.

We assume now that the maps $s_{p}$ are all surjective. Let $g, h \in G$. Applying the Ore condition (Ore1), we may assume without loss of generality that $g h=$ $p_{1} p_{2}^{-1}, g=p_{1} p_{3}^{-1}$ and $h=p_{3} p_{2}^{-1}$ for $p_{1}, p_{2}, p_{3} \in P$. Let $p, q \in P$ with $p q^{-1}=g h$ and let $\left[x_{p}, x_{q}\right] \in \mathcal{H}_{g h}$. Then there are $k_{1}, k_{2} \in P$ with $p k_{1}=p_{1} k_{2}$ and $q k_{1}=p_{2} k_{2}$. Let $x_{k_{1}} \in \mathcal{X}_{k_{1}}$ with $r_{k_{1}}\left(x_{k_{1}}\right)=s_{p_{1}}\left(x_{p_{1}}\right)$ and let $y \in \mathcal{X}_{p_{3} k_{2}}$ be such that $s_{p_{3} k_{2}}(y)=$ $s_{k_{1}}\left(x_{k_{1}}\right)$. We have $\left[x_{p} \cdot x_{k_{1}}, y\right] \in \mathcal{H}_{g}$ and $\left[y, x_{q} \cdot x_{k_{1}}\right] \in \mathcal{H}_{h}$. Furthermore,

$$
\left[x_{p} \cdot x_{k_{1}}, y\right]\left[y, x_{q} \cdot x_{k_{1}}\right]=\left[x_{p} \cdot x_{k_{1}}, x_{q} \cdot x_{k_{1}}\right]=\left[x_{p}, x_{q}\right] .
$$

Thus $\mathcal{H}_{g h} \subseteq \mathcal{H}_{g} \cdot \mathcal{H}_{h}$ and therefore $\mathcal{H}_{g h}=\mathcal{H}_{g} \cdot \mathcal{H}_{h}$.

Remark 3.17. Proposition 3.16 shows that the map

$$
c: \mathcal{H}=\bigsqcup_{g \in G} \mathcal{H}_{g} \rightarrow G, \quad x_{g} \mapsto g,
$$

is a cocycle. Moreover, if the maps $s_{p}$ are surjective for all $p \in P$, then the decomposition $\mathcal{H}=\bigsqcup_{g \in G} \mathcal{H}_{g}$ is a $G$-grading in the sense of [10].

Lemma 3.18. With the structure maps inherited from the groupoid $\mathcal{H}$, the space $\mathcal{H}_{g}: \mathcal{H}_{1} \rightarrow \mathcal{H}_{1}$ is a partial equivalence for all $g \in G=G[P]$.

Proof. By Remark 3.14, $\mathcal{H}_{1}$ is a groupoid. Moreover, for all $\left[x_{k}, y_{k}\right] \in \mathcal{H}_{1}$ and all $\left[x_{p}, y_{q}\right] \in \mathcal{H}_{g}$ with $r\left(x_{p}\right)=r\left(y_{k}\right)$, we have

$$
\left[x_{k}, y_{k}\right] \cdot\left[x_{p}, y_{q}\right]=\left[x_{k} \cdot x_{a} \cdot\left\langle y_{k} \cdot x_{a}, x_{p} \cdot y_{b}\right\rangle, y_{q} \cdot y_{b}\right],
$$

where $a, b \in P$ are such that $k a=p b$ and $x_{a} \in \mathcal{X}_{a}, y_{b} \in \mathcal{X}_{b}$ satisfy $r\left(x_{a}\right)=s\left(x_{k}\right)$ and $r\left(y_{b}\right)=s\left(y_{q}\right)$. This implies that $\left[x_{k}, y_{k}\right] \cdot\left[x_{p}, y_{q}\right]$ is an element of $\mathcal{H}_{g}$. It follows 


\section{Actions of Ore monoids}

that the restriction of the multiplication map mult: $\mathcal{H} \times_{s, \mathcal{H}^{0}, r} \mathcal{H} \rightarrow \mathcal{H}$ to the space $\mathcal{H}_{1} \times{ }_{s, \mathcal{H}^{0}, r} \mathcal{H}_{g}$ defines a left action of the groupoid $\mathcal{H}_{1}$ on the space $\mathcal{H}_{g}$. This action is free and proper by construction. Similarly, the groupoid $\mathcal{H}_{1}$ acts freely and properly on the right on $\mathcal{H}_{g}$ and the two actions commute. Thus $\mathcal{H}_{g}: \mathcal{H}_{1} \rightarrow \mathcal{H}_{1}$ is a bicorrespondence. The source and range maps in the groupoid $\mathcal{H}$ are local homeomorphisms by Proposition 3.10. We need to show that $s_{*}: \mathcal{H}_{1} \backslash \mathcal{H}_{g} \rightarrow \mathcal{H}_{1}^{0}$ is injective. The claim regarding the range anchor map follows similarly. Let $\left[x_{p_{1}}, x_{q_{1}}\right]$ and $\left[y_{p_{2}}, y_{q_{2}}\right] \in \mathcal{H}_{g}$ with $r\left(y_{q_{2}}\right)=r\left(x_{q_{1}}\right)$. Since $P$ is an Ore monoid, there are $a, b \in P$ with $q_{1} a=q_{2} b$. Let $x_{a} \in \mathcal{X}_{a}$ and $y_{b} \in \mathcal{X}_{b}$ with $r\left(x_{a}\right)=s\left(x_{q_{1}}\right)$ and $r\left(y_{b}\right)=s\left(y_{q_{2}}\right)$. We have $\left[x_{p_{1}}, x_{q_{1}}\right]=\left[x_{p_{1}} \cdot x_{a}, x_{q_{1}} \cdot x_{a}\right]$ and $\left[y_{p_{2}}, y_{q_{2}}\right]=\left[y_{p_{2}} \cdot y_{b}, y_{q_{2}} \cdot y_{b}\right]$. Let $g:=\left\langle x_{q_{1}} \cdot x_{a}, y_{q_{2}} \cdot y_{b}\right\rangle$. Then since $p_{1} a=p_{2} b$, we have $\left[y_{p_{2}} \cdot y_{b}, x_{p_{1}} \cdot x_{a} g^{-1}\right] \in \mathcal{H}_{1}$. Moreover,

$$
\begin{aligned}
{\left[y_{p_{2}} \cdot y_{b}, x_{p_{1}} \cdot x_{a} g^{-1}\right] \cdot\left[x_{p_{1}}, x_{q_{1}}\right] } & =\left[y_{p_{2}} \cdot y_{b}, x_{p_{1}} \cdot x_{a} g^{-1}\right] \cdot\left[x_{p_{1}} \cdot x_{a}, x_{q_{1}} \cdot x_{a}\right] \\
& =\left[y_{p_{2}} \cdot y_{b}, x_{q_{1}} \cdot x_{a} g\right] \\
& =\left[y_{p_{2}} \cdot y_{b}, y_{q_{2}} \cdot y_{b}\right]=\left[y_{p_{2}}, y_{q_{2}}\right]
\end{aligned}
$$

And the claim follows.

Remark 3.19. By Lemma 3.18, the bicorrespondence $\mathcal{H}_{g}: \mathcal{H}_{1} \rightarrow \mathcal{H}_{1}$ is a groupoid equivalence $\mathcal{H}_{g}:\left.\left.\mathcal{H}_{1}\right|_{r\left(\mathcal{H}_{g}\right)} \rightarrow \mathcal{H}_{1}\right|_{s\left(\mathcal{H}_{g}\right)}$, where $\left.\mathcal{H}_{1}\right|_{r\left(\mathcal{H}_{g}\right)}$ is the restriction of the groupoid $\mathcal{H}_{1}$ to the open invariant set $r\left(\mathcal{H}_{g}\right) \subset \mathcal{H}^{0}$, and similarly for $\left.\mathcal{H}_{1}\right|_{s\left(\mathcal{H}_{g}\right)}$. Finally, we have

$$
r\left(\mathcal{H}_{g}\right)=\bigcup_{(p, q) \in \mathcal{C}_{P}^{g}} r_{q}\left(s_{q}^{-1}\left(s_{p}\left(\mathcal{X}_{p}\right)\right)\right)
$$

for all $g \in G$.

Assume now that all $s_{p}$ are surjective. Then $\mathcal{H}_{g}: \mathcal{H}_{1} \rightarrow \mathcal{H}_{1}$ is an equivalence for all $g \in G$. Let mult $_{g, h}: \mathcal{H}_{g}{ }^{\circ} \mathcal{H}^{1} \mathcal{H}_{h} \rightarrow \mathcal{H}_{g h}$ be the map induced by the product map in $\mathcal{H}$. Then mult ${ }_{g, h}$ is an isomorphism and $\left(\mathcal{H}_{g}\right.$, mult $\left._{g, h}\right)$ is an action of the group $G^{\text {op }}$ on $\mathcal{H}_{1}$ in $\mathfrak{G r}$.

Proposition 3.20. Let $\mathcal{G}$ be a groupoid and let $\left(\mathcal{X}_{p}, \sigma_{p, q}\right)$ be an action of an Ore monoid $P$ by tight correspondences. Assume that the groupoid $\mathcal{G}$ is Hausdorff and the left action of $\mathcal{G}$ on $\mathcal{X}_{p}$ is free for all $p \in P$. Then the groupoid $\mathcal{H}$ is Hausdorff.

Proof. If the groupoid $\mathcal{H}$ is not Hausdorff, then there are elements $g, h \in \mathcal{H}$ that have no disjoint neighborhoods. Since $\mathcal{H}^{0}$ is Hausdorff, this implies $s(g)=s(h)$ and $r(g)=r(h)$; otherwise we can use the range or source map to get disjoint neighborhoods for $g$ and $h$. It follows that $s(g) \in \mathcal{H}_{s(g)}^{s(g)}$ and $h^{-1} g \in \mathcal{H}_{s(g)}^{s(g)}$ have no disjoint neighborhoods. Hence in order to prove that the groupoid $\mathcal{H}$ is Hausdorff, 
it suffices to show that for every element $x \in \mathcal{H}^{0}$ and for every pair $g, h \in \mathcal{H}_{x}^{x}$ of distinct points there are disjoint open sets $U, V \subset \mathcal{H}$ such that $g$ is in $U$ and $h$ in $V$.

Now assume that the groupoid $\mathcal{G}$ is Hausdorff and that the left action $\mathcal{G}$ on $\mathcal{X}_{p}$ is free for all $p \in P$. The groupoid $\mathcal{H}$ is Hausdorff if and only if the groupoid $\mathcal{H}_{1}$ is Hausdorff. To see this, let $g \in \mathcal{G}$ and let $\left[x_{p}, x_{q}\right],\left[y_{p}, y_{q}\right] \in \mathcal{H}_{g}$ with $r_{p}\left(x_{p}\right)=$ $r_{q}\left(x_{q}\right)=r_{p}\left(y_{p}\right)=r_{q}\left(y_{q}\right)$. By Proposition 3.16 we have $\mathcal{H}_{g} \cdot \mathcal{H}_{g^{-1}} \subset \mathcal{H}_{1}$. Hence if $\mathcal{H}_{1}$ is Hausdorff, then there are disjoint open neighborhoods $U$ and $V$ for the points $\left[x_{q}, y_{q}\right]=\left[x_{p}, x_{q}\right]^{-1}\left[y_{p}, y_{q}\right]$ and $\left[y_{q}, y_{q}\right]$, respectively. The open sets $\left[x_{p}, x_{q}\right] \cdot U$ and $\left[x_{p}, x_{q}\right] \cdot V$ form disjoint neighborhoods for $\left[x_{p}, x_{q}\right],\left[y_{p}, y_{q}\right] \in \mathcal{H}_{g}$, respectively.

We will show that $\mathcal{H}_{1}$ is Hausdorff. Let $x \in \mathcal{G}^{0}$ and let $\left[x_{p}^{1}, y_{p}^{1}\right],\left[x_{p}^{2}, y_{p}^{2}\right] \in \mathcal{H}_{1}$ for $p \in P$ be two distinct points such that $r_{p}\left(x_{p}^{1}\right)=r_{p}\left(x_{p}^{2}\right)=r_{p}\left(y_{p}^{1}\right)=r_{p}\left(y_{p}^{2}\right)=x$. Since $r_{p *}$ is a homeomorphism, we have $\left[x_{p}^{1}, y_{p}^{1}\right]=\left[x_{p}, x_{p} g\right],\left[x_{p}^{2}, y_{p}^{2}\right]=\left[x_{p}, x_{p} h\right]$ for $x_{p}=x_{p}^{1}=x_{p}^{2}$ and $g, h \in \mathcal{G}_{s_{p}\left(x_{p}\right)}^{s_{p}\left(x_{p}\right)}$ with $g \neq h$. Since $\mathcal{G}$ is Hausdorff, there are disjoint open bisections $U_{1}, U_{2} \subset \mathcal{G}$ such that $g \in U_{1}, h \in U_{2}$. Let $U$ be an open bisection containing the point $x_{p}$. The multiplication map

$$
\mathcal{X}_{p} \times_{s_{p}, \mathcal{G}^{0}, r} \mathcal{G} \rightarrow \mathcal{X}_{p}, \quad\left(x_{p}, g\right) \mapsto x_{p} g,
$$

is open. Hence $U \cdot U_{i}$ is open for $i=1,2$. We set $V_{i}:=f_{p, p}\left(U \times{ }_{s_{p}, \mathcal{G}^{0}, s_{p}} U \cdot U_{i}\right)$ for $i=1,2$. Then $V_{1}, V_{2}$ are open neighborhoods of $\left(x_{p}, x_{p} g\right)$ and $\left(x_{p}, x_{p} h\right)$, respectively. This follows because the maps $f_{p, p}$ are open for all $p \in P$ by Lemma 3.9. Now assume that $V_{1} \cap V_{2} \neq \emptyset$. Then there are elements $\left(x_{p}, y_{p} \cdot g_{1}\right)$, $\left(x_{p}^{\prime}, y_{p}^{\prime} \cdot g_{2}\right) \in U \times_{s_{p}, \mathcal{G}^{0}, s_{p}} U \cdot U_{i}$ such that $f_{p, p}\left(\left[x_{p}, y_{p} \cdot g_{1}\right]\right)=f_{p, p}\left(\left[x_{p}^{\prime}, y_{p}^{\prime} \cdot g_{2}\right]\right)$. Thus $r_{p}\left(x_{p}\right)=r_{p}\left(x_{p}^{\prime}\right)$ and $r_{p}\left(y_{p}\right)=r_{p}\left(y_{p}^{\prime}\right)$ and therefore $x_{p}=x_{p}^{\prime}$ and $y_{p}=y_{p}^{\prime}$ since $r_{p}$ is a homeomorphism on the set $U$. By Lemma 3.11 the map $f_{p, p}$ is injective. So $\left[x_{p}^{\prime}, y_{p}^{\prime} \cdot g_{2}\right]=\left[x_{p}, y_{p} \cdot g_{1}\right] \in \mathcal{X}_{p} \circ_{\mathcal{G}} \mathcal{X}_{p}^{*}$. Thus $y_{p} g_{1}=y_{p} g_{2}$ and hence $g_{1}=g_{2} \in U_{1} \cap U_{2}$, which contradict our assumption that $U_{1}, U_{2}$ are disjoint. Thus $V_{1} \cap V_{2}=\emptyset$. This finishes the proof.

\subsubsection{Product systems associated with tight Ore monoid actions}

The image of the Ore monoid action $\left(\mathcal{X}_{p}, \sigma_{p, q}\right)$ under the functor $\mathfrak{G r} \rightarrow \mathfrak{C o r r}$ is a product system in Fowler's sense over the monoid $P$, see $[1, \S 3]$. In the following, we show that the groupoid constructed in Section 3.2.1 is a groupoid model for the Cuntz-Pimsner algebra of the product system constructed from the action $\left(\mathcal{X}_{p}, \sigma_{p, q}\right)$ using the functor $\mathfrak{G r} \rightarrow \mathfrak{C o r r}$. The product system associated with $\left(\mathcal{X}_{p}, \sigma_{p, q}\right)$ consists of

- the groupoid algebra $\mathrm{C}^{*}(\mathcal{G})$ as a coefficient algebra;

- correspondences $\mathrm{C}^{*}\left(\mathcal{X}_{p}\right)$ from $\mathrm{C}^{*}(\mathcal{G})$ to itself for all $p \in P \backslash\{1\}$; 
- isomorphisms of correspondences $\mu_{p, q}: \mathrm{C}^{*}\left(\mathcal{X}_{p}\right) \otimes_{\mathrm{C}^{*}(\mathcal{G})} \mathrm{C}^{*}\left(\mathcal{X}_{q}\right) \rightarrow \mathrm{C}^{*}\left(\mathcal{X}_{p q}\right)$ for all $p, q \in P \backslash\{1\}$ given by

$$
\mu_{p, q}\left(f_{1} \otimes f_{2}\right)=\left(f_{1} \otimes f_{2}\right) \circ \sigma_{p, q}
$$

for all $f_{1} \in \mathfrak{S}\left(\mathcal{X}_{p}\right)$ and $f_{2} \in \mathfrak{S}\left(\mathcal{X}_{q}\right)$. The maps $\mu_{p, q}$ satisfy the associativity condition

$$
\mu_{p, q r} \circ\left(\mathrm{id} \otimes_{A} \mu_{q, r}\right)=\mu_{p q, r} \circ\left(\mu_{p, q} \otimes_{A} \mathrm{id}\right): \mathcal{E}_{p} \otimes_{A} \mathcal{E}_{q} \otimes_{A} \mathcal{E}_{r} \rightarrow \mathcal{E}_{p q r} .
$$

This follows from the associativity condition (3.1) for $\sigma_{p, q}$.

In [2], we describe the Cuntz-Pimsner algebra for a given product system as the full sectional $\mathrm{C}^{*}$-algebra of a Fell bundle. We recall the construction of the Fell bundle from the product system $\left(\mathrm{C}^{*}\left(\mathcal{X}_{p}\right)\right)_{p \in P}$.

The isomorphism $\mu_{p, q}: \mathrm{C}^{*}\left(\mathcal{X}_{p}\right) \otimes_{A} \mathrm{C}^{*}\left(\mathcal{X}_{q}\right) \rightarrow \mathrm{C}^{*}\left(\mathcal{X}_{p q}\right)$ induces a nondegenerate *-homomorphism

$$
\varphi_{p, q}: \mathbb{K}\left(\mathrm{C}^{*}\left(\mathcal{X}_{p}\right)\right) \rightarrow \mathbb{K}\left(\mathrm{C}^{*}\left(\mathcal{X}_{p q}\right)\right), \quad T \mapsto \mu_{p, q}\left(T \otimes_{A} \operatorname{id}_{\mathrm{C}^{*}\left(\mathcal{X}_{q}\right)}\right) \mu_{p, q}^{*}
$$

The correspondence $\mathcal{X}_{p}$ is tight. Hence $\mathrm{C}^{*}\left(\mathcal{X}_{q}\right)$ is proper; $\varphi_{p, q}\left(\mathbb{K}\left(\mathrm{C}^{*}\left(\mathcal{X}_{p}\right)\right)\right)$ is contained in $\mathbb{K}\left(\mathrm{C}^{*}\left(\mathcal{X}_{p q}\right)\right)$.

For $\left(p_{1}, p_{2}\right) \in R_{g}$, let $\mathcal{O}_{p_{1}, p_{2}}:=\mathbb{K}\left(\mathrm{C}^{*}\left(\mathcal{X}_{p_{2}}\right), \mathrm{C}^{*}\left(\mathcal{X}_{p_{1}}\right)\right)$. We define a contraction

$$
\varphi_{p_{1}, p_{2}}^{h}: \mathcal{O}_{p_{1}, p_{2}} \rightarrow \mathcal{O}_{p_{1} h, p_{2} h}, \quad T \mapsto \mu_{p_{1}, h}\left(T \otimes_{A} \operatorname{id}_{\mathrm{C}^{*}\left(\mathcal{X}_{h}\right)}\right) \mu_{p_{2}, h}^{*} .
$$

The maps $\varphi_{p, q}^{h}$ form a functor from $\mathcal{C}_{P}^{g}$ to the category of Banach spaces with linear contractions. The category $\mathcal{C}_{P}^{g}$ is filtered. This allows us to take the colimit $\mathcal{O}_{g}$ of this diagram. If $g_{1}$ and $g_{2} \in G,\left(p_{1}, p_{2}\right) \in R_{g_{1}}$ and $\left(p_{2}, p_{3}\right) \in R_{g_{1}}$, then $\left(p_{1}, p_{3}\right) \in R_{g_{1} g_{2}}$. The composition of compact operators gives a bounded bilinear map $\mathcal{O}_{p_{1}, p_{2}} \times \mathcal{O}_{p_{2}, p_{3}} \rightarrow \mathcal{O}_{p_{1}, p_{3}}$. These maps induce a bounded linear map

$$
\mathcal{O}_{g_{1}} \times \mathcal{O}_{g_{2}} \rightarrow \mathcal{O}_{g_{3}}
$$

In addition, the adjoint maps $\mathcal{O}_{p_{1}, p_{2}} \rightarrow \mathcal{O}_{p_{2}, p_{1}}, T \mapsto T^{*}$ induce an involution $\mathcal{O}_{g} \rightarrow$ $\mathcal{O}_{g^{-1}}$. The multiplication maps and the involutions on $\left(\mathcal{O}_{g}\right)_{g \in G}$ give a Fell bundle over the group $G$. By [1, Theorem 3.16], the Cuntz-Pimsner algebra of the product system $\left(\mathrm{C}^{*}(\mathcal{G}), \mathrm{C}^{*}\left(\mathcal{X}_{p}\right), \mu_{p, q}\right)$ is isomorphic to the full sectional $\mathrm{C}^{*}$-algebra of the Fell bundle $\left(\mathcal{O}_{g}\right)_{g \in G}$.

Theorem 3.21. The groupoid $\mathrm{C}^{*}$-algebra $\mathrm{C}^{*}(\mathcal{H})$ is canonically isomorphic to the Cuntz-Pimsner algebra of the product system $\left(\mathrm{C}^{*}\left(\mathcal{X}_{p}\right)\right)_{p \in P}$ over $P$ described above. 
Proof. For $p, q \in P$, let $\psi_{p, q}: \mathfrak{S}\left(\mathcal{X}_{p} \circ_{\mathcal{G}} \mathcal{X}_{q}^{*}\right) \rightarrow \mathbb{K}\left(\mathrm{C}^{*}\left(\mathcal{X}_{q}\right), \mathrm{C}^{*}\left(\mathcal{X}_{p}\right)\right)$ be the map given by

$$
\psi_{p, q}(f)(\xi)\left(x_{p}\right):=\sum_{x_{q} \in \mathcal{X}_{q}: s\left(x_{p}\right)=s\left(x_{q}\right)} f\left(\left[x_{p}, x_{q}\right]\right) \xi\left(x_{q}\right)
$$

for $f \in \mathfrak{S}\left(\mathcal{X}_{p} \circ_{\mathcal{G}} \mathcal{X}_{q}^{*}\right), \xi \in \mathrm{C}^{*}\left(\mathcal{X}_{p}\right)$ and $x_{p} \in \mathcal{X}_{p}$. We claim that $\psi$ is an injective *-homomorphism.

Let $\xi \in \mathfrak{S}\left(\mathcal{X}_{p}\right), \eta \in \mathfrak{S}\left(\mathcal{X}_{q}\right)$. Then the function $\theta_{\xi, \eta} \in \mathfrak{S}\left(\mathcal{X}_{p} \circ_{\mathcal{G}} \mathcal{X}_{q}^{*}\right)$ given by

$$
\theta_{\xi, \eta}\left(\left[x_{p}, x_{q}\right]\right):=\sum_{g \in \mathcal{G}: r(g)=s\left(x_{q}\right)} \xi\left(x_{p} \cdot g\right) \cdot \overline{\eta\left(x_{q} \cdot g\right)}
$$

satisfies

$$
\begin{aligned}
\psi_{p, q}\left(\theta_{\xi, \eta}\right)(\zeta)\left(x_{p}\right) & =\sum_{x_{q} \in \mathcal{X}_{q}: s_{p}\left(x_{p}\right)=s_{q}\left(x_{q}\right)} \zeta\left(x_{q}\right) \sum_{g \in \mathcal{G}: r(g)=s\left(x_{q}\right)} \xi\left(x_{p} \cdot g\right) \cdot \overline{\eta\left(x_{q} \cdot g\right)} \\
& =\sum_{g \in \mathcal{G}: r(g)=s\left(x_{p}\right)} \xi\left(x_{p} \cdot g\right) \sum_{x_{q} \in \mathcal{X}_{q}: s(g)=s\left(x_{q}\right)} \overline{\eta\left(x_{q}\right)} \zeta\left(x_{q} \cdot g^{-1}\right) \\
& =\sum_{g \in \mathcal{G}: r(g)=s\left(x_{p}\right)} \xi\left(x_{p} \cdot g\right)\langle\eta, \zeta\rangle\left(g^{-1}\right) \\
& =\xi \cdot\langle\eta, \zeta\rangle\left(x_{p}\right) \\
& =|\xi\rangle\langle\eta|(\zeta)\left(x_{p}\right)
\end{aligned}
$$

for all $x_{p} \in \mathcal{X}_{p}$. Thus $\psi_{p, q}\left(\theta_{\xi, \eta}\right)=|\xi\rangle\langle\eta| \in \mathbb{K}\left(\mathrm{C}^{*}\left(\mathcal{X}_{q}\right), \mathrm{C}^{*}\left(\mathcal{X}_{p}\right)\right)$. Let $f \in \mathfrak{S}\left(\mathcal{X}_{p} \circ \mathcal{G} \mathcal{X}_{q}^{*}\right)$ be such that $\psi_{p, q}(f)=0$. Then

$$
\psi_{p, q}(f)(\xi)\left(x_{p}\right)=\sum_{x_{q} \in \mathcal{X}_{q}: s\left(x_{p}\right)=s\left(x_{q}\right)} \xi\left(x_{q}\right) f\left(x_{p}, x_{q}\right)=0
$$

for all $\xi \in \mathrm{C}^{*}\left(\mathcal{X}_{p}\right)$ and $x_{p} \in \mathcal{X}_{p}$. In particular, the equality holds for the functions $f_{x_{p}} \in \mathrm{C}^{*}\left(\mathcal{X}_{p}\right)$ given by $f_{x_{p}}\left(x_{q}\right):=\overline{f\left(x_{p}, x_{q}\right)}$ for all $x_{q} \in \mathcal{X}_{q}, x_{p} \in \mathcal{X}_{q}$ with $s\left(x_{p}\right)=s\left(x_{q}\right)$. Thus

$$
\psi_{p, q}(f)\left(f_{x_{p}}\right)\left(x_{p}\right)=\sum_{x_{q} \in \mathcal{X}_{q}: s\left(x_{p}\right)=s\left(x_{q}\right)} \overline{f\left(x_{p}, x_{q}\right)} f\left(x_{p}, x_{q}\right)=0 .
$$

Hence $f\left(x_{p}, x_{q}\right)=0$ for all $\left(x_{p}, x_{q}\right) \in \mathcal{X}_{p} \times_{s_{p}, \mathcal{G}^{0}, s_{q}} \mathcal{X}_{q}^{*}$. Thus $\psi_{p, q}$ is injective from $\mathcal{X}_{p} \circ \mathcal{G} \mathcal{X}_{q}^{*}$ to $\mathbb{K}\left(\mathrm{C}^{*}\left(\mathcal{X}_{q}\right), \mathrm{C}^{*}\left(\mathcal{X}_{p}\right)\right)$. We have related compact operators $\mathrm{C}^{*}\left(\mathcal{X}_{q}\right) \rightarrow$ $\mathrm{C}^{*}\left(\mathcal{X}_{p}\right)$ to kernel functions, getting continuous linear maps

$$
K_{p, q}:=\mathfrak{S}\left(\mathcal{X}_{p} \circ_{\mathcal{G}} \mathcal{X}_{q}^{*}\right) \subseteq \mathbb{K}\left(\mathrm{C}^{*}\left(\mathcal{X}_{q}\right), \mathrm{C}^{*}\left(\mathcal{X}_{p}\right)\right)
$$




\section{Actions of Ore monoids}

Let $f_{1} \in \mathfrak{S}\left(\mathcal{X}_{p} \circ_{\mathcal{G}} \mathcal{X}_{q}^{*}\right)$ and $f_{2} \in \mathfrak{S}\left(\mathcal{X}_{q} \circ_{\mathcal{G}} \mathcal{X}_{k}^{*}\right)$ for $p, q, k \in P$. Then the convolution product $f_{1} \star f_{2} \in \mathfrak{S}(\mathcal{H})$ is an element in $\mathfrak{S}\left(\mathcal{X}_{p} \circ \mathcal{G} \mathcal{X}_{k}^{*}\right)$; it is given by

$$
f_{1} \star f_{2}\left(x_{p}, x_{k}\right)=\sum_{x_{q} \in \mathcal{X}_{q}: s\left(x_{q}\right)=s\left(x_{k}\right)} f_{1}\left(x_{p}, x_{q}\right) f_{2}\left(x_{q}, x_{k}\right) .
$$

Furthermore,

$$
\begin{aligned}
\psi_{p, k}\left(f_{1} \star f_{2}\right)(\xi)\left(x_{p}\right) & =\sum_{x_{k} \in \mathcal{X}_{k}: s\left(x_{k}\right)=s\left(x_{p}\right)} \xi\left(x_{k}\right) \sum_{x_{q} \in \mathcal{X}_{q}: s\left(x_{q}\right)=s\left(x_{p}\right)} f_{1}\left(x_{p}, x_{q}\right) f_{2}\left(x_{q}, x_{k}\right) \\
& =\sum_{x_{k} \in \mathcal{X}_{k}: s\left(x_{k}\right)=s\left(x_{p}\right)} \sum_{x_{q} \in \mathcal{X}_{q}: s\left(x_{q}\right)=s\left(x_{p}\right)} \xi\left(x_{k}\right) f_{1}\left(x_{p}, x_{q}\right) f_{2}\left(x_{q}, x_{k}\right) \\
& =\psi_{p, q}\left(f_{1}\right)\left(\psi_{q, k}\left(f_{2}\right)(\xi)\right)\left(x_{p}\right)
\end{aligned}
$$

for all $\xi \in \mathfrak{S}\left(\mathcal{X}_{k}\right)$ and all $x_{p} \in \mathcal{X}_{p}$. Let $\xi \in \mathfrak{S}\left(\mathcal{X}_{p}\right), \eta \in \mathfrak{S}\left(\mathcal{X}_{q}\right)$. Then $\theta_{\eta, \xi}^{*} \in \mathfrak{S}(\mathcal{H})$ is supported on the set $\mathcal{X}_{q} \circ_{\mathcal{G}} \mathcal{X}_{p}^{*}$. It is given by

$$
\theta_{\eta, \xi}^{*}\left(x_{q}, x_{p}\right)=\overline{\sum_{g \in \mathcal{G}: r(g)=s\left(x_{q}\right)} \eta\left(x_{p} \cdot g\right) \cdot \overline{\xi\left(x_{q} \cdot g\right)}}=\theta_{\xi, \eta}\left(x_{p}, x_{q}\right) .
$$

Hence

$$
\psi_{p, q}\left(\theta_{\eta, \xi}^{*}\right)=\psi_{p, q}\left(\theta_{\xi, \eta}\right)=|\xi\rangle\langle\eta|=| \eta\rangle\left\langle\left.\xi\right|^{*}=\psi_{q, p}\left(\theta_{\eta, \xi}\right)^{*}\right.
$$

We have proved that the convolution on $\mathfrak{S}\left(\mathcal{H}^{1}\right)$ restricted to $K_{p_{2}, p_{3}} \otimes K_{p_{1}, p_{2}}$ for $p_{1}, p_{2}, p_{3} \in P$ is a map to $K_{p_{1}, p_{3}}$; the description of the composition of compact operators $\mathrm{C}^{*}\left(\mathcal{X}_{p_{1}}\right) \rightarrow \mathrm{C}^{*}\left(\mathcal{X}_{p_{2}}\right) \rightarrow \mathrm{C}^{*}\left(\mathcal{X}_{p_{3}}\right)$ through kernel functions shows that the convolution on $\mathfrak{S}\left(H_{1}\right)$ and the composition of compact operators both restrict to the same map $K_{p_{2}, p_{3}} \otimes K_{p_{1}, p_{2}} \rightarrow K_{p_{1}, p_{3}}$. Furthermore, the involution on $\mathfrak{S}\left(\mathcal{H}^{1}\right)$ and the involution $\mathbb{K}\left(\mathrm{C}^{*}\left(\mathcal{X}_{p_{2}}\right), \mathrm{C}^{*}\left(\mathcal{X}_{p_{1}}\right)\right) \rightarrow \mathbb{K}\left(\mathrm{C}^{*}\left(\mathcal{X}_{p_{1}}\right), \mathrm{C}^{*}\left(\mathcal{X}_{p_{2}}\right)\right)$ restrict to the same $\operatorname{map} K_{p_{1}, p_{2}} \rightarrow K_{p_{2}, p_{1}}$.

Next let $p, q, k \in P$. Equation (3.5) gives a map $\alpha_{p, q}^{k}: \mathcal{X}_{p} \circ_{\mathcal{G}} \mathcal{X}_{q}^{*} \rightarrow \mathcal{X}_{p k} \circ_{\mathcal{G}} \mathcal{X}_{q k}^{*}$. It induces a map $\left(\alpha_{p, q}^{k}\right)_{*}: K_{p, q} \rightarrow K_{p k, q k}$ by setting

$$
\left(\alpha_{p, q}^{k}\right)_{*}(\psi)\left[x_{p k}, x_{q k}\right]=\sum_{\substack{\left[x_{p}, x_{k}\right] \in \mathcal{X}_{p} \circ \mathcal{G} \mathcal{X}_{q}^{*} \\ \alpha_{p, q}^{k}\left(\left[x_{p}, x_{k}\right]\right)=\left[x_{p k}, x_{q k}\right]}} \psi\left(\left[x_{p}, x_{k}\right]\right) .
$$

The following diagram commutes:

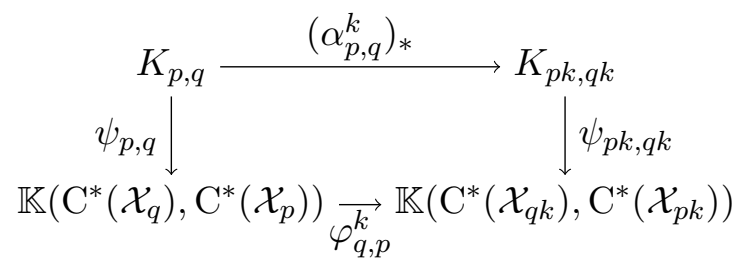


Now let $p \in P$. The space $\mathcal{X}_{p} \circ \mathcal{X}_{p}^{*}$ is a subgroupoid of the groupoid $\mathcal{H}$. To see this, let $\left[x_{p}, y_{p}\right]$ and $\left[m_{p}, n_{p}\right]$ in $\mathcal{X}_{p} \circ \mathcal{X}_{p}^{*}$ with $r_{p}\left(m_{p}\right)=r_{p}\left(y_{p}\right)$. Then

$$
\left[x_{p}, y_{p}\right] \cdot\left[m_{p}, n_{p}\right]=\left[x_{p}\left\langle y_{p}, m_{p}\right\rangle, n_{p}\right],
$$

which belongs to $\mathcal{X}_{p} \circ \mathcal{X}_{p}^{*}$. Recall that $\left\langle y_{p}, m_{p}\right\rangle$ is the unique point in $\mathcal{G}$ with $y_{p} g=$ $m_{p}$. Moreover,

$$
\left[x_{p}, y_{p}\right]^{-1}=\left[y_{p}, x_{p}\right]
$$

for all $\left[x_{p}, y_{p}\right] \in \mathcal{X}_{p} \circ \mathcal{X}_{p}^{*}$. Finally, the unit space of the groupoid $\mathcal{X}_{p} \circ \mathcal{X}_{p}^{*}$ is given by

$$
\left(\mathcal{X}_{p} \circ \mathcal{X}_{p}^{*}\right)^{0}=\left\{\left[x_{p}, x_{p}\right] \in \mathcal{X}_{p} \circ \mathcal{X}_{p}^{*}\right\} .
$$

Therefore, the map

$$
\mathcal{X}_{p} \circ \mathcal{X}_{p}^{*} \rightarrow \mathcal{X}_{p} / \mathcal{G}, \quad\left[x_{p}, x_{p}\right] \mapsto\left[x_{p}\right],
$$

is a homeomorphism. The ${ }^{*}$-algebra structure on $K_{p, p}$ comes also from the structure of $\mathcal{X}_{p} \circ \mathcal{X}_{p}^{*}$. The $\mathrm{C}^{*}$-completion $\mathrm{C}^{*}\left(K_{p, p}\right)$ of $K_{p, p}$ is the groupoid algebra $\mathrm{C}^{*}\left(\mathcal{X}_{p} \circ\right.$ $\left.\mathcal{X}_{p}^{*}\right)$. The space $\mathcal{X}_{p}$ induces a Morita equivalence between the groupoid $\mathcal{X}_{p} \circ \mathcal{X}_{p}^{*}$ and $\left.\mathcal{G}\right|_{s_{p}\left(\mathcal{X}_{p}\right)}$, see $\left[34\right.$, p. 5]. Hence $\mathrm{C}^{*}\left(K_{p, p}\right)=\mathrm{C}^{*}\left(\mathcal{X}_{p} \circ \mathcal{X}_{p}^{*}\right)$ is Morita-Rieffel equivalent to $\mathrm{C}^{*}\left(\left.\mathcal{G}\right|_{s_{p}\left(\mathcal{X}_{p}\right)}\right)$. Thus the inclusion map $K_{p, p} \rightarrow \mathbb{K}\left(\mathrm{C}^{*}\left(\mathcal{X}_{p}\right)\right)$ extends to an isomorphism

$$
\mathrm{C}^{*}\left(K_{p, p}\right) \rightarrow \mathbb{K}\left(\mathrm{C}^{*}\left(\mathcal{X}_{p}\right)\right) .
$$

This implies that the inclusion $K_{p, p} \rightarrow \mathfrak{S}\left(H_{1}\right) \subseteq \mathrm{C}^{*}(H)$ gives a ${ }^{*}$-homomorphism $\mathbb{K}\left(\mathrm{C}^{*}\left(\mathcal{X}_{p}\right)\right) \rightarrow \mathrm{C}^{*}(H)$. In addition,

$$
\mathcal{H}_{1}=\lim _{\longrightarrow} \mathcal{X}_{p} \circ \mathcal{G} \mathcal{X}_{p}^{*}
$$

implies

$$
\mathfrak{S}\left(\mathcal{H}_{1}\right)=\lim _{\longrightarrow} \mathfrak{S}\left(\mathcal{X}_{p} \circ_{\mathcal{G}} \mathcal{X}_{p}^{*}\right) .
$$

Moreover, a $\mathrm{C}^{*}$-seminorm on $\mathfrak{S}\left(\mathcal{H}_{1}\right)$ which is continuous with respect to the inductive limit topology is the same as a family of $\mathrm{C}^{*}$-seminorms on $\mathfrak{S}\left(\mathcal{X}_{p} \circ_{\mathcal{G}} \mathcal{X}_{p}^{*}\right)$ each continuous with respect to the inductive limit topology. Thus

$$
\mathrm{C}^{*}\left(\mathcal{H}_{1}\right)=\underline{\lim _{\mathrm{W}}} \mathrm{C}^{*}\left(\mathcal{X}_{p} \circ_{\mathcal{G}} \mathcal{X}_{p}^{*}\right)=\underline{\lim } \mathbb{K}\left(\mathrm{C}^{*}\left(\mathcal{X}_{p}\right)\right)=\mathcal{O}_{1} .
$$

By the results of [10], the $\mathrm{C}^{*}$-algebra $\mathrm{C}^{*}(\mathcal{H})$ is the sectional $\mathrm{C}^{*}$-algebra of

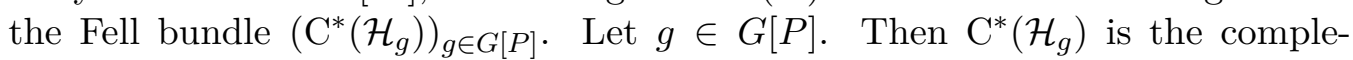
tion of $\mathfrak{S}\left(\mathcal{H}_{g}\right)$ in the norm obtained from the right $\mathrm{C}^{*}\left(\mathcal{H}_{1}\right)$-valued inner product. By [1, Theorem 3.15], the Cuntz-Pimsner algebra $\mathcal{O}$ of the product system $\left(\mathrm{C}^{*}\left(\mathcal{X}_{p}\right)\right)_{p \in P}$ is the sectional $\mathrm{C}^{*}$-algebra of the Fell bundle $\left(\mathcal{O}_{g}\right)_{g \in G[P]}$, where $\mathcal{O}_{g}=\lim _{(p, q) \in \mathcal{C}_{P}^{g}} \mathbb{K}\left(\mathrm{C}^{*}\left(\mathcal{X}_{q}\right), \mathrm{C}^{*}\left(\mathcal{X}_{p}\right)\right)$. Here $\mathcal{O}_{g}$ is completed with respect to the 
right $\mathcal{O}_{1}$-valued inner product. Since $\mathfrak{S}\left(\mathcal{H}_{g}\right)=\lim _{(p, q) \in \mathcal{C}_{P}^{g}} \mathfrak{S}\left(\mathcal{X}_{p} \circ \mathcal{G} \mathcal{X}_{q}^{*}\right)$ is a dense subspace in $\mathcal{O}_{g}$, it follows that $\mathcal{O}_{g}$ is isomorphic to $\mathcal{H}_{g}$. Thus the Fell bundles $\left(\mathrm{C}^{*}\left(\mathcal{H}_{g}\right)\right)_{g \in G[P]}$ and $\left(\mathcal{O}_{g}\right)_{g \in G[P]}$ are isomorphic. So $\mathcal{O}$ is isomorphic to $\mathrm{C}^{*}(\mathcal{H})$.

\subsection{Actions of Ore monoids by proper correspondences}

In this section, we study Ore monoid actions in the bicategory of proper correspondences $\mathfrak{G r}_{\text {prop }}$. We show two main results. First, every diagram of Ore shape in $\mathfrak{G r}_{\text {prop }}$ has a colimit. Secondly, the colimit of a diagram of Ore shape is a groupoid model of the Cuntz-Pimsner algebra of the associated product system.

To construct the colimit of an Ore monoid action in $\mathfrak{G r}_{\text {prop }}$, we provide a colimit preserving map from $\mathfrak{G} \mathfrak{r}_{\text {prop }}^{P}$ to $\mathfrak{G} \mathfrak{r}_{\text {tight }}^{P}$ for a given monoid $P$. This allows us to apply the construction in Section 3.2 to obtain a groupoid colimit of a given action by proper correspondences. We also show that the map $\mathfrak{G r}_{\text {prop }}^{P} \rightarrow \mathfrak{G r}_{\text {tight }}^{P}$ induces a colimit preserving map on the $\mathrm{C}^{*}$-algebraic level. This gives a concrete groupoid model for the Cuntz-Pimsner algebra of the product system associated with an Ore monoid action in $\mathfrak{G r}_{\text {prop }}$.

\subsubsection{Tightening a proper Ore monoid action}

In this section, we fix a groupoid $\mathcal{G}$, an Ore monoid $P$ and an action $\left(\mathcal{X}_{p}, \sigma_{p, q}\right)$ of $P$ on $\mathcal{G}$ by proper correspondences. As a preparation, we start with some results about proper correspondences.

Lemma 3.22. Let $\mathcal{D}$ be a groupoid and let $\mathcal{X}, \mathcal{Y}$ be proper $\mathcal{D}$-correspondences. Then the map $r_{\mathcal{X}, \mathcal{Y}}: \mathcal{X} \circ \mathcal{Y} / \mathcal{D} \rightarrow \mathcal{X} / \mathcal{D}$ given by

$$
r_{\mathcal{X}, \mathcal{Y}}[(x, y)]:=[x]
$$

is proper.

Proof. The correspondence $\mathcal{X} \circ \mathcal{Y}$ is proper by Proposition 2.27. That is, the map induced by the range map $r_{\mathcal{X}} \mathcal{Y}_{*}: \mathcal{X} \circ \mathcal{Y} / \mathcal{D} \rightarrow \mathcal{D}^{0}$ is proper. We have $r_{\mathcal{X}} \circ \mathcal{Y}_{*}=$ $r_{\mathcal{X} *} \circ r_{\mathcal{X}, \mathcal{Y}}$. It follows that $r_{\mathcal{X}, \mathcal{Y}}$ is a proper map since $\mathcal{X} \circ \mathcal{Y} / \mathcal{D}$ is Hausdorff and $r_{\mathcal{X} *}$ is proper and continuous.

Let $\mathcal{D}$ be a groupoid and let $\mathcal{X}$ and $\mathcal{Y}$ be proper $\mathcal{D}$-correspondences. For $x \in \mathcal{X}$ we let

$$
C_{x}:=\left\{z \in \mathcal{X} \circ \mathcal{Y} \mid r_{\mathcal{X}, \mathcal{Y}}([z])=[x]\right\} .
$$

Let $C_{x}^{\prime}:=\mathrm{p}^{-1}\left(C_{x}\right) \subset \mathcal{X} \times_{s, \mathcal{D}^{0}, r} \mathcal{Y}$, where $\mathrm{p}: \mathcal{X} \times_{s, \mathcal{D}^{0}, r} \mathcal{Y} \rightarrow \mathcal{X} \circ \mathcal{Y}$ be the orbit space projection. 
Lemma 3.23. Let $\mathcal{D}$ be a groupoid and let $\mathcal{X}$ and $\mathcal{Y}$ be proper $\mathcal{D}$-correspondences. For all $x \in \mathcal{X}$, the restriction of the quotient map $\mathrm{p}: \mathcal{X} \times_{s, \mathcal{D}^{0}, r} \mathcal{Y} \rightarrow \mathcal{X} \circ \mathcal{Y}$ to $C_{x}^{\prime}$ is injective.

Proof. Let $\left(x, \xi_{1}\right),\left(x, \xi_{2}\right) \in \mathcal{X} \times_{\mathcal{G}^{0}} \mathcal{Y}$ be such that $\mathrm{p}\left(x, \xi_{1}\right)=\mathrm{p}\left(x, \xi_{2}\right)$. It follows that there is an element $g \in \mathcal{D}_{s(x)}$ such that $\left(x \cdot g, g^{-1} \cdot \xi_{1}\right)=\left(x, \xi_{2}\right)$. Hence $x \cdot g=x$ and $g^{-1} \cdot \xi_{1}=\xi_{2}$. By our assumption, the right action of $\mathcal{D}$ on $\mathcal{X}$ is free. This implies $g=s(x)$ and hence $\xi_{1}=\xi_{2}$.

Finally, let $S_{x, \mathcal{Y}}: C_{x} \subset \mathcal{X} \circ \mathcal{Y} \rightarrow \mathcal{Y}$ be the map given by

$$
S_{x, \mathcal{Y}}(\xi):=\mathrm{pr}_{2} \circ \mathrm{p}^{-1}(\xi)
$$

for all $\xi \in C_{x}$, where $\operatorname{pr}_{2}: \mathcal{X} \times_{s, \mathcal{D}^{0}, r} \mathcal{Y} \rightarrow \mathcal{Y}$ is the projection map.

Corollary 3.24. Let $\mathcal{D}$ be a groupoid and let $\mathcal{X}: \mathcal{D} \rightarrow \mathcal{D}$ and $\mathcal{Y}: \mathcal{D} \rightarrow \mathcal{D}$ be proper correspondences. For all $x \in \mathcal{X}$, the map $S_{x, \mathcal{Y}}: C_{x} \rightarrow \mathcal{Y}$ is injective and continuous.

Proof. The map $S_{x, y}$ is continuous since it is the composition of two continuous maps. It is injective by Lemma 3.23.

Now we turn back to our monoid action $\left(\mathcal{X}_{p}, \sigma_{p, q}\right)$. The isomorphisms $\sigma_{p, q}$ induce homeomorphisms $\sigma_{p, q_{*}}: \mathcal{X}_{p q} / \mathcal{G} \rightarrow \mathcal{X}_{p} \circ_{\mathcal{G}} \mathcal{X}_{q} / \mathcal{G}$. We abbreviate

$$
r_{p, q}:=r_{\mathcal{X}_{p}, \mathcal{X}_{q}} \circ \sigma_{p, q_{*}}: \mathcal{X}_{p q} / \mathcal{G} \rightarrow \mathcal{X}_{p} / \mathcal{G}
$$

for all $p, q \in P$. Lemma 3.22 implies that $r_{p, q}$ is proper for all $p$ and $q \in P$. Notice that $r_{1, p}$ is the map induced by the range map $r_{\mathcal{X}_{p}}$ on the quotient space $\mathcal{X}_{p} / \mathcal{G}$ by our assumption that $\mathcal{X}_{1}$ is the identity correspondence $\mathcal{G}: \mathcal{G} \rightarrow \mathcal{G}$. Moreover, since the maps $\sigma_{p, q}$ satisfy (3.1) we obtain

$$
r_{p, q} r_{p q, t}=r_{p, q t}
$$

for all $p, q, t \in P$. Thus $\left(\mathcal{X}_{p} / \mathcal{G}, r_{p, q}\right)$ is a projective system over the directed category $\mathcal{C}_{P}$ (see Definition 3.1 ). We denote its projective limit by

$$
\mathcal{H}^{0}:=\lim _{\overleftarrow{\mathcal{C}}_{P}}\left(\mathcal{X}_{p} / \mathcal{G}, r_{p, q}\right)
$$

A point $\eta \in \mathcal{H}^{0}$ is given by $\left(\eta_{p}\right)_{p \in P}$ with $r_{p, q}\left(\eta_{p q}\right)=\eta_{p}$ for all $p, q \in P$.

Lemma 3.25. The space $\mathcal{H}^{0}$ is locally compact and Hausdorff. The maps

$$
\pi_{p}: \mathcal{H}^{0} \rightarrow \mathcal{X}_{p} / \mathcal{G}, \quad\left(\eta_{p}\right)_{p \in P} \mapsto \eta_{p},
$$

are proper for all $p \in P$. 


\section{Actions of Ore monoids}

Proof. By Proposition 2.17, the spaces $\mathcal{X}_{p} / \mathcal{G}$ are locally compact Hausdorff for all $p \in P$. The maps $r_{p, q}$ are proper for all $p, q \in P$. The proof then follows using similar arguments as in [1, Lemma 4.9].

There is a map $r: \mathcal{H}^{0} \rightarrow \mathcal{G}^{0}$ given by

$$
r(\eta):=r_{1,1}\left(\eta_{1}\right)
$$

for all $\eta=\left(\eta_{p}\right)_{p \in P} \in \mathcal{H}^{0}$. The associativity condition (3.28) implies that $r(\eta)=$ $\left(r_{\mathcal{X}_{p}}\right)_{*}\left(\eta_{p}\right)$ for all $p \in P$. Moreover, Lemma 3.25 implies that $r: \mathcal{H}^{0} \rightarrow \mathcal{G}^{0}$ is a proper map; it is the anchor map of a natural action of $\mathcal{G}$ on $\mathcal{H}^{0}$. It is given by

$$
g \cdot\left(\eta_{p}\right)_{p \in P}=\left(g \eta_{p}\right)_{p \in P}
$$

for all $g \in \mathcal{G}$ and all $\eta=\left(\eta_{p}\right)_{p \in P} \in \mathcal{H}^{0}$ with $r(\eta)=s(g)$. Let

$$
\tilde{\mathcal{G}}:=\mathcal{G} \ltimes \mathcal{H}^{0}
$$

be the transformation groupoid associated with this action. Let

$$
\tilde{\mathcal{X}}_{p}:=\mathcal{X}_{p} \times_{s_{p}, \mathcal{G}^{0}, r} \mathcal{H}^{0}
$$

for all $p \in P$. We claim that $\tilde{\mathcal{X}}_{p}$ is naturally a tight correspondence over the groupoid $\tilde{\mathcal{G}}$. First, we define the anchor maps $\tilde{r}_{p}, \tilde{s}_{p}: \tilde{\mathcal{G}} \rightarrow \mathcal{H}^{0}=\tilde{\mathcal{G}}^{0}$. Given a point $\eta \in \mathcal{H}^{0}$ and an element $x_{p} \in \mathcal{X}_{p}$ such that $r(\eta)=s_{p}\left(x_{p}\right)$, we may concatenate $x_{p}$ to $\eta$ and get a new point $x_{p} \cdot \eta \in \mathcal{H}^{0}$, defined formally by

$$
\left(x_{p} \cdot \eta\right)_{p t}:=\mathrm{p}_{p t} \circ \sigma_{p, t}^{-1}\left(x_{p}, \eta_{t}^{\prime}\right)
$$

for all $t \in P$, where $\mathrm{p}_{p t}: \mathcal{X}_{p t} \rightarrow \mathcal{X}_{p t} / \mathcal{G}$ is the quotient map and $\eta_{t}^{\prime}$ is any point in $\mathcal{X}_{t}$ such that $\mathrm{p}_{t}\left(\eta_{t}^{\prime}\right)=\eta_{t}$. If $b \in P$ is not of the form $p t$ for some $t \in P$ then by the Ore condition, we may find $k_{1}$ and $k_{2} \in P$ with $b k_{1}=p k_{2}$. We define

$$
\left(x_{p} \cdot \eta\right)_{b}:=r_{b, k_{1}}\left(\left(x_{p} \cdot \eta\right)_{p k_{2}}\right) .
$$

The point $\left(x_{p} \cdot \eta\right)_{p t}$ does not depend on the choice of $\eta_{t}^{\prime}$ since $\sigma_{p, t}^{-1}$ is $\mathcal{G}$-equivariant. Here we use implicitly that because the Ore monoid $p P$ is cofinal in $P$ there is a homeomorphism

$$
\mathcal{H}^{0}=\lim _{\overleftarrow{\mathcal{C}_{P}}}\left(\mathcal{X}_{p} / \mathcal{G}, r_{p, q}\right) \rightarrow \lim _{\overleftarrow{\mathcal{C}_{p P}}}\left(\mathcal{X}_{p t} / \mathcal{G}, r_{p k, p l}\right)
$$

It follows that, for all $x_{p} \in \mathcal{X}_{p}$, we have a map

$$
\left(\mathcal{H}^{0}\right)^{s_{p}\left(x_{p}\right)} \rightarrow \mathcal{H}^{0}, \quad \eta \mapsto x_{p} \cdot \eta .
$$


We define the range and source maps $\tilde{r}_{p}, \tilde{s}_{p}: \tilde{\mathcal{X}}_{p} \rightarrow \mathcal{H}^{0}$ as

$$
\tilde{r}_{p}\left(x_{p}, \eta\right):=x_{p} \cdot \eta, \quad \tilde{s}_{p}\left(x_{p}, \eta\right):=\eta
$$

for all $\left(x_{p}, \eta\right) \in \mathcal{X}_{p} \times_{s_{p}, \mathcal{G}^{0}, r} \mathcal{H}^{0}$.

The right action of $\tilde{\mathcal{G}}$ on $\tilde{\mathcal{X}}$ is given by

$$
\left(x_{p}, \eta\right) \cdot(g, \zeta):=\left(x_{p} g, g^{-1} \eta\right)
$$

for all $\left(\left(x_{p}, \eta\right),(g, \zeta)\right) \in \tilde{\mathcal{X}}_{p} \times_{\tilde{s}, \mathcal{H}^{0}, r_{\tilde{\mathcal{G}}}} \tilde{\mathcal{G}}$. And the left action is given by

$$
(g, \zeta) \cdot\left(x_{p}, \eta\right)=\left(g \cdot x_{p}, \eta\right)
$$

for all $\left((g, \zeta),\left(x_{p}, \eta\right)\right) \in \tilde{\mathcal{G}} \times_{s_{\tilde{\mathcal{G}}}, \mathcal{H}^{0}, \tilde{r}_{p}} \tilde{\mathcal{X}}_{p}$

Lemma 3.26. The map $\tilde{s}_{p}: \tilde{\mathcal{X}}_{p} \rightarrow \mathcal{H}^{0}$ is a local homeomorphism for all $p \in P$. It is surjective if $s_{p}$ is surjective.

Proof. We may use the following pullback diagram

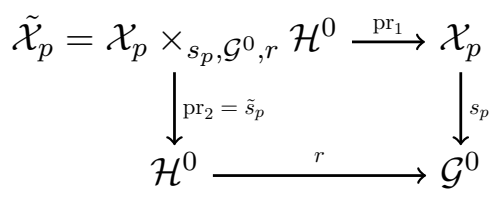

The source map $s_{p}: \mathcal{X}_{p} \rightarrow \mathcal{G}^{0}$ is a local homeomorphism, hence so is the parallel map $\tilde{s}$. Similarly, $\tilde{s}_{p}$ is surjective if $s_{p}$ is.

Lemma 3.27. The map $\tilde{r}_{p}$ induces a homeomorphism $\tilde{r}_{p_{*}}: \tilde{\mathcal{X}}_{p} / \mathcal{G} \rightarrow \mathcal{H}^{0}$ for all $p \in P$.

Proof. To see that the map $\tilde{r}_{p_{*}}$ is surjective, let $\eta=\left(\eta_{p}\right)_{p \in P} \in \mathcal{H}^{0}$. Let $\eta_{p}^{\prime} \in \mathcal{X}_{p}$ be such that $\mathrm{p}_{p}\left(\eta_{p}^{\prime}\right)=\eta_{p}$. For all $t \in P$ we write $\zeta_{t}:=S_{\eta_{p}^{\prime}, \mathcal{X}_{t}}\left(\eta_{p t}\right)$. Recall from Equation (3.26) that $S_{\eta_{p}^{\prime}, \mathcal{X}_{t}}\left(\eta_{p t}\right)=\mathrm{pr}_{2} \circ \mathrm{p}^{-1}\left(\eta_{p t}\right)$, where p: $C_{\eta_{p}^{\prime}} \subset \mathcal{X}_{p} \times_{s_{p}, \mathcal{G}^{0}, r_{t}} \mathcal{X}_{t} \rightarrow$ $\mathcal{X}_{p} \circ \mathcal{G} \mathcal{X}_{t}$ is the quotient map. Let $q \in P$. Then $r_{q, t}\left(\zeta_{q t}\right)=r_{q, t} \circ S_{\eta_{p}^{\prime}, \mathcal{X}_{q t}}\left(\eta_{p q t}\right)$. But $r_{q, t} \circ S_{\eta_{p}^{\prime}, \mathcal{X}_{q t}}=S_{\eta_{p}^{\prime}, \mathcal{X}_{q}} \circ r_{p q, t}$. This implies that

$$
r_{q, t}\left(\zeta_{q t}\right)=S_{\eta_{p}^{\prime}, \mathcal{X}_{q}} \circ r_{p q, t}\left(\eta_{p q t}\right)=S_{\eta_{p}^{\prime}, \mathcal{X}_{q}}\left(\eta_{p q}\right)=\zeta_{q}
$$

and therefore $\zeta \in \mathcal{H}^{0}$. By construction, $\left(\eta_{p}^{\prime} \cdot \zeta\right)_{p t}=\eta_{p t}$ for all $t \in P$. Let $b \in P$. Since $P$ is an Ore monoid we may find $k_{1}$ and $k_{2} \in P$ with $b k_{1}=p k_{2}$. We define

$$
\left(\eta_{p}^{\prime} \cdot \zeta\right)_{b}=r_{b, k_{1}}\left(\left(\eta_{p}^{\prime} \cdot \zeta\right)_{p k_{2}}\right)=r_{b, k_{1}}\left(\eta_{p k_{2}}\right)=r_{b, k_{1}}\left(\eta_{b k_{1}}\right)=\eta_{b}
$$




\section{Actions of Ore monoids}

Thus $\eta_{p}^{\prime} \cdot \zeta=\eta$ and $\tilde{r}_{p_{*}}$ is surjective. To see that $\tilde{r}_{p_{*}}$ is injective, let $\left(x_{p}, \xi\right),\left(y_{p}, \eta\right) \in$ $\tilde{\mathcal{X}}_{p}$ with $x_{p} \cdot \xi=y_{p} \cdot \eta$. So $\mathrm{p}_{p t} \circ \sigma_{p, t}^{-1}\left(y_{p}, \eta_{t}^{\prime}\right)=\mathrm{p}_{p t} \circ \sigma_{p, t}^{-1}\left(x_{p}, \xi_{t}^{\prime}\right)$ for all $t \in P$. Hence $\sigma_{p, t}^{-1}\left(y_{p}, \eta_{t}^{\prime}\right)=\sigma_{p, t}^{-1}\left(x_{p}, \xi_{t}^{\prime}\right) h$ for an element $h \in \mathcal{G}$. This means that there is an element $g \in \mathcal{G}$ such that $r(g)=s_{p}\left(x_{p}\right)$ and

$$
\left(y_{p} g, g^{-1} \eta_{t}^{\prime}\right)=\left(x_{p}, \xi_{t}^{\prime} \cdot h\right) .
$$

Consequently, the points $\left(x_{p}, \xi\right)$ and $\left(y_{p}, \eta\right)$ represent the same point in $\tilde{\mathcal{X}}_{p} / \mathcal{G}$. Finally, the map $\tilde{r}_{p_{*}}$ is open and continuous since the space $\mathcal{H}^{0}$ is endowed with the projective limit topology and $\tilde{r}_{p_{*}}$ is given by the maps $\sigma_{t}^{-1}$ and $\mathrm{p}_{t}$, see Equation (3.32), which are both open and continuous for all $t \in P$.

Lemma 3.28. $\quad \tilde{\mathcal{X}}_{p}$ is a tight correspondence over the groupoid $\tilde{\mathcal{G}}$ for all $p \in P$. The left action of $\tilde{\mathcal{G}}$ on $\tilde{\mathcal{X}}_{p}$ is free if the left action of $G$ on $\mathcal{X}_{p}$ is free.

Proof. The defining formulas of the actions (3.37), (3.36) show that the right and left actions of the groupoid $\tilde{\mathcal{G}}$ on $\tilde{\mathcal{X}}_{p}$ commute; the right action of $\tilde{\mathcal{G}}$ on $\tilde{\mathcal{X}}_{p}$ is free and proper since the right action of $\mathcal{G}$ on $\mathcal{X}_{p}$ is free and proper and the left action of $\tilde{\mathcal{G}}$ on $\tilde{\mathcal{X}}_{p}$ is free if the left action of $G$ on $\mathcal{X}_{p}$ is free. The source map on $\tilde{\mathcal{X}}$ is a local homeomorphism By Lemma 3.26. The map $\tilde{r}_{p_{*}}: \tilde{\mathcal{X}}_{p} / \mathcal{G} \rightarrow \mathcal{H}^{0}$ is a homeomorphism by Lemma 3.27.

Next we show that the isomorphisms $\sigma_{p, q}: \mathcal{X}_{p q} \rightarrow \mathcal{X}_{p} \circ_{\mathcal{G}} \mathcal{X}_{q}$ lift to isomorphisms $\tilde{\sigma}_{p, q}: \tilde{\mathcal{X}}_{p q} \rightarrow \tilde{\mathcal{X}}_{p} \circ_{\tilde{\mathcal{G}}} \tilde{\mathcal{X}}_{q}$. Let $\tau_{p, q}: \tilde{\mathcal{X}}_{p} \circ \tilde{\mathcal{G}}_{\tilde{\mathcal{X}}} \rightarrow \tilde{\mathcal{X}}_{p q}$ be the map given by

$$
\tau_{p, q}\left(\left[x_{p}, \xi, y_{q}, \eta\right]\right):=\left(\sigma^{-1}\left[x_{p}, y_{q}\right], \eta\right)
$$

for all $\left[x_{p}, \xi, y_{q}, \eta\right] \in \tilde{\mathcal{X}}_{p} \circ_{\tilde{\mathcal{G}}} \tilde{\mathcal{X}}_{q}$.

Lemma 3.29. $\tau_{p, q}: \tilde{\mathcal{X}}_{p} \circ_{\tilde{\mathcal{G}}} \tilde{\mathcal{X}}_{q} \rightarrow \tilde{\mathcal{X}}_{p q}$ is an isomorphism for all $p, q \in P$.

Proof. First we have to check that Equation (3.38) gives a well-defined map. If $\left[x_{p}, \xi, y_{q}, \eta\right]=\left[x_{p}^{1}, \xi^{1}, y_{q}^{1}, \eta^{1}\right] \in \tilde{\mathcal{X}}_{p} \circ_{\tilde{\mathcal{G}}} \tilde{\mathcal{X}}_{q}$, then there is an element $(g, \zeta) \in \tilde{\mathcal{G}}$ such that $g \zeta=\xi$ and $\left(x_{p} g, g^{-1} \xi, g^{-1} y_{p}, \eta\right)=\left(x_{p}^{1}, \xi^{1}, y_{q}^{1}, \eta^{1}\right)$ as elements in the space $\mathcal{X}_{p} \times_{s_{p}, \mathcal{G}^{0}, r} \mathcal{H}^{0} \times_{\tilde{s}_{p}, \mathcal{H}^{0}, \tilde{r}_{q}} \mathcal{X}_{q} \times_{s_{q}, \mathcal{G}^{0}, r} \mathcal{H}^{0}$. Thus $\eta^{1}=\eta, x_{p} g=x_{p}^{1}, g^{-1} y_{q}=y_{q}^{1}$. Then

$$
\begin{aligned}
\tau_{p, q}\left(\left[x_{p}^{1}, \xi^{1}, y_{q}^{1}, \eta^{1}\right]\right) & =\left(\sigma_{p, q}^{-1}\left[x_{p} g, g^{-1} y_{q}\right], \eta\right) \\
& =\left(\sigma_{p, q}^{-1}\left[x_{p}, y_{q}\right], \eta\right) \\
& =\tau_{p, q}\left(\left[x_{p}, \xi, y_{q}, \eta\right]\right)
\end{aligned}
$$

so the map $\tau_{p, q}$ is well defined. Now let $\left[x_{p}, \xi, y_{q}, \eta\right],\left[x_{p}^{1}, \xi^{1}, y_{q}^{1}, \eta^{1}\right] \in \tilde{\mathcal{X}}_{p}{ }_{\tilde{\mathcal{G}}} \tilde{\mathcal{X}}_{q}$ be such that $\tau_{p, q}\left(\left[x_{p}, \xi, y_{q}, \eta\right]\right)=\tau_{p, q}\left(\left[x_{p}^{1}, \xi^{1}, y_{q}^{1}, \eta^{1}\right]\right)$. Then

$$
\left(\sigma_{p, q}^{-1}\left[x_{p}, y_{q}\right], \eta\right)=\left(\sigma_{p, q}^{-1}\left[x_{p}^{1}, y_{q}^{1}\right], \eta^{1}\right) .
$$


Hence $\eta=\eta^{1}$ and $\sigma_{p, q}^{-1}\left[x_{p}, y_{q}\right]=\sigma_{p, q}^{-1}\left[x_{p}^{1}, y_{q}^{1}\right]$. Since $\sigma_{p, q}$ is an isomorphism, there is an element $g \in \mathcal{G}$ such that $\left(x_{p} g, g^{-1} y_{q}\right)=\left(x_{p}^{1}, y_{q}^{1}\right)$ in $\mathcal{X}_{p} \times_{s_{p}, \mathcal{G}^{0}, r_{q}} \mathcal{X}_{q}$. Thus $x_{p} g=$ $x_{p}^{1}, g^{-1} y_{q}=y_{q}^{1}$. In addition, $\xi^{1}=y_{q}^{1} \cdot \eta=g^{-1} y_{q} \cdot \eta=g^{-1} \xi$. So

$$
\left[x_{p}^{1}, \xi^{1}, y_{q}^{1}, \eta\right]=\left[x_{p} g, g^{-1} \xi, g^{-1} y_{q}, \eta\right]=\left[x_{p}, \xi, y_{q}, \eta\right] .
$$

Then $\tau_{p, q}$ is injective. Next let $\left(x_{p q}, \eta\right) \in \tilde{\mathcal{X}}_{p q}$. Since $\sigma_{p, q}$ is an isomorphism, there is $\left(x_{p}^{1}, x_{q}^{2}\right) \in \mathcal{X}_{p} \times_{s_{p}, \mathcal{G}^{0}, r_{q}} \mathcal{X}_{q}$ with $\sigma\left(x_{p q}\right)=\left[x_{p}^{1}, x_{q}^{2}\right]$. Then $\left(x_{p}^{1}, x_{q}^{2} \cdot \eta\right) \in \tilde{\mathcal{X}}_{p},\left(x_{q}^{2}, \eta\right) \in$ $\tilde{\mathcal{X}}_{q},\left(x_{p}^{1}, x_{q}^{2} \cdot \eta, x_{q}^{2}, \eta\right) \in \mathcal{X}_{p} \times_{s_{p}, \mathcal{G}^{0}, r} \mathcal{H}^{0} \times_{\tilde{s}_{p}, \mathcal{H}^{0}, \tilde{r}_{q}} \mathcal{X}_{q} \times_{s_{q}, \mathcal{G}^{0}, r} \mathcal{H}^{0}$ and $\tau\left(x_{p}^{1}, x_{q}^{2} \cdot \eta, x_{q}^{2}, \eta\right)=$ $\left(\sigma_{p, q}^{-1}\left[x_{p}^{1}, x_{q}^{2}\right], \eta\right)=\left(x_{p q}, \eta\right)$. Then $\tau_{p, q}$ is surjective. It is also open and continuous since $\sigma_{p, q}^{-1}$ is a homeomorphism. To see that $\tau_{p, q}$ is $\tilde{\mathcal{G}}$-equivariant, let $\left[x_{p}, \xi, y_{q}, \eta\right] \in$ $\tilde{\mathcal{X}} \circ_{\tilde{\mathcal{G}}} \tilde{\mathcal{X}}_{q}$ and let $(g, \zeta) \in \tilde{\mathcal{G}}$ satisfy $g \zeta=\eta$. We have

$$
\begin{aligned}
\tau_{p, q}\left(\left[x_{p}, \xi, y_{q}, \eta\right]\right) \cdot(g, \zeta) & =\left(\sigma_{p, q}^{-1}\left[x_{p}, y_{q}\right], \eta\right) \cdot g \\
& =\left(\sigma_{p, q}^{-1}\left[x_{p}, y_{q}\right] g, g^{-1} \eta\right)=\left(\sigma_{p, q}^{-1}\left[x_{p}, y_{q} g\right], g^{-1} \eta\right) \\
& =\tau_{p, q}\left(\left[x_{p}, \xi, y_{q} g, g^{-1} \eta\right]\right)=\tau_{p, q}\left(\left[x_{p}, \xi, y_{q}, \eta\right] \cdot(g, \zeta)\right) .
\end{aligned}
$$

Similarly, $(g, \zeta) \cdot \tau_{p, q}\left(\left[x_{p}, \xi, y_{q}, \eta\right]\right)=\tau_{p, q}\left((g, \zeta)\left[x_{p}, \xi, y_{q}, \eta\right]\right)$ for all $\left[x_{p}, \xi, y_{q}, \eta\right] \in$ $\tilde{\mathcal{X}}_{\tilde{\mathcal{G}}_{\mathcal{G}}} \mathcal{X}_{q}$ and all $(g, \zeta) \in \tilde{\mathcal{G}}$ with $\zeta=\sigma_{p, q}^{-1}\left[x_{p}, y_{q}\right] \cdot \eta$. Since $\xi=y_{q} \cdot \eta$, we have $\tilde{r}_{p}\left(x_{p}, \xi\right)=$ $x_{p} \cdot \xi=\sigma_{p, q}^{-1}\left[x_{p}, y_{q}\right] \cdot \eta=\zeta$.

For all $p, q \in P$, we set $\tilde{\sigma}_{p, q}:=\tau_{p, q}^{-1}$. The maps $\tilde{\sigma}_{p, q}$ satisfy Equation (3.1), since the maps $\sigma_{p, q}$ satisfy the same condition, being part of an action of $P$ on $\mathcal{G}$ by proper correspondences. Moreover, the correspondence $\tilde{\mathcal{X}}_{p}$ is tight for all $p \in P$ by Lemma 3.28. It follows that $\left(\tilde{\mathcal{X}}_{p}, \tilde{\sigma}_{p, q}\right)$ defines an action of $P$ on $\tilde{\mathcal{G}}$ by tight correspondences. Hence it has a colimit by Theorem 3.15. It remains to show that the colimit of this action is a colimit of the original diagram.

Theorem 3.30. The diagrams $\left(\mathcal{X}_{p}, \sigma_{p, q}\right)$ and $\left(\tilde{\mathcal{X}}_{p}, \tilde{\sigma}_{p, q}\right)$ have the same colimit in $\mathfrak{G r}$.

Proof. The colimit of the diagram $\left(\tilde{\mathcal{X}}_{p}, \tilde{\sigma}_{p, q}\right)$ exists by Theorem 3.15. We set

$$
\mathcal{H}:=\operatorname{colim}\left(\tilde{\mathcal{X}}_{p}, \tilde{\sigma}_{p, q}\right) \text {. }
$$

We will show that the groupoid $\mathcal{H}$ given by Equation (3.40) is also a colimit of the diagram $\left(\mathcal{X}_{p}, \sigma_{p, q}\right)$. This is sufficient since the colimit of a diagram is unique (up to isomorphism) if it exists.

Let $\mathcal{D}$ be a groupoid and let $\left(\mathcal{Y}, \varphi_{p}\right)$ be a transformation from $\left(\mathcal{X}_{p}, \sigma_{p, q}\right)$ to const $_{\mathcal{D}}$. For all $p \in P$, the isomorphism $\varphi_{p}^{-1}: \mathcal{Y} \rightarrow \mathcal{X}_{p} \circ_{\mathcal{G}} \mathcal{Y}$, composed with the projection $r_{\mathcal{X}_{p}, \mathcal{Y}}: \mathcal{X}_{p} \circ_{\mathcal{G}} \mathcal{Y} \rightarrow \mathcal{X}_{p} / \mathcal{G}$, gives a map $\psi_{p}: \mathcal{Y} \rightarrow \mathcal{X}_{p} / \mathcal{G}$. On the one hand, we have

$$
r_{p, q} r_{\mathcal{X}_{p q}, \mathcal{Y}}\left(\sigma_{p, q}^{-1} \times_{\mathcal{G}} \operatorname{id} \mathcal{Y}\right)\left(\operatorname{id}_{\mathcal{X}_{p}} \times_{\mathcal{G}} \varphi_{q}^{-1}\right)=r_{\mathcal{X}_{p}, \mathcal{Y}}
$$




\section{Actions of Ore monoids}

for all $p, q \in P$. So

$$
r_{p, q} r_{\mathcal{X}_{p q}, \mathcal{Y}}\left(\sigma_{p, q}^{-1} \times_{\mathcal{G}} \operatorname{id} \mathcal{Y}\right)\left(\operatorname{id}_{\mathcal{X}_{p}} \times_{\mathcal{G}} \varphi_{q}^{-1}\right) \varphi_{p}^{-1}=r_{\mathcal{X}_{p}, \mathcal{Y}} \varphi_{p}^{-1}
$$

for all $p, q \in P$. On the other hand, the maps $\varphi_{p}$ satisfy the coherence condition (2.27). Therefore,

$$
\left(\sigma_{p, q}^{-1} \times_{\mathcal{G}} \operatorname{id} \mathcal{Y}\right)\left(\operatorname{id}_{\mathcal{X}_{p}} \times_{\mathcal{G}} \varphi_{q}^{-1}\right) \varphi_{p}^{-1}=\varphi_{p q}^{-1} .
$$

Thus

$$
r_{p, q} \psi_{p q}=\psi_{p}
$$

Equation (3.41) says that $\left(\psi_{p}\right)_{p \in P}$ is an inverse limit map from $\mathcal{Y}$ to $\left(\mathcal{X}_{p} / \mathcal{G}, r_{p, q}\right)$. Hence it induces a continuous map $\psi_{\infty}=\lim _{\longleftarrow} \psi_{p}: \mathcal{Y} \rightarrow \mathcal{H}^{0}=\lim _{\longleftarrow} \mathcal{X}_{p} / \mathcal{G}$. We define a left action of $\tilde{\mathcal{G}}$ on $\mathcal{Y}$, using $\psi_{\infty}$ as a left anchor map, by setting

$$
(g, \xi) \cdot y:=g \cdot y,
$$

for all $(g, \xi) \in \tilde{\mathcal{G}}, y \in \mathcal{Y}$ with $\psi_{\infty}(y)=\xi$. This action is well defined and commutes with the right action of $\mathcal{D}$. This implies that $\mathcal{Y}$ is also a correspondence from $\tilde{\mathcal{G}}$ to $\mathcal{D}$.

Let $p \in P$. We want to extend the isomorphism $\varphi_{p}: \mathcal{X}_{p} \circ_{\mathcal{G}} \mathcal{Y} \rightarrow \mathcal{Y}$ to an isomorphism $\tilde{\varphi}_{p}: \tilde{\mathcal{X}}_{p} \circ \tilde{\mathcal{G}} \mathcal{Y} \rightarrow \mathcal{Y}$, so that $\left(\tilde{\mathcal{X}}_{p}, \tilde{\varphi}_{p}\right)$ becomes a natural transformation from $\left(\tilde{\mathcal{X}}_{p}, \tilde{\sigma}_{p, q}\right)$ to const $_{\mathcal{D}}$. We claim that the map

$$
\tilde{\varphi}_{p}: \tilde{\mathcal{X}}_{p} \circ_{\tilde{\mathcal{G}}} \mathcal{Y} \rightarrow \mathcal{Y}, \quad\left(\left(x_{p}, \xi\right), y\right) \mapsto \varphi_{p}\left(x_{p}, y\right),
$$

achieves this.

Lemma 3.31. For all $p \in P, \tilde{\varphi}_{p}: \tilde{\mathcal{X}}_{p} \circ_{\tilde{\mathcal{G}}} \mathcal{Y} \rightarrow \mathcal{Y}$ is an isomorphism of correspondences. $\left(\mathcal{Y}, \tilde{\varphi}_{p}\right)$ is a transformation from $\left(\tilde{\mathcal{X}}_{p}, \tilde{\sigma}_{p, q}\right)$ to the constant diagram const $_{\mathcal{D}}$.

Proof. First, let $\left(\left(x_{p}, \xi\right), y\right) \in \tilde{\mathcal{X}}_{p} \times_{s_{\tilde{\mathcal{G}}} \tilde{\mathcal{G}}^{0}, \psi_{\infty}} \mathcal{Y}$ and let $(g, \eta) \in \tilde{\mathcal{G}}$ with $\psi_{\infty}(y)=$ $g \eta=r_{\tilde{\mathcal{G}}}(g, \eta)$. Then

$$
\begin{aligned}
\tilde{\varphi}_{p}\left(\left(x_{p}, \xi\right)(g, \eta),(g, \eta)^{-1} y\right) & =\tilde{\varphi}_{p}\left(\left(x_{p} g, g^{-1} \xi\right), g^{-1} y\right) \\
& =\varphi_{p}\left(x_{p} g, g^{-1} y\right)=\varphi_{p}\left(x_{p}, y\right) \\
& =\tilde{\varphi}_{p}\left(\left(x_{p}, \xi\right), y\right)
\end{aligned}
$$

It follows that $\tilde{\varphi}_{p}$ is a well defined map. Now let $\left(\left(x_{p}, \xi\right), y\right) \in \tilde{\mathcal{X}}_{p} \times_{s_{\tilde{\mathcal{G}}}, \tilde{\mathcal{G}}^{0}, \psi_{\infty}}$ $\mathcal{Y}$ and let $\left(\left(x_{p}^{\prime}, \xi^{\prime}\right), y^{\prime}\right) \in \tilde{\mathcal{X}}_{p} \times_{s_{\tilde{\mathcal{G}}}, \tilde{\mathcal{G}}^{0}, \psi_{\infty}} \mathcal{Y}$ with $\tilde{\varphi}_{p}\left(\left(x_{p}, \xi\right), y\right)=\tilde{\varphi}_{p}\left(\left(x_{p}^{\prime}, \xi^{\prime}\right), y^{\prime}\right)$. 
Then $\varphi_{p}\left(x_{p}, y\right)=\varphi_{p}\left(x_{p}^{\prime}, y^{\prime}\right)$. Since $\varphi_{p}$ is an isomorphism, there is an element $g \in \mathcal{G}$ such that $r_{\mathcal{G}}(g)=r_{\mathcal{Y}}(y)$ and $\left(x_{p}^{\prime}, y^{\prime}\right)=\left(x_{p} g, g^{-1} y\right)$. This implies $x_{p}^{\prime}=x_{p} g, y^{\prime}=$ $g^{-1} y$ and hence $\xi^{\prime}=\psi_{\infty}\left(g^{-1} y\right)=g^{-1} \psi_{\infty}(y)=g^{-1} \xi$. The element $\left(g, g^{-1} \xi\right) \in \tilde{\mathcal{G}}$ satisfies

$$
\left(\left(x_{p}, \xi\right)\left(g, g^{-1} \xi\right),\left(g, g^{-1} \xi\right)^{-1} y\right)=\left(\left(x_{p} g, g^{-1} \xi\right), g^{-1} y\right)=\left(\left(x_{p}^{\prime}, \xi^{\prime}\right), y^{\prime}\right) .
$$

Then $\left[\left(x_{p}^{\prime}, \xi^{\prime}\right), y^{\prime}\right]=\left[\left(x_{p}, \xi\right), y\right] \in \tilde{\mathcal{X}}_{p} \circ_{\tilde{\mathcal{G}}} \mathcal{Y}$. It follows that the map $\tilde{\varphi}_{p}$ is injective. Next let $y \in \mathcal{Y}$ and let $\left(x_{p}, z\right) \in \mathcal{X}_{p} \times_{s_{p}, \mathcal{G}^{0}, r_{\mathcal{Y}}} \mathcal{Y}$ be such that $\varphi_{p}\left(x_{p}, z\right)=y$. Then the point $x=\left(\left(x_{p}, \varphi_{\infty}(z)\right), z\right)$ satisfies $\tilde{\varphi}_{p}([x])=y$. Thus the map $\tilde{\varphi}_{p}$ is surjective. The definition of the left action of $\tilde{\mathcal{G}}$ on $\tilde{\mathcal{X}}_{p}$ and the fact that $\varphi_{p}$ is $\mathcal{G}, \mathcal{D}$-equivariant imply that $\tilde{\varphi}_{p}$ is a $\tilde{\mathcal{G}}, \mathcal{D}$-equivariant.

Finally, the map $\left(\varphi_{p}\right)_{p \in P}$ satisfies condition (2.29). Therefore, the definition of the isomorphisms $\tilde{\sigma}_{p, q}$ implies that $\left(\tilde{\varphi}_{p}\right)_{p \in P}$ satisfies the same condition. We have the pullback diagram

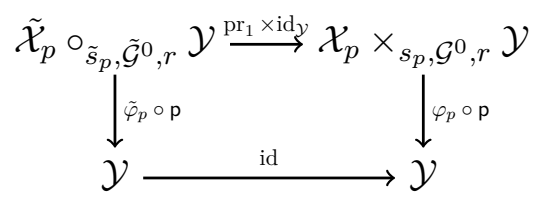

So $\tilde{\varphi}_{p} \circ \mathrm{p}$ is open and continuous since $\varphi_{p} \circ \mathrm{p}$ is. Hence $\tilde{\varphi}_{p}$ is open and continuous since $p$ is open and continuous. This concludes the proof of the lemma.

Conversely, let $\left(\mathcal{Y}, \tilde{\varphi}_{p}\right)$ be a transformation from $\left(\tilde{\mathcal{X}}_{p}, \tilde{\sigma}_{p, q}\right)$ to const $_{\mathcal{D}}$. There is a natural action of $\mathcal{G}$ on $\mathcal{Y}$. The right anchor map is given by $r_{\mathcal{Y}}^{\mathcal{Y}}(y)=r_{\mathcal{G}}\left(r_{\mathcal{Y}}(y)\right)$. The action is defined by

$$
g \cdot y=\left(g, r_{\mathcal{Y}}(y)\right) \cdot y
$$

for all $y \in \mathcal{Y}$ and $g \in \mathcal{G}$ with $r_{\mathcal{Y}}^{\mathcal{Y}}(y)=s(g)$.

This action commutes with the right action of $\mathcal{D}$ on $\mathcal{Y}$. Next we define

$$
\varphi_{p}: \tilde{\mathcal{X}}_{p} \times_{s_{p}, \mathcal{G}^{0}, r_{\mathcal{Y}}} \mathcal{Y} \rightarrow \mathcal{Y}, \quad\left(x_{p}, y\right) \mapsto \tilde{\varphi}_{p}\left(\left(x_{p}, r_{\mathcal{Y}}(y)\right), y\right)
$$

Using similar arguments as in Lemma 3.31, we see that $\left(\varphi_{p}, \mathcal{Y}\right)$ is a transformation from $\left(\mathcal{X}_{p}, \sigma_{p, q}\right)$ to the constant diagram const $_{\mathcal{D}}$. The two constructions $\left(\mathcal{Y}, \varphi_{p}\right) \mapsto\left(\mathcal{Y}, \tilde{\varphi}_{p}\right)$ and $\left(\mathcal{Y}, \tilde{\varphi}_{p}\right) \mapsto\left(\mathcal{Y}, \varphi_{p}\right)$ are inverse to each other. Moreover, a modification between two transformations $\left(\varphi_{p}, \mathcal{Y}\right)$ and $\left(\varphi_{p}^{\prime}, \mathcal{Y}^{\prime}\right)$ is an isomorphism $w: \mathcal{Y} \rightarrow \mathcal{Y}^{\prime}$ of $\mathcal{G}, \mathcal{D}$-correspondences that intertwines $\varphi_{p}$ and $\varphi_{p}^{\prime}$, see Equation (2.30). Equation (3.42) says that $w$ is an isomorphism of $\tilde{\mathcal{G}}, \mathcal{D}$-correspondences 


\section{Actions of Ore monoids}

and Equation (3.43) says that it intertwines $\tilde{\varphi}_{p}$ and $\tilde{\varphi}_{p}^{\prime}$. Consequently, $w$ is a modification from $\left(\mathcal{Y}, \tilde{\varphi}_{p}\right)$ to $\left(\mathcal{Y}^{\prime}, \tilde{\varphi}_{p}^{\prime}\right)$. Similarly, by Equation (3.44) and Equation (3.45), a modification between two transformations $\left(\mathcal{Y}, \tilde{\varphi}_{p}\right)$ to $\left(\mathcal{Y}^{\prime}, \tilde{\varphi}_{p}^{\prime}\right)$ is the same as a modification between $\left(\mathcal{Y}, \varphi_{p}\right)$ and $\left(\mathcal{Y}^{\prime}, \varphi_{p}^{\prime}\right)$. Thus we have an equivalence between the groupoids $\mathfrak{C o r r}^{P}\left(\left(\mathcal{X}_{p}, \sigma_{p, q}\right)\right.$, const $\left._{\mathcal{D}}\right)$ and $\operatorname{Corr}^{P}\left(\left(\tilde{\mathcal{X}}_{p}, \tilde{\sigma}_{p, q}\right)\right.$, const $\left.{ }_{\mathcal{D}}\right)$. Therefore, the diagrams $\left(\tilde{\mathcal{X}}_{p}, \tilde{\sigma}_{p, q}\right)$ and $\left(\mathcal{X}_{p}, \sigma_{p, q}\right)$ have the same colimit in $\mathfrak{G r}$. This colimit is $\mathcal{H}$ by Theorem 3.15 .

\subsubsection{The product systems}

Let $\mathcal{L}$ be an étale groupoid. Let $S \subset \operatorname{Bis}(\mathcal{L})$ be an inverse semigroup. Let $u \in S$ be a bisection (see Definition 3.5). Then the map $\alpha(u): r(u) \rightarrow s(u), s(g) \mapsto r(g)$ is a homeomorphism. And the map

$$
\alpha: S \mapsto I\left(\mathcal{L}^{0}\right), \quad u \mapsto \alpha(u),
$$

is a semigroup homomorphism. See [16, Proposition 5.3]. This gives an action of $S$ on $\mathcal{L}^{0}$. Furthermore, the action of $S$ on $\mathcal{L}^{0}$ by partial homeomorphisms induces an action of $S$ on $\mathrm{C}_{0}\left(\mathcal{L}^{0}\right)$ by partial isomorphisms, that is, an action by isomorphisms between closed ideals in $\mathrm{C}_{0}\left(\mathcal{L}^{0}\right)$. For simplicity, we also denote the induced action by $\alpha$. In what follows, we assume that $S$ is wide inverse semigroup. That is, the inclusion map $S \rightarrow \operatorname{Bis}(\mathcal{L})$ is a wide representation, see [28, Definition 2.18], and $\bigcup_{u \in S} u=\mathcal{L}^{1}$. Equivalently, the transformation groupoid $S \ltimes \mathcal{L}^{0}$ is isomorphic to the groupoid $\mathcal{L}$. For instance, we may take $S=\operatorname{Bis}(\mathcal{L})$ (see $[9$, Proposition 5.1]). For a wide inverse semigroup $S \subset \operatorname{Bis}(\mathcal{G})$, the groupoid $\mathrm{C}^{*}$-algebra $\mathrm{C}^{*}(\mathcal{L})$ is naturally isomorphic to the crossed product $S \ltimes \mathrm{C}_{0}\left(\mathcal{L}^{0}\right)$ (see [16, Proposition 9.7]).

Definition 3.32. Let $S$ be a unital inverse semigroup. Let $D$ be a $\mathrm{C}^{*}$-algebra and $\mathcal{F}$ a Hilbert $D$-module. A representation of $S$ on $\mathcal{F}$ is a semigroup homomorphism from $S$ to the inverse semigroup $I(\mathcal{F})$ of isomorphisms between sub-Hilbert modules of $\mathcal{F}$. Let $\alpha: S \rightarrow I(\mathcal{F})$ be a representation. For $u \in S$, let $\mathcal{F}_{u}$ be the domain of the isomorphism $\alpha(u)$.

Definition 3.33. Let $X$ be a locally compact Hausdorff space. And let $\alpha: S \rightarrow$ $I(X)$ be an action of $S$ on $X$ by partial homeomorphisms. Let $E_{u}$ denote the domain of the homeomorphism $\alpha(u)$. We also denote by $\alpha$ the action of $S$ by partial isomorphisms on $\mathrm{C}_{0}(X)$. A covariant representation of $(S, X)$ is a pair $(\beta, \mathcal{F})$, where $\mathcal{F}$ is a correspondence from $\mathrm{C}_{0}(X)$ to a $\mathrm{C}^{*}$-algebra $D$ and $\beta$ is a representation of $S$ on $\mathcal{F}$ such that

(1) the domain of $\beta(u)$ is $\mathcal{F}_{u}=\mathrm{C}_{0}\left(E_{u^{*}}\right) \mathcal{F}$ and its codomain is $\mathrm{C}_{0}\left(E_{u}\right) \mathcal{F}$; 
3.3 Actions of Ore monoids by proper correspondences

(2) $\beta(u) \varphi_{\mathrm{C}_{0}(X)}(a) \beta(u)^{*}=\varphi_{\mathrm{C}_{0}(X)}(\alpha(u)(a))$

for all $u \in S$ and all $a \in \mathrm{C}_{0}\left(E_{u^{*}}\right)$.

Covariant representations of inverse semigroup actions have been introduced in [43, Definition 4.5], see also [16, Definition 8.1].

Proposition 3.34. Let $\mathcal{L}$ be an étale groupoid and let $S \subset \operatorname{Bis}(\mathcal{L})$ be a wide inverse semigroup. Let $D$ be a $\mathrm{C}^{*}$-algebra. Then there is a bijection between the set of correspondences $\mathcal{F}: \mathrm{C}^{*}(\mathcal{L}) \rightarrow D$ and the set of covariant representations of $\left(S, \mathcal{L}^{0}\right)$ on Hilbert D-modules.

Proof. Let $\mathcal{F}: \mathrm{C}^{*}(\mathcal{L}) \rightarrow D$ be a correspondence. The correspondence $\mathcal{L}: \mathcal{L}^{0} \rightarrow \mathcal{L}$ is proper. Hence, by Proposition 2.38, the $\mathrm{C}^{*}$-correspondence $\mathrm{C}^{*}(\mathcal{L}): \mathrm{C}_{0}\left(\mathcal{L}^{0}\right) \rightarrow$ $\mathrm{C}^{*}(\mathcal{L})$ is a proper.

We denote $B_{u}:=\mathrm{C}_{0}\left(u^{*}\right) \mathcal{F}$ and

$$
\beta(u): B_{u^{*}} \rightarrow B_{u}, \quad f \cdot \xi \mapsto \alpha(u)(f) \cdot \xi .
$$

See (3.46) for the definition of $\alpha$. Then we have

$$
\begin{aligned}
\left\langle\beta(u)\left(f_{1} \cdot \xi_{1}\right), \beta(u)\left(f_{2} \cdot \xi_{2}\right)\right\rangle & =\left\langle\alpha(u)\left(f_{1}\right) \cdot \xi_{1}, \alpha(u)\left(f_{2}\right) \cdot \xi_{2}\right\rangle \\
& =\left\langle\xi_{1},\left(\alpha(u)\left(f_{1}\right)\right)^{*} \alpha(u)\left(f_{2}\right) \cdot \xi_{2}\right\rangle \\
& =\left\langle\xi_{1}, \alpha\left(u^{*} u\right)\left(f_{1}^{*} f_{2}\right) \cdot \xi_{2}\right\rangle \\
& =\left\langle\xi_{1}, f_{1}^{*} f_{2} \cdot \xi_{2}\right\rangle=\left\langle f_{1} \cdot \xi_{1}, f_{2} \cdot \xi_{2}\right\rangle .
\end{aligned}
$$

It follows that $\beta(u)$ is an isometry. It is surjective since $\alpha(u)$ is an isomorphism. Hence $\beta(u): B_{u} \rightarrow B_{u^{*}}$ is an isomorphism of Hilbert $D$-modules. We will show that $(\beta, \mathcal{F})$ is a covariant representation. Condition $(1)$ is satisfied by construction. Next we check condition (2). Let $u \in S$ and let $a \in \mathrm{C}_{0}\left(\mathcal{L}^{0}\right)$. Then for all $f \cdot \xi \in B_{u^{*}}$ we have

$$
\beta(u) a \beta(u)^{*}(f \cdot \xi)=\alpha(u)\left(a \alpha\left(u^{*}\right)(f)\right) \cdot \xi=\alpha(u)(a) f \cdot \xi .
$$

Furthermore, the map $\beta: S \rightarrow I(\mathcal{F})$ is a semigroup homomorphism since $\alpha: S \rightarrow$ $I\left(\mathrm{C}_{0}\left(\mathcal{L}^{0}\right)\right)$ is a semigroup homomorphism.

To prove the converse, let $(\beta, \mathcal{F})$ be a covariant representation of the pair $\left(S, \mathcal{L}^{0}\right)$. By [16, Theorem 9.8], $\mathrm{C}^{*}(\mathcal{L}) \cong \mathrm{C}_{0}\left(\mathcal{L}^{0}\right) \rtimes S$. We define an action of $\mathrm{C}^{*}(\mathcal{L})$ on $\mathcal{F}$ as follows. Let $f \in \mathfrak{S}(\mathcal{L})$. Then there is a natural number $n \in \mathbb{N}$ such that $f=$ $\left.\sum_{k=1}^{n} f_{k} \circ s\right|_{u_{k}}$ for bisections $u_{1}, \ldots, u_{n} \in S$ and $f_{k} \in \mathrm{C}_{c}\left(u_{k}^{*} u_{k}\right)$ for $k=1, \ldots, n$. We set

$$
\varphi(f)(\xi)=f \cdot \xi:=\sum_{k=1}^{n} \beta\left(u_{k}\right) \varphi_{\mathrm{C}_{0}\left(\mathcal{L}^{0}\right)}\left(f_{k}\right)(\xi) .
$$




\section{Actions of Ore monoids}

Equation (3.47) gives a well defined map $\bigoplus_{u \in S} \mathrm{C}_{\mathrm{c}}(u) \rightarrow \mathbb{B}(\mathcal{F})$. For the map $\varphi$ to extend to a map $\mathfrak{S}(\mathcal{L}) \rightarrow \mathbb{B}(\mathcal{F})$, it has to vanish on the kernel of the natural map $E: \bigoplus_{u \in S} \mathrm{C}_{\mathrm{c}}(u) \rightarrow \mathfrak{S}(\mathcal{L})$. By [10, Proposition B.2], the kernel of $E$ is given by the closed linear span of the set of elements of the form $f \delta_{u}-f \delta_{v}$ for $f \in \mathrm{C}_{\mathrm{c}}(u)$, $u, v \in S$ and $u \subset v$, where $\delta_{u}$ is the characteristic function on $u$. Let $f \delta_{u}-f \delta_{v}$ be such an element. Then $f=\left.g \circ s\right|_{u}$ with $g \in \mathrm{C}_{\mathrm{c}}\left(u^{*} u\right)$. We have $\beta(u)=\left.\beta(v)\right|_{\mathcal{F}_{u}}$ since $u \subset v$ and $(\beta, \mathcal{F})$ is a representation. Hence

$$
\left(f \delta_{u}-f \delta_{v}\right) \cdot \xi=\beta(u) \varphi_{\mathrm{C}_{0}\left(\mathcal{L}^{0}\right)}(g)(\xi)-\beta(v) \varphi_{\mathrm{C}_{0}\left(\mathcal{L}^{0}\right)}(g)(\xi)=0
$$

for all $\xi \in \mathcal{F}$. It follows that $\varphi\left(f \delta_{u}-f \delta_{v}\right)=0$. Since $\varphi$ is continuous, it vanishes on the kernel of $E$ and gives a well-defined map $\mathfrak{S}(\mathcal{L}) \rightarrow \mathbb{B}(\mathcal{F})$. This map extends to a non-degenerate ${ }^{*}$-homomorphism $\varphi_{\mathrm{C}^{*}(\mathcal{L})}: \mathrm{C}^{*}(\mathcal{L}) \rightarrow \mathbb{B}(\mathcal{F})$. The map $\varphi_{\mathrm{C}^{*}(\mathcal{L})}$ restricts to $(\beta, \mathcal{F})$ by construction. This finishes the proof.

Let $X$ be a locally compact Hausdorff space. Let $\mathcal{L}$ be a groupoid acting on $X$. Let $S \subset \operatorname{Bis}(\mathcal{L})$ be such that $S \ltimes \mathcal{L}^{0} \cong \mathcal{L}$. Then the action of $\mathcal{L}$ on $X$ is equivalent to an action of $S$ on $X$ by partial homeomorphisms, see [9, Theorem 3.7]. The action of $S$ is given as follows. Let $e \in S$ be an idempotent. Let $X_{e}:=r^{-1}(e)$. Now for each $u \in S$, define $\alpha_{u}: X_{u^{*} u} \rightarrow X_{u u^{*}}$ by

$$
\alpha_{u}(x)=g \cdot x,
$$

where $g \in u$ is the unique element with $r(x)=s(g)$.

Lemma 3.35. Let $X$ be a locally compact Hausdorff space. Let $\mathcal{L}$ be a groupoid acting on $X$. Let $S \subset \operatorname{Bis}(\mathcal{L})$ be a wide inverse semigroup. Let $D$ be a $\mathrm{C}^{*}$-algebra. There is a bijection between the set of correspondences $\mathcal{F}: \mathrm{C}^{*}(\mathcal{L} \ltimes X) \rightarrow D$ and the set of non-degenerate covariant representations of $(S, X)$.

Proof. The inverse semigroup $S$ is wide in the groupoid $\mathcal{L} \ltimes X$. Hence the claim follows from Proposition 3.34.

Theorem 3.36. The product systems $\left(\mathrm{C}^{*}\left(\tilde{\mathcal{X}}_{p}\right), \tilde{\mu}_{p, q}\right)$ and $\left(\mathrm{C}^{*}\left(\mathcal{X}_{p}\right), \mu_{p, q}\right)$ have the same colimits in $\mathfrak{C o r r}$ and in $\mathfrak{C o r r}_{\text {prop. }}$. The groupoid $\mathcal{H}$ is a groupoid model for the Cuntz-Pimsner algebra of the product system $\left(\mathrm{C}^{*}\left(\mathcal{X}_{p}\right), \mu_{p, q}\right)$.

Proof. Let $D$ be a $\mathrm{C}^{*}$-algebra and let $\left(\mathcal{F}, \tilde{V}_{p}\right)$ be a transformation from the diagram $\left(\mathrm{C}^{*}\left(\tilde{\mathcal{X}}_{p}\right), \tilde{\mu}_{p, q}\right)$ to const $_{D}$. Here $\mathcal{F}: \mathrm{C}^{*}(\tilde{\mathcal{G}}) \rightarrow \mathrm{C}^{*}(\mathcal{D})$ is a correspondence and $\tilde{V}_{p}: \mathrm{C}^{*}\left(\mathcal{X}_{p}\right) \otimes_{\mathrm{C}^{*}(\tilde{\mathcal{G}})} \mathcal{F} \rightarrow \mathcal{F}$ is an isomorphism for all $p \in P$; the isomorphisms $\tilde{V}_{p}$ satisfy condition (2.32).

Now the natural (left) action of $\mathcal{G}$ on $\tilde{\mathcal{G}}$ commutes with the right action of $\tilde{\mathcal{G}}$ on itself by multiplication. Hence $\tilde{\mathcal{G}}: \mathcal{G} \rightarrow \tilde{\mathcal{G}}$ is a correspondence (an actor in fact). It 
is proper since $r_{*}: \tilde{\mathcal{G}} / \tilde{\mathcal{G}}=\mathcal{H}^{0} \rightarrow \mathcal{G}^{0}$ is proper by Lemma 3.25 ; and hence it induces a non-degenerate ${ }^{*}$-homomorphism $\iota: \mathrm{C}^{*}(\mathcal{G}) \rightarrow \mathrm{C}^{*}(\tilde{\mathcal{G}})$.

We define

$$
\nu_{p}: \tilde{\mathcal{X}}_{p} \rightarrow \mathcal{X}_{p} \circ_{\mathcal{G}} \tilde{\mathcal{G}}, \quad\left(x_{p}, \xi\right) \mapsto\left[x_{p}, \xi\right] .
$$

The map $\nu_{p}$ is injective and $\mathcal{G}$-equivariant. Let $x=\left[x_{p},(g, \xi)\right] \in \mathcal{X}_{p} \circ_{\mathcal{G}} \tilde{\mathcal{G}}$, then $x=$ $\left[x_{p} g, \xi\right]=\nu_{p}\left(x_{p} g, \xi\right)$. Hence $\nu_{p}$ is surjective. Let $(g, \eta) \in \tilde{\mathcal{G}}$ and $\left(x_{p}, \xi\right) \in \tilde{\mathcal{X}}_{p}$ satisfy $g \eta=\xi$. Then

$$
\nu_{p}\left(x_{p}, \xi\right)(g, \eta)=\left[x_{p}, \xi\right](g, \eta)=\left[x_{p} g, \eta\right]=\left[x_{p} g, g^{-1} \xi\right] .
$$

Therefore, $\nu_{p}$ is $\tilde{\mathcal{G}}$-equivariant. It is also open and continuous. Hence it is an isomorphism. Thus its image under the functor $\mathfrak{G} \mathfrak{r} \rightarrow \mathfrak{C o r r}$ is an isomorphism

$$
\mathrm{C}^{*}\left(\tilde{\mathcal{X}}_{p}\right) \cong \mathrm{C}^{*}\left(\mathcal{X}_{p}\right) \otimes_{\mathrm{C}^{*}(\mathcal{G})} \mathrm{C}^{*}(\tilde{\mathcal{G}}) .
$$

As a result, we obtain isomorphisms

$$
\mathrm{C}^{*}\left(\mathcal{X}_{p}\right) \otimes_{\mathrm{C}^{*}(\mathcal{G})} \mathcal{F} \cong \mathrm{C}^{*}\left(\mathcal{X}_{p}\right) \otimes_{\mathrm{C}^{*}(\mathcal{G})} \mathrm{C}^{*}(\tilde{\mathcal{G}}) \otimes_{\mathrm{C}^{*}(\tilde{\mathcal{G}})} \mathcal{F} \cong \mathrm{C}^{*}\left(\tilde{\mathcal{X}}_{p}\right) \otimes_{\mathrm{C}^{*}(\tilde{\mathcal{G}})} \mathcal{F} \cong \mathcal{F} .
$$

Let $V_{p}: \mathrm{C}^{*}\left(\mathcal{X}_{p}\right) \otimes_{\mathrm{C}^{*}(\mathcal{G})} \mathcal{F} \rightarrow \mathcal{F}$ for $p \in P$ be the product of the isomorphisms in (3.49). Since $\tilde{V}_{p}$ satisfies condition (2.27), the isomorphisms $V_{p}$ also satisfy (2.27). This implies that $\left(\mathcal{F}, V_{p}\right)$ is a transformation from $\left(\mathrm{C}^{*}\left(\mathcal{X}_{p}\right), \mu_{p, q}\right)$ to the constant diagram const $_{D}$.

Conversely, let $\left(\mathcal{F}, V_{p}\right)$ be a transformation from $\left(\mathrm{C}^{*}\left(\mathcal{X}_{p}\right), \mu_{p, q}\right)$ to the constant diagram $\operatorname{const}_{D}$. We want to construct a transformation from $\left(\mathrm{C}^{*}\left(\tilde{\mathcal{X}}_{p}\right), \tilde{\mu}_{p, q}\right)$ to const $_{D}$. The space $\mathcal{X}_{p}$ is a correspondence from the space $\mathcal{X}_{p} / \mathcal{G}$ to $\mathcal{G}$, where the left anchor map $r=\mathrm{p}_{p}: \mathcal{X}_{p} \rightarrow \mathcal{X}_{p} / \mathcal{G}$ is the quotient map. The image of $\mathcal{X}_{p}: \mathcal{X}_{p} / \mathcal{G} \rightarrow \mathcal{G}$ under the functor $\mathfrak{G r} \rightarrow \mathfrak{C o r r}$ is a $\mathrm{C}^{*}$-correspondence $\mathrm{C}^{*}\left(\mathcal{X}_{p}\right): \mathrm{C}_{0}\left(\mathcal{X}_{p} / \mathcal{G}\right) \rightarrow \mathrm{C}^{*}(\mathcal{G})$. That is, the $\mathrm{C}^{*}$-algebra $\mathrm{C}_{0}(\mathcal{X} / \mathcal{G})$ acts on the Hilbert $\mathrm{C}^{*}(\mathcal{G})$-module $\mathrm{C}^{*}\left(\mathcal{X}_{p}\right)$ by pointwise multiplication. The Hilbert $D$-modules $\mathrm{C}^{*}\left(\mathcal{X}_{p}\right) \otimes_{\mathrm{C}^{*}(\mathcal{G})} \mathcal{F}$ and $\mathcal{F}$ are isomorphic via the map $V_{p}$. Hence we may view $\mathcal{F}$ as a $\mathrm{C}^{*}$-correspondence from $\mathrm{C}_{0}\left(\mathcal{X}_{p} / \mathcal{G}\right)$ to $D$. The left action of $\mathrm{C}_{0}\left(\mathcal{X}_{p} / \mathcal{G}\right)$ on $\mathcal{F}$ is given by $\varphi_{\mathrm{C}_{0}\left(\mathcal{X}_{p} / \mathcal{G}\right)}(a):=$ $V_{p} a V_{p}^{-1}$. Furthermore, for all $p, q \in P$, the map $r_{p, q}: \mathcal{X}_{p q} / \mathcal{G} \rightarrow \mathcal{X}_{p} / \mathcal{G}$ is proper by Lemma 3.25. Hence it induces a map $r_{p, q}^{*}: \mathrm{C}_{0}\left(\mathcal{X}_{p} / \mathcal{G}\right) \rightarrow \mathrm{C}_{0}\left(\mathcal{X}_{p q} / \mathcal{G}\right)$. We claim that

$$
\varphi_{\mathrm{C}_{0}\left(\mathcal{X}_{p q} / \mathcal{G}\right)} r_{p, q}^{*}=\varphi_{\mathrm{C}_{0}\left(\mathcal{X}_{p} / \mathcal{G}\right)} .
$$

First, we have

$$
V_{p q}=V_{p}\left(\operatorname{id}_{\mathrm{C}^{*}\left(\mathcal{X}_{p}\right)} \otimes_{\mathrm{C}^{*}(\mathcal{G})} V_{q}\right)\left(u_{p, q}^{-1} \otimes_{\mathrm{C}^{*}\left(\mathcal{X}_{p}\right)} \mathrm{id}_{\mathcal{F}}\right) .
$$


Hence

$$
V_{p q}^{-1}=\left(u_{p, q} \otimes_{\mathrm{C}^{*}(\mathcal{G})} \operatorname{id}_{\mathcal{F}}\right)\left(\operatorname{id}_{\mathrm{C}^{*}\left(\mathcal{X}_{p}\right)} \otimes_{\mathrm{C}^{*}(\mathcal{G})} V_{q}^{-1}\right) V_{p}^{-1}
$$

We also have

$$
\left(u_{p, q}^{-1} \otimes_{\mathrm{C}^{*}(\mathcal{G})} \operatorname{id}_{\mathcal{F}}\right)\left(r_{p, q}^{*}(a)\right)\left(u_{p, q} \otimes_{\mathrm{C}^{*}\left(\mathcal{X}_{p}\right)} \operatorname{id}_{\mathcal{F}}\right)=a \otimes_{\mathrm{C}^{*}(\mathcal{G})} \operatorname{id}_{\mathrm{C}^{*}\left(\mathcal{X}_{q}\right)} \otimes_{\mathrm{C}^{*}(\mathcal{G})} \operatorname{id}_{\mathcal{F}} .
$$

So

$$
\begin{aligned}
\varphi_{\mathrm{C}_{0}\left(\mathcal{X}_{p q} / \mathcal{G}\right)} r_{p, q}^{*}(a)= & V_{p q}\left(a \otimes_{\mathrm{C}^{*}(\mathcal{G})} \operatorname{id}_{\mathcal{F}}\right) V_{p q}^{-1} \\
= & V_{p}\left(\operatorname{id}_{\mathrm{C}^{*}\left(\mathcal{X}_{p}\right)} \otimes_{\mathrm{C}^{*}(\mathcal{G})} V_{q}\right)\left(a \otimes_{\mathrm{C}^{*}(\mathcal{G})} \operatorname{id}_{\mathrm{C}^{*}\left(\mathcal{X}_{q}\right)} \otimes_{\mathrm{C}^{*}(\mathcal{G})} \operatorname{id}_{\mathcal{F}}\right) . \\
& \left(\operatorname{id}_{\mathrm{C}^{*}\left(\mathcal{X}_{p}\right)} \otimes_{\mathrm{C}^{*}(\mathcal{G})} V_{q}^{-1}\right) V_{p}^{-1} \\
= & V_{p}\left(a \otimes_{\mathrm{C}^{*}(\mathcal{G})} \operatorname{id}_{\mathcal{F}}\right) V_{p}^{-1}=\varphi_{\mathrm{C}_{0}\left(\mathcal{X}_{p} / \mathcal{G}\right)}(a) .
\end{aligned}
$$

And the claim follows. Equation (3.50) and the universal property of the inductive limit imply that $\left(\varphi_{\mathrm{C}_{0}\left(\mathcal{X}_{p} / \mathcal{G}\right)}\right)_{p \in P}$ induces a map

$$
\varphi: \underset{\operatorname{Cim}_{P}}{\lim _{0}}\left(\mathrm{C}_{0}\left(\mathcal{X}_{p} / \mathcal{G}\right), r_{p, q}^{*}\right) \rightarrow \mathbb{B}(\mathcal{F}) .
$$

This map is non-degenerate since $\varphi_{\mathrm{C}_{0}\left(\mathcal{X}_{p} / \mathcal{G}\right)}$ is non-degenerate for all $p \in P$. Furthermore,

$$
\underset{\overrightarrow{\mathcal{C}_{P}}}{\lim }\left(\mathrm{C}_{0}\left(\mathcal{X}_{p} / \mathcal{G}\right), r_{p, q}^{*}\right)=\mathrm{C}_{0}\left(\underset{\overleftarrow{\mathcal{C}_{P}}}{\lim }\left(\mathcal{X}_{p} / \mathcal{G}, r_{p, q}\right)\right)=\mathrm{C}_{0}\left(\mathcal{H}^{0}\right)
$$

Summing up, we obtain a $\mathrm{C}^{*}$-correspondence $\mathcal{F}: \mathrm{C}_{0}\left(\mathcal{H}^{0}\right) \rightarrow \mathcal{D}$. And $\mathcal{F}$ is a correspondence $\mathrm{C}^{*}(\mathcal{G}) \rightarrow \mathcal{D}$. We have to show that $\mathcal{F}$ extends to a correspondence $\mathcal{F}: \mathrm{C}^{*}(\tilde{\mathcal{G}})=\mathrm{C}^{*}\left(\mathcal{G} \ltimes \mathcal{H}^{0}\right) \rightarrow \mathcal{D}$. By Proposition 3.34, the correspondence $\mathcal{F}: \mathrm{C}^{*}(\mathcal{G}) \rightarrow \mathcal{D}$ is equivalent to a non-degenerate covariant representation $(\alpha, \mathcal{F})$ of the pair $\left(S, \mathcal{G}^{0}\right)$. We will show that the pair $(\alpha, \mathcal{F})$, where $\mathcal{F}$ is now a correspondence from $\mathrm{C}_{0}\left(\mathcal{H}^{0}\right)$ to $D$, is also a covariant representation. Then using Proposition 3.34 again we get a correspondence $\mathcal{F}: \mathrm{C}^{*}(\tilde{\mathcal{G}}) \rightarrow D$. First, linearity of the maps $V_{p}$ for $p \in P$ implies condition (1) in Definition 3.33. Secondly, the action of $\mathcal{G}$ on $\mathcal{X}_{p} / \mathcal{G}$ induces an action of $S$ on $\mathcal{X}_{p} / \mathcal{G}$. The resulting action induces an action of $S$ on $\mathrm{C}_{0}\left(\mathcal{X}_{p} / \mathcal{G}\right)$. With a slight abuse of notation, we denote this action also by $\alpha$. The action is given by

$$
\alpha_{u}\left(f_{p}\left(x_{p}\right)\right)=f_{p}\left[g \cdot x_{p}\right]
$$

for all $f_{p} \in \mathfrak{S}\left(\mathcal{X}_{p} / \mathcal{G}\right), u \in S$ and $x_{p} \in \mathcal{G}$, where $g \in u$ is the unique element with $s(g)=r\left(x_{p}\right)$. This action commutes, as expected, with the maps 
$r_{p, q}^{*}: \mathrm{C}_{0}\left(\mathcal{X}_{p} / \mathcal{G}\right) \rightarrow \mathrm{C}_{0}\left(\mathcal{X}_{p q} / \mathcal{G}\right)$ for all $p, q \in \mathcal{G}$ and induces the action of $S$ on $\mathrm{C}_{0}\left(\mathcal{H}^{0}\right)$. We only need to check that the condition (2) in Definition 3.33 holds for the pairs $\left(S, \mathrm{C}_{0}\left(\mathcal{X}_{p} / \mathcal{G}\right)\right)$ for all $p \in P$. Now let $p \in P$. Let $u \in S, f_{p} \in \mathrm{C}_{c}\left(\mathcal{X}_{p} / \mathcal{G}\right)$. Then for all $\xi_{p} \otimes \xi \in \mathrm{C}^{*}\left(\mathcal{X}_{p}\right) \otimes_{\mathrm{C}^{*}(\mathcal{G})} \mathcal{F}$ and all $f \in \mathfrak{S}(u)$, we have

$$
\beta(u) f_{p} \beta\left(u^{*}\right)\left(V_{p}\left(f \xi_{p} \otimes \xi\right)\right)=\beta(u) V_{p}\left(f_{p} \alpha\left(u^{*}\right)(f) \xi_{p} \otimes \xi\right) .
$$

But for all $x_{p} \in \mathcal{X}_{p}$ we have

$$
f_{p} \alpha\left(u^{*}\right)(f) \xi_{p}\left(x_{p}\right)=f_{p}\left[x_{p}\right] f\left(g^{-1}\right) \xi_{p}\left(g^{-1} x_{p}\right),
$$

where $g \in u$ is the unique element with $r(g)=r_{p}\left(x_{p}\right)$. It follows that

$$
f_{p} \alpha\left(u^{*}\right)(f) \xi_{p}=\alpha(u)\left(f_{p}\right) \alpha\left(u^{*}\right)(f) \xi_{p} .
$$

Thus

$$
\beta(u) f_{p} \beta\left(u^{*}\right)\left(V_{p}\left(f \xi_{p} \otimes \xi\right)\right)=V_{p}\left(\alpha(u)\left(f_{p}\right) f \xi_{p} \otimes \xi\right)=\alpha(u)\left(f_{p}\right) V_{p}\left(f \xi_{p} \otimes \xi\right) .
$$

This implies that the condition (2) is satisfied. Therefore, we get a correspondence $\mathcal{F}: \mathrm{C}^{*}(\tilde{\mathcal{G}}) \rightarrow D$. Furthermore, equality (3.49) holds. Hence we have a transformation $\left(\mathcal{F}, \tilde{V}_{p}\right)$ from $\left(\mathrm{C}^{*}\left(\tilde{\mathcal{X}}_{p}\right), \tilde{\mu}_{p, q}\right)$ to const $_{D}$. Clearly, the transformation from $\left(\mathrm{C}^{*}\left(\mathcal{X}_{p}\right), \mu_{p, q}\right)$ to const $_{D}$ obtained as before from $\left(\mathcal{F}, \tilde{V}_{p}\right)$ is again $\left(\mathcal{F}, V_{p}\right)$.

Finally, let $\left(\mathcal{F}, \tilde{V}_{p}\right)$ be a transformation from $\left(\mathrm{C}^{*}\left(\tilde{\mathcal{X}}_{p}\right), \tilde{\mu}_{p, q}\right)$ to const $_{D}$. Let $(\beta, \mathcal{F})$ be the representation of $\left(S, \mathcal{G}^{0}\right)$ obtained as before. Let $u$ be a bisection in $\mathcal{X}_{p}$ for $p \in P$ and let $f \in \mathrm{C}_{0}\left(u^{*} u\right)$. Then $\left.f \circ s\right|_{u} \in \mathrm{C}_{0}(u) \subseteq \mathrm{C}_{0}\left(\mathcal{X}_{p} / \mathcal{G}\right)$. So

$$
\varphi\left(\left.f \circ s\right|_{u}\right)=\beta(u) \varphi(f) .
$$

The functions of the form $\left.f \circ s\right|_{u}$ span a dense subset in $\mathrm{C}_{0}\left(\mathcal{X}_{p} / \mathcal{G}\right)$. Hence the action of $\mathrm{C}_{0}\left(\mathcal{X}_{p} / \mathcal{G}\right)$ is given by the action of $\mathrm{C}_{0}\left(\mathcal{G}^{0}\right)$ on $\mathcal{F}$ and the isomorphisms $V_{p}$ constructed from the action as above. Thus $\left(\mathcal{F}, \tilde{V}_{p}\right)$ is determined uniquely by the transformation from $\left(\mathrm{C}^{*}\left(\mathcal{X}_{p}\right), \mu_{p, q}\right)$ to const $_{D}$ obtained from it.

\subsection{Examples}

\subsubsection{Actions by local homeomorphisms}

Let $X$ be a locally compact Hausdorff space. Let $P$ be an Ore monoid acting on $X$ by local homeomorphisms. We can turn this action into an action by correspondences on the space $X$ in two different ways. First, for all $p \in P$ we may form the correspondence $X_{p}$ as a copy of the space $X$ with source and range maps given by

$$
s_{p}:=p: X \rightarrow X, \quad r_{p}:=\mathrm{id}: X \rightarrow X .
$$




\section{Actions of Ore monoids}

The map $\sigma_{p, q}: X \times_{s_{p}, X, r_{q}} X,(x, p(x)) \mapsto x$, is a correspondence isomorphism. Clearly, the isomorphisms $\sigma_{p, q}$ satisfy the coherence condition (3.1). Second, we may take the dual $X_{p}^{*}$ of $X_{p}$. That is, $X_{p}^{*}=X$ as a topological space and the source and range maps are given by

$$
s_{p}:=\mathrm{id}: X \rightarrow X, \quad r_{p}:=p: X \rightarrow X .
$$

Similarly, the map $\sigma_{p, q}^{*}: X \times_{s_{p}, X, r_{q}} X,(q(x), x) \mapsto x$, is a correspondences isomorphism.

What are the colimits of the diagrams $\left(X_{p}, \sigma_{p, q}\right)$ and $\left(X_{p}^{*}, \sigma_{p, q}^{*}\right)$ in $\mathfrak{G r}$ ?

(a) We start with the diagram $\left(X_{p}, \sigma_{p, q}\right)$. This diagram consists of tight correspondences, which allows us to apply the method in Section 3.2 to get the colimit. Let $p, q, k \in P$. Then the map $\alpha_{p, q}^{k}: X_{p} \times_{s_{p}, X, s_{q}} X_{q} \rightarrow X_{p k} \times_{s_{p k}, X, s_{q k}} X_{q k}$ is the identity map. It follows that for all $g \in G=G[P]$ the inductive limit space $\mathcal{H}_{g}$ can be described by

$$
\begin{aligned}
\mathcal{H}_{g} & =\bigsqcup_{(p, q) \in R_{g}} X_{p} \times_{s_{p}, X, s_{q}} X_{q} \\
& =\left\{(x, p, q, y) \in X \times P \times P \times X \mid p q^{-1}=g, p(x)=q(y)\right\} .
\end{aligned}
$$

The groupoid $\mathcal{H}$ is the disjoint union of all $\mathcal{H}_{g}$. Therefore, as a space, $\mathcal{H}$ can be written as

$$
\mathcal{H}=\left\{(x, g, y) \in X \times G \times X \mid \exists p_{1}, p_{2} \in P, g=p_{1} p_{2}^{-1}, p_{1}(x)=p_{2}(y)\right\} .
$$

The groupoid structure on $\mathcal{H}$ is given by

$$
\left(x, p_{1} p_{2}^{-1}, y\right)\left(y, p_{2} p_{3}^{-1}, z\right)=\left(x, p_{1} p_{3}^{-1}, z\right), \quad\left(u, p q^{-1}, v\right)^{-1}=\left(v, q p^{-1}, u\right)
$$

for all $\left(x, p_{1} p_{2}^{-1}, y\right),\left(y, p_{2} p_{3}^{-1}, z\right),\left(u, p q^{-1}, v\right) \in \mathcal{H}$. That is, the groupoid $\mathcal{H}$ is the transformation groupoid associated with the action of $P$ on $X$.

(b) We describe the colimit of the diagram $\left(X_{p}^{*}, \sigma_{p, q}^{*}\right)$. For this to be a diagram of proper correspondences, we need to assume from now on that the local homeomorphisms $p: X \rightarrow X$ are proper for all $p \in P$. We also associated in [1] a groupoid to an action of an Ore monoid by topological correspondences. We will show that the groupoid constructed in [1] is isomorphic to the colimit of the diagram $\left(X_{p}^{*}, \sigma_{p, q}^{*}\right)$ in $\mathfrak{G r}$. First, the projection map $r_{p, q}: X_{p q} \rightarrow X_{p}$ is given by $r_{p, q}(x)=q(x)$ for all $x \in X_{p q}$. The unit space of the colimit groupoid is the inverse limit of $\left(X_{p q}, r_{p, q}\right)$ over the filtered category $\mathcal{C}_{P}$. That is, $\mathcal{H}^{0}=\lim _{\mathcal{C}_{P}}\left(X_{p q}, q\right)$. That is, a point $x \in \mathcal{H}^{0}$ is given by $\left(x_{p}\right)_{p \in P}$ with 
$q\left(x_{p q}\right)=x_{p}$. Elements of $\mathcal{H}^{0}$ are called complete histories. Moreover, the tight correspondences $\tilde{X}_{p}^{*}$ for $p \in P$ are given by

$$
\tilde{X}_{p}^{*}=X_{p}^{*} \times_{s_{p}, X, r} \mathcal{H}^{0}=\left\{\left(x,\left(x_{p}\right)\right) \in X_{p}^{*} \times \mathcal{H}^{0} \mid x=p\left(x_{p}\right)\right\} .
$$

The range map on $\tilde{X}_{p}^{*}$ is given by

$$
\tilde{r}_{p}\left(x_{p}, \eta\right)=x_{p} \cdot \eta
$$

Recall that, by Equation (3.33), we have

$$
\left(x_{p} \cdot \eta\right)_{p t}=\sigma_{p, t}^{-1}\left(x_{p}, \eta_{t}\right)=\eta_{t}
$$

for all $t \in P$. That is, the range map on a pair $\left(x_{p}, \eta\right)$ in $\mathcal{H}^{0}$ simply concatenates $x_{p}$ to the complete history $\eta$. The source map on $\tilde{X}_{p}^{*}$ is given by

$$
\tilde{s}_{p}\left(x_{p}, \eta\right)=\eta .
$$

To land back in the setting of [1], we transform the action $\left(\tilde{\mathcal{X}}_{p}, \tilde{\sigma}_{p, q}\right)$ to an action as in the previous case (a). The range map $\tilde{r}_{p}$ is a homeomorphism by Lemma 3.27. Therefore, we may replace $\tilde{X}_{p}^{*}$ by $\mathcal{H}^{0}$ using $\tilde{r}_{p}$. We also replace the source map $\tilde{s}_{p}$ by $\tilde{s} \circ \tilde{r}_{p}^{-1}$. The new tight correspondence is then $\tilde{X}_{p}^{*}=$ $\mathcal{H}^{0}: \mathcal{H}^{0} \rightarrow \mathcal{H}^{0}$, where the range map on $\tilde{X}_{p}^{*}$ is the identity and the source map $\tilde{s}_{p}$ is the local homeomorphism which takes a complete history $\eta$ and forgets what happens in the last time period of length $p$. Formally, $\tilde{s}_{p}(\eta)_{t}=$ $\eta_{p t}$ for $t \in P$. Finally, we replace the isomorphisms $\tilde{\sigma}_{p, q}^{*}: \tilde{X}_{p}^{*} \times_{\tilde{s}_{p}, \mathcal{H}^{0}, \tilde{r}_{q}} \tilde{X}_{q}^{*} \rightarrow$ $\tilde{X}_{p q}^{*}$ with the isomorphisms $\tilde{\sigma}_{p, q}^{*}: \mathcal{H}^{0} \times_{\tilde{s}_{p}, \mathcal{H}^{0} \text {,id }} \mathcal{H}^{0} \rightarrow \mathcal{H}^{0},\left(x, \tilde{s}_{p}(x)\right) \mapsto x$. The colimit groupoid $\mathcal{H}$ of the action $\left(\tilde{X}_{p}^{*}, \tilde{\sigma}_{p, q}^{*}\right)$ is then given by

$$
\mathcal{H}=\left\{(x, g, y) \in \mathcal{H}^{0} \times G \times \mathcal{H}^{0} \mid \exists p_{1}, p_{2} \in P, g=p_{1} p_{2}^{-1}, \tilde{s}_{p_{1}}(x)=\tilde{s}_{p_{2}}(y)\right\} .
$$

Thus, we obtain the transformation groupoid associated with the action of $P$ on $\mathcal{H}^{0}$ given by the local homeomorphism $\tilde{s}_{p}$, compare [1, Definition 4.12].

\subsubsection{Self-similar group actions}

In the following, we fix a self-similar group action $(G, X)$, see Example 2.23. We want to compute the groupoid model associated with the $\mathbb{N}$-action given by iterations of the (proper) correspondence $X \times G: G \rightarrow G$. First we show that the iterations $(X \times G)^{n}: G \rightarrow G$ are given by self-similarities $\left(G, X^{n}\right)$. The group $G$ acts on the space $X^{n}$ recursively by

$$
g(x w)=\left.g(x) g\right|_{x}(w)
$$


for all $g, h \in G, x \in X$ and all $w \in X^{n-1}$. And the cocycle $G \times X^{n} \rightarrow G$ is given by

$$
\left.g\right|_{x_{1} x_{2} \cdots x_{n}}=\left.\left.\left.g\right|_{x_{1}}\right|_{x_{2}} \cdots\right|_{x_{n}}
$$

This gives a self-similar group action $\left(G, X^{n}\right)$ and hence a correspondence $X^{n} \times$ $G: G \rightarrow G$.

Lemma 3.37. Let $(G, X)$ be a self-similar group and let $\mathcal{X}=X \times G$ be the associated correspondence. Then for all $n \in \mathbb{N}$ the $n$-fold composition $\mathcal{X}_{n}$ is isomorphic to the correspondence $X^{n} \times G: G \rightarrow G$.

Proof. The map $\psi_{n}: \mathcal{X}_{n} \rightarrow X^{n} \times G$ given by

$$
\begin{aligned}
& {\left[\left(\left(x_{1}, g_{1}\right),\left(x_{2}, g_{2}\right), \ldots\left(x_{n}, g_{n}\right)\right]\right)} \\
& \quad \mapsto\left(x_{1}, g_{1}\left(x_{2}\right), \ldots\left(\left.\left(\left.g_{1}\right|_{x_{2}} g_{2}\right)\right|_{x_{3}} \ldots g_{n-1}\right)\left(x_{n}\right),\left.\left(\left.\left(\left.g_{1}\right|_{x_{2}} g_{2}\right)\right|_{x_{3}} \ldots g_{n-1}\right)\right|_{x_{n}} g_{n}\right),
\end{aligned}
$$

is an isomorphism. This follows since the correspondences $\mathcal{X}_{n}$ and $X^{n} \times G$ are discrete and the map $\psi_{n}$ is a bijection and $G$ equivariant.

In [37], Nekrashevych associates a $\mathrm{C}^{*}$-algebra $\mathcal{O}_{G, X}$ to a self-similar group $(G, X)$ and in $[37, \S 5.2]$ he associates a groupoid model $\mathcal{D}_{G}$ to $\mathcal{O}_{G, X}$. The groupoid $\mathcal{D}_{G}$ is given as follows. Let $\langle G, X\rangle$ be the quotient of the free inverse semigroup generated by the set $\left\{S_{x}, S_{x}^{*} \mid x \in X\right\} \cup G$ under the relations

(1) the relations in the group $G$;

(2) $S_{x}^{*} S_{x}=1$ for all $x \in X$ and $S_{x}^{*} S_{y}=0$ for $x \neq y$.

The inverse semigroup $\langle G, X\rangle$ acts on the space $X^{\omega}$ by the local homeomorphisms

$$
S_{u} g S_{v}^{*}(v \xi)=u g(\xi)
$$

The groupoid $\mathcal{D}_{G}$ is then the groupoid of germs of this action. In the theory of self-similar groups, the action of $G$ on $X^{*}$ is always assumed to be faithful; see [37]. Under this assumption, the germ groupoid is the same as the transformation groupoid of the action. Thus if the action of $G$ on $X^{*}$ is faithful, then the transformation groupoid $\langle G, X\rangle \ltimes X^{\omega}$ is always effective.

Theorem 3.38. Let $(G, X)$ be a self-similar group and let $\mathcal{X}=X \times G$ be the associated correspondence. Then $\langle G, X\rangle \ltimes X^{\omega}$ is isomorphic to the colimit $\mathcal{H}$ of the $\operatorname{diagram}(\mathbb{N},+) \rightarrow \mathfrak{G r}_{\text {prop }}$ associated with $\mathcal{X}$. If the action of $G$ on $X^{*}$ is faithful, then $\mathcal{D}_{G}$ is effective; it is isomorphic to $\mathcal{H}$. 
Proof. By Equation (3.29), the unit space $\mathcal{H}^{0}$ of the groupoid model $\mathcal{H}$ is

$$
\mathcal{H}^{0}=\lim _{\longleftarrow}\left(\mathcal{X}_{n} / G, r_{n, m}\right)
$$

where $r_{n, m}$ is the map

$$
\begin{aligned}
& r_{n, m}: \mathcal{X}^{n+m} / G \rightarrow \mathcal{X}^{n} / G \\
& \quad\left[\left(x_{1}, g_{1}\right),\left(x_{2}, g_{2}\right), \ldots,\left(x_{n+m}, g_{n+m}\right)_{n+m}\right] \mapsto\left[\left(x_{1}, g_{1}\right),\left(x_{2}, g_{2}\right), \ldots,\left(x_{n}, g_{n}\right)\right] .
\end{aligned}
$$

By Lemma 3.37, $\mathcal{X}_{n} / G$ is homeomorphic to the space $X^{n}$. This homeomorphism is equivariant with respect to the left $G$-actions on $\mathcal{X}_{n} / G$ and $X^{n}$. Under these homeomorphisms, the maps $r_{n, m}$ for $n, m \in \mathbb{N}$ correspond to the maps $X^{n+m} \rightarrow$ $X^{n},\left(x_{1} \ldots x_{n+m}\right) \mapsto\left(x_{1} \ldots x_{n}\right)$, which we also denote by $r_{n, m}$. Thus $\mathcal{H}^{0}$ is homeomorphic to

$$
X^{\omega}:=\lim _{\longleftarrow}\left(X^{n}, r_{n, m}\right) .
$$

The space $X^{\omega}$ is the space of right infinite words on the alphabet $X$ in the terminology of [37]. We identify $X^{\omega}$ with $\mathcal{H}^{0}$ in the sequel. The action of the group $G$ on the set of right infinite words $X^{\omega}=\mathcal{H}^{0}$ is obtained recursively from Equation (2.13). It is given by

$$
g \cdot\left(x_{1}, x_{2}, \ldots\right)=\left(g\left(x_{1}\right),\left.g\right|_{x_{1}}\left(x_{2}\right),\left.g\right|_{x_{1} x_{2}}\left(x_{3}\right), \ldots\right)
$$

for $g \in G$ and $x_{i} \in \mathcal{X}$. Next, let $n \in \mathbb{N}$. The tight correspondence $\tilde{\mathcal{X}}_{n}: G \ltimes X^{\omega} \rightarrow$ $G \ltimes X^{\omega}$ is given by $\tilde{\mathcal{X}}_{n}=X^{n} \times G \times X^{\omega}$. The source and range maps $\tilde{s}_{n}$ and $\tilde{r}_{n}$ are given by

$$
\begin{aligned}
& \tilde{s}_{n}\left(\left(x_{1}, x_{2}, \ldots, x_{n}, g\right), \eta\right)=\eta \\
& \tilde{r}_{n}\left(\left(x_{1}, x_{2}, \ldots, x_{n}, g\right), \eta\right)=\left(x_{1}, x_{2}, \ldots, x_{n}\right) \cdot(g \cdot \eta)
\end{aligned}
$$

for all $\left(x_{1}, x_{2}, \ldots, x_{n}, g, \eta\right) \in X^{n} \times G \times X^{\omega}$. That is, the range of an element $\left(x_{1}, x_{2}, \ldots, x_{n}, g, \eta\right) \in X^{n} \times G \times X^{\omega}$ is obtained by first acting with the group element $g$ on $\eta$ and then concatenating the finite word $\left(x_{1}, x_{2}, \ldots, x_{n}\right)$ to the resulting infinite path $g \cdot \eta$.

Let $n, m \in \mathbb{N}$. We have

$$
\begin{aligned}
& \tilde{\mathcal{X}}_{n}{ }^{\circ} G \ltimes X^{\omega} \tilde{\mathcal{X}}_{m}^{*}= \\
& \left\{[(\bar{x}, g, \xi),(\bar{y}, h, \xi)] \mid((\bar{x}, g, \xi),(\bar{y}, h, \xi)) \in\left(X^{n} \times G \times X^{\omega}\right) \times\left(X^{m} \times G \times X^{\omega}\right)\right\},
\end{aligned}
$$




\section{Actions of Ore monoids}

where the equivalence relation is given by

$$
((\bar{x}, g, \xi),(\bar{y}, h, \xi)) \sim\left(\left(\bar{x}, g t, t^{-1} \cdot \xi\right),\left(\bar{y}, h t, t^{-1} \cdot \xi\right)\right)
$$

for all $t \in G$. Thus $((\bar{x}, g, \xi),(\bar{y}, h, \xi)) \sim\left(\left(\bar{x}, g h^{-1}, h \cdot \xi\right),(\bar{y}, 1, h \cdot \xi)\right)$ in $\tilde{\mathcal{X}}_{n}{ }{ }_{G \ltimes X^{\omega}} \tilde{\mathcal{X}}_{m}^{*}$. Hence we can rewrite $\tilde{\mathcal{X}}_{n}{ }^{\circ}{ }_{G \ltimes X}{ }^{\omega} \tilde{\mathcal{X}}_{m}^{*}$ as the equivalence classes of quadruples $(\bar{x}, g, \bar{y}, \xi)$ in the space $X^{n} \times G \times X^{m} \times X^{\omega}$ under the equivalence relation

$$
(\bar{x}, g, \bar{y}, \xi) \sim\left(\bar{x}, g h^{-1}, \bar{y}, h \xi\right)
$$

for all $h \in G$. If $k \in \mathbb{N}$, then the map $\alpha_{n, m}^{k}$ is given by

$$
\alpha_{n, m}^{k}([\bar{x}, g, \bar{y}, \xi])=\left[\bar{x} \cdot g(\bar{z}),\left.g\right|_{\bar{z}}, \bar{y} \bar{z}, \eta\right]
$$

for all $[\bar{x}, g, \bar{y}, \xi] \in \tilde{\mathcal{X}}_{n}{ }^{\circ} G \ltimes X^{\omega} \tilde{\mathcal{X}}_{m}^{*}$, where $\eta \in X^{\omega}$ and $\bar{z} \in X^{k}$ are such that $\bar{z} \eta=\xi$.

Finally, the groupoid $\mathcal{H}$ as a set is given by

$$
\mathcal{H}=\left\{[\bar{x}, g, \bar{y}, \xi] \mid(\bar{x}, g, \bar{y}, \xi) \in X^{n} \times G \times X^{m} \times X^{\omega} \text { for some } n, m \in \mathbb{N}\right\} .
$$

The unit space of $\mathcal{H}$ is $X^{\omega}$ and the source and range maps on $\mathcal{H}$ are

$$
s([\bar{x}, g, \bar{y}, \xi])=\bar{y} \xi, \quad r([\bar{x}, g, \bar{y}, \xi])=\bar{x} \cdot g(\xi)
$$

for all $[\bar{x}, g, \bar{y}, \xi] \in \mathcal{H}$. The inverse of an element $[\bar{x}, g, \bar{y}, \xi] \in \mathcal{H}$ is

$$
([\bar{x}, g, \bar{y}, \xi])^{-1}=\left[\bar{y}, g^{-1}, \bar{x}, g(\xi)\right] .
$$

If $([\bar{x}, g, \bar{y}, \xi],[\bar{y}, h, \bar{z}, \eta]) \in \mathcal{H}^{2}$ with $\bar{x} \in X^{n}, \bar{y} \in X^{m}$ and $\bar{z} \in X^{k}$ then

$$
[\bar{x}, g, \bar{y}, \xi] \cdot[\bar{y}, h, \bar{z}, \eta]=[\bar{x}, g h, \bar{z}, \eta] .
$$

Since $([\bar{x}, g, \bar{y}, \xi],[\bar{y}, h, \bar{z}, \eta]) \in \mathcal{H}^{2}$, we have $\bar{y} \xi=\bar{y} \cdot h(\eta)$. So $\xi=h(\eta)$.

We check that the map

$$
\psi: \mathcal{H} \rightarrow \mathcal{D}_{G}, \quad[\bar{x}, g, \bar{y}, \xi] \mapsto\left[S_{\bar{x}} g S_{\bar{y}}^{*}, \bar{y} \cdot \xi\right],
$$

is an isomorphism. Let $[\bar{x}, g, \bar{y}, \xi] \in \mathcal{H}$. Then

$$
\begin{aligned}
\psi\left([\bar{x}, g, \bar{y}, \xi]^{-1}\right) & =\psi\left[\bar{y}, g^{-1}, \bar{x}, g(\xi)\right] \\
& =\left[S_{\bar{y}} g^{-1} S_{\bar{x}}^{*}, \bar{x} \cdot g(\xi)\right]=\left[S_{\bar{x}} g S_{\bar{y}}^{*}, \bar{y} \cdot \xi\right]^{-1}=\psi([\bar{x}, g, \bar{y}, \xi])^{-1}
\end{aligned}
$$

If $([\bar{x}, g, \bar{y}, \xi],[\bar{y}, h, \bar{z}, \eta]) \in \mathcal{H}^{2}$ with $\bar{x} \in X^{n}, \bar{y} \in X^{m}$ and $\bar{z} \in X^{k}$ then

$$
\begin{aligned}
\psi([\bar{x}, g, \bar{y}, \xi]) \cdot \psi([\bar{y}, h, \bar{z}, \eta]) & =\left[S_{\bar{x}} g S_{\bar{y}}^{*}, \bar{y} \xi\right] \cdot\left[S_{\bar{y}} h S_{\bar{z}}^{*}, \bar{z} \eta\right] \\
& =\left[S_{\bar{x}} g S_{\bar{y}}^{*} \cdot S_{\bar{y}} h S_{\bar{z}}^{*}, \bar{z} \eta\right]=\left[S_{\bar{x}} g h S_{\bar{z}}^{*}, \bar{z} \eta\right] \\
& =[\bar{x}, g h, \bar{z}, \eta]=\psi([\bar{x}, g, \bar{y}, \xi] \cdot[\bar{y}, h, \bar{z}, \eta]) .
\end{aligned}
$$


This implies that $\psi$ is a groupoid homomorphism. Let $a=\left[S_{\bar{x}} g S_{\bar{y}}^{*}, \xi\right] \in \mathcal{D}_{G}$. Then since $\xi$ is in the domain of the local homeomorphism $S_{\bar{x}} g S_{\bar{y}}^{*}$, it is of the form $\bar{y} \eta$ for $\eta \in X^{\omega}$. Then $[\bar{x}, g, \bar{y}, \eta] \in \mathcal{H}$ and $\psi([\bar{x}, g, \bar{y}, \eta])=a$. Hence $\psi$ is surjective. Let $[\bar{x}, g, \bar{y}, \eta] \in \mathcal{H}$ with $\psi\left([\bar{x}, g, \bar{y}, \eta]=\left[S_{\bar{x}} g S_{\bar{y}}^{*}, \bar{y} \cdot \eta\right] \in \mathcal{D}_{G}^{0}\right.$. This implies that there is an open set $V=\bar{y} U \subset X^{\omega}$ such that the restriction $\left.S_{\bar{x}} g S_{\bar{y}}^{*}\right|_{V}$ is trivial. This implies that $\bar{x}=\bar{y}$ and $g$ acts trivially on the open set $U \subset X^{\omega}$. We may assume without loss of generality that $U=\bar{z} X^{\omega}$ with $\bar{z} \in X^{k}$ and $k \in \mathbb{N}$. Since $g$ acts trivially on $U$, we have $g(\bar{z})=\bar{z}$ and $\left.g\right|_{\bar{z}}$ acts trivially on $X^{\omega}$. Furthermore, $\eta \in U$. Hence $\eta=\bar{z} \xi$ for some element $\xi \in X^{\omega}$. This implies that

$$
\alpha_{n, m}^{k}([\bar{x}, g, \bar{y}, \eta])=\left[\bar{x} g(\bar{z}),\left.g\right|_{\bar{z}}, \bar{x} \bar{z}, \xi\right] .
$$

If $\left.g\right|_{\bar{z}}=1$ then

$$
\alpha_{n, m}^{k}([\bar{x}, g, \bar{y}, \eta])=[\bar{x} \bar{z}, 1, \bar{x} \bar{z}, \xi],
$$

and $[\bar{x}, g, \bar{y}, \eta]$ is a unit in the groupoid $\mathcal{H}$. So $\psi$ is injective. Furthermore, for $\mathcal{H}$ to be effective, we have to ask for the action of $G$ on $X^{\omega}$ to be faithful. This is true since the action of $G$ on $X^{*}$ is faithful. The map $\psi$ is open and continuous since $\mathcal{H}_{n}$ is endowed with the inductive limit topology for all $n \in \mathbb{Z}$. 
3 Actions of Ore monoids 


\section{Colimit equivalent diagrams}

An interesting question when studying $\mathrm{C}^{*}$-dynamics is whether two (generalised) dynamical systems have isomorphic crossed products. For singly generated dynamical systems we may achieve that two dynamical systems give isomorphic crossed products by asking for an isomorphism between the objects being acted on; and this isomorphism should intertwine the actions in the obvious sense. We may also ask for two arrows with opposite directions between the objects being acted on such that their compositions give the generators of the dynamical systems we started with. This has been called strong shift-equivalence (see [32]). The description of crossed products of generalised actions in Corr as colimits of diagrams allows us, for instance, to realise that a strong shift equivalence is itself a diagram containing both actions as subdiagrams, so that the colimit of this diagram is isomorphic to the colimits of both subdiagrams.

We prove in this chapter that under certain assumptions on a diagram, we may restrict to any cofinal object and obtain an Ore monoid action with the same colimit as the original diagram. This is done in the abstract setting of a general bicategory. We verify in the last section that our notion of colimit equivalence generalises that of shift-equivalence for graphs and $\mathrm{C}^{*}$-correspondences when restricting to the bicategories $\mathfrak{C o r r}$ and $\mathfrak{G} r$.

\subsection{Colimit equivalent diagrams in general bicategories}

\section{Filtered categories}

In the following we relax the notion of filtered categories.

Definition 4.1. A nonempty category $\mathcal{C}$ is called weakly filtered if

(1) for any two objects $x, y \in \mathcal{C}_{0}$, there is an object $z \in \mathcal{C}_{0}$ and arrows $g \in \mathcal{C}(x, z)$ and $h \in \mathcal{C}(y, z)$;

(2) for any two parallel arrows $g, h \in \mathcal{C}\left(y_{1}, y_{2}\right)$, there is an object $y_{3} \in \mathcal{C}_{0}$ and arrows $k_{1}, k_{2} \in \mathcal{C}\left(y_{2}, y_{3}\right)$ such that $k_{1} g=k_{2} h$.

If we demand in (2) that $k_{1}=k_{2}$, then $\mathcal{C}$ is a filtered category in the notation of $[27, \S \mathrm{IX} .1]$.

From (1) and (2), it follows that 
(3) for all $g_{1}, g_{2} \in \mathcal{C}$ with $s\left(g_{1}\right)=s\left(g_{2}\right)$, there are $k_{1}, k_{2} \in \mathcal{C}$ such that $k_{1} g_{1}=k_{2} g_{2}$.

The category $\mathcal{C}$ is called 2 -directed if it is weakly filtered and

(4) if $k_{1} g=k_{2} g$ for $k_{1}, k_{2}, g \in \mathcal{C}$, then there is $h \in \mathcal{C}$ with $h k_{1}=h k_{2}$.

Notice that for monoids, the notions of Ore and 2-directed coincide.

Conditions (3)-(4) imply that we can construct a (discrete) groupoid from the category $\mathcal{C}$. This is done as follows. Let $X \rtimes P$ be the quotient of $\mathcal{C} \times{ }_{r} \mathcal{C}=$ $\left\{(g, h) \in \mathcal{C}_{1} \times \mathcal{C}_{1} \mid r(g)=r(h)\right\}$ by the equivalence relation $\left(g_{1}, h_{1}\right) \sim\left(g_{2}, h_{2}\right)$ if there are $k_{1}, k_{2} \in \mathcal{C}$ such that $\left(k_{1} g_{1}, k_{1} h_{1}\right)=\left(k_{2} g_{2}, k_{2} h_{2}\right)$. The object set of $X \rtimes P$ is identified with $\mathcal{C}_{0}$ by the map $x \mapsto\left[\left(\mathrm{id}_{x}, \mathrm{id}_{x}\right)\right]$. The source and range maps are given by $r(g, h):=s(g)$ and $s(g, h):=s(h)$ for all $(g, h) \in \mathcal{G}$. The multiplication is given by $\left(g_{1}, h_{1}\right)\left(g_{2}, h_{2}\right):=\left(k_{1} g_{1}, k_{2} h_{2}\right)$, where $k_{1}, k_{2} \in \mathcal{C}$ are such that $k_{1} h_{1}=k_{2} g_{2}$. Conditions (3) and (4) imply that the multiplication does not depend on the choice of $k_{1}, k_{2}$ and that it is well defined. The inverse of an element $[(g, h)] \in \mathcal{G}$ is given by $[(g, h)]^{-1}:=[(h, g)]$. Define $i_{\mathcal{C}}: \mathcal{C} \rightarrow \mathcal{G}$ by $i_{\mathcal{C}}(g):=\left[\left(\operatorname{id}_{r(g)}, g\right)\right]$. Then $i_{\mathcal{C}}$ is a faithful functor from $\mathcal{C}$ to $\mathcal{G}$.

Definition 4.2. Let $\mathcal{C}$ be a category and let $\mathcal{C}^{1}$ be a full subcategory of $\mathcal{C}$. We say that $\mathcal{C}^{1}$ is cofinal if for any object $y \in \mathcal{C}_{0}$, there is an object $x \in \mathcal{C}_{0}^{1}$ and an arrow $g \in \mathcal{C}(y, x)$. We say that an object $x$ is cofinal if the subcategory $\mathcal{C}(x, x)$ is cofinal.

Lemma 4.3. A cofinal subcategory $\mathcal{C}^{1}$ of a 2-directed category $\mathcal{C}$ is also 2-directed.

Proof. Let $\mathcal{C}^{1}$ be a cofinal subcategory of a 2 -directed category $\mathcal{C}$. We will show that conditions (1), (2) and (4) hold in $\mathcal{C}^{1}$. Condition (1) follows because every cofinal subcategory is full. Let $g_{1}, g_{2} \in \mathcal{C}^{1}$ with $s\left(g_{1}\right)=s\left(g_{2}\right)$. Then there are $k_{1}, k_{2} \in \mathcal{C}$ such that $k_{1} g_{1}=k_{2} g_{2}$. Since $\mathcal{C}^{1}$ is cofinal there is an object $z \in \mathcal{C}_{0}^{1}$ and an arrow $l: r\left(k_{2}\right) \rightarrow z$. The arrows $l k_{1}, l k_{2} \in \mathcal{C}^{1}$ satisfy $l k_{1} g_{1}=l k_{2} g_{2}$, and condition (2) follows. Condition (4) follows similarly.

Lemma 4.4. Let $\mathcal{C}$ be a 2-directed category and let $x$ be a cofinal object in $\mathcal{C}$. Then for every triple $(g, h, k)$ with $r(g)=r(k)=x$ and $h \in \mathcal{C}(s(k), s(g))$ there are $\xi_{1}, \xi_{2} \in \mathcal{C}(r(g), r(g))$ with $\xi_{1} g h=\xi_{2} k$.

Proof. Let $(g, h, k)$ be as in the assumption. Let $y_{1}=s(g)$ and $y_{2}=s(k)$.

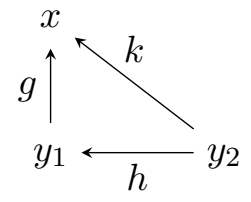


By our assumption that $\mathcal{C}$ is 2-directed, there is an object $y \in \mathcal{C}_{0}$, and there are elements $\eta_{1}, \eta_{2} \in \mathcal{C}(x, y)$ with $\eta_{1} g h=\eta_{2} k$. There is also an arrow $t \in \mathcal{C}(y, x)$ since $x$ is cofinal. Let $\xi_{1}:=t \eta_{1}$ and $\xi_{2}:=t \eta_{2}$. It follows that $\xi_{1} g h=\xi_{2} k$ with $\xi_{1}, \xi_{2} \in \mathcal{C}(x, x)$.

Let $\mathcal{G}, \mathcal{G}^{1}$ denote the groupoid completions of $\mathcal{C}, \mathcal{C}^{1}$ respectively. Then $\mathcal{G}^{1}$ is the restriction of $\mathcal{G}$ to the object set $\mathcal{G}_{0}^{1}$ if $\mathcal{C}^{1}$ is a cofinal subcategory of $\mathcal{C}$. In particular, if $x$ is an object in a 2-directed category $\mathcal{C}$ such that $\mathcal{C}(x, x)$ is cofinal, then the group completion of the monoid $\mathcal{C}(x, x)$ is the isotropy group of $x$ in the groupoid completion of $\mathcal{C}$.

Example 4.5. Let $\mathcal{C}_{s}$ be the path category of the directed graph

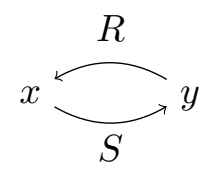

The arrows in $\mathcal{C}_{s}$ are composites $(S R)^{n},(R S)^{n}, R(S R)^{n}, S(R S)^{n}$ for $n \geq 0$, where $(S R)^{0}$ and $(R S)^{0}$ are interpreted as the identity arrows on $x$ and $y$, respectively. We claim that $\mathcal{C}_{s}$ is 2-directed. Condition (1) in Definition 4.1 is satisfied. To show that (2) holds, let $g$ and $h$ be two parallel arrows in $\mathcal{C}_{s}$. We have four cases. If $g, h: x \rightarrow x$, then they are necessarily of the form $g=(S R)^{n}$ and $(S R)^{m}$ for $n, m \in \mathbb{N}$. We assume without loss of generality that $n \geq m$. We have $(S R)^{n-m}(S R)^{m}=(S R)^{n}$ and we may choose $k_{1}=(S R)^{n-m}$ and $k_{2}=$ id. The other three cases can be treated similarly. It follows that $\mathcal{C}_{s}$ is weakly filtered. In addition, Condition (4) holds, even more, $\mathcal{C}_{s}$ is path cancellative. This implies that $\mathcal{C}_{s}$ is 2-directed. Notice that it is not a filtered category. It is routine to check that the full subcategories $\mathcal{C}_{s}(x, x)$ and $\mathcal{C}_{s}(y, y)$ are cofinal in $\mathcal{C}_{s}$.

More generally, for $n \in \mathbb{N}$, let $\mathcal{D}_{s}^{n}$ be the path category of
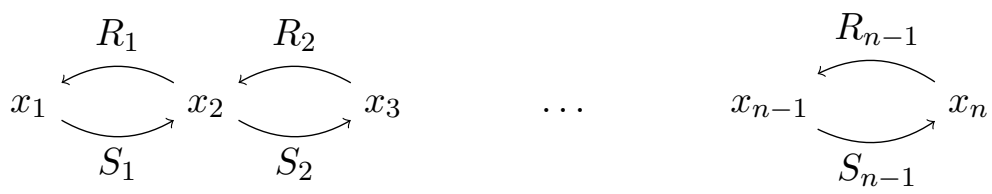

And let $\mathcal{C}_{s}^{n}$ be the quotient of $\mathcal{D}_{s}^{n}$ generated by the relations $R_{i-1} S_{i-1} \sim S_{i} R_{i}$ for $i=2, \ldots, n-1$. In this case, the full subcategories $\mathcal{C}_{s}^{n}\left(x_{1}, x_{1}\right)$ and $\mathcal{C}_{s}^{n}\left(x_{n}, x_{n}\right)$ are cofinal in $\mathcal{C}_{s}^{n}$. We prove next that $\mathcal{C}_{s}^{n}$ is 2-directed. Let $1 \leq i \leq l \leq j \leq n$ and let $g=\left(R_{j} S_{j}\right)^{k} S_{j-1} \cdots S_{l-1} \cdot\left(S_{l} R_{l}\right)^{a} \cdot S_{l} \cdots S_{i}$ be an arrow from $x_{i}$ to $x_{j}$ with $a$, $k \in \mathbb{N}$. Then using the identifications $R_{m-1} S_{m-1} \sim S_{m} R_{m}$ for $m=2, \ldots, n-1$ 
we get

$$
\begin{aligned}
g & =\left(R_{j} S_{j}\right)^{k} S_{j-1} \cdots S_{l-1} \cdot\left(S_{l} R_{l}\right)^{a} \cdot S_{l} \cdots S_{i} \\
& =\left(R_{j} S_{j}\right)^{k} S_{j-1} \cdots S_{l-1} \cdot\left(R_{l-1} S_{l-1}\right)^{a} \cdot S_{l} \cdots S_{i} \\
& =\left(R_{j} S_{j}\right)^{k} S_{j-1} \cdots S_{l-2} \cdot\left(S_{l-1} R_{l-1}\right)^{a} S_{l-1} \cdot S_{l} \cdots S_{i} \\
& =\left(R_{j} S_{j}\right)^{k} S_{j-1} \cdots S_{l-2} \cdot\left(R_{l-2} S_{l-1}\right)^{a} S_{l-1} \cdots S_{i} \\
& =\left(R_{j} S_{j}\right)^{k+a} S_{j-1} \cdots S_{i} .
\end{aligned}
$$

It follows that any arrow from $x_{i}$ to $x_{j}$ for $1 \leq i \leq j \leq n$ is of the form $\left(R_{j} S_{j}\right)^{k} S_{j-1} \cdots S_{i}$ for $k \in \mathbb{N}$. Let $k_{1}, k_{2} \in \mathbb{N}$ and let $g=\left(R_{j} S_{j}\right)^{k_{1}} S_{j-1} \cdots S_{i}$, $h=\left(R_{j} S_{j}\right)^{k_{2}} S_{j-1} \cdots S_{i}$ be two such arrows. We may assume without loss of generality that $k_{1} \geq k_{2}$. Then we have $\left(R_{j} S_{j}\right)^{k_{1}-k_{2}} h=g$ and therefore Condition (2) in Definition 4.1 is satisfied. The other conditions in Definition 4.1 follow as in the case of $n=2$.

Definition 4.6. Let $\mathcal{M}$ be a bicategory with all 2-arrows being invertible. We call two diagrams $\mathcal{D}_{1}$ and $\mathcal{D}_{2}$ colimit equivalent if they have the same colimit.

Theorem 4.7. Let $\mathcal{M}$ be a bicategory with all 2-arrows being invertible. Let $\mathcal{C}$ be a 2-directed category and $x \in \mathcal{C}_{0}$ a cofinal object. Let $F: \mathcal{C} \rightarrow \mathcal{M}$ be a functor, and let $F_{x}$ be its restriction to the monoid $\mathcal{C}(x, x)$. Then $F$ and $F_{x}$ have equivalent colimits.

Proof. We will make an extensive use of the coherence conditions (2.3) and (2.8) in this proof. To avoid tedious repetition of these arguments, we will omit parts of the calculations to make the proof clearer.

Let $D \in \mathcal{M}^{0}$ be an object. We want an equivalence of groupoids

$$
R_{x}: \mathcal{M}^{\mathcal{C}(x, x)}\left(F_{x}, \text { const }_{D}\right) \stackrel{\cong}{\rightrightarrows} \mathcal{M}^{\mathcal{C}}\left(F, \text { const }_{D}\right) .
$$

This implies the equivalence of the colimits for both diagrams. Recall that colimits are only well-defined up to equivalence in $\mathcal{M}$.

Since $\mathcal{C}(x, x)$ is a full subcategory of $\mathcal{C}$ we have an obvious map

$$
\Psi_{x}: \mathcal{M}^{\mathcal{C}}\left(F, \text { const }_{D}\right) \rightarrow \mathcal{M}^{\mathcal{C}(x, x)}\left(F_{x}, \text { const }_{D}\right) .
$$

This map sends an object $(\mathcal{G}, V) \in \mathcal{M}^{\mathcal{C}}\left(F, \text { const }_{D}\right)_{0}$ (a transformation) to its restriction $\left(\mathcal{G}_{x}, V_{x}\right)$ to the monoid $\mathcal{C}(x, x)$. The image under the map $\Psi_{x}$ of a modification $W:\left(\mathcal{G}^{1}, V^{1}\right) \rightarrow\left(\mathcal{G}^{2}, V^{2}\right)$ is the restricted modification $W_{x}:\left(\mathcal{G}_{x}^{1}, V_{x}^{1}\right) \rightarrow\left(\mathcal{G}_{x}^{2}, V_{x}^{2}\right)$. We need an inverse $R_{x}$ of $\Psi_{x}$. Let $\left(T_{x}, U_{g}\right) \in \mathcal{M}^{\mathcal{C}(x, x)}\left(F_{x} \text {, } \text { const }_{D}\right)_{0}$ be a transformation (see Definition 2.5). We want to extend this transformation to a transformation from $F$ to const $_{D}$. 
First, let $y \in \mathcal{C}^{0}$. The object $x$ is cofinal. So we can choose an arrow $h_{y}: y \rightarrow x$. We define

$$
T_{y}:=T_{x} \circ F\left(h_{y}\right) .
$$

Secondly, let $g: y_{2} \rightarrow y_{1}$ be an arrow in $\mathcal{C}$. Since $\mathcal{C}$ is 2-directed and $x$ is cofinal, Lemma 4.4 gives $\xi_{1}, \xi_{2} \in \mathcal{C}(x, x)$ such that

$$
\xi_{1} h_{y_{1}} g=\xi_{2} h_{y_{2}} .
$$

Let $\phi_{g}^{\xi_{1}, \xi_{2}}: F\left(\xi_{2}\right) F\left(h_{y_{2}}\right) \rightarrow F\left(\xi_{1}\right) F\left(h_{y_{1}}\right) F(g)$ be the 2-arrow given by

$$
\begin{aligned}
\phi_{g}^{\xi_{1}, \xi_{2}} & :=\left(\operatorname{id}_{F_{\xi_{1}}} * u_{h_{y_{1}}, g}^{-1}\right) * u_{\xi_{1}, h_{y_{1}} g}^{-1} * u_{\xi_{2}, h_{y_{2}}} \\
& =\left(u_{\xi_{1}, h_{y_{1}}}^{-1} * \operatorname{id}_{F(g)}\right) * u_{\xi_{1} h_{y_{1}}, g}^{-1} * u_{\xi_{2}, h_{y_{2}}} .
\end{aligned}
$$

The last equality follows from the associativity condition (2.3). We define

$$
U_{g}^{\xi_{1}, \xi_{2}}:=\left(U_{\xi_{1}}^{-1} * \operatorname{id}_{F\left(h_{y_{1}}\right) \circ F(g)}\right)\left(\operatorname{id}_{T_{x}} * \phi_{g}^{\xi_{1}, \xi_{2}}\right) *\left(U_{\xi_{2}} * \operatorname{id}_{F\left(h_{y_{2}}\right)}\right) .
$$

This is a 2-arrows $U_{g}^{\xi_{1}, \xi_{2}}$ from $T_{y_{2}}=T_{x} \circ F\left(h_{y_{2}}\right)$ to $T_{y_{1}} \circ F(g)=T_{x} \circ F\left(h_{y_{1}}\right) \circ F(g)$.

Lemma 4.8. The 2-arrow $U_{g}^{\xi_{1}, \xi_{2}}$ does not depend on the choice of $\xi_{1}$ and $\xi_{2}$.

Proof. Let $g, \xi_{1}$ and $\xi_{2}$ as before. First we show that for all $k \in \mathcal{C}(x, x)$ we have

$$
U_{g}^{k \xi_{1}, k \xi_{2}}=U_{g}^{\xi_{1}, \xi_{2}}
$$

By repeated application of (2.3):

$$
\begin{aligned}
\phi_{g}^{k \xi_{1}, k \xi_{2}}\left(u_{k, \xi_{2}} * \operatorname{id}_{F\left(h_{y_{2}}\right)}\right) \\
=\left(u_{k \xi_{1}, h_{y_{1}}}^{-1} * \operatorname{id}_{F(g)}\right) u_{k \xi_{1} h_{y_{1}}, g}^{-1} \cdot u_{k \xi_{2}, h_{y_{2}}}\left(u_{k, \xi_{2}} * \operatorname{id}_{F\left(h_{y_{2}}\right)}\right) \\
=\left(u_{k \xi_{1}, h_{y_{1}}}^{-1} \operatorname{id}_{F(g)}\right) u_{k \xi_{1} h_{y_{1}}, g}^{-1} \cdot u_{k, \xi_{2} h_{y_{2}}}\left(\operatorname{id}_{F(k)} * u_{\xi_{2}, h_{y_{2}}}\right) \\
=\left(u_{k \xi_{1}, h_{y_{1}}}^{-1} * \operatorname{id}_{F(g)}\right) u_{k \xi_{1} h_{y_{1}}, g}^{-1} \cdot u_{k, \xi_{1} h_{y_{1}} g}\left(\operatorname{id}_{F(k)} * u_{\xi_{2}, h_{y_{2}}}\right) \\
=\left(u_{k \xi_{1}, h_{y_{1}}}^{-1} * \operatorname{id}_{F(g)}\right)\left(u_{k, \xi_{1} h_{y_{1}}} * \operatorname{id}_{F(g)}\right)\left(\operatorname{id}_{F(k)} * u_{\xi_{1} h_{y_{1}}, g}^{-1}\right)\left(\operatorname{id}_{F(k)} * u_{\xi_{2}, h_{y_{2}}}\right) \\
=\left(u_{k, \xi_{1}} * \operatorname{id}_{F\left(h_{y_{1}}\right) \circ F(g)}\right)\left(\operatorname{id}_{F_{k}} * \phi_{g}^{\xi_{1}, \xi_{2}}\right) .
\end{aligned}
$$

Hence

$$
\phi_{g}^{k \xi_{1}, k \xi_{2}}=\left(u_{k, \xi_{1}} * \operatorname{id}_{F\left(h_{y_{1}}\right) \circ F(g)}\right)\left(\operatorname{id}_{F_{k}} * \phi_{g}^{\xi_{1}, \xi_{2}}\right)\left(u_{k, \xi_{2}}^{-1} * \operatorname{id}_{F\left(h_{y_{2}}\right)}\right)
$$


Therefore,

$$
\begin{aligned}
& U_{g}^{k \xi_{1}, k \xi_{2}}=\left(U_{k \xi_{1}}^{-1} * \operatorname{id}_{F\left(h_{y_{1}}\right) \circ F(g)}\right)\left(\operatorname{id}_{T_{x}} * \phi_{g}^{k \xi_{1}, k \xi_{2}}\right) *\left(U_{k \xi_{2}} * \operatorname{id}_{F\left(h_{y_{2}}\right)}\right) \\
&=\left(U_{\xi_{1}}^{-1} * \operatorname{id}_{F\left(h_{y_{1}}\right) \circ F(g)}\right)\left(U_{k}^{-1} * \operatorname{id}_{F\left(\xi_{1}\right)}\right) *\left(\operatorname{id}_{F(k)} * \phi_{g}^{\xi_{1}}, \xi_{2}\right) * \\
&\left(\left(\left(U_{k} * \operatorname{id}_{F\left(\xi_{2}\right)}\right) * U_{\xi_{2}}\right) * \operatorname{id}_{F_{h_{y_{2}}}}\right)=U_{g}^{\xi_{1}, \xi_{2}}
\end{aligned}
$$

Here we used the identity $U_{k \xi_{2}}=\left(\operatorname{id}_{T_{x}} * u_{k, \xi_{2}}\right)\left(U_{k} * \mathrm{id}_{F\left(\xi_{2}\right)}\right) * U_{\xi_{2}}$ and a similar identity for $U_{k \xi_{1}}^{-1}$, as $\left(T_{x}, U_{g}\right) \in \mathcal{M}^{\mathcal{C}(x, x)}\left(F_{x}\right.$, const $\left._{D}\right)$ is a transformation and hence satisfies (2.8).

Now let $\left(\eta_{1}, \eta_{2}\right) \in \mathcal{C}(x, x)$ be another pair satisfying $\eta_{1} h_{y_{1}} g=\eta_{2} h_{y_{2}}$. There are $k_{1}, k_{2} \in \mathcal{C}(x, x)$ such that $k_{1} \xi_{1}=k_{2} \eta_{1}$. Hence $k_{1} \xi_{2} h_{y_{2}}=k_{2} \eta_{2} h_{y_{2}}$. Since $\mathcal{C}$ is 2-directed (Definition 4.1), there is an element $z \in \mathcal{C}(x, x)$ such that $z k_{1} \xi_{2}=z k_{2} \eta_{2}$. Therefore, we have

$$
U_{g}^{\xi_{1}, \xi_{2}}=U_{g}^{z k_{1} \xi_{1}, z k_{1} \xi_{2}}=U_{g}^{z k_{2} \eta_{1}, z k_{2} \eta_{2}}=U_{g}^{\eta_{1}, \eta_{2}}
$$

So, the 2-arrow $U_{g}^{\xi_{1}, \xi_{2}}$ does not depend on the choice of the pair $\left(\xi_{1}, \xi_{2}\right)$.

Let $U_{g}:=U_{g}^{\xi_{1}, \xi_{2}}$. Next we verify the coherence condition (2.8). Let $g: y_{2} \rightarrow y_{1}$ and $h: y_{3} \rightarrow y_{2}$ be two composable arrows in $\mathcal{C}$. Then there are $\xi_{1}, \xi_{2}, \xi_{2}^{\prime}$ and $\xi_{3} \in \mathcal{C}(x, x)$ with $\xi_{2} h_{y_{2}} h=\xi_{3} h_{y_{3}}$ and $\xi_{1} h_{y_{1}} g=\xi_{2}^{\prime} h_{y_{2}}$. Since $\mathcal{C}(x, x)$ is Ore, there are $\eta_{1}, \eta_{2} \in \mathcal{C}(x, x)$ with $\eta_{1} \xi_{2}^{\prime}=\eta_{2} \xi_{2}$. Then

$$
\eta_{1} \xi_{1} h_{y_{1}} g h=\eta_{1} \xi_{2}^{\prime} h_{y_{2}} h=\eta_{2} \xi_{2} h_{y_{2}} h=\eta_{2} \xi_{3} h_{y_{3}} .
$$

The elements $\eta \xi_{1}, \eta_{1} \xi_{2}^{\prime}=\eta_{2} \xi_{2}$ and $\eta_{2} \xi_{3}$ satisfy $\eta_{1} \xi_{1} h_{y_{1}} g=\eta_{1} \xi_{2}^{\prime} h_{y_{2}}, \eta_{1} \xi_{2}^{\prime} h_{y_{2}} h=$ $\eta_{2} \xi_{3} h_{y_{3}}$ and $\eta_{1} \xi_{1} h_{y_{1}} g h=\eta_{2} \xi_{3} h_{y_{3}}$. Therefore, without loss of generality, we may choose $\xi_{2}=\xi_{2}^{\prime}$; and we get

$$
\xi_{1} h_{y_{1}} g h=\xi_{2} h_{y_{2}} h=\xi_{3} h_{y_{3}} .
$$

Similar computations as in the proof of (4.3) show that

$$
\left(\operatorname{id}_{F\left(\xi_{1}\right) F\left(h_{y_{1}}\right)} * u_{g, h}^{-1}\right) \phi_{g h}^{\xi_{1}, \xi_{3}}=\left(\phi_{g}^{\xi_{1}, \xi_{2}} * \operatorname{id}_{F_{h}}\right) \phi_{h}^{\xi_{2}, \xi_{3}}
$$

This equation will be relevant in the following. 
To check (2.8), we start with the following commuting diagram:

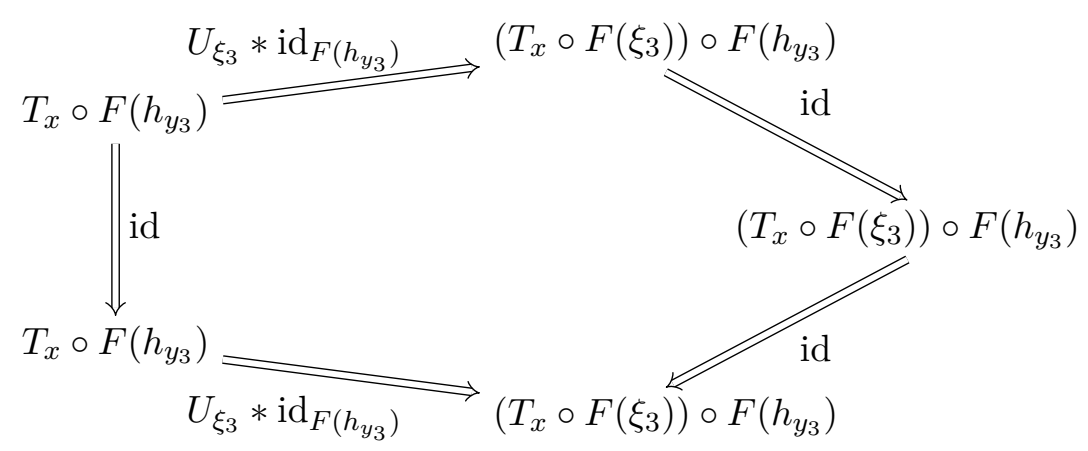

Let

$$
\begin{aligned}
& K_{1}:=\left(U_{\xi_{2}}^{-1} * \operatorname{id}_{F\left(h_{y_{2}}\right) \circ F(h)}\right)\left(\operatorname{id}_{T_{x}} * \phi_{h}^{\xi_{2}, \xi_{3}}\right), \\
& K_{2}:=\left(U_{\xi_{1}}^{-1} * \operatorname{id}_{F\left(h_{y_{1}}\right) \circ F(g) \circ F(h)}\right)\left(\operatorname{id}_{T_{x}} * \phi_{g}^{\xi_{1}, \xi_{2}} * \operatorname{id}_{F(h)}\right)\left(\operatorname{id}_{T_{x}} * \phi_{h}^{\xi_{2}, \xi_{3}}\right), \\
& K_{3}:=\left(U_{\xi_{1}}^{-1} * \operatorname{id}_{F\left(h_{y_{1}}\right) \circ F(g h)}\right)\left(\operatorname{id}_{T_{x}} * \phi_{g h}^{\xi_{1}, \xi_{3}}\right) .
\end{aligned}
$$

Then the diagram

$$
\begin{aligned}
& K_{1} *\left(U_{\xi_{3}} * \operatorname{id}_{F\left(h_{y_{3}}\right)}\right) \\
& T_{x} \circ F\left(h_{y_{3}}\right) \stackrel{T_{x} \circ F\left(h_{y_{2}}\right) \circ F(h)}{K_{2} * \mathrm{id} * K_{1}^{-1}} \\
& \qquad \mathrm{id} \\
& T_{x} \circ F\left(h_{y_{3}}\right) \underset{T_{x} \circ F\left(h_{y_{1}}\right) \circ F(g) \circ F(h)}{K_{3} *\left(U_{\xi_{3}} * \mathrm{id}_{F\left(h_{y_{3}}\right)}\right)} T_{x} \circ F\left(h_{y_{1}}\right) \circ F_{g h} * \mathrm{id} * K_{2}^{-1}
\end{aligned}
$$

also commutes. Computing the expressions in the diagram gives

$$
\begin{aligned}
K_{1} *\left(U_{\xi_{3}} * \mathrm{id}_{F\left(h_{y_{3}}\right)}\right) & =U_{h}, \\
K_{2} * \mathrm{id} * K_{1}^{-1} & =U_{g} * \operatorname{id}_{F(h)}, \\
K_{3} * \mathrm{id} * K_{2}^{-1} & =\operatorname{id}_{\left(T_{x} \circ F\left(h_{y_{1}}\right)\right)} * u_{g, h}, \\
K_{3} *\left(U_{\xi_{3}} * \operatorname{id}_{F\left(h_{y_{3}}\right)}\right) & =U_{g h} .
\end{aligned}
$$

So the following diagram commutes:

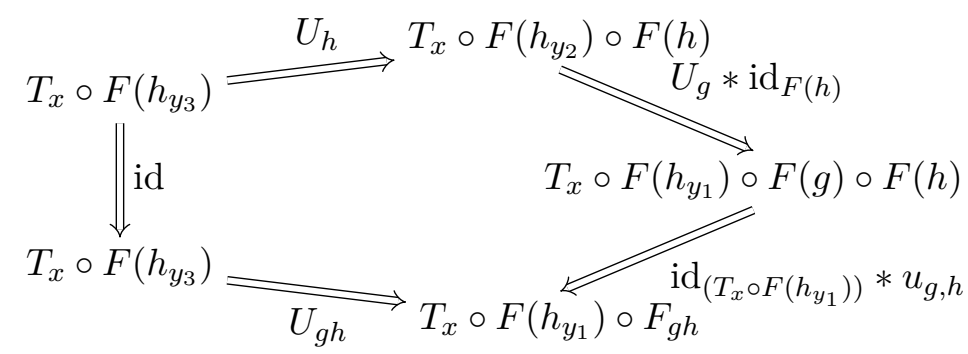


Substituting $T_{y_{i}}=T_{x} \circ F_{h_{y_{i}}}$ for $i=1,2,3$, in the last diagram, we get the coherence condition in $(2.8)$. So $(T, U)$ is a transformation from $\mathcal{C}$ to const ${ }_{D}$. Let $\left(T_{x}^{1}, U_{\xi}^{1}\right)$ and $\left(T_{x}^{1}, U_{\xi}^{1}\right)$ be transformations in $\mathcal{M}^{\mathcal{C}(x, x)}\left(F_{x}\right.$, const $\left._{D}\right)$, and let $W_{x}: T_{x}^{1} \rightarrow T_{x}^{2}$ be a modification between $\left(T_{x}^{1}, U_{\xi}^{1}\right)$ and $\left(T_{x}^{1}, U_{\xi}^{1}\right)$. As we have seen before, we can extend $\left(T_{x}^{i}, U_{\xi}^{i}\right)$ to a transformation $\left(T^{i}, U_{g}^{i}\right) \in \mathcal{M}^{\mathcal{C}}\left(F\right.$, const $\left._{D}\right)$ for $i=1,2$. Next we show that we can extend the modification $W_{x}$ to a modification $W$ from $\left(T^{1}, U_{g}^{1}\right)$ to $\left(T^{2}, U_{g}^{2}\right)$. For all $y \in \mathcal{C}_{0}$, define $W_{y}: T_{y}^{1} \rightarrow T_{y}^{2}$ by

$$
W_{y}:=W_{x} * \operatorname{id}_{F\left(h_{y}\right)} .
$$

We verify that $W$ satisfies the coherence condition (2.10). Let $g: y_{1} \rightarrow y_{2}$ be an arrow in $\mathcal{C}$ and let $\xi_{1}, \xi_{2}$ be such that $\xi_{1} h_{y_{1}} g=\xi_{2} h_{y_{2}}$. The coherence condition (2.10) is satisfied for the monoid $\mathcal{C}(x, x)$. Therefore, the following diagram commutes:

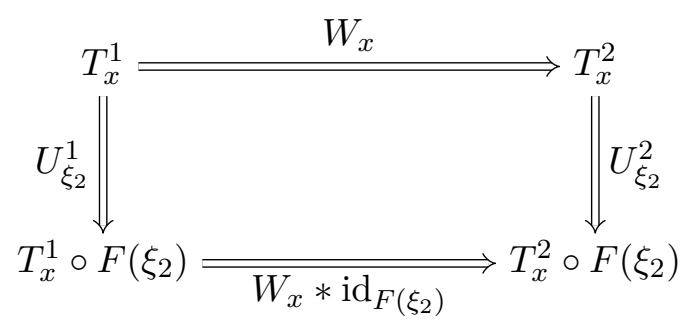

Hence

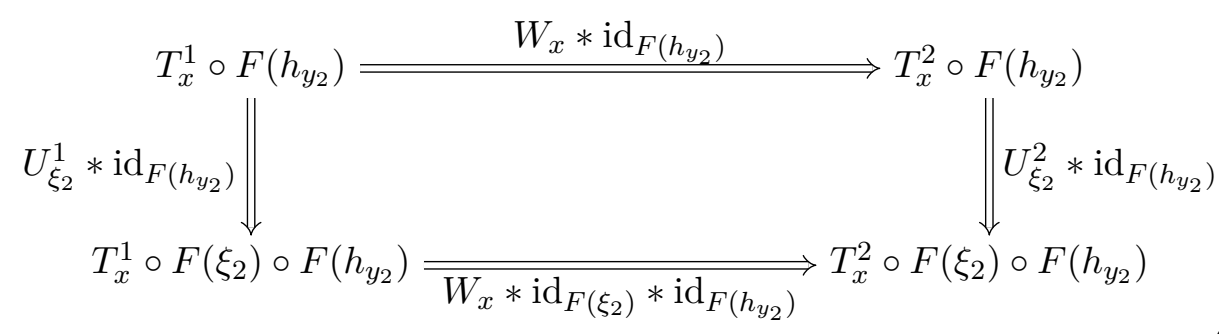

also commutes. Next, notice that

$$
\begin{aligned}
& \left(U_{\xi_{1}}^{2-1} * \operatorname{id}_{F\left(h_{y_{1}}\right) \circ F(g)}\right)\left(\operatorname{id}_{T_{x}} * \phi_{g}^{\xi_{1}, \xi_{2}}\right)\left(W_{x} * \operatorname{id}_{F\left(\xi_{2}\right) \circ F\left(h_{y_{2}}\right)}\right) \\
& \left(\operatorname{id}_{T_{x}} * \phi_{g}^{\xi_{1}, \xi_{2}}-1\right)\left(U_{\xi_{1}}^{1} * \operatorname{id}_{F\left(h_{y_{1}}\right) \circ F(g)}\right)=W_{y_{1}} * \operatorname{id}_{F(g)} .
\end{aligned}
$$

The last equality holds since $W_{x}=U_{\xi_{1}}^{2-1}\left(W_{x} * \mathrm{id}_{F\left(\xi_{1}\right)}\right) U_{\xi_{1}}^{1}$, see (4.8). Hence multiplying $U_{\xi_{2}}^{1} * \operatorname{id}_{F\left(h_{y_{2}}\right)}$ in (4.9) with $\left(U_{\xi_{1}}^{1}{ }^{-1} * \operatorname{id}_{F\left(h_{y_{1}}\right) \circ F(g)}\right)\left(\operatorname{id}_{T_{x}} * \phi_{g}^{\xi_{1}, \xi_{2}}\right)$ and multiplying $U_{\xi_{2}}^{2} * \operatorname{id}_{F\left(h_{y_{2}}\right)}$ with $\left(U_{\xi_{1}}^{2-1} * \operatorname{id}_{F\left(h_{y_{1}}\right) \circ F(g)}\right)\left(\operatorname{id}_{T_{x}} * \phi_{g}^{\xi_{1}, \xi_{2}}\right)$, we get that the 
diagram

$$
\begin{aligned}
& T_{y_{2}}^{1} \Longrightarrow W_{y_{2}}^{2} \\
& U_{g}^{1} \Downarrow \quad \Downarrow U_{g}^{2} \\
& T_{y_{1}}^{1} \circ F(g) \Longrightarrow T_{y_{1}}^{2} \circ F(g)
\end{aligned}
$$

commutes. Therefore, $W$ is a modification. Now let $W^{1}$ be another modification from $\left(T^{1}, U_{g}^{1}\right)$ to $\left(T^{2}, U_{g}^{2}\right)$ with $W_{x}=W_{x}^{1}$. Let $y \in \mathcal{C}^{0}$. Then the following diagram commutes:

$$
\begin{array}{cc}
T_{y}^{1} & W_{y}^{1} \\
U_{h_{y}}^{1}=\mathrm{id} \Downarrow & T_{y}^{2} \\
T_{x}^{1} \circ F\left(h_{y}\right) \stackrel{\Downarrow U_{h_{y}}^{2}=\mathrm{id}}{\rightleftharpoons W_{x}^{1} * \operatorname{id}_{F\left(h_{y}\right)}} T_{x}^{2} \circ F\left(h_{y}\right)
\end{array}
$$

Thus $W_{y}=W_{x} * \operatorname{id}_{F\left(h_{y}\right)}$. So the modification $W^{1}$ is uniquely determined by $W_{x}^{1}=W_{x}$.

Corollary 4.9. Let $\mathcal{C}$ be a 2-directed category and $\mathcal{C}_{1}$ be a connected cofinal subcategory of $\mathcal{C}$. Let $F: \mathcal{C} \rightarrow \mathcal{M}$ be a functor and let $F_{1}$ be its restriction to $\mathcal{C}_{1}$. Then $F$ and $F_{1}$ have equivalent colimits.

Let $\mathcal{C}$ be a 2-directed category and let $x, y \in \mathcal{C}_{0}$ be such that $\mathcal{C}(x, x), \mathcal{C}(y, y)$ are cofinal. Let $F: \mathcal{C} \rightarrow \mathcal{M}$ be a functor and let $F_{x}, F_{y}$ be its restrictions to the monoids $\mathcal{C}(x, x)$ and $\mathcal{C}(y, y)$, respectively. A direct consequence of Corollary 4.9 is that $F_{x}$ and $F_{y}$ have equivalent colimits.

\subsection{Applications}

We apply Theorem 4.7 in the bicategories $\mathfrak{G r}$ and $\mathfrak{C o r r}$

\subsubsection{Shift equivalence of $\mathrm{C}^{*}$-correspondences}

Muhly, Pask and Tomforde [32] introduced a notion of equivalence for correspondences which generalises "elementary strong shift equivalence" of graphs. This notion also has an elegant interpretation in terms of colimits, which then allows to extend it to diagrams of more general shape.

Definition 4.10. Let $A$ and $B$ be $\mathrm{C}^{*}$-algebras and let $\mathcal{E}_{1}: A \rightarrow A$ and $\mathcal{E}_{2}: B \rightarrow B$ be correspondences. An elementary strong shift equivalence between $\mathcal{E}_{1}$ and $\mathcal{E}_{2}$ consists of correspondences $\mathcal{E}_{R}: A \rightarrow B$ and $\mathcal{E}_{S}: B \rightarrow A$ with

$$
\mathcal{E}_{1} \cong \mathcal{E}_{R} \otimes_{A} \mathcal{E}_{S} \quad \text { and } \quad \mathcal{E}_{2} \cong \mathcal{E}_{S} \otimes_{B} \mathcal{E}_{R}
$$


Let strong shift equivalence be the equivalence relation generated by elementary strong shift equivalence. Thus a strong shift equivalence between $\mathcal{E}_{1}$ and $\mathcal{E}_{2}$ consists of

- $\mathrm{C}^{*}$-algebras $A_{1} \ldots A_{n}$ for $n \in \mathbb{N}$ such that $A_{1}=A$ and $A_{n}=B$;

- correspondences $\mathcal{E}_{R_{i}}: A_{i} \rightarrow A_{i+1}$ and $\mathcal{E}_{S_{i}}: A_{i+1} \rightarrow A_{i}$ for $i=1 \ldots n-1$ such that

(1) $\mathcal{E}_{1} \cong \mathcal{E}_{R_{1}} \otimes_{A_{2}} \mathcal{E}_{S_{1}} \quad$ and $\quad \mathcal{E}_{2} \cong \mathcal{E}_{S_{n-1}} \otimes_{A_{n-1}} \mathcal{E}_{R_{n-1}}$,

(2) $\mathcal{E}_{S_{i-1}} \otimes_{A_{i}} \mathcal{E}_{R_{i-1}} \cong \mathcal{E}_{R_{i}} \otimes_{A_{i+1}} \mathcal{E}_{S_{i}}$ for $i=2, \ldots, n-1$.

We recall the definition of the categories $\mathcal{C}_{s}$ and $\mathcal{C}_{s}^{n}$ from Example 4.5. An elementary strong shift equivalence between $\mathcal{E}_{1}$ and $\mathcal{E}_{2}$ defines a functor from $\mathcal{C}_{s}$

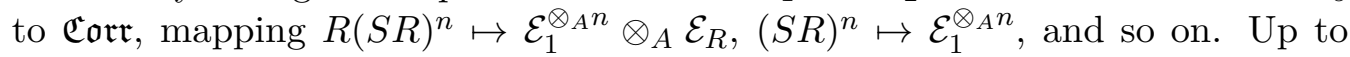
isomorphism, this functor is determined by $\mathcal{E}_{R}$ and $\mathcal{E}_{S}$ because $\mathcal{C}_{s}$ is generated freely by the arrows $R$ and $S$.

Let $\mathcal{E}_{R_{i}}: A_{i} \rightarrow A_{i+1}$ and $\mathcal{E}_{S_{i}}: A_{i+1} \rightarrow A_{i}$ for $i=1, \ldots, n-1$ be a strong shift equivalence between $\mathcal{E}_{1}$ and $\mathcal{E}_{2}$. As before, the assignments $S_{i} \mapsto \mathcal{E}_{S_{i}}$ and $R_{i} \mapsto \mathcal{E}_{R_{i}}$ for $i=1, \ldots, n-1$, determine a functor $\mathcal{S}^{\prime}: \mathcal{D}_{s}^{n} \rightarrow \mathfrak{C o r r}$. The isomorphisms in Condition (2) allow to descend this to a functor $\mathcal{S}: \mathcal{C}_{s}^{n} \rightarrow \mathfrak{C o r r}$. Theorem 4.7 implies that the colimit of the diagram $\mathcal{S}: \mathcal{C}_{s}^{n} \rightarrow \operatorname{Corr}$ in $\mathfrak{C o r r}$ is Morita-Rieffel equivalent to the colimit of either of the diagrams $\mathcal{S}_{x}: \mathbb{N} \rightarrow \mathfrak{C o r r}$ and $\mathcal{S}_{y}: \mathbb{N} \rightarrow \mathfrak{C o r r}$ obtained by restricting $\mathcal{S}$ to $\mathcal{C}_{s}^{n}(x, x)$ and $\mathcal{C}_{s}^{n}(y, y)$, respectively. We include a direct proof of this fact for $n=1$.

Proposition 4.11. Let $\mathcal{S}$ be the functor $\mathcal{C}_{s} \rightarrow \mathfrak{C o r r}_{\text {prop }}$ corresponding to the strong shift equivalence

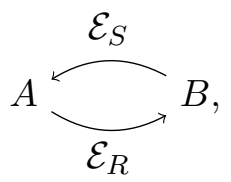

where $A$ and $B$ are $\mathrm{C}^{*}$-algebras and $\mathcal{E}_{R}$ and $\mathcal{E}_{S}$ are proper correspondences. Let $\mathcal{S}_{1}$ and $\mathcal{S}_{2}$ be the diagrams $(\mathbb{N},+) \rightarrow \mathfrak{C o r r}_{\text {prop }}$ generated by $\mathcal{E}_{R} \otimes_{B} \mathcal{E}_{S}: A \rightarrow A$ and $\mathcal{E}_{S} \otimes_{A}$ $\mathcal{E}_{R}: B \rightarrow B$, respectively. Then

$$
\operatorname{colim}(\mathcal{S}) \cong \operatorname{colim}\left(\mathcal{S}_{1}\right) \cong \operatorname{colim}\left(\mathcal{S}_{2}\right)
$$

in $\mathfrak{C o r r}$, that is, these colimits are Morita-Rieffel equivalent.

Proof. Let $D$ be a $\mathrm{C}^{*}$-algebra. A transformation from $\mathcal{S}$ to const $_{D}$ is a quadruple $\left(\mathcal{F}_{A}, \mathcal{F}_{B}, U_{S}, U_{R}\right)$, where

$$
\mathcal{F}_{A}: A \rightarrow D, \quad \mathcal{F}_{B}: B \rightarrow D
$$


are correspondences and

$$
U_{S}: \mathcal{E}_{S} \otimes_{A} \mathcal{F}_{A} \rightarrow \mathcal{F}_{B}, \quad U_{R}: \mathcal{E}_{R} \otimes_{B} \mathcal{F}_{B} \rightarrow \mathcal{F}_{A}
$$

are isomorphisms of correspondences. This induces isomorphisms

$$
U_{A}:\left(\mathcal{E}_{R} \otimes_{B} \mathcal{E}_{S}\right) \otimes_{A} \mathcal{F}_{A} \rightarrow \mathcal{F}_{A}, \quad U_{B}:\left(\mathcal{E}_{S} \otimes_{A} \mathcal{E}_{R}\right) \otimes_{B} \mathcal{F}_{B} \rightarrow \mathcal{F}_{B}
$$

Here we use implicitly that $\mathfrak{C o r r}$ is associative up to canonical isomorphism. Hence we get transformations $\left(\mathcal{F}_{A}, U_{A}\right)$ and $\left(\mathcal{F}_{B}, U_{B}\right)$ from $\mathcal{S}_{1}$ and $\mathcal{S}_{2}$ to const ${ }_{D}$, respectively. Conversely, let $\left(\mathcal{F}_{A}, U_{A}\right)$ be a transformation from $\mathcal{S}_{1}$ to const $_{D}$. Define

$$
\mathcal{F}_{B}:=\mathcal{E}_{S} \otimes_{A} \mathcal{F}_{A}, \quad U_{S}:=\operatorname{id}_{\mathcal{F}_{B}},
$$

and let $U_{R}: \mathcal{E}_{R} \otimes_{B} \mathcal{F}_{B} \rightarrow \mathcal{F}_{A}$ be the composite of $U_{A}:\left(\mathcal{E}_{R} \otimes_{B} \mathcal{E}_{S}\right) \otimes_{A} \mathcal{F}_{A} \rightarrow \mathcal{F}_{A}$ and the associator $\mathcal{E}_{R} \otimes_{B} \mathcal{F}_{B} \cong\left(\mathcal{E}_{R} \otimes_{B} \mathcal{E}_{S}\right) \otimes_{A} \mathcal{F}_{A}$. This gives a transformation $\left(\mathcal{F}_{A}, \mathcal{F}_{B}, U_{S}, U_{R}\right)$ from $\mathcal{S}$ to const $_{D}$. This is a quasi-inverse for the functor taking $\left(\mathcal{F}_{A}, \mathcal{F}_{B}, U_{S}, U_{R}\right)$ to $\left(\mathcal{F}_{A}, U_{B}\right)$. Similarly, a transformation $\left(\mathcal{F}_{B}, U_{B}\right)$ from $\mathcal{S}_{2}$ to const $_{D}$ induces a transformation from $\mathcal{S}$ to $\operatorname{const}_{D}$. By the universal property of the colimit, this means that the diagrams $\mathcal{S}, \mathcal{S}_{1}$ and $\mathcal{S}_{2}$ have the same colimit.

Similarly,

Proposition 4.12. Let $\mathcal{S}$ be the functor $\mathcal{C}_{s} \rightarrow \mathfrak{G r}_{\text {prop }}$ corresponding to the strong shift equivalence<smiles>[R12][H]</smiles>

where $\mathcal{G}$ and $\mathcal{H}$ are groupoids and $\mathcal{X}_{R}$ and $\mathcal{X}_{S}$ are groupoid correspondences. Let $\mathcal{S}_{1}$ and $\mathcal{S}_{2}$ be the diagrams $(\mathbb{N},+) \rightarrow \mathcal{G}_{\text {prop }}$ generated by $\mathcal{X}_{R} \circ \mathcal{H} \mathcal{X}_{S}: \mathcal{G} \rightarrow \mathcal{G}$ and $\mathcal{X}_{S} \circ \mathcal{G} \mathcal{X}_{R}: \mathcal{H} \rightarrow \mathcal{H}$, respectively. Then

$$
\operatorname{colim}(\mathcal{S}) \cong \operatorname{colim}\left(\mathcal{S}_{1}\right) \cong \operatorname{colim}\left(\mathcal{S}_{2}\right)
$$

in $\mathfrak{C o r r}_{\mathrm{prop}}$, that is, these colimits are equivalent. 
4 Colimit equivalent diagrams 


\section{Bibliography}

[1] Suliman Albandik and Ralf Meyer, Product systems over Ore monoids, Doc. Math. 20 (2015), 1331-1402, available at http://www.math.uni-bielefeld.de/documenta/vol-20/38.html. MR 3452185

[2] Colimits in the correspondence bicategory, Münster J. Math. (2015), accepted. arXiv:1502.07771.

[3] William Arveson, Continuous analogues of Fock space, Mem. Amer. Math. Soc. 80 (1989), no. 409, iv+66, DOI 10.1090/memo/0409. MR 987590

[4] Teresa Bates, Applications of the gauge-invariant uniqueness theorem for graph algebras, Bull. Austral. Math. Soc. 66 (2002), no. 1, 57-67, DOI 10.1017/S0004972700020670. MR 1922607

[5] Teresa Bates and David Pask, Flow equivalence of graph algebras, Ergodic Theory Dynam. Systems 24 (2004), no. 2, 367-382, DOI 10.1017/S0143385703000348. MR 2054048

[6] Jean Bénabou, Introduction to bicategories, Reports of the Midwest Category Seminar, Springer, Berlin, 1967, pp. 1-77, DOI 10.1007/BFb0074299, (to appear in print)MR 0220789

[7] Nicolas Bourbaki, Topologie générale. Chapitres 1 à 4, Éléments de mathématique, Hermann, Paris, 1971. MR 0358652

[8] Mădălina Roxana Buneci, Groupoid categories, Perspectives in operator algebras and mathematical physics, Theta Ser. Adv. Math., vol. 8, Theta, Bucharest, 2008, pp. 27-40. MR 2433025

[9] Alcides Buss, Ruy Exel, and Ralf Meyer, Inverse semigroup actions as groupoid actions, Semigroup Forum 85 (2012), no. 2, 227-243, DOI 10.1007/s00233-012-9418-y. MR 2969047

[10] Alcides Buss and Ralf Meyer, Inverse semigroup actions on groupoids, Rocky Mountain J. Math. (2016), accepted. arXiv:1410.2051.

[11] Alcides Buss, Ralf Meyer, and Chenchang Zhu, A higher category approach to twisted actions on $\mathrm{C}^{*}$-algebras, Proc. Edinb. Math. Soc. (2) 56 (2013), no. 2, 387-426, DOI 10.1017/S0013091512000259. MR 3056650

[12] Alain Connes, Noncommutative geometry, Academic Press Inc., San Diego, CA, 1994. MR 1303779

[13] Joachim Cuntz and Wolfgang Krieger, A class of $C^{*}$-algebras and topological Markov chains, Invent. Math. 56 (1980), no. 3, 251-268, DOI 10.1007/BF01390048. MR 561974

[14] Valentin Deaconu, Continuous graphs and $C^{*}$-algebras, Operator theoretical methods (Timişoara, 1998), Theta Found., Bucharest, 2000, pp. 137-149. MR 1770320

[15] Douglas John Drinen and Nándor Sieben, $C^{*}$-equivalences of graphs, J. Operator Theory 45 (2001), no. 1, 209-229, available at http://www.theta.ro/jot/archive/2001-045-001/ 2001-045-001-011.html. MR 1823069

[16] Ruy Exel, Inverse semigroups and combinatorial $C^{*}$-algebras, Bull. Braz. Math. Soc. (N.S.) 39 (2008), no. 2, 191-313, DOI 10.1007/s00574-008-0080-7. MR 2419901 


\section{BIBLIOGRAPHY}

[17] Neal J. Fowler, Discrete product systems of Hilbert bimodules, Pacific J. Math. 204 (2002), no. 2, 335-375, DOI 10.2140/pjm.2002.204.335. MR 1907896

[18] Rohit Dilip Holkar, Topological construction of $\mathrm{C}^{*}$-correspondences for groupoid $\mathrm{C}^{*}$-algebras, Ph.D. Thesis, Georg-August-Universität Göttingen, 2014, http://hdl.handle.net/11858/ 00-1735-0000-0023-960F-3.

[19] Tsuyoshi Kajiwara, Claudia Pinzari, and Yasuo Watatani, Jones index theory for Hilbert $C^{*}$-bimodules and its equivalence with conjugation theory, J. Funct. Anal. 215 (2004), no. 1, 1-49, DOI 10.1016/j.jfa.2003.09.008. MR 2085108

[20] Takeshi Katsura, A class of $C^{*}$-algebras generalizing both graph algebras and homeomorphism $C^{*}$-algebras. I. Fundamental results, Trans. Amer. Math. Soc. 356 (2004), no. 11, 4287-4322, DOI 10.1090/S0002-9947-04-03636-0. MR 2067120

[21] Masoud Khalkhali, Basic noncommutative geometry, EMS Series of Lectures in Mathematics, European Mathematical Society (EMS), Zürich, 2009. MR 2567651

[22] Mahmood Khoshkam and Georges Skandalis, Regular representation of groupoid $C^{*}$-algebras and applications to inverse semigroups, J. Reine Angew. Math. 546 (2002), 47-72, DOI 10.1515/crll.2002.045. MR 1900993

[23] Alex Kumjian, David Pask, Iain Raeburn, and Jean Renault, Graphs, groupoids, and CuntzKrieger algebras, J. Funct. Anal. 144 (1997), no. 2, 505-541, DOI 10.1006/jfan.1996.3001. MR 1432596

[24] Alex Kumjian, David Pask, and Aidan Sims, Generalised morphisms of k-graphs: $k$-morphs, Trans. Amer. Math. Soc. 363 (2011), no. 5, 2599-2626, DOI 10.1090/S0002-9947-2010-051524-2. 2763728

[25] Tom Leinster, Basic Bicategories (1998), eprint. arXiv:math/9810017.

[26] Douglas Lind and Brian Marcus, An introduction to symbolic dynamics and coding, Cambridge University Press, Cambridge, 1995. MR 1369092

[27] Saunders MacLane, Categories for the working mathematician, Springer, New York, 1971. Graduate Texts in Mathematics, Vol. 5. MR 0354798

[28] Dmitry Matsnev and Pedro Resende, Étale groupoids as germ groupoids and their base extensions, Proc. Edinb. Math. Soc. (2) 53 (2010), no. 3, 765-785, DOI 10.1017/S001309150800076X. MR 2720249

[29] Kengo Matsumoto and Hiroki Matui, Continuous orbit equivalence of topological Markov shifts and Cuntz-Krieger algebras, Kyoto J. Math. 54 (2014), no. 4, 863-877, DOI 10.1215/215622612801849. MR 3276420

[30] J. P. May and J. Sigurdsson, Parametrized homotopy theory, Mathematical Surveys and Monographs, vol. 132, American Mathematical Society, Providence, RI, 2006. MR 2271789

[31] Ralf Meyer and Chenchang Zhu, Groupoids in categories with pretopology, Theory Appl. Categ. 30 (2015), 1906-1998, available at http://www.tac.mta.ca/tac/volumes/30/55/ 30-55abs.html.

[32] Paul S. Muhly, David Pask, and Mark Tomforde, Strong shift equivalence of $C^{*}$-correspondences, Israel J. Math. 167 (2008), 315-346, DOI 10.1007/s11856-008-1051-9. MR 2448028

[33] Paul S. Muhly and Mark Tomforde, Topological quivers, Internat. J. Math. 16 (2005), no. 7, 693-755, DOI 10.1142/S0129167X05003077. MR 2158956

[34] Paul S. Muhly, Jean N. Renault, and Dana P. Williams, Equivalence and isomorphism for groupoid $C^{*}$-algebras, J. Operator Theory 17 (1987), no. 1, 3-22, available at http: //www.theta.ro/jot/archive/1987-017-001/1987-017-001-001.html. MR 873460 
[35] Paul S. Muhly and Dana P. Williams, Renault's equivalence theorem for groupoid crossed products, NYJM Monographs, vol. 3, State University of New York University at Albany, Albany, NY, 2008. MR 2547343

[36] Volodymyr Nekrashevych, Self-similar groups, Mathematical Surveys and Monographs, vol. 117, American Mathematical Society, Providence, RI, 2005. MR 2162164

[37],$C^{*}$-algebras and self-similar groups, J. Reine Angew. Math. 630 (2009), 59-123, DOI 10.1515/CRELLE.2009.035. MR 2526786

[38] Alan L. T. Paterson, Groupoids, inverse semigroups, and their operator algebras, Progress in Mathematics, vol. 170, Birkhäuser Boston Inc., Boston, MA, 1999. MR 1724106

[39] Mihai V. Pimsner, A class of $C^{*}$-algebras generalizing both Cuntz-Krieger algebras and crossed products by $\mathbf{Z}$, Free probability theory (Waterloo, ON, 1995), Fields Inst. Commun., vol. 12, Amer. Math. Soc., Providence, RI, 1997, pp. 189-212. MR 1426840

[40] Jean Renault, A groupoid approach to $\mathrm{C}^{*}$-algebras, Lecture Notes in Mathematics, vol. 793, Springer, Berlin, 1980. MR 584266

[41] _ Représentation des produits croisés d'algèbres de groupoïdes, J. Operator Theory 18 (1987), no. 1, 67-97, available at http://www.theta.ro/jot/archive/1987-018-001/ 1987-018-001-005.html. MR 912813

[42] Marc A. Rieffel, Morita equivalence for $C^{*}$-algebras and $W^{*}$-algebras, J. Pure Appl. Algebra 5 (1974), 51-96, DOI 10.1016/0022-4049(74)90003-6. MR 0367670

[43] Nándor Sieben, $C^{*}$-crossed products by partial actions and actions of inverse semigroups, J. Austral. Math. Soc. Ser. A 63 (1997), no. 1, 32-46, DOI 10.1017/S1446788700000306. MR 1456588

[44] Mark Tomforde, Strong shift equivalence in the $C^{*}$-algebraic setting: graphs and $C^{*}$-correspondences, Operator theory, operator algebras, and applications, Contemp. Math., vol. 414, Amer. Math. Soc., Providence, RI, 2006, pp. 221-230, DOI 10.1090/conm/414, (to appear in print). MR 2277213

[45] Jean-Louis Tu, Non-Hausdorff groupoids, proper actions and K-theory, Doc. Math. 9 (2004), 565-597, available at http://www.math. uni-bielefeld.de/documenta/vol-09/26.htmlMR 2117427

[46] Trent Yeend, Groupoid models for the $C^{*}$-algebras of topological higher-rank graphs, J. Operator Theory 57 (2007), no. 1, 95-120, available at http://www.theta.ro/jot/archive/ 2007-057-001/2007-057-001-005.html. MR 2301938 


\section{Curriculum vitae of Suliman Albandik}

\section{Personal data}

Name, Title

Suliman Albandik

Sex

Male

Address

Georg-August-Universität Göttingen

Mathematisches Institut

Bunsenstraße 3-5

37073 Göttingen

Phone

+49(0) 551397751

Email

albandik@uni-goettingen.de

Website

www.uni-math.gwdg.de/suliman/

Position

Postdoc stipend from the RTG 1493

Children

Three, born 03.07.2009, 23.11.2013 and 15.04.2015.

\section{Academic education including academic degrees}

09/2003-07/2006 B.Sc. Mathematical Analysis, Tishreen Univ., Latakia, Syria

10/2009-10/2010 M.Sc. student in mathematics, Universität Göttingen

\section{Scientific graduation}

10/2010-08/2015 Doctoral studies in mathematics, Universität Göttingen oral exam: 10.08.2015, supervisor: Ralf Meyer

\section{Language skills}

English (fluent), German (fluent), Arabic (native speaker)

\section{Awards and scholarships}

2003, 2004, 2005 Al-Bassel award for excellent high school performance

2006

$2008-2014$

\section{Employment}

01/2015-08/2015 doctoral stipend 


\section{Other activities}

04/2014-08/2015 Speaker of doctoral students in the School of Mathematical Sciences at Universität Göttingen

10/2008-06/2009 Intensive German language course at Tischreen Univ., Latakia, Syria

\section{Thesis}

Doctorate A Colimit construction for groupoids (advised by Ralf Meyer)

\section{Two publications}

\section{a) Reviewed}

1. S.Albandik, R. Meyer. Product systems over Ore monoids. Doc. Math. 20 (2015), 1331-1402.

Available at http://www.math.uni-bielefeld.de/documenta/vol-20/38.html

2. S.Albandik, R. Meyer. Colimits in the correspondence bicategory. Münster J. Math., accepted.

Available at http://wwwmath1.uni-muenster.de/mjm/acc/Albandik.pdf

\section{Work in progress}

1. S.Albandik, Colimit equivalence for étale groupoids and $\mathrm{C}^{*}$-algebras.

2. S.Albandik, R. Meyer. A limit construction for étale topological groupoids

3. S.Albandik, R. Holkar. Proper topological correspondences.

\section{Presentations}

1. A short introduction to the theory of $\mathrm{C}^{*}$-algebras, Utah Valley University, Orem, Utah (United States) May 23, 2012

2. KMS states for $\mathrm{C}^{*}$-algebras associated with self-similar groups, Utah Valley University Orem, Utah (United States) May 25, 2012

3. KMS states for $\mathrm{C}^{*}$-algebras associated with self-similar groups, RTG 1493 meeting, Goslar (Germany) October 8, 2012

4. Crossed Products and Cuntz-Pimsner algebras as colimits, poster session, Workshop Groups, Dynamical Systems and $\mathrm{C}^{*}$-Algebras, Münster (Germany) August 20-24, 2013. 
5. Crossed Products and Cuntz-Pimsner algebras as colimits, School and workshop: Topics in Operator Algebras and Applications, Madrid (Spain) September 2nd-6th, 2013.

6. Crossed Products and Cuntz-Pimsner algebras as colimits, K-Theory and Index Theory, Metz, France, June 2-6, 2014.

\section{Research visits}

$05 / 2012$

$07 / 2015$

Visiting Machiel van Frankenhuijsen, Utah Valley University.

Visiting Aidan Sims during his stay in Münster.

\section{Events}

1. School on Higher Index Theory and Differential K-theory, Göttingen, 18-22.10.2010

2. School on Geodesic Flow, Negative Curvature and Isomorphism Conjectures, Göttingen, 21.-24.06.2011

3. Conference on K-Theory, $\mathrm{C}^{*}$-Algebras and Index Theory, Göttingen, 1.-5.11.2010

4. School on Metric Measure Spaces: Geometric and Analytic Aspects, Montréal (Kanada), 27.06.-08.07.2011

5. School on Noncommutative Geometry: Multiple Connections. Columbus, $\mathrm{OH}$ (United States), May 7-18, 2012

6. Conference on Quantum Geometry, Anogia (Greece), 6-10 August 2012

\section{Teaching}

Winter

$2011 / 2012$

$09 / 2006-08 / 2009$
Noncommutative geometry III by Ralf Meyer: Teaching assistant.

Teaching Assistant in the mathematics institute, Tishreen University.

Teaching Assistant in the statistical mathematics institute. 